\section{KEYNOTE LECTURES:}

\section{Keynote lecture I:}

\section{HOLLAND: RCT-LAND (KLI)}

J. Jeekel

Erasmus Medical Center Rotterdam, Rotterdam, The Netherlands

Evidence based medicine is largely based upon results of Randomised Controlled Trials(RCT). RCT's are difficult to perform. It requires a number of distinctive characteristics, a scientific and critical mind, an environment which allows a critical approach to existing treatments, the ability to cooperate and communicate, a perfect organisation to perform joined studies and the knowledge and ability to conceive a new study,write the protocol ,perform the study,collect data , interpret data and write articles in a scientific fashion which lead to publication in international journals. These characteristics should not only be present in one person or center, but have a general backpround in order to create a general support for the wish to critically evaluate healthcare and initiate studies to improve patient treatment.

All these characteristics are not present in every country. It seems that the necessary elements are sufficiently present in the Netherlands. Holland appears to be one of the most productive countries in terms of output of surgical papers. Furthermore the number of RCT's performed in the Netherlands is extremely high. Most of the surgical studies are investigator driven instead of industry driven. Of all RCT.s only a small percentage is derived from experimental studies performed in the own laboratory. It would be of great value to find ways to create the critical scientific environment in countries that are not yet very productive in clinical trials. In order to do so, we should know the common denominators, the necessary elements that lie at the basis of such scientific environment. Experimental research will help in creating a critical mind and may stimulate the conception of good clinical studies.

\section{Keynote lecture II:}

\section{POSSIBLE APPLICATIONS OF NANOTECHNOLOGY IN MEDICINE (KLII)}

\section{D.H.A. Blank}

Scientific Director MESA+ Institute for Nanotechnology, Enschede, The Netherlands

Nanomedicine is the application of nanotechnology in medicine. It deals with nanoscale (1 to $100 \mathrm{~nm}$ ) biomolecular, inorganic or hybrid structures, devices, and technical or biological systems with novel properties which have potential applications in the prevention, diagnosis, and treatment of disease and genetic deficiencies. With rapid progress in molecular biology and medicine as well as experimental technology, the molecular scale is now becoming accessible. Examples are the use of nanoparticles as labels in intracellular imaging and molecular diagnostics or the use of nanoscale containers such as viral shells as precisely targeted drug delivery vehicles. Single cancer cells could be eliminated in the earliest state of cancer. A next step using more complex nanodevices would be molecular diagnostics combined with therapy. Implants with nanoscale functional parts (such as electrodes) could be used for ultrasensitive biochemical analysis in the body, local and targeted drug delivery, and wireless reporting of progress. Mass-produced and inexpensive in vitro biosensors would enable family doctors or patients themselves to routinely detect extremely small concentrations of DNA, proteins, hormones, drugs, metabolites or pathogens in body fluids, or of gases in exhaled air. Nanomedicine clearly is a highly interdisciplinary endeavor, including physical, chemical and biological sciences, engineering sciences and clinical medicine. An eventual success in nanomedicine will be inseparably connected to a quantitative understanding of the physical/chemical properties and engineering principles of the molecular building blocks and nanomachines in cells. A crucial part of a nanomedicine initiative will therefore be strong support for basic science elucidating the microscopic dynamics and functional properties of biomolecular nanomachines and assemblies in cells. In this presentation, starting with the explanation of what is nanotechnology, I will go in more detail on the possibilities of nanotechnology, what are the limitations, what is the future and what the possible impact on society.

\section{Keynote Lecture III:}

\section{NOVEL INSIGHTS IN POST-ISCHEMIC NEOVASCULARIZATION (KLIII)}

\author{
J.S. Silvestre \\ Cardiovascular Research Center Inserm U689 Lariboisiere, France
}

In embryogenesis, the vascular system develops from vasculogenesis in which endothelial cell precursors differentiate into endothelial cells to form primitive capillary network. The subsequent growth, expansion and remodeling of these primitive vessels into a mature capillary network is referred to as angiogenesis. The nascent vessels are then stabilized by recruiting mural cells and generating extracellular matrix, a process termed arteriogenesis. However, a large body of evidence underscores that neovascularization also proceeds in adult during pathological conditions such as tumor growth or ischemic diseases. The three principal processes, vasculogenesis, angiogenesis and arteriogenesis characterize tissue repair and remodeling occuring in acute and chronic ischemic vascular diseases, and represent the final targets of therapeutic angiogenesis aimed at providing an alternative treatment strategy for patients with lower limb ischemia and coronary artery diseases. Numerous strategies of therapeutic angiogenesis based on administration of growth factors, progenitor cells from bone marrow and non bone marrow origins, or extracellular matrix protein have been shown to promote revascularization in animal models of cardiac or hindlimb ischemia. Some of them are of particular interest and are now tested at the clinical levels in patients with acute myocardial infarction or peripheral artery diseases. However, molecular and cellular mechanisms involved in post-ischemic revascularization need to be further identified and defined. In particular, strategies to increase homing, survival and therapeutic potential of progenitor cells need to be developped to improve therapeutic effect and counteract progenitor cells dysfunction in aged patients with cardiovascular risk factors.

\section{Keynote Lecture IV:}

\section{TRANSLATIONAL RESEARCH:}

"FROM THE LAB TO THE CLINIC AND BACK TO THE LAB" (KLIV)

\section{A.M.M.Eggermont \\ ErasmusMC-Daniel Den Hoed Cancer Center, Rotterdam, The Netherlands}

The landscape for clinical research is profoundly different today from that only one ecade ago. Basic scientific discoveries follow an exponential curve and are implemented in clinical research and in clinical practice at a much greater speed than ever before. This situation is demanding for all those involved in research and health care. It requires adaptation, creates new roles and opportunities for all. This is particularly poignent in the field of implementing translational research as a mandatory component and driving force in clinical research in oncology. The surgeon should be a key-player in this process. Traditionally translational research has been broadly defined as "bringing basic scientific discoveries to clinical practice", typically "from bench to bed, from laboratory to the clinic". In the days of genomics, proteomics and metabolomics, translational research means increasingly "from the clinic back to the lab". With todays technologies we move from descriptive visual observations on patients tissues to the molecular and biometrically analised discovery of fundamentally new knowledge and insights. This is examplified for instance by recent developments where gene profiling has lead to a new classification of breast cancer ${ }^{1}$ and recently to further subclassifications, to prognostic signatures that are now commercially available and in clinical practice, and to predictive signatures determined in neoadjuvant treatment protocols for treatment outcome ${ }^{2}$. Recently a general "invasiveness gene signature or IGS" was descovered in breast cancer that has not only a strong predictive signature for breast cancer, but also for medullo blastoma, lung cancer, and prostate cancer ${ }^{3}$. Neoadjuvant treatment protocols provide important opportunities to identify predictive signatures that will establish new criteria for subsequent surgery, radiotherapy and systemic treatment strategies. Locally advanced rectal cancers, oesophageal, gastric, and pancreatic cancers are all examples that spring to mind. Obviously translational research driven trials could help to identify patients that will, may, may not, and certainly will not benefit from surgery. Similarly neoadjuvant therapy trials have identified patients with liver metastases that will and will not benefit from liver resection. Similar developments are ongoing in many 
other cancers, and this process will greatly enhance our understanding of the biology of these tumours and will lead to new treatment strategies. It is exactly in this process that surgeons can play a crucial and invaluable role. It is the surgeon who can guarantee high quality tissue samples, provide important peritumoral tissue ${ }^{4}$ and draining lymphnodes as well as distant metastases. Since the quality of tissue depends on tissue handling and proper logistics it is clear that a true engagement and key role for the surgeon in translational research programs will be quite important. It requires however that academic surgeons have a profound interest in biology and research and that they are willing to tackle the regulatory hurdles that complicate the conduct of clinical research today. It also means that academic surgeons will find it difficult to keep up to speed with any of the current developments without formal training in molecular biology and "omics". This can be acquired to some degree by special courses, but formal research period of at least two years in the laboratory is the best way to achieve a deeper understanding of the value and opportunities, of the variability of tissue quality and new assay methodologies, and thus create awareness and appreciation of both the opportunities and the limits of new technologies and bioinformatics. The surgeon should be critically involved in the implementation of standard operating procedures (SOPs) for tissue sampling, handling, storage as this is crucial to be able to trust the results of the subsequent assays performed on these tissues. The surgeon should be not only aware of this, but in the lead of organising and supervising logistics, as these start in the operation theatre. In concert with excellence in bio-banking SOPs for tumor tissues, the storage of blood, plasma and serum samples should be organised with the same precision. Protein functionality is "written in blood". Mapping tumor cell protein networks in vivo will be critical for realizing the promise of patient-tailored molecular therapy. Until recently, the field lacked the technology for molecular profiling at the genomic and proteomic level. Emerging proteomic technology, used concomitantly with genomic analysis, promises to meet this need and bring to reality the clinical adoption of molecular stratification. ${ }^{5}$ Academic surgeons have a choice to make. Either go all the way and secure their position as key participants in research that seems more exciting than ever, or become just a provider and loose out on being part of a revolution in medecine. The rewards will be just as spectacular and practice-changing as the discovery of the Heliobacter pylori changed the field of gastric surgery. Patients that enroll into trials expect that serious biologic questions will be addressed that will move the field forward. Failure to address such questions as primary or secondary endpoints in clinical research will be viewed a scientific criminal neglect. Never was there a better time for surgeons to study biology.

1 Sorlie T, Perou CM, Tibshirani R, et al. Gene expression patterns of breast carcinomas distinguish tumor subclasses with clinical implications. Proc Natl Acad Sci U S A. 2001 Sep 11;98(19):10869-74.

2 Iwao-Koizumi K, Matoba R, Ueno N, et al K. Prediction of docetaxel response in human breast cancer by gene expression profiling. J Clin Oncol. 2005 Jan 20;23(3):422-31

3 Liu R, Wang X, Chen GY, Dalerba P,et al. The prognostic role of a gene signature from tumorigenic breast-cancer cells. $\mathrm{N}$ Engl J Med. 2007 Jan 18;356(3):217-26.

4 Schneider S, Park DJ, Yang D, et al. Gene expression in tumoradjacent normal tissue is associated with recurrence in patients with rectal cancer treated with adjuvant chemoradiation. Pharmacogenet Genomics. 2006 Aug;16(8):555-63.

5 Petricoin EF 3rd, Bichsel VE, Calvert VS, et al. Mapping molecular networks using proteomics: a vision for patient-tailored combination therapy. J Clin Oncol. 2005 May 20;23(15):3614-21. Review.

\section{ORAL PRESENTATIONS:}

\section{O001}

UPREGULATION OF ENOS PREVENTS ENDOTHELIAL DYSFUNCTION AND INCREASED MICROVASCULAR THROMBUS FORMATION DURING TREATMENT WITH DARBEPOETIN-ALPHA

N. Lindenblatt ${ }^{1}$, M.D. Menger ${ }^{2}$, E. Klar², B. Vollmar²

1 University of Rostock, Rostock, Germany

2 Institute for Clinical \& Exp. Surgery Homburg/Saar, Germany

Introduction: Erythropoietin-treatment is generally assumed to be associated with an increased risk for thrombosis due to an elevated hematocrit. However, previous in vitro and in vivo studies of our group revealed a reduction of platelet reactivity and no significant effect on microvascular thrombus formation during treatment with darbepoetin-al- pha (DPO), an erythropoietin-derivative with a 3-fold longer half life. Aim of the study was therefore to investigate the effect of DPO application on endothelial function and to further characterize the role of eNOS in this context.

Materials and methods: C57BL/6J mice were treated with DPO for 4 weeks (1, 10 und $25 \mu \mathrm{g} / \mathrm{kg}$ bw sc, $1 \mathrm{x} /$ week). In microvessels of mouse cremaster muscle preparations ferric chloride-induced thrombus formation was analyzed using intravital fluorescence microscopy. Additionally systemic circulation of the endothelial activation markers SP-selectin, sE-selectin, sICAM und sVCAM was determined by ELISA. Endothelia expression of eNOS within the cremaster muscle tissue was evaluated using immunohistochemistry and RT-PCR. To clarify the meaning of eNOS in vivo eNOS knock-out mice (B6.129P2/Nos3; eNOS -/-) were pretreated with DPO $(10 \mu \mathrm{g} / \mathrm{kg}$ bw sc, $1 \mathrm{x} /$ week $)$ and microvascular thrombus formation within the cremaster muscle was analysed. Results: Chronic treatment of eNOS-competent wild-type mice with 10 und $25 \mu \mathrm{g} / \mathrm{kg}$ DPO caused a significant increase in hematocrit ( $68 \pm 4 \%$ vs. control: $47 \pm 0 \% ; p<0.05$ ), haemoglobin concentration $(19.0 \pm 1.6 \mathrm{~g} / \mathrm{dl}$ vs. $13.6 \pm 0.1 \mathrm{~g} / \mathrm{dl} ; \mathrm{p}<0.05)$ and reticulocyte count $(6.5 \pm 0.3 \%$ vs. $2.7 \pm 0.2 \% ; p<0.05)$, when compared to controls. In addition ELISA revealed a significant reduction of circulating $\mathrm{SP}$-selectin and SICAM-1 in DPO-treated wild-type mice. Immunhistochemistry and RT-PCR analysis showed a significant $(p<0.05)$ increase of eNOS expression following chronic DPO-treatment. Concurrently no significant differences in kinetics of arteriolar and venular microvascular thrombus formation were observed between DPO-treated wild type animals and untreated control animals. In contrast to this untreated eNOS -/- mice already revealed a slight acceleration in microvascular thrombus formation. Additional chronic application of DPO resulted in a significant reduction of times until complete vessel occlusion when compared to control eNOS -/- mice.

Conclusion: Chronic application of DPO causes a reduction of platelet and endothelial activation. This reduction is most likely mediated via an upregulation of eNOS, which can be interpretated as a mechanism compensating potential hematocrit-associated prothrombogenicity generated by DPO. Thus, it is shown, that chronic treatment with DPO is not necessarily linked to an increased risk for microvascular thrombus formation, as long as an adequate eNOS counterbalance of the endothelium in terms of anti-thrombogenicity is guaranteed.

\section{$\mathrm{O} 002$}

DEVELOPMENT OF NON-ANASTOMIC BILIARY STRICTURES AFTER HUMAN LIVER TRANSPLANTATION IS PRECEDED BY ABNORMAL BILE COMPOSITION IN THE EARLY POSTOPERATIVE PERIOD

\section{C.I. Buis, E. Geuken, D.S. Visser, H.J. Verkade, M.J.H. Slooff, R.J. Porte} UMCG, Groningen, Groningen, The Netherlands

Introduction: Biliary complications are reported in 10 to $30 \%$ of the patients after orthotopic liver transplantation (OLT), representing a major cause of morbidity and mortality. Non-anastomic biliary strictures (NAS) are considered to be the most troublesome one, leading to recurrent jaundice, cholangitis and potentially graft failure. The pathogenesis of NAS remains largely unknown.

Aim: To determine the impact of changes in bile composition and gene expression of hepatobiliary transporters on the development of NAS after OLT.

Patients and methods: In a prospective study, 105 consecutive adult liver transplant recipients were studied. Three liver needle biopsies were taken at the end of cold storage, $3 \mathrm{hr}$ after reperfusion and one week after OLT. Bile samples were collected daily from a biliary drain until 8 days after transplantation and bile flow was measured daily. To maintain the enterohepatic circulation, bile was readministered to the patient via a feeding jejunostomy. Biliary concentrations of bile acids, cholesterol and phospholipids were measured using biochemical and enzymatic methods. Expression of the hepatic transporter proteins for bile acids (NTCP and BSEP), phospilipds (MDR3) and bilirubine and GSH (MRP2) were quantified by measurement of mRNA using quantitative RT-PCR. Results: In this series of 105 patients, 14 patients developed NAS at a median time of 4 months (range 1-27) after OLT. These patients were compared with 61 patients who did not have any biliary complication or graft rejection (control group). There were no significant differences between the two groups with respect to patient or donor characteristics and serum liver enzymes during the first week after OLT. Also daily bile flow was not different between the two groups. However, biliary bile 
acid, phospholipid and cholesterol secretion during the first week after OLT was significantly lower in the patients who ultimately developed NAS, compared to controls. In addition, gene expression of the bile transporters NTCP, BSEP and MRP2 was significantly lower after graft reperfusion in donor livers which ultimately developed NAS.

Conclusion: Although the median time interval to diagnose NAS is 4 months after OLT and patients who develop NAS are clinically indiscernible during the first week after transplantation from patients who do not develop NAS, we found pronounced differences in bile composition during the very early postoperative period in these two groups of patients. In addition, the hepatic expression of bile transporters is markedly decreased after graft reperfusion in patients who will ultimately develop ITBL. These findings suggest that early defects in bile formation may play a role in the pathogenesis of NAS.

\section{O003}

PORCINE KIDNEY TRANSPLANTATION AFTER MACHINE PRESERVATION USING THE GRONINGEN HYPOTHERMIC ORGAN PERFUSION SYSTEM

M.H.J. Maathuis, $S^{1}$. Manekeller ${ }^{2}$, A. van der Plaats ${ }^{3}$,

H.G.D. Leuvenink', N. 't Hart', A.B. Lier', G. Rakhorst', R.J. Ploeg1',

T. Minor ${ }^{2}$

1 University Medical Center Groningen, Groningen, The Netherlands

2 Surgical Research Division, Bonn, Germany

3 Organ-Assist B.V., Groningen, Groningen, The Netherlands

Introduction: As many risk factors for transplantation outcome depend on the donor, optimization of organ viability is a key factor to success. One way to maintain donor organ viability is hypothermic machine perfusion (HMP). To utilize the full potential of HMP we developed a portable hypothermic organ perfusion system. The aim of this study was to evaluate this system in porcine kidney transplantation comparing graft function and ischemia reperfusion injury after HMP to static cold storage (CS). Furthermore, the optimal perfusion pressure during HMP was determined by using two different perfusion strategies

Materials and methods: Donor kidneys were retrieved from female landrace pigs $( \pm 30 \mathrm{~kg})$. After kidney retrieval, grafts were preserved at $4^{\circ} \mathrm{C}$ with either UW-CS ( $\left.n=5\right)$, HMP with UW-MP using $30 / 20 \mathrm{mmHg}$ $(n=5)$ or HMP with UW-MP using $60 / 40 \mathrm{mmHg}(n=5)$. Following preservation kidneys were auto-transplanted with removal of the contralateral kidney. Daily venous blood samples were taken to evaluate kidney function by means of serum creatinine. Cortical microcirculation after reperfusion was assessed by Laser Doppler flowmetry. Urinary biomarkers were used to quantify the following components of ischemia reperfusion injury: oxidative stress (TBARS), proximal tubule injury (enzyme release AAP and NAG ) and proteinuria. RT-PCR analysis of vascular injury markers (E-selectin, P-selectin, Von Willebrand factor (VWF) and monocyte chemotactic protein (MCP-1) was used to detect HMP related vascular damage.

Results: Peak serum creatinine as well as time to peak serum creatinine were significantly lower in both HMP groups. CS grafts showed a peak serum creatinine of $940 \pm 90 \mu \mathrm{mol} / \mathrm{l}$ on post operative day 3.4 \pm 0.89 . In contrast, animals that received HMP $30 / 20$ kidneys had a significantly lower peak creatinine of $463 \pm 127 \mu \mathrm{mol} / \mathrm{l}$ on postoperative day $1.8 \pm 1.1(p<0.05)$. Surviving pigs in the HMP 60/40 group showed similar results to HMP $30 / 20$, however, two animals died with a thrombosed graft. Evaluation of cortical microcirculation showed a higher flow index in HMP $30 / 20 \mathrm{mmHg}$ compared to CS kidney grafts (1.3 vs. 0.9, $(p<0.05))$. Analysis of biomarkers revealed less oxidative stress, less proximal tubule damage and less proteinuria in HMP groups compared to CS $(p<0.05)$. Vascular damage markers VWF and MCP-1 were elevated in HMP 60/40 kidneys compared to HMP $30 / 20$ and CS $(p<0.05)$. Conclusion: We conclude that using the Groningen machine perfusion system in porcine kidney transplantation results in better early graft function, improved cortical microcirculation and reduction of ischemia reperfusion injury compared to CS. Pulsatile perfusion pressures are, however, critically important. HMP at $30 / 20 \mathrm{mmHg}$ resulted in the best post transplant results in this experiment, while $60 / 40 \mathrm{mmHg}$ caused vascular damage due to shear stress.

\section{O004}

\section{THE EFFECTS OF PROBIOTICS IN THE PREVENTION OF LUNG INJURY IN ACUTE NECROTIZING PANCREATITIS}

K. Melike ${ }^{1}$, O. Yksel2 , N. Akyrek ${ }^{3}$, T. Sahin ${ }^{3}$, L. Memis ${ }^{4}$, N. Akyrek $^{4}$, H. Pasaoglu ${ }^{5}$, E. Ofluoglu ${ }^{5}$, K. Aglar ${ }^{6}, \mathrm{H}$. Bostanci $^{3}$
1 Gazi University Medical School, Ankara, Turkey

2 Department of surgery, Ankara, Turkey

3 Department of surgery, Ankara, Turkey

4 Department of pathology, Ankara, Turkey

5 Department of biochemistry, Ankara, Turkey

6 Department of microbiology, Ankara, Turkey

Background and aim: Pancreatic necrosis and infection are strong determinants of organ failure and poor outcome in severe acute pancreatitis. Acute necrotizing pancreatitis (ANP) results in serious pulmonary complications in $>50 \%$ of patients. Probiotics play an important role in the prevention of lung injury both through reducing the bacterial translocation and via regulating the cytokine liberation. In this study we aimed to investigate the effects of probiotics in reducing lung injury through decreasing bacterial translocation and systemic inflammatory response in an experimental model of ANP.

Materials and methods: ANP was induced by an intravenous infusion of cerulein $5 \mu \mathrm{g} / \mathrm{kg} / \mathrm{hour}$ over six ours superimposed on a standard infusion of $1.2 \mathrm{ml} / \mathrm{kg}$ glycodeoxycholic acid $10 \mathrm{mmol} / \mathrm{L}$ into biliary pancreatic duct for 10 minutes at $30 \mathrm{mmHg}$. Saccharomyces Boulardii (Reflor ${ }^{\mathrm{R}}$ Biocodex, $25 \mathrm{mg} / \mathrm{day}$ ) was used as the probiotic agent. Rats were divided into three groups: In the first group the rats were given only saline (at 6th and 24th hours following the initiation of the experiment) via gavage (control group, $n=10$ ), in the second group the animals were given saline (at 6th and 24th hours) via gavage following the induction of ANP (ANP+saline, $n=10$ ) and the third group were given probiotic (at 6th and 24th hours) agent via gavage following the induction of ANP $(A N P+$ probiotic, $n=10)$. Rats were sacrificed at 48 th hour and tissue and blood samples were collected. Research parameters are amylase, lactate dehydrogenase (LDH), IL-6, secretory phospholipase A2 (sPLA2), histopathological analysis of pancreatic and pulmonary tissues and microbiological assessment of mesenteric lymph nodes, pancreas and lung tissues.

Results: Serum levels of amylase, LDH, IL-6, sPLA2 were significantly higher in the ANP+saline group when compared to the ANP+probiotic and control groups $(p<0.05)$. Histopathologically there were reduced pancreatic edema, inflammation and vacuolization together with reduced lung edema and polymorphonuclear cell infiltration following probiotic administration. When the ANP+saline and ANP+probiotic groups were compared interms of microbiological assessment; bacterial colonization was significantly lower in the ANP+probiotic group $(\mathrm{P}<0.05)$.

Conclusion: Supplementation of probiotic agent Saccharomyces Boulardii attenuates proinflammatory cytokine levels, reduces bacterial translocation and decreases systemic inflammatory response which may be the reason of the reduced pancreatitis associated lung injury.

\section{O005}

PRETREATMENT WITH RECOMBINANT HUMAN ERYTHROPOIETIN PROTECTS CRITICALLY PERFUSED FLAP TISSUE FROM CHRONIC ISCHEMIA

F. Rezaeian ${ }^{1}$, R. Wettstein ${ }^{1}$, M. Amon², R. Schramm², B. Pittet ${ }^{1}$, M.D. Menger ${ }^{2}$, Y. Harder $^{1}$

1 University Hospitals of Geneva, Geneva, Switzerland

2 Institute for Experimental Surgery, Homburg/Saar, Germany

Introduction: The development for non-surgical preventive strategies that limit ischemic tissue damage of critically perfused flap tissue is a major research goal. In chronic ischemia of the brain, heart and kidney, erythropoietin (rhEPO), the primary regulator of erythropoiesis, has been demonstrated to exert cytoprotectant properties. Such beneficial effects however, are not known in flaps yet. Therefore, the aim of the present study was to analyze the effect of rhEPO on ischemia-induced microcirculatory dysfunction in critically perfused musculocutaneous flap tissue.

Materials and methods: 3 groups of 7 animals each were treated with 3 repetitive doses of $500 \mathrm{IU} / \mathrm{kg}$ bw rhEPO i.p. In group one, rhEPO was administrated before (PRE: $48 \mathrm{~h}, 24 \mathrm{~h}, 30 \mathrm{~min}$ ) surgery. In group 2, rhEPO was administered before and after (PRE\&POST: 30', surgery, 30', 24h) surgery. In group 3, rhEPO was administered after (POST: surgery, 30', $24 \mathrm{~h}, 48 \mathrm{~h}$ ) surgery. In a fourth group, animals received saline only for controls (CON: $n=7)$. Chronic ischemia was induced by elevating a randomly perfused flap in the back of C57BL/6-mice subsequently fixed into a dorsal skinfold chamber. Repetitive in vivo fluorescence microscopic analyses were performed over a 10-day observation period assessing 
nutritive microcirculation, leukocyte-endothelial cell interaction, apoptotic cell death and tissue necrosis. Rheology including hematocrit was repetively measured in separate animals $(n=8)$.

Results: The hematocrit did not show any increase in any treatment group (rhEPO: $28.2 \pm 2.1 \%$ vs CON: $27.0 \pm 3.9 \%$; d6). In untreated flaps, chronic ischemia resulted in a flap necrosis of $48 \pm 2 \%$ (CON). Pretreatment with rhEPO drastically reduced flap necrosis (PRE: $26 \pm 3 \%$ : $p<0.05$ vs CON: PRE\&POST: $20 \pm 3 \%$ : $p<0.05$ vs. CON). In contrast, administration of rhEPO after elevation of the flap did not affect the normal course of flap necrosis (POST: $45 \pm 5 \%$ ). Reduction of flap necrosis in groups 1 and 2 was associated with arteriolar dilation, increased arteriolar blood flow, and hence improved capillary perfusion as well as decreased apoptotic cell death $(p<0.05)$. Furthermore, rhEPO diminished ischemiainduced leukocytic inflammation by reducing the number of rolling and firmly adhering leukocytes in postcapillary venules $(p<0.05)$. Of interest, rhEPO, in particular if applied before induction of chronic ischemia, additionally induced angiogenic budding and sprouting resulting in formation of new capillary network visible at day 5 .

Conclusion: Systemic rhEPO administrated before induction of chronic ischemia effectively attenuates from flap necrosis by maintaining nutritive microcirculation, reducing leukocytic inflammation and inducing new vessel formation. In contrast, treatment after flap elevation does not prevent ischemic tissue damage. Thus, pretreatment using rhEPO may represent a promising strategy to reduce ischemia-related complications potentially endangered clinical flap surgery without affecting hematocrit.

\section{O006}

OVEREXPRESSION OF HUMAN ENDOTHELIAL NITRIC OXIDE SYNTHASE RESTORES POST-NATAL NEOVASCULARIZATION IN DIABETIC MICE

B.M.E. Mees ${ }^{1}$, L. Waeckel' ${ }^{2}$, T. Ebrahimian'2, D. You ${ }^{2}$, R. Van Haperen ${ }^{3}$, J. Vilar', D. Tempel ${ }^{3}$, B. Levy², R. De Crom ${ }^{3}$, J.S. Silvestre ${ }^{2}$

1 Erasmus University Medical Center Rotterdam, Rotterdam,

The Netherlands

2 Inserm U689, Paris, France

3 Erasmus University Medical Center Rotterdam, Rotterdam,

The Netherlands

Introduction: Cardiovascular disease is a common complication of diabetes with often a decreased post-natal vasculogenesis. In diabetic mice post-ischemic neovascularization is impaired. Furthermore, diabetes results in the abrogation of bone-marrow mononuclear cells (BM-MNC) pro-angiogenic potential. Overexpression of eNOS has been shown to promote vessel growth in the setting of ischemia. We therefore hypothesized that elevation of eNOS expression can restore the impaired post-natal vasculogenesis and neovascularization in diabetic mice.

Methods: Hind limb ischemia was induced in the mice by right femoral artery ligation. Two weeks after ligation we evaluated tissue perfusion of the foot by Laser Doppler Imaging (LDI), vessel density in the hind limb by micro-angiography and capillary density by histology.

Results: Tissue perfusion and vessel density were 1.5 -fold increased in transgenic mice overexpressing human eNOS (eNOStg) as compared to wild type C57BI6 (WT) mice $(\mathrm{P}<0.001, \mathrm{n}=10)$. Furthermore, intravenous transplantation of $1 \times 106$ WT BM-MNC or eNOStg BM-MNC in WT recipient mice $24 \mathrm{hrs}$ after ligation both caused a 1.5-fold increase in tissue perfusion and vessel density compared to injection of PBS $(P<0.001, n=10)$.

In a second set of experiments we induced severe hyperglycemia in eNOStg and WT mice by injecting streptozotocin. Both tissue perfusion and vessel density were 1.5 -fold increased in eNOStg ${ }^{*} \mathrm{DIAB}$ mice as compared to $W^{*}$ DIAB mice $(P<0.01, n=10)$. In addition, intravenous transplantation of eNOStg*DIAB BM-MNC in WT and WT*DIAB recipients caused a 1.4- to 1.7 -fold increase in tissue perfusion and vessel density compared to PBS $(P<0.01, n=10)$, while transplantation of WT*DIAB BM-MNC had no positive effects on tissue perfusion and vessel density compared to PBS. Finally, eNOS overexpression did not affect BM-MNC apoptosis but increased their ability to proliferate and differentiate in vitro into endothelial progenitor cells.

Conclusion: The above findings demonstrate that eNOS overexpression in the endothelium improves post-ischemic neovascularization in both physiological (WT) as diabetic settings. Furthermore, eNOS overexpression in the bone marrow restores the impaired pro-angioge- nic potential of diabetic bone marrow cells, when transplanted in both healthy (WT) and diabetic recipients. Therefore, overexpression of eNOS could play a vital part in the development of therapeutic angiogenesis for cardiovascular disease caused by diabetes.

\section{O007}

\section{ADENOVIRUS-MEDIATED VEGF GENE THERAPY ENHANCES VE-} NOUS THROMBUS RECANALISATION AND RESOLUTION

\section{B. Modarai ${ }^{1}$, J. Humphries ${ }^{1}$, J. Gossage ${ }^{1}$, K. Burnand ${ }^{1}$, A. Afuwape ${ }^{2}$,}

E. Paleolog' ${ }^{2}$, A. Smith 1

1 King's College, London, United Kingdom

2 Imperial College, London, United Kingdom

Aims: Neovascularisation in resolving venous thrombi is associated with a rise in the expression of vascular endothelial growth factor (VEGF). We aimed to determine whether increasing VEGF levels by adenovirus-mediated transfection of the VEGF gene enhances thrombus recanalisation and resolution.

Methods: Thrombus was formed in the inferior vena cava of rats. Adenovirus gene constructs encoding green fluorescent protein (ad.GFP) or VEGF (ad.VEGF) were injected into rat thrombus to confirm the location and the amount of VEGF expressed. Thrombi in 2 cohorts of rats, were injected either with ad.VEGF or empty virus (ad.0) [n=20/group].

After 7 days, thrombus size and recanalisation was measured by image analysis of sections. Circulating progenitor cells were enumerated using antibodies against CD45, Sca1 and VEGFR2.

Results: GFP expression was mainly detected in the vein wall at day 3 but by day 7 expression was also located in cells within the body of thrombus. VEGF protein expression peaked at 4 days $(376 \mathrm{pg} / \mathrm{mg})$. Treatment with ad.VEGF caused (i) $>50 \%$ reduction in thrombus size $(22.0 \pm 4.0 \mathrm{~mm} 2$ vs $47.7 \pm 5.1 \mathrm{~mm} 2, p=0.0005)$; (ii) $>3$-fold increase in recanalisation $(13.6 \pm 4.1 \%$ vs $3.9 \pm 0.69 \%, p=0.0003)$; increased thrombus macrophage content $(19.9 \%$ vs $12.8 \%, p=0.002)$ and (iv) almost doubled circulating progenitor cells $(75230 \pm 11696$ vs $34888 \pm$ $7235, p=0.014$ ) when compared with control.

Conclusion: The reduction in thrombus size and increase in recanalisation suggests that the ad.VEGF construct may be suitable as a novel treatment for DVT to promptly restore flow within a thrombosed vein and reduce post thrombotic complications.

0008

TOLL LIKE RECEPTOR 9 (TLR9) SIGNALING IN ENTEROCYTES ATTENUATES THE RESPONSIVENESS OF TOLL LIKE RECEPTOR 4 (TLR4) TO ENDOTOXIN IN THE PATHOGENESIS OF NECROTIZING ENTEROCOLITIS

S.C. Gribar, R.J. Anand, C.L. Leaphart, J.A. Cavallo, J.W. Kohler, T.D. Dubowski, J. Li, D.J. Hackam

University of Pittsburgh Medical Center, Pittsburgh, USA

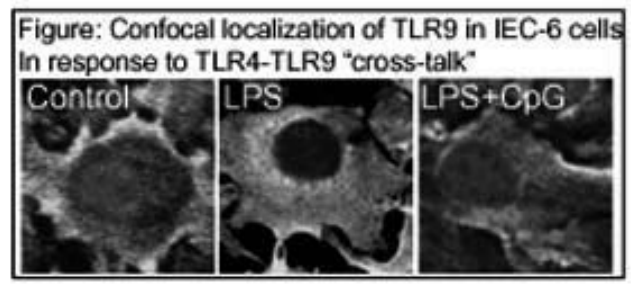

Introduction: Necrotizing enterocolitis (NEC) is characterized by inflammation of the intestinal epithelium, and is mediated in part through the activation of TLR4 on enterocytes by endotoxin leading to increased phosphorylation of p38 MAPKinase. Since enterocytes are exposed to endotoxin on a continuous basis, this raises the possibility that other toll like receptors could modulate the extent of intestinal inflammation. Previous authors have suggested that activation of toll like receptor 9 (TLR9) by bacterial DNA (CpG-DNA) may exert an anti-inflammatory effect on various organs, although a possible interaction between TLR4 and TLR9 remains largely unexplored. We now hypothesize that TLR9 activation could modulate TLR4-mediated signaling on the enterocyte in the pathogenesis of NEC, and sought to define the mechanisms involved. 
Methods: The expression of TLR4 and TLR9 on IEC-6 and HT-29 enterocytes was assessed by SDS-PAGE or quantitative real time PCR using specific primers. The localization of these toll receptors within enterocytes was determined by confocal immuno-fluorescent microscopy, while receptor activation was assessed as the expression of phosphop38. NEC was induced in newborn $\mathrm{C} 3 \mathrm{H} / \mathrm{HeOUJ}$ mice using the combination of hypoxia and enteric formula for four days, and intestinal mucosal scrapings were harvested for analysis.

Results: Enterocytes were found to express both TLR4 and TLR9 by SDS-PAGE and RT-PCR, which were expressed at the cell surface by confocal microscopy. Activation of enterocyte TLR4 by LPS ( 50 $\mu \mathrm{g} / \mathrm{ml}, 6 \mathrm{~h}$ ) led to an increase in pro-inflammatory signaling by IEC-6 enterocytes, as characterized by an increase in phospho-p38 expression. Treatment of IEC-6 cells with a combination of the TLR9 agonist CpG-DNA $(0.5 \mu \mathrm{M}, 6 \mathrm{~h})$ and the TLR4 agonist LPS led to an attenuation of LPS responsiveness, as characterized by a reduction in phospho-p38 expression. To understand the mechanism by which TLR9 activation could limit TLR4 responsiveness, treatment of IEC-6 cells with LPS caused the sequestration of TLR9 within the cytoplasm of IEC-6 cells. Treatment with CpG-DNA and LPS restored TLR9 expression on the cell surface, allowing for further receptor signaling (see Figure). Strikingly, newborn mice with experimental NEC demonstrated a significant increase in TLR4 and a corresponding decrease in TLR9 expression in the inflamed ileal mucosa, suggesting that "cross-talk" between these two receptors in vivo may contribute to the pathogenesis of NEC.

Conclusion: We conclude that TLR9 activation limits the degree of TLR4-induced signaling on the enterocyte, and that the sequestration of TLR9 by the TLR4 agonist LPS may in part mediate this effect. Further insights into the nature of the interaction between TLR4 and TLR9 on the enterocyte may provide novel therapeutic approaches for patients with NEC and other diseases of intestinal inflammation.

\section{O009}

FOLEY CATHETER BALLOON TAMPONADE FOR LIFE-THREATENING HEMORRHAGE IN PENETRATING NECK TRAUMA

C Troskie, P.H. Navsaria, M.Thomas, M. Edu S.Swart, A.J. Nicol Trauna Unit, Groote Schuur Hospital and Faculty of Health Sciences, University of Cape Town, South Africa

Background: Foley catheter(FC)balloon tamponade is a well-recognized technique employed to arrest hemorrhage from penetrating wounds. The aim of this study is to review our experience with this technique in penetrating neck wounds and to propose a management algorithm for patients with succesfull FC tamponade.

Methods: A retrospective chart review (July 2004-June 2005 inclusive)was performed of patients identified from a prospectively collected penetrating neck injury computer database in whom a FC balloon tampande was used.The units policy for penetrating neck injuries is one of selective non-operative management.All patients with successful FC tamponade underwent angiography.A venous injury was diagnosed if angiography was normal.Ancillary tests were performed as indicated. Removal of the FC was performed in the

operating theatre.

Results: During the study period, 220 patients with penetrating neck injuries were seen.FC balloon tamponade was used in 18 patients.It was successfull in 17 patients.Angiography was positive in 3 patients,all of whom underwent surgery. The FC was successfully removed in 13 patients at a mean of 72(range48-96) hours. One patient bled on removing the catheter, mandating emergency surgery was preformed.

Conclusion: Foley catheter balloon tamponade remains a useful adjunct in the management of selective patients with penetrating,bleeding neck wounds.

O011

THE EFFECTS OF ANTIBIOTIC PROPHLAXY AND MECHANICAL INTESTINAL CLEANSING ON PREVENTING BACTERIAL TRANSLOCATION DUE TO PRINGLE MANEUVER (EXPERIMENTAL STUDY)

M. Sahin, B. Erenoglu, Y. Tatkan, M. Baykan

Selcuk University Meram Medical Faculty, KONYA, Turkey

Aim: In this study we aimed to investigate the effects of mechanical intestinal cleansing plus antibiotic prophylaxy on preventing bacterial translocation during Pringle Maneuver in rabbits.
Material and methods: In this study 48 New Zelland male rabbits were used. The rabbits were fed by standart rabbit food and tap water under stable temperature of central heating in stainless steel cages. Rabbits were divided into four groups. Group I: control group, nothing was given, Group II: parenteral antibiotic group, $100 \mathrm{mg} / \mathrm{kg}$ Cefizoxim, Group III: intestinal cleansing group, with $180 \mathrm{cc}$ sodium phosphate and Group IV: intestinal cleansing and parenteral antibiotic group, the same procedur as group II and III. The rabbits were fasted on the nihgt prior to surgucal intervention day. A mixture of $100 \mathrm{mg} / \mathrm{kg}$ ketamine $\mathrm{HCL}$ and $10 \mathrm{mg} 2 \%$ xylazine $\mathrm{HCL}$ anesthesia was applied to rabbits. Laparatomy was done after shaving hair in the abdominal region and cleaning with polividon iodine. After laparatomy, $100 \mathrm{ml} / \mathrm{kg}$ 0.9\% NaCL solution was given via vena cava and then portal region was dissected and portal triad was turned by a clamp. In group I nothing was done, In other groups Pringle Maneuver was applied by a Satensky clamp for a 20-minute period. Blood samples from portal vein were taken before and after Pringle Maneuver for blood culture. After the maneuver, mesenteric lenf node samples were taken, and splenectomy was done for culture. The blood samples were inoculated into BacTecTM Blood culture tubes and incubated for 5 days. Lenf node and spleen samples were also inoculated into the same tubes as same manner. Bacteria types were identified in all positive culture samples.

Results: There was 34 positive results in Group I, 25 positive results in Group II, 17 positive results in Group III and 6 positive results in Group IV. All the treatment groups showed a meeningful differences from Control group. There was no meaningful differences between the groups II and III. Group IV has the superior results in all. E. Coli, E. Fecalis and Klebsiella were the most encountered microorganism consequtively. Conclusion: Antibiotic prophylaxy or mechanical intestinal cleansing can reduce bacterial translocation frequency but when these two modalities were used together the succes ratio was increasing.

0012

\section{TENSION-FREE REPAIR VERSUS BASSINI TECHNIQUE FOR} STRANGULATED INGUINAL HERNIA: A COMPARATIVE STUDY

M.A. Elsebae, M.S. Hedaya, H.E. Ezzat

Theodor Bilharz Medical Research Institute, Caïro, Egypt

By Evidence Based Medicine (EBM) principles, several meta-analyses concluded that use of mesh is superior to the non-mesh operations in inguinal hernia surgery. Wound infection is a potential complication of all hernia repairs and deep-seated infection involving an inserted mesh may result in chronic groin sepsis. In the event of incarcerated or strangulated hernias, however; placement of prosthetic material is presumed to increase that risk of infection. Aim of the study is to compare the outcome of tension-free mesh repair to Bassini technique used to treat strangulated inguinal hernia. In the period 2002-2006, 75 patients were submitted to emergency operation because of strangulated inguinal hernia. 33 patients underwent open tension-free anterior repair utilizing a monofilament polypropylene mesh (group A) according to Lichtenstein 'tension free' technique, whereas the remaining 42 patients underwent Bassini technique (group B). Selective policy was adopted in choosing our patients for mesh herniorrhaphy for strangulated inguinal hernia. It was applied only for those with a simple strangulated inguinal hernia. Assessment of the primary outcome included surgical complications and hospital stay and secondary outcome was the recurrence of hernia at an average one year. Postoperative complication rate did not differ significantly between the two groups $(5 / 33,15.1 \%$ vs. $5 / 42,11.9 \%, p=N S)$. Postoperative hospital stay was also significantly longer in group $B$ compared to group A $(10.3+/-3.4$ days vs. $4.5+/-2.1$ days, $p<0.01$ ). During the follow-up, (mean $12+/-4.2$ months), three patients had recurrence after Bassini operation (group B), but there was no recurrent hernia after mesh herniorrhaphy (group $A)(0 / 33=0 \%$ vs $3 / 42=7.14 \%, p<0.01$ ). It was concluded that the use of tension-free polypropylene mesh repair in emergency treatment of simple strangulated inguinal hernia is safe, simple, effective and without recurrence.

\section{O013}

\section{CHARACTERISTICS OF APPENDECTOMIES OPERATED}

\section{ON HOLIDAYS}

Y. Miura, K. Hirayama, Y. Nakajima, K. Saitoh, S. Yomoyuki, S.Tukamoto, Y. Enomoto

Hiraka General Hospital, Yokote Akita, Japan 

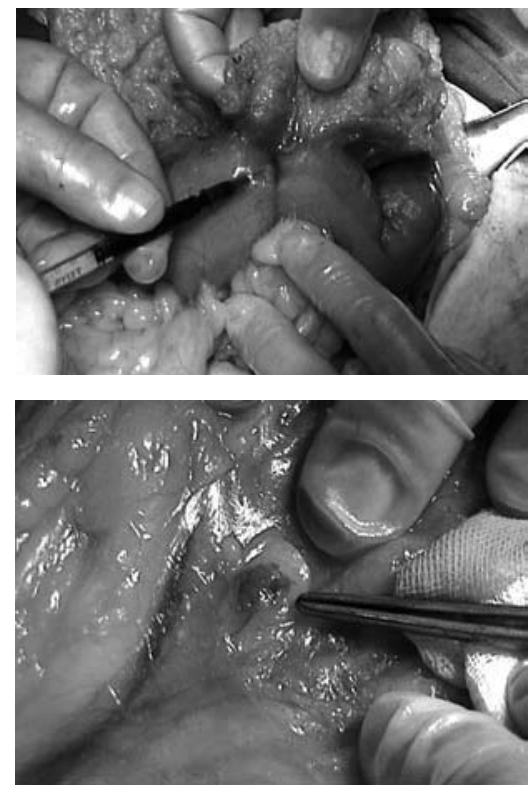

Introduction: Acute appendicitis is one of the common diseases for abdominal surgeons. Many appendectomies are performed in emergency situation. Some emergency appendectomies are performed even on holidays. Holiday operations may be less beneficial than usual weekday operations because of holiday situation in a hospital.

Methods: We investigated the characteristics of appendectomies operated on holidays in contrast to appendectomies operated on usual weekday. Check points are patient backgrounds, histological findings, surgical procedures, complications and postoperative hospital periods. Results: In our investigation, holyday means Saturday, Sunday and National Holiday etc. Weekday means Monday ' Friday when our hospital works as usual. We performed 144 appendectomies for acute appendicitis past 3 years (from Jan/2003 to Dec/2005). One patient who died in hospital suffered with DIC was excluded. Number of patients in Weekday Group and Holiday Group was 103 and 40. Mean age in each group was 46.3 and 37.4 years old $(p=0.0228, p<0.05)$, male patients were $67(65.0 \%)$ and $27(67.5 \%)(p=0.78, N S)$ and Body Mass Index (BMI) was 21.5 and $22.2(p=0.22$, NS). Pre-operative symptomatic period was 2.26 and 1.63 days $(p=0.0371, p<0.05)$ in Weekday and Holiday group. Gangrenous appendicitis was found in $42(40.8 \%)$ and $21(52.5 \%)(p=0.21, N S)$. Operation time in Weekday Group and Holiday Group was 58.8 and $54.4 \mathrm{~min}(\mathrm{p}=0.17, \mathrm{NS})$, bleeding volume was 37.8 and $30.7 \mathrm{ml}(p=0.26, \mathrm{NS})$, appendectomies which needed other organ resection were $9(8.74 \%)$ and $0(0 \%)(p=0.0168, p<0.05)$. The time when the operations were started was 15.5 and 12.4 o'clock on 24 hour basis $(p<0.001)$. SSIs were found in $13(12.6 \%)$ and $5(12.5 \%)$ in Weekday and Holiday group ( $p=0.94, N S)$, infections other than SSI were $3(2.91 \%)$ and $1(2.50 \%)(p=0.90, N S)$. All GI tract complications were found in $8(7.77 \%)$ and $1(2.50 \%)(p=0.21, N S)$ in Weekday and Holiday Group, respiratory complications were $2(1.94 \%)$ and $0(0.00 \%)$ $(p=0.32, N P)$. Postoperative hospital stay was 9.87 days and 7.58 days $(p=0.0233, p<0.05)$.

Conclusion: Appendectomies operated on holidays were ironically more beneficial than weekday appendectomies. We could start the operation earlier on holidays than on weekdays. Weekday appendicitis needed other organ resection. Holyday group showed shorter hospital stay. This benefit of holiday group can depend on the age and appendicitis characteristics. The age of holiday group was younger. Younger patients are likely to consult emergency hospital than older patients. Some patients in weekday group needed other organ resection. This can mean the delay of diagnosis and spread of infection especially in older patients. In these patients, appendicitis showed so-called tight inflammation stage which needed other organ resection. The priority is thought to be early diagnosis and early operation in the case of appendectomy especially in older patients. We are also investigating the efficacy of ultrasound examination and CT for the diagnosis of appendicitis. These examinations might to be useful for the early diagnosis of acute appendicitis. In our opinion, we should operate as soon as possible, if the patient has any suspicious sign of acute appendicitis.
0014

MANAGEMENT OF ACUTE PANCREATITIS : WHERE DO WE STAND?

R. Sridhar ${ }^{1}$, M. K. Jha², S.A. Debrah

1 Darlington Memorial Hospital, Darlington, United Kingdom

2 The James Cook University Hospital, Middlesbrough, United Kingdom

Introduction: Management of Acute Pancreatitis (AP) continues to remain a major problem despite set guidelines from The British Society of Gastroenterology1. Practices vary from hospital to hospital and so do the outcome. An audit of existing practice in our hospital was undertaken to determine if these were in accordance with published guidelines by The British Society of Gastroenterology (BSG).

Methods:146 patients (152 episodes) who were admitted an emergency basis with a diagnosis of AP2 (amylase 3 times the normal) between April 2003 and September 2004, included in the study. The case notes of all these patients were retrieved from the hospital database. A sub-group of patients fulfilling the following criteria were identified: if $f^{1 / 4}$ A hospital stay of more than 7 days

if $1 / 4$ A referral to a tertiary centre

if $1 / 4$ Death during the stay in the hospital.

54 patients were considered suitable for further analysis. MGS and CRP were used for severity assessment. The patients who had MGS of 3 or more were categorised as severe AP\&H\&3\&\&/H.

Results: A slight male preponderance $(52 \%)$ was noted among these 54 patients. Median age (60 years) and length of stay was 14 days. Only $11 \%$ of these patients had the required investigations for severity stratification within 24 hours, this improved to $40 \%$ at 48 hours. Ninety percent of the patients had their CRP measured in the first 48hrs, of which only $14(26 \%)$ showed a level of $150 \mathrm{mg} / \mathrm{dl}$ or more. Fourteen (26\%) patients underwent CT scan between days 3 and 10 of which only 8 met positive criteria on severity scale. Twelve patients (22\%) were classed as severe according to MGS however; only 5 of them needed HDU support. On the other hand a total of 12 out of 54 patients required HDU / ITU based on their clinical needs, of which only 7 had MGS of 3 or more. Two patients out of 12 (16.6\%) died after developing multiple organ failure.

Conclusion: This study demonstrates deficiencies in severity stratification and documentation. Recommendations were made and changes have been implemented to improve the shortcomings. Printed pathways detailing the parameters of MGS have been designed which are to be inserted in the patient's case notes immediately following the diagnosis. Emphasis should also lie on the clinical status of the patient regardless of any objective scoring system in determining the level of support required by the patients. It remains to be seen whether these new measures improve the compliance. We plan to re-audit this in the near future. References:

1. British Society of Gastroenterology Working Party. United Kingdom guidelines for the management of acute pancreatitis. Gut 1998; 42 (suppl 2); S1-S13. 2. A clinically based classification system for acute pancreatitis. Summary of the International Symposium on Acute Pancreatitis, Atlanta, Ga, September 11 through 13, 1992. Arch Surg. 1993 May;128(5):586-90. Bradley EL 3rd. 3. Blamsey SL, Imrie CW, et al. Prognostic factors in acute pancreatitis. Gut. 1984;25:1340-1346

\section{O015}

EFFORTS TO IMPROVE OUTCOME AFTER COMBINED CYTOREDUCTIVE SURGERY AND RADIOIMMUNOTHERAPY FOR EXPERIMENTAL PERITONEAL CARCINOMATOSIS: THE USE OF HYPERTHERMIA AND RECOMBINANT TISSUE PLASMINOGEN ACTIVATOR

F. Aarts, T. Hendriks, O.C. Boerman, R.P. Bleichrodt UMCN St. Radboud Nijmegen, Nijmegen, The Netherlands

Background and aim: Peritoneal carcinomatosis (PC) of colorectal origin is currently treated by cytoreductive surgery and, as an adjuvant, hyperthermic intraperitoneal chemotherapy, which results in a high morbidity and mortality. Previously we demonstrated that radioimmunotherapy (RIT) is a highly efficient adjuvant to cytoreductive surgery (CS) in a preclinical model of PC. Although RIT significantly increased survival, animals still developed intraperitoneal tumours in time. One reason could be that postoperative fibrin depositions hampered penetration of the radioactive antibody. Therefore, we have tried to improve the efficiency of RIT by combining it with fibrinolytic treatment. Since 
experimental evidence in other tumour models has demonstrated that hyperthermia can improve the efficiency of RIT, we have also tested such treatment in our model.

Materials and methods: PC was induced by intraperitoneal inoculation of CC531 colon carcinoma cells in Wag/Rij rats. In experiment 1 , animals were subjected to CS only, CS+whole body hyperthermia (WBH), $\mathrm{CS}+\mathrm{RIT}$ or $\mathrm{CS}+\mathrm{WBH}+\mathrm{RIT}$ (CS+HRIT). WBH was induced by housing the rats at $400 \mathrm{C}$ for 3 hours immediately after surgery and the administration of RIT. In experiment 2, rats were subjected to CS, CS followed by the intraperitoneal administration of recombinant tissue plasminogen activator (rtPA), at a dose of $1.25 \mathrm{mg}$ twice daily for 3 days, CS+RIT and CS followed by RIT and rtPA. RIT consisted of $74 \mathrm{MBq} 177 \mathrm{Lu}$ - labelled anti-CC531 antibody MG1. The primary endpoint was survival. In addition, the effect of hyperthermia on both the biodistribution of the radiolabeled antibody and the hypoxic fraction of intraperitoneal tumour nodules was determined.

Results: In general, all treatments were well tolerated, although two animals died directly following the hyperthermia treatment. In experiment 1 , the median survival of animals in the $\mathrm{CS}$ and $\mathrm{CS}+\mathrm{WBH}$ groups was 34 days and 37 days, respectively $(P=0.28)$. Median survival of the $C S+R I T$ or $\mathrm{CS}+\mathrm{HRIT}$ was 63 days and 86 days $((\mathrm{P}<0.0003$ and $\mathrm{P}<0.0006$ compared to CS), respectively. No difference was found between the two latter groups $(P=0.13)$. No differences were found in tumour uptake between animals treated with hyperthermia or controls. Moreover, no differences were found in tumour hypoxia after hyperthermia. In experiment 2, median survival after CS and CS+rtPA was 50 days and 42 days, respectively $(P=0.1)$. Median survival for $C S+R I T$ was 106 days and for CS+RIT+rtPA it was 103 days (both $\mathrm{P}<0.0001$ compared to the groups without RIT). No difference was found between CS+RIT and CS+RIT+rtPA $(P=0.83)$.

Conclusion: The application of whole body hyperthermia or rtPA in combination with adjuvant RIT after CS for the treatment of PC of colonic is feasible, but does not potentiate the efficacy of RIT.

O016

\section{LIPID-MEDIATED HUMAN IL-10 GENE TRANSFER TO THE AIRWAY OF RAT LUNG ALLOGRAFT FOR PREVENTION OF ALLOGRAFT} REJECTION

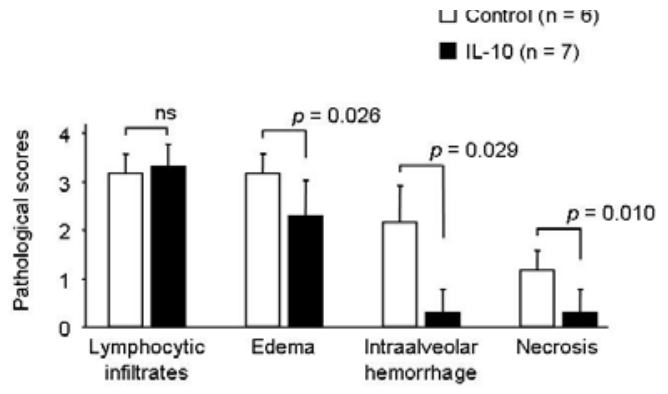

Picture 1: Pathological scores

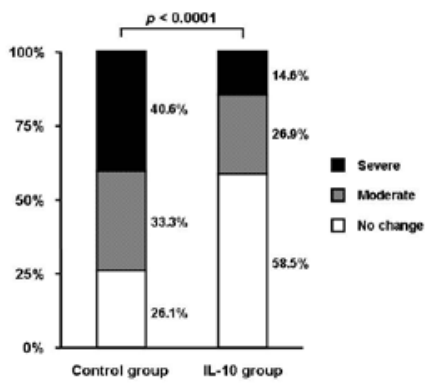

Picture 2: The intensity of bronchiolar epithelial loss

H. Oishi, Y. Okada, T. Kikuchi, T. Sado, Y. Hoshikawa, M. Noda, Y. Matsumura, T. Kondo

Institute Of Idac, Tohoku University, Sendai, Japan

Background: Although interleukin-10 (IL-10) gene transfer has been shown effective in modifying allograft rejection (AR) in some animal models of organ transplantation, the effect of transbronchial transfer of IL-10 gene on lung AR has not been defined. We examined the effect of ex vivo lipid-mediated transbronchial human IL-10 (hIL-10) gene transfer on acute $A R$ in a rat model of lung transplantation.

Materials and methods: Lewis and Brown Norway rats were used as recipients and donors, respectively. We used the pCMVbeta, a plasmid encoding $E$. coli beta-galactosidase, for control group $(n=6)$ and the pCMVhIL-10, a plasmid encoding hIL-10, for IL-10 group $(n=7)$. The harvested donor lungs were intra-bronchially instilled with lipid-pCMVbeta complex solution or lipid-pCMVhIL-10 complex solution, and then orthotopically transplanted into recipients. Six days after transplantation, allografts were removed and hIL-10 gene expression was examined by reverse transcriptase-polymerase chain reaction. The allografts were also pathologically examined and several pathological categories of inflammation (lymphocytic infiltrates, edema, hemorrhage, necrosis) associated with lung AR were scored on a scale of 0 - 4 . The intensity of epithelial injury of all bronchioles in the allografts was also examined and categorized into one of the followings; no change, 1- $50 \%$ of epithelial loss (moderate), 50 - 10\% of epithelial loss (severe).

Results: The hIL-10 mRNA expression was readily detected in all allografts of the IL-10 group and not in the control group. Although pathological scores for lymphocytic infiltrates were comparable between the both groups, scores for edema ( $2.3 \pm 0.8$ vs. $3.2 \pm 0.4)$, hemorrhage $(0.3 \pm 0.5$ vs. $2.2 \pm 0.8)$ and necrosis $(0.3 \pm 0.5$ vs. $1.2 \pm 0.4)$ were significantly reduced in the IL-10 group compared with the control (Figure $1, p<0.05$, respectively, with Mann-Whitney $U$ test). The intensity of bronchiolar epithelial loss was significantly decreased in the IL-10 group (no change: $58.5 \%$, moderate: $26.9 \%$, severe: $14.6 \%$ ) compared with the control group (no change: $26.1 \%$, moderate: $33.3 \%$, severe: $40.6 \%$ ) (Figure 2, p 0.0001 with chi-square test).

Conclusion: The present study showed that lipid-mediated transbronchial IL-10 gene transfer significantly improved the intensity of acute inflammation associated with lung $A R$ and also retained morphology of bronchiolar epithelial cells, which may lead to a novel therapeutic modality to treat bronchiolitis obliterans syndrome.

\section{7}

\section{IMPACT OF ACUTE REJECTION EPISODES ON CHRONIC ALLO- GRAFT NEPHROPATHY DEVELOPMENT}

L.M.F. Fabris ${ }^{1}$, A.F.B. Aiello², L.F. Furian"1, B.E. Ekser ${ }^{1}$, N.B. Baldan, M.V. Valente ${ }^{3}$, P.R. Rigotti ${ }^{1}$

1 Kidney And Pancreas Transplantation Unit, Padova, Italy

2 Department Of Oncology And Neuroscience, Chieti, Italy

3 Institute Of Pathological Anatomy, Padova, Italy

Background: Chronic Allograft Nephropathy (CAN) is the most frequent cause of late graft failure in kidney transplant recipients. The occurrence of acute rejections is a risk factor for development of CAN and the severity of histological lesions may play a role in CAN. Aim of this study was to evaluate clinical and histological parameters that may be relevant for the development of CAN in patients with biopsy proven episodes of acute rejection.

Design: From 1988 to 2000, 216 renal transplant recipients performed at our Center have been retrospectively evaluated (mean follow up: $203.5+/-42.5$ months). Immunosuppression was based on cyclosporine in all cases. Of these 216 patients 137 did not experience any episode of acute rejection (group 1) whereas 79 experienced one or more biopsy proven acute rejection episodes, classified for type and grade according to the Banff ' 97 classification (group 2). The two groups were compared in terms of renal function and the occurrence of CAN, evaluated according to the Banff ' 97 classification. Clinical and histological parameters that could significantly correlate with CAN were then assessed within the group 2 patients.

Results: Mean serum creatinine in group 1 at 6 months, 2 and 5 years after transplantation was lower than in group $2(p<0.001)$. In group 2 the number of patients that developed CAN $(27 / 79=34.2 \%)$ was higher than in group $1(16 / 137=11.7 \%)(p<0.0001)$ and graft loss due to CAN was also higher $(9 / 137=6,6 \%$ in group 1 vs $13 / 79=16.5 \%$ in group $2, p<0.02$ ), while no significant difference was observed in graft loss due to death among the two groups $(24 / 137=17 \%$ in group 1 vs $9 / 79=11.4 \%$ in group 2 ). Interestingly, CAN occurred later in group 1 (168.6 months, 95\%C.I. 156.6-180.4) than in group 2 (116.7 months, $95 \%$ C.I. 101.6-131.9) $(p<0.0002)$. In group 2 , considering the histologically more severe acute rejection episode per patient, 18 patients had a borderline rejection (1-4 mononuclear cells/tubular cross section), 30 had type IA (5-10 mononuclear cells/tubular cross section), and 29 had type IB rejections (> 10 mononuclear cells/tubular cross section). Only 2 cases showed a grade II rejection and were not considered. The histopathological degree of rejection correlated with development of CAN ( $p<0.02)$. Interestingly, it did not correlate with the mean \% increase of serum creatinine at the time of the biopsy. 
Among patients of group 2, HLA A, B, and DR matching was not significantly different. CAN was significantly associated with a late timing of rejection $(p<0.01)$, while it was not associated with age, male/female ratio, living/cadaveric donor ratio, cold ischemia time, occurrence of post-transplant tubular necrosis and mean \% increase of serum creatinine at the time of the biopsy.

Conclusion: CAN develops earlier in patients experiencing acute rejection episodes, moreover, the severity of the acute rejection episodes correlates with CAN development.

\section{O018}

\section{LIVER INTEGRITY AFTER WARM ISCHEMIA IN SITU AND BRIEF PRESERVATION EX VIVO: THE VALUE OF AEROBIC POST- CONDITIONING}

S. Manekeller, V. Dobberahn, A. Hirner, T. Minor

UKB, Division of Surgical Research, Bonn, Germany

Background: In face of the increasing shortage of donor organs for clinical transplantation, the donor acceptance criteria for liver retrieval have been expanded. Non heart beating donors (NHBD) may represent an alternative supply of organs and many centres have started programs for the procurement of livers from NHBD's. However, due to the immanent ischemic challenge upon organ harvest after cardiac standstil of the donor, these grafts are thought to be particularly endangered by a reduced tolerance to ulterior preservation.

We evaluated the respective effects of warm ischemic injury in nonheart-beating donor (NHBD) grafts and/or cold ischemia time on liver viability. Eventually, the restorative potential of oxygenated hypothermic perfusion after cold storage should be investigated.

Methods: Livers were retrieved from male Wistar-rats and preserved with HTK-solution for $6 \mathrm{~h}$ or $18 \mathrm{~h}$ by cold storage (CS). Organ retrieval took place either from heart beating donors (HB) or 30 min after cardiac arrest from non heart beating donors (NHBD). In one group of NHBDlivers the attempt was made to enhance tissue viability by restoration of energetic homeostasis through oxygenated machine perfusion with oxygenated HTK in a recirculating system. This period of 'postconditioning' was limited to $1 \mathrm{~h}$ and was added to the total preservation time.

Viability of all livers was evaluated thereafter upon reperfusion in vitro according to previously validated techniques in a recirculating system for $120 \mathrm{~min}$ at $37^{\circ} \mathrm{C}$ with oxygenated $(95 \% \mathrm{O} 2-5 \% \mathrm{CO} 2$; pO2> 500 $\mathrm{mmHg}$ ) Krebs-Henseleit buffer at a constant flow of $3 \mathrm{ml} / \mathrm{g} \times \mathrm{min}$. Bovine serum albumin was added to the perfusate at a concentration of $3 \mathrm{mg} \%$. Results: Compared to $6 \mathrm{~h}$ CS of HB-livers, enzyme leakage (GPT, LDH) and functional recovery (oxygen consumption, ammonia clearance, bile production) upon warm reperfusion were slightly disturbed after $18 \mathrm{~h}$ of HB-livers. but massively deteriorated after 18h CS in NHBD's $(p<0.05$ or more). By contrast, 6 h CS of NHBD resulted in an only limited impairment of all parameters which was found quite similar to the results in ctrl. after 18h CS. Induction of cellular apoptosis (cleavage of Caspase 3 and PARP) was found equally influenced by preceding warm ischemia (NHBD) or extended times of CS, but significantly triggered only by the combination of both events. After $6 \mathrm{~h}$ of CS, 1 hour of oxygenated hypothermic machine perfusion ('post-conditioning) was able to bring the performance of NHBD-liver into line with the controls.

Conclusion: It is concluded that a limited time of warm ischemia in the donor only multiplied graft injury after long term CS, but does not need to preclude acceptable results if reperfusion is initiated after short periods of CS. Additional postconditioning by oxygenated perfusion in the cold prior to transplantation may represent an additional tool to improve tissue integrity in those marginal grafts. Moreover, conditioning by oxygenated machine perfusion prior to implantation may also help to judge liver viability.

\section{O019}

\section{IMPACT OF STEROIDS ON HEPATITIS C VIRUS REPLICATION IN VITRO}

L.J.W. van der Laan, S.D. Henry, J. Van Dijck, H.J. Metselaar, H.W. Tilanus Erasmus Medical Center Rotterdam, Rotterdam, The Netherlands

Background: Chronic hepatitis $\mathrm{C}$ virus (HCV) infection is the leading indication for liver transplantation (Tx) worldwide. The success of $\mathrm{Tx}$ is often compromised by a rapid re-infection of the graft due to persistent virus. Many factors have been implicated in the increased severity of recurrence, including immunosuppressant regimes. Steroids are universally administered during liver Tx and are often used as low dose maintenance immunosuppression. Clinical evidence suggests that steroids boluses used to treat acute rejection are associated with an in increase in HCV viral load and the severity of recurrence. The aim of this study was to determine the direct effect of steroids on the replication of $\mathrm{HCV}$, in vitro, to better understand their effects on $\mathrm{HCV}$ recurrence after liver $\mathrm{Tx}$.

Methods: The effect of the steroids Dexamethasone (Dex) and Prednisolone (Pred) were tested in vitro using an HCV-replicon model. $\mathrm{HCV}$ replication was assessed based on luciferase reporter expression (luminescence) and HCV RNA (RT-PCR). As controls, cell proliferation, cell death and total protein content were determined.

Results: In short-term (18hr) experiments, at clinically relevant concentrations (1-10 nM), both Dex and Pred appeared to slightly increase $\mathrm{HCV}$ replication at $5 \mathrm{nM}$ concentration. Steroids also increased the total protein content of replicon cells. After normalizing the luciferase expression for total protein, Dex and Pred actually seem to slight reduce $\mathrm{HCV}$ replication. This minor reduction of HCV replication was confirmed by RT-PCR showing over $20 \%$ lower HCV RNA levels due to steroids. Neither steroid had an affect on the viability of replicon cells within short term cultures, though both Dex and Pred significantly reduced cell proliferation at the highest concentration tested.

Conclusion: Despite clinical evidence that the use of steroids aggravates recurrence of $\mathrm{HCV}$, our in vitro experiments show that steroids do not specifically enhance HCV viral replication. These findings suggest that the accelerated HCV recurrence after liver Tx is more likely due to steroid mediated effects on the anti-viral immune response.

\section{O020}

\section{INTRAVENOUS IMMUNOGLOBULINS REDUCE ALLOGENEIC} T-CELL ACTIVATION AFTER LIVER TRANSPLANTATION BY MODULATING THE INTERACTION BETWEEN DENDRITIC CELLS

\section{AND NK-CELLS}

T. Tha-In, H.J. Metselaar, J. Kwekkeboom, Z.M.A. Groothuismink, E.J. Kuipers, R.A. De Man, G. Kazemier, H.W. Tilanus

Erasmus Medical Center, Rotterdam, Rotterdam, The Netherlands

We have shown that intravenous immunoglobulins (IVIg) reduce the incidence of acute rejection after liver transplantation from $31 \%$ to $13 \%$ and suppress the allogeneic T-cell priming by dendritic cells (DC). Here, we investigated the mechanism by which IVIg prevent immune activation after liver transplantation.

Human DC, NK-cells and T-cells were isolated from blood of healthy individuals. DC were stimulated with TNF $\alpha /$ IL $1 \beta$ in absence or presence of IVIg. Thereafter, IVIg were removed and allogeneic NK-cells were added. NK-cell phenotype and apoptosis of DC were determined by flowcytometry. T-cell priming capacity of DC was assessed by culturing $\mathrm{DC}$ with allogeneic T- and/or NK-cells using $3 \mathrm{H}$-thymidine incorporation and CFSE-dilution techniques. In vivo changes in leukocyte population were monitored in patients after IVIg-treatment.

DC maturated in presence of IVIg (IVIg-DC) activated allogeneic NKcells and increased their interferon $y$ production compared to CTRLDC. Subsequently, the activated NK-cells induced apoptosis of IVIg-DC, as shown by increased Caspase-3 expression and increased 7-AAD staining (IVlg: $33 \pm 9 \%$ positive DC, CTRL: $17 \pm 8 \%, p<0.01$ ). When cultured with allogeneic T- and NK-cells, IVIg-DC were impaired in their allogeneic T-cell priming capacity by $81 \% \pm 15(p<0.05)$ compared to CTRL-DC. This is due to NK-cell mediated Antibody Dependent Cytotoxic Killing (ADCC) of IVIg-DC, which can be abrogated by blockade of Fc y RIII on NK-cells. By using monoclonal human IgG as a control, we identified that $A D C C$ is caused by dimers in IVIg preparations. Furthermore, IVlg-DC promoted expansion of CD56brightCD16-CCR7+ lymph node type NK-cells. In patients, NK cell numbers dropped at day 1 and increased at day 7 after IVIg bolus had been given.

IVIg reduce the incidence of acute rejection after liver transplantation by promoting NK-cell mediated ADCC of DC, which subsequently reduces the allogeneic T-cell priming. By modulating the early control switch of allogeneic T-cell priming, IVIg can prevent T-cell activation, and may therefore be a promising candidate for future non toxic immunosuppressive regimen after liver transplantation.

\section{O021}

META-ANALYSIS AND SYSTEMATIC REVIEW OF THE RELATIONSHIP BETWEEN VOLUME AND OUTCOME IN ABDOMINAL AORTIC ANEURYSM SURGERY 
P.J.E. Holt ${ }^{1}$, J.D. Poloniecki², I.M. Loftus ${ }^{2}$, M.M. Thompson ${ }^{2}$ St George's Vascular Institute, London, United Kingdom

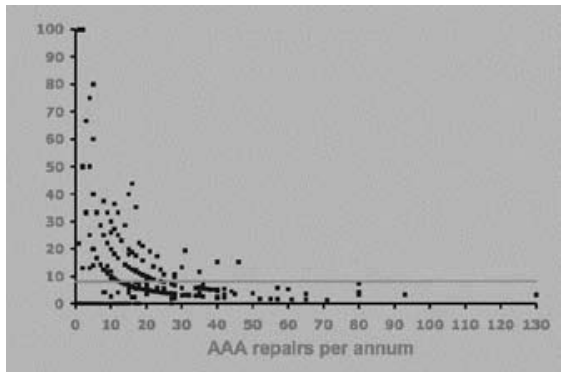

Picture 1: Mortality rate vs volume elective $A A A$ repairs

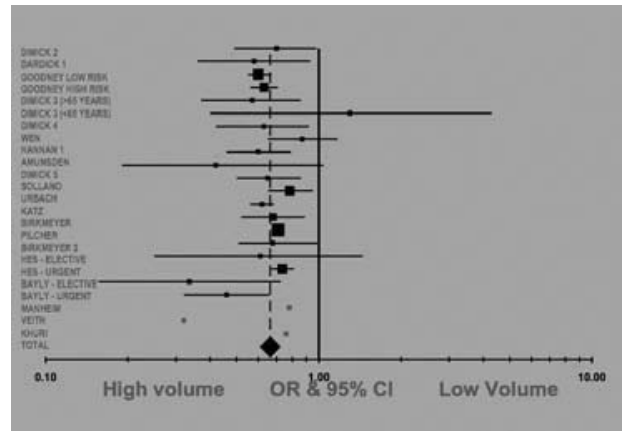

Picture 2: Forest Plot

Objectives: This study investigated the volume-outcome relationship for abdominal aortic aneurysm surgery and quantified critical volume thresholds.

Methods: PubMed, EMBASE and the Cochrane library were searched for articles on the volume-outcome relationship in elective and ruptured AAA surgery. UK data were included by analysis of the Hospital Episode Statistics (HES). Elective and ruptured AAA repairs were considered separately. The review conformed to the QUOROM statement. The data were meta-analysed and the odds ratios and 95\% confidence intervals for mortality at higher- and lower-volume hospitals were compared. Volume thresholds were identified from each paper.

Results: Analysis was of 421299 elective AAA and 45796 ruptured AAA. Significant relationships between mortality and annual volume were seen for both groups. Overall, the weighted odds ratio was 0.66 (0.65-0.67) for elective AAA repair at a threshold of 43 AAA per annum and 0.78 (0.73-0.82) for ruptured AAA repair at a threshold of 15 AAA per annum, both in favour of high volume units.

Conclusion: Significantly lower mortality with higher annual volumes of AAA surgery was demonstrated for both elective and ruptured AAA repair. These results suggested that AAA surgery should be performed only at higher volume centres.

\section{O022}

AN APPRAISAL OF ICODEXTRINA SOLUTION IN THE PREVENTION AND DEVELOPMENT OF INTRA-ABDOMINAL ADHESIONS. AN EXPERIMENTAL STUDY

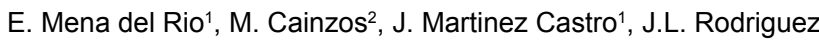
Couso $^{1}$, A. Laya Barca ${ }^{1}$, M. Amor Berdullas ${ }^{1}$

1 C. Hospitalario Universitario Santiago, Santiago de Compostella, Spain

Background: The development of intra-abdominal adhesions accompanied by both an increased morbility and even mortality, if a reintervention is necessary, is one of the most recurrent and important complications in abdominal surgery. Due to their capacity to completely cover the peritoneum surface at both the visceral and the parietal levels, interest has recently focused on the value of the intra-abdominal administration of anti-adhesion fluids on completion of a surgical intervention.

Aim: The aim of this study was to assess the efficacy of icodextrina solution in the reduction of the formation of intra-abdominal adhesions during a thirty day period subsequent to doing a cholecystectomy using a laparotomy in pigs.

Materials and methods: 28 pigs with a weight of between $23 \mathrm{~kg}$ and 27 $\mathrm{kg}$ were used. The animals were divided into two groups.

Group I. (Control group of 10 animals.) Under general anaesthesia and skin disinfected with iodine povidone, a laparotomy of $10-12 \mathrm{~cm}$ in length was performed on the right subcostal. A cholecystectomy using the standard technique was carried out through it. Next, the abdominal wall was closed at two levels with Vycril 0 while skin closure was made with staples. Group II. (Study group of 18 animals.) The same technical procedure as in group I was followed. On termination of the exeris of the gallbladder, $500 \mathrm{cc}$ of icodextrian solution at $4 \%$ (Adept $\mathrm{O}$ ) at $37^{\circ} \mathrm{C}$ was introduced into the abdominal cavity. So that an analysis of the development of intra-abdominal adhesions could be made, a medium laparotomy under general anaesthesia was performed after 30 days. The number of adhesions, their extension, consistency and vascularization were taken into account. Samples of the adhesions were taken for a histological study. The animals were sacrificed by the anaesthetist. The statistical analysis was made by using the $\mathrm{C} 2$ test $(p \leq 0.05)$.

Results: Group I. $100 \%$ of the ten animals studied developed intra-abdominal adhesions, which were multiple in all cases.

Group II. 17 (94.4\%) of the 18 animals studied developed post-operative intra-abdominal adhesions ( $p=N S$ ), which were multiple in 14 pigs $(82.4 \%)$. Most of them were located either between the liver and the parietal peritoneum, between the omentum and the gallbladder bed, or between the omentum and the abdominal wall. Additionally, adhesions were also found between the omentum or the small intestine and the metallic clips placed in the cystic duct, as well as between the spleen and the abdominal wall.

Conclusion: This experimental study has demonstrated the ineffectiveness of icodextrina solution at $4 \%$ in the prevention of the development of intra-abdominal adhesions after performing a cholecystectomy in pigs.

\section{O023}

\section{GENETIC BACKGROUND PROVIDING FOR PTC SUSCEPTIBILITY IN BENIGN MULTINODULAR THYROID DISEASE}

F.W. Weber ${ }^{1}$, M. Aldred, C.E. Broelsch 1 , A. Frilling ${ }^{1}$, C. Eng ${ }^{3}$

1 University of Essen, Essen, Germany,

3 Cleveland Clinic, Cleveland, USA

Background: The prevalence of incidental papillary thyroid microcarcinoma (mPTC) in patients treated for multinodular goitre is reported between 5 to $10 \%$. In a setting of multinodular disease (with or without a dominant nodule), the potentially malignant lesion can easily be missed during pre-operative fine needle aspiration biopsy. With this study, we addressed the question if multinodular goitre harboring an unsuspected PTC contains a background genetic signature that is associated with cancer development and might therefore indicate the presence of the malignant lesion even if the cancer nodule is not directly analyzed. The identification of such a 'susceptibility profile' could substantially influence and alter management of patients with this disease

Methods: 23 hyperplastic thyroid nodules from 23 patients that underwent surgery for multinodular disease have been analyzed. Of these, 12 patients were diagnosed with an incidental PTC on final histology. We utilized the Affymetrix HG-U133A GeneChip for global gene expression analysis. Data mining was done with established statistical methods. Results: Based on a 233 gene signature over $80 \%$ of all samples could be correctly identified. Notably, the most over-expressed gene in the MG+PTC group (3.6 fold, $p<0.0001$ ) was CDC42. It is known that JNK activation by RET is mediated by small, GTPases, in particular by CDC42. Interestingly, the other gene over expressed in hyperplastic nodules of patients diagnosed with an unsuspected PTC was TIMP3 (2.53fold) the tissue inhibitor of metalloproteinase-3, which is known to be negatively correlated with advanced disease.

Conclusion: Thus, we have shown that there does exist a genetic profile associated with a subset of benign multinodular disease harboring occult PTC. Similar to the different genomic alterations contributing to PTC genesis, it appears, that this genetic background is heterogeneous. However, based on our whole genome approach we provide for the first time evidence that the prediction of unsuspected PTC might indeed be possible for at least some patients. The complex genetic observations will be discussed. 
O024

DEREGULATION OF HUMAN MICRORNAS IN DIFFERENTIATED THYROID CANCER AND BENIGN THYROID NEOPLASIA

F.W. Weber ${ }^{1}$, A. Frilling ${ }^{1}$, C.E. Broelsch ${ }^{1}$, C. Eng ${ }^{2}$

1 University of Essen, Essen, Germany

2 Cleveland Clinic, Cleveland, USA

Background: The pathogenesis of thyroid carcinoma and their molecular relationship to each other still remains unclear. Micro RNA's are a novel class of non-coding RNA's that can orchestrate the regulation of hundreds of target genes. The aim of this study was to provide a comprehensive overview of the miRNA expression in thyroid neoplasia. We compared the expression of miRNAs in follicular thyroid carcinoma, adenoma, papillary thyroid carcinoma and normal thyroid tissue in order to elucidate their role in thyroid carcinogenesis.

Methods: 12 FTC, 8 FA and 3 normal thyroid tissue has been used for a micro-RNA Chip analysis (235 miRNAs). These results were then compared to the published expression profiles of 15 PTC and 15 normal thyroid tissue analyzed on the same platform. The raw data from the chips were analyzed using established methods.

Results: When compared to normal thyroid tissue, 49,33 and 21 microRNAs were significantly deregulated in FTC, FA and PTC (fold change $>2, p<0.001)$. The most significantly deregulated miRNAs in FTC (miR-126, miR26a, miR-100) were similarly deregulated in FA. In fact, only 4 miRNAs (miR-197, miR-346, miR-328, miR-192) were significantly differentially regulated between FTC and FA. MiR-126 over expression is specific for follicular neoplasia and not observed in PTC. Of the 5 most significantly over expressed miRNAs in PTC (miR-221, miR-222, miR-146, miR-181a, miR-21) $(p<0.0001)$ miR-221 and miR222 showed also an over expression in FTC and FA. Only miR-146 over expression was uniquely observed in PTC.

Conclusion: With this study, we gained 2 important insights. First, we showed that deregulation of miRNAs already occurs similarly in benign and malignant follicular neoplasia, indicate that both neoplasias originate from the same background. Second, some of the most significantly deregulated miRNAs in PTC are also over-expressed in follicular neoplasia. Together, our data might indicate that many miRNAs might provide for a proliferative phenotype but only a limited set does indeed facilitates the malignant genotype contributing to differentiated thyroid carcinoma.

\section{$\mathrm{O} 025$}

REFLECTANCE SPECTROMETRY AS INTRAOPERATIVE ASSESSMENT OF PERFUSION IN RECTAL ANASTOMOSIS A FEASIBILITY STUDY

A. Karliczek ${ }^{1}$, D.A. Benaron², P.C. Baas ${ }^{1}$, A. van der Stoel ${ }^{1}$, T. Wiggers ${ }^{3}$, G.M. van Dam ${ }^{3}$

1 Martini Hospital Groningen, Groningen, The Netherlands

2 Spectros Coorporation, Portola Valley, USA

3 University Medical Center Groningen, Groningen, The Netherlands

Evaluation of microperfusion in colorectal anastomoses remains a challenging problem. Intraoperative assessment of anastomotic ischemia might be an important factor in the prediction, and therefore prevention, of anastomotic dehiscence. In this study we evaluated the feasibility of tissue oximetry using shallow-penetrating visible light (visible light spectroscopy[VLS]) to measure microvascular haemoglobin oxygen saturation (Sto2) in small, thin tissue volumes in rectal anastomoses in a standardized measurement protocol.

Materials and methods: VLS was evaluated in 11 rectal anastomotic procedures, all within $20 \mathrm{~cm}$ of the anal verge by using T-Stat ${ }^{\circledR}$ Ischemia Detection system (T-Stat 303) using a catheter probe. Feasibility was defined as number of planned measurements performed, stability of the assessments (expressed as descriptive statistics) and adverse events caused by the VLS-system or the protocol.

Results: Of 220 planned recordings, 125 (57\%) were carried out. Mucosal recordings showed a high standard deviation $(20,4-26,6)$, whilst serosal recordings showed less variation (SD 4,2-13,7). After ligation of mesenteric arteries, a decrease in saturation $(9 \%)$ of the rectal stump serosa was observed.

Conclusion: Based on the results of this preliminary study, VLS is an easy to perform and fast technique for intraoperative real-time assessment of microperfusion of the serosal surface in colorectal anastomosis. Additional multicenter studies evaluating VLS as a predictor of anastomotic leakage are currently being carried out.
O026

A STUDY ON THE SURVIVAL OF AN EXPERIMENTAL MODEL WITH INTRAPERITONEAL SEPSIS UNDERGOING LAPAROSCOPIC VS OPEN LAPAROTOMY

F. Karantonis ${ }^{2}$, D. Mantas ${ }^{1}$, N. Nikiteas ${ }^{1}$, E. Giamarellos ${ }^{3}$, D. Perrea ${ }^{4}$

1 Faculty of Medicine, Athens University, Athens, Greece

2 Laiko General Hospital, Athens, Greece

3 Attiko University Hospital, Athens, Greece

4 Experimental Labotatory, Medical Univers, Athens, Greece

Introduction: Laparoscopy, in contrast to laparotomy has proven to be the golden standard in various procedures, as it is a less stressful event in all means for the patient. A point of discussion though would be the comparison of the efficacy of these techniques in the treatment of intraabdominal sepsis.

Aim: The purpose of this study is to correlate the effect in the survival either of an open laparotomy or a laparoscopy procedure in an intraperitoneal sepsis environment.

Material: 29 male Wistar rats were separated and randomized in three groups. Anesthesia was achieved by using ether, ketamine and xylazine. Intraabdominal contamination was induced with $1 \mathrm{ml}^{*} 108 \mathrm{CFU}$ Pseudomonas aeruginosa. Group A $(n=9)$ was the control group in which we injected Pseudemonas aeruginosa intraperitoneal. In Group $B(n=10)$ we injected the same bacterial factor and after six hours we developed pneumoperitoneum. We used 3 catheters of $18 \mathrm{G}$ with one being connected to a $\mathrm{CO} 2$ insufflator maintaining a $0,21 / \mathrm{min}$ flow of $\mathrm{CO} 2$ and an intraperitoneal pressure of $5 \mathrm{mmHg}$. In Group C $(n=10)$ after the injection of the bacterial factor and the six hours interval we performed a $3 \mathrm{~cm}$ open laparotomy incision. Both interventions lasted for $30 \mathrm{~min}$. Vital signs, temperature and weight readings were taken at $0,6,7,8$, 10,12 hs and at 4 hour intervals after the 12th hour. Heart rate, respiratory rate and oxygen saturation readings were performed at $(0,6,8 \mathrm{hs})$. Every animal that died during the course of the experiment was subjected to autopsy, dissection and tissue harvesting. We harvested liver, spleen and lung tissue and conducted qualitative and quantitative bacterial cultures. Animals that survived were monitored for 144 hours and were subjected to euthanasia and tissue harvesting in order to perform bacterial cultures. Bacterial load, temperature, weight readings and hours of survival were subjected to statistical analysis using the SPSS package. The survival analysis was performed using the Kaplan-Meier formula.

Results: The statistical analysis of bacterial load levels between organ fragments did not reveal any statistical significant difference. The mean survival between the three groups was $9.44 \pm 0.45,51.1 \pm 19.28$ and $64.12 \pm 20.63$ hours respectively. There wasn't any statistical significant difference in the survival between the three groups except between Group $A$ and $C(p<0.0027)$. As concerning the other factors there was statistical significant difference in respiratory rate between Group $A$ and $B$ as well as between Group $A$ and $C$ and Group $A$ and $B$ concerning the oxygen saturation at 6 and 8 hours $(p=0.0080$ and $p=0.0020)$ respectively. Conclusion: According to this experimental model there is no statistical significant difference comparing laparoscopy vs open laparotomy concerning survival and visceral bacterial load. In addition, the $\mathrm{CO} 2$ application has no impact in the bacterial translocation. However the control animals would benefit if subjected to laparotomy in contrast to $\mathrm{CO} 2$ pneumoperitoneum.

\section{O028}

AUTOLOGOUS PLATELET GROWTH FACTOR GEL FACILITATES BONE SUBSTITUTE AND AUTOGENOUS BONE GROWTH IN A GOAT MODEL

P.A.M. Everts ${ }^{1}$, D. Delawi ${ }^{2}$, Chr. Brown Mahoney ${ }^{3}$, A. Van Erp ${ }^{1}$, E. Overdevest ${ }^{1}$, A .van Zundert ${ }^{1}$, J. Knape ${ }^{2}$, W. Dhert ${ }^{2}$

1 Catharina Hospital, Eindhoven, The Netherlands

2 University Medical Center, Utrecht, The Netherlands

3 Hamline University, St. Paul, USA

Introduction: Bone grafts are widely used in orthopedic surgery, maxillofacial surgery, and neurosurgery for the management of fracture nonunions, spinal fusion, and reconstructive surgery. Bone graft substitutes are considered to provide a functional substitute for autograft bone. Growth factors play an important role in this process by providing signaling for osteoinduction, through an osteogenic cell response to these signals. Platelets, essential in soft tissue healing and bone regeneration, 
are present in the blood clot that is formed at tissue injury sites of coagulation resulting in platelet degranulation. Autologous engineered platelet leukocyte gel (PLG) is composed of a high concentration of platelets, leucocytes, and thrombin and is a source of growth factors, which are normally, in-vivo, also present at tissue wound sites be applied locally to autograft bone or bone substitutes, to enhance bone regeneration due to the presence of a high concentration of platelet growth factors. PLG is prepared with so called table-top point-of care centrifuges.

Methods: Two three-compartment cages containing either autogenous bone or bi-calcium phosphate (BCP) or trabecular metal (TM), were implanted onto a goat spinal transverse processes $(n=10)$. One cage was immersed with PLG, the untreated cage served as a control. Histology and ffluorescent microscopy three fluorochrome labels were performed. Bone growth in these 3 compartments from PLG treated cages and controls, were studied with a histomorphometric analysis on sample sections. Furthermore, trans forming growth factor beta (TGF- ${ }^{2}$ ) analysis was performed.

Results: PLG treated cages had a significantly higher TGF_² concentration when compared to the circulating blood values. Compared to the control samples, bone growth in the PLG treated autogenous bone and BCP samples was significantly stronger. Relatively little bone growth in the TM samples and no differences in the PLG treated or untreated TM scaffolds were observed.

Conclusions and clinical relevance: This study demonstrates that autologous prepared platelet-leukocyte gel contains a high concentration TGF-2 ${ }^{2}$, and its application to autologous bone and BCP produced a quantifiable enhanced bone growth in comparison with non-treated grafts. The augmentation of bone defects by exogenous topical application of autologous derived platelet growth factors is a challenge. The results obtained from this goat model suggest a potential application for the addition of autologous prepared PLG in surgery for patients using autogenous bone graft or calcium phosphate scaffolds.

\section{O029}

\section{THE STARCH IN UNIVERSITY OF WISCONSIN PRESERVATION SOLUTION ENHANCES GENE THERAPY VECTOR DELIVERY}

L.J.W. van der Laan', S.D. Henry², H.J. Metselaar², P.G. van der Wegen ${ }^{2}$, B.J. Scholte ${ }^{2}$, H.W. Tilanus ${ }^{2}$

1 Erasmus Medical Center Rotterdam, Rotterdam, The Netherlands

Background: Isolated perfusion of the liver, like in the setting of transplantation, represents a unique opportunity for safe and effective targeting of gene therapies to improve transplant outcome, including the prevention of HCV recurrence. In the current study we examined different solutions used for graft preservation and determined their usefulness for lentiviral-based gene therapy delivery under hypo- and normo-thermic conditions.

Methods: Huh-7 hepatoma cells were suffused in preservation solutions at $4^{\circ} \mathrm{C}$ or $37^{\circ} \mathrm{C}$ containing GFP lentivectors at a multiplicity of infection of 0.5 . Universities of Wisconsin (UW), Histadine Tryptophan Ketoglutarate (HTK), EloHaes (EH), Na-PEG UW (IGL-1), or DMEM culture medium were tested. Transduction efficiency was determined by flowcytometry. Results: GFP positive cells could be observed with vector exposure times as short as 10 minutes in both normothermic and hypothermic conditions, with highest transduction achieved with UW and EH. After 2 hour incubation, transductions increased across all solutions in hy-

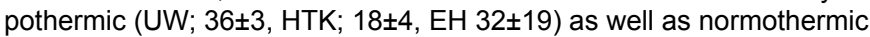
conditions (UW; 62 $\pm 6, \mathrm{HTK} ; 21 \pm 7, \mathrm{EH} 48 \pm 8$ ). To determine why the best transductions were achieved in UW, different components of UW were supplemented to HTK. Neither adenosine nor glutathione provided any increase to HTK's transduction potential, while the inclusion of hydroxyethyl starch (HES) significantly improved the percentage of GFP positive cells (UW; 46 \pm 4 , HTK; $20 \pm 4 ; 4 \%$ HES; $32 \pm 4,6 \%$ HES $41 \pm 5$ ). To rule out size exclusion (limited free liquid) as a possible mechanism of enhancement, the size exclusion agent polyethylene glycol (PEG) was tested via IGL-1 (PEG-UW) solution. The IGL-1 solution was slightly better than HTK with $27 \pm 3 \%$ transduction, though was not a significant increase. Conclusion: This study demonstrates superior vector delivery to hepatocytes using UW solution under conditions of hypothermic graft preservation. Starch based solutions, UW and EH, provided superior transductions over non-starch solutions (HTK and IGL-1), with as little as 10 minutes vector exposure. This demonstrates the feasibility of lentiviral gene delivery during the cold storage of liver grafts.
O030

IMPROVED AND ACCELERATED REGENERATION OF TRAUMATZED SKELETAL MUSCLE TISSUE BY ERYTHROPOIETIN

R. Rotter ${ }^{1}$, M. Menshykova ${ }^{2}$, T. Winkler ${ }^{3}$, G. Matziolis ${ }^{3}$, I. Stratos ${ }^{2}$, M. Schoen ${ }^{2}$, T. Bittdorf ${ }^{4}$, T. Mittlmeier ${ }^{1}$, B. Vollmar ${ }^{2}$

1 Department of Trauma \& Reconstructive Surgery, Rostock, Germany 2 Institute for Experimental Surgery, Rostock, Germany

3 Center for Musculoskeletal Surgery, Charit Berlin, Germany

4 Institute for Medical Biochemistry, Rostock, Germany

Apart from its hematopoietic effect, erythropoietin (EPO) is known as a pleiotropic cytokine which has been shown to exert anti-inflammatory and inotropic properties in cardiac ischemia/reperfusion injury. Until now, there are no studies addressing the effect of EPO on regeneration of peripheral muscle tissue following crush injury. The goal of the study was therefore to evaluate the regeneration capacity by EPO in a model of blunt skeletal muscle trauma in rats. For this purpose, a standardized open blunt crush injury was performed to the left soleus muscle of 80 male Wistar rats. Animals were treated with EPO (Reconorm $\square, 5000$ $\mathrm{IE} / \mathrm{kg}$ bw i.m.) immediately after trauma. Control rats received physiologic saline solution. For assessment of the functional recovery of muscle tissue both fast twitch and tetanic contraction capacities of the soleus muscle were measured. In addition, in vivo fluorescence microscopy of the skeletal muscle tissue microcirculation was performed on day 1 , 7,14 and 42 after trauma. Contraction force analysis revealed significantly $(p<0.05)$ higher values of relative muscle strength (given in \% to the contralateral non-injured extremity) in the EPO-treated animals when compared to saline-treated controls $(\mathrm{K})$ after 7, 14 and 42 days (fast twitch [\%] EPO-7: 53 \pm 1 ; EPO-14: 75 \pm 2 ; EPO-42: $82 \pm 4$ versus K-7:

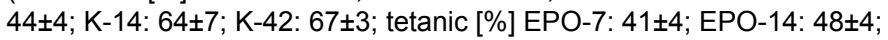
EPO-42: $79 \pm 7$ versus K-7: $30 \pm 4 ; \mathrm{K}-14: 53 \pm 4 ; \mathrm{K}-42$ : $56 \pm 6)$. In addition, EPO-treated animals presented with improved functional capillary density $(\mathrm{cm} / \mathrm{cm} 2)$ and morphological signs of angiogenesis after 1,7 and 14 days (EPO-1:284 \pm 11 ; EPO-7:321 \pm 5 ; EPO-14:291 \pm 8 versus $\mathrm{K}-1: 178 \pm 10$; $\mathrm{K}-7: 210 \pm 9 ; \mathrm{K}-14: 212 \pm 12)$. Concomitantly, trauma-induced leukocyteendothelial cell interaction and, consecutively, microvascular leakage in skeletal muscle tissue were found reduced by treatment with EPO. In conclusion, EPO results in faster and better regeneration of skeletal muscle tissue after severe trauma and goes along with improved microcirculation. Preliminary experiments provide evidence for EPO-induced local satellite cell proliferation, probably responsible for the improved muscle function upon blunt injury.

\section{1}

\section{INCORPORATION OF GROWTH FACTOR CONTAINING MATRIGEL} PROMOTES VASCULARIZATION OF POROUS PLGA SCAFFOLDS

M.W. Laschke ${ }^{1}$, M. Rücker², G. Jensen ${ }^{1}$, C. Carvalho ${ }^{3}$, R. Mülhaupt ${ }^{3}$, N.-C. Gellrich ${ }^{2}$, M.D. Menger ${ }^{1}$

1 Institute for Clin. \& Exp. Surgery, Homburg/Saar, Germany 2 Department of Oral \& Maxillofacial Surgery, Hannover, Germany 3 Freiburg Materials Research Center, Freiburg, Germany

Introduction: In tissue engineering, rapid ingrowth of new blood vessels into scaffolds is a major prerequisite for the long-term survival of three-dimensional tissue constructs. Therefore we analyzed in the present study, whether the vascularization of implanted poly-D,L-lacticco-glycolic acid (PLGA) scaffolds may be accelerated by incorporation of Matrigel.

Methods: For this purpose, we investigated in the aortic ring assay the pro-angiogenic properties of growth factor reduced Matrigel (GFRM) and growth factor containing Matrigel (GFCM). Pores of PLGA scaffolds $(\sim 3.5 \times 3.5 \times 1.5 \mathrm{~mm})$ were then filled with GFCM $(n=9)$ or GFRM $(n=8)$ before implantation into the dorsal skinfold chambers of balb/c mice. Matrigel-free scaffolds $(n=5)$ served as control. Subsequently, we analyzed vascularization, biocompatibility and incorporation of these scaffolds over 14 days by means of intravital fluorescence microscopy, histology and immunohistochemistry.

Results: In the aortic ring assay, GFCM stimulated the development of a network of tubular vessel structures with a significantly increased sprout area $\left(4.2 \pm 0.3 \mathrm{~mm}^{2}\right)$ and density $\left(335 \pm 19 \mathrm{~cm} / \mathrm{cm}^{2}\right)$ at day 6 when compared to GFRM $\left(3.2 \pm 0.1 \mathrm{~mm}^{2}\right.$ and $\left.237 \pm 17 \mathrm{~cm} / \mathrm{cm}^{2} ; \mathrm{p}<0.05\right)$. Accordingly, GFCM accelerated and improved in vivo the ingrowth of new blood vessels into scaffolds, resulting in the formation of a pericyte-coated vascular network with a significantly increased functional capillary 
density (day 14: $\left.231 \pm 8 \mathrm{~cm} / \mathrm{cm}^{2}\right)$ in comparison to the GFRM $(173 \pm 14 \mathrm{~cm} /$ $\left.\mathrm{cm}^{2} ; \mathrm{p}<0.05\right)$ and control group $\left(151 \pm 26 \mathrm{~cm} / \mathrm{cm}^{2} ; \mathrm{p}<0.05\right)$. Besides, analysis of leukocyte-endothelial cell interaction in host tissue venules located in close vicinity to the scaffolds showed no marked differences in numbers of rolling and adherent leukocytes between the observation groups, indicating that incorporation of Matrigel did not affect biocompatibility of PLGA scaffolds.

Conclusion: In our study we could show that vascularization of PLGA scaffolds is accelerated and improved by GFCM. These findings demonstrate that the combination of pro-angiogenic extracellular matrices with solid scaffold biomaterials may represent a novel approach to accelerate adequate vascularization of tissue engineering constructs.

\section{O032}

CLOSURE OF BILIARY DUCTS AND ADHESIVE STRENGTH OF FIBRINOGEN-COATED COLLAGEN PATCH AND LIQUID FIBRIN SEALANT IN AN EXPERIMENTAL LIVER RESECTION MODEL IN PIGS

D. Erdogan1, W. de Graaf², T.M. van Gulik²

1 Amsterdam, The Netherlands

2 AMC, Surgical Laboratory, Amsterdam, The Netherlands

Background \& aims: Surgical management of the cut surface after liver resection consists of hemostasis and closure of bile leaks. Several fibrin sealants may be used to attain this goal. No studies have assessed the efficacy of these sealants for prevention of biliary leaks, nor the adhesive strength of these sealants to the resection surface of the liver. The aim of this study was to assess the efficacy of sealing of biliary ducts and the adhesive strength of two types of commercially available sealing agents, i.e. a fibrinogen-coated collagen patch (TachoSil $($ ) ) and

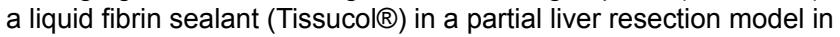
pigs.

Materials and methods: Fibrin sealant is a hemostatic agent composed of fibrinogen and thrombin. The fibrinogen-coated collagen patch Tachosil囚 (Nycomed GmbH, Breda, Netherlands) was compared with the liquid fibrin sealant Tissucol® (Duo 500, 2 ml, Baxter Hyland Immuno, Uden, Netherlands). Eight pigs (Vendrig, Amsterdam, The Netherlands) weighing $35-45 \mathrm{~kg}$ were randomized in 2 groups. Each pig underwent laparotomy and resection of the left medial lobe of the liver. Larger blood vessels in the resection surface were suture closed, whereas small bile ducts were left unclosed. Thereafter, the tip of a 16 Ch silicon catheter was introduced into the common bile duct through a distal incision until the confluence of the hepatic ducts. Pigs were assigned to two groups: application of Tachosil $(n=4)$ or Tissucol $(n=4)$ on the resection surface of the remnant liver. After 2 hours, pressure in the intrabiliary system was increased gradually by infusing sodium chloride through the catheter. Results are expressed as mean \pm standard error of the mean (SEM). Student's t-test was used for differences between groups. $P<0.05$ was considered significant.

Results: No difference was seen in the number of blood vessels closed in the resection surface of the group with Tachosilß compared to the group with Tissucol $R(4.0 \pm 0.4$ vs. $5.5 \pm 0.5 ; P=0.0677)$. No difference was observed in the number of bile ducts in the resection surface of the group with Tachosil $(\mathrm{R}$ compared to Tissucol $(\mathrm{R})(2.5 \pm 0.3$ vs. $2.3 \pm 0.3$; $P=0.5416)$. No bleeding or bile leakage was observed in both groups after application of the sealants on the resection surface of the liver remnant. No significant difference in haemostasis time after Tachosil $(R$ or Tissucol was observed $(210.0 \pm 12.9 \mathrm{sec}$ vs. $208.8 \pm 14.2 ; \mathrm{P}=0.9506)$. The pressure that could be resisted with Tissucol $R$ was significantly lower compared to that after application of TachoSil $\mathbb{R}(77.0 \pm 8.6 \mathrm{mmHg}$ vs. $131.8 \pm 16.3 ; \mathrm{P}=0.0405$ ).

Conclusion: Application of TachoSil $R$ on the resection surface is equally effective in obtaining haemostasis and in sealing of biliary ducts

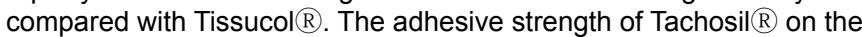
resection surface, however, is superior to Tissucol $R$.

\section{O033}

\section{IMPROVEMENT OF POST-INFARCTION CARDIAC REMODELING BY GRAFTING STROMAL STEM CELLS WITH A HYALURONAN- BASED SCAFFOLD}

\section{A. Tsivian}

University Of Bologna, Bologna, Italy

Aim of study:Tissue engineering has been proposed as a strategy to regenerate the infarcted myocardium. In this study we aimed to assess the effects of stromal stem cells, delivered into the infarcted area on a hyaluronan-based scaffold, on the remodeling of the infarcted myocardium in rats.

Materials and methods: Donor animal hearts were harvested, subjected to left descending coronary artery occlusion, and then grafted onto the abdominal aorta of receiving syngeneic rats by means of a terminolateral aorto-aortic anastomosis. After 2 weeks, a $3 \times 3 \mathrm{~mm}$ pouch was made in the thickness of the ventricular wall at the level of the postinfarction scar. A hyaluronan-based scaffold, previously engineered for 3 weeks with rat bone marrow mesenchymal stem cells (MSCs) was introduced into the pouch and the myocardial edges sutured with some 8-0 stitches.

Results: Two weeks later we observed that the hyaluronan fibres had not been substantially degraded and most MSCs had migrated to the surrounding infarcted area where they were especially found close to small-sized vessels. Scar tissue was moderate in the engrafted region and the thickness of the corresponding ventricular wall was comparable to that of the non-infarcted remote area. Also, the left ventricular shortening fraction, evaluated by M-Mode echography, was not found decreased when compared to that measured just before construct transplantation.

Conclusion: The outcome of this study suggests that post-infarction myocardial remodeling can be favourably affected by the grafting of MSCs delivered through a hyaluronan-based scaffold.

\section{O034}

\section{DOES PEAK WALL STRESS CORRELATE BETTER WITH ABDOMI-} NAL AORTIC ANEURYSM EXPANSION THAN AORTIC DIAMETER?

\section{M.N.A. Rahman, S. Gulati, A. Mekako, P.T. McCollum, I.C. Chetter} Academic Vascular Surgical Unit, HULL, United Kingdom

Introduction: Level 1 evidence clearly supports ultrasound surveillance in abdominal aortic aneurysm (AAA) $<5.5 \mathrm{~cm}$ in maximum AP diameter, with scanning interval set according to the size of aneurysm. Larger aneurysms tend to expand at a greater rate than smaller aneurysms. However, AAA expansion is variable and difficult to predict, and size may not always predict expansion. We aimed to assess whether peak wall stress (PWS) measured through finite element analysis, correlates better with AAA expansion.

Methods: 38 patients, 31 men, median age of 76.5 (range 54-93) years, currently under AAA surveillance were studied. All patients underwent abdominal CT scan to characterise the aneurysms and measure PWS through finite element analysis. Expansion rates were derived from sequential ultrasound scans. Correlation between PWS and expansion rate, as well as between size and expansion rate was analysed using Spearman Test (SPSS for Windows v14).

Results: Mean aortic diameter was $4.68+/-0.74 \mathrm{~cm}$ and mean PWS was $0.41+/-0.14 \mathrm{MPa}$. Spearman rank order correlation coefficient ( $\mathrm{rs}$ ) of PWS and AAA expansion rate was $0.28(p=0.08)$. Spearman rank order correlation coefficient ( $r s$ ) of aorta AP diameter and AAA expansion rate was $0.17(p=0.30)$.

Conclusion: In this cohort of patients the correlation between PWS and AAA expansion rate was not significant however the correlation was superior to that between AAA diameter and expansion rate, and this deserves further investigation.

\section{5}

\section{INFLUENCE OF ERYTHROPOIETIN ON HEALING OF COLONIC} ANASTOMOSIS AFTER CHEMOTHERAPY IN RATS

T. Kucukkartallar, A. Tekin, F. Aksoy, C. Vatansev, M. Sahin, M. Belviranli Selcuk University Meram Medical Faculty, Konya, Turkey

Aim: We aimed to search the effects of erythropoietin on anastamos healing in rats to which chemotherapy was applied and intestine anastomosis was made.

Material and methods: The study was carried out at Selcuk University Experimental Research Center (SUDAM). The rats were divided into 4 groups including 10 subjects. The subjects in all groups were weighed before the surgical operation and a midline laparotomy was made and then, sigmoid colon transsected and a one layer anostomosis was made. Nothing but only standard feeding was given to the subjects in the 1 st group after making colonic anastomosis. Subcutan erythropoietin 
injection was administered to the 2nd group after colonic anastomosis The 3rd group was injected intraperitoneal 5- fluorouracil after colonic anastomosis. The 4th group was given intraperitoneal 5- fluorouracil after colonic anastomos, and subcutan erythropoietin injection was administered. On the 7 th day following the anastomosis, the rats were weighed and they were sacrificed. Anastomosis bursting pressure among the groups, hydroxyproline levels in the tissue samples taken from anastomosis line and collagen and rich bind tissue formation from the fibroblasts histopathological, the rise angiogenesis, intensity in inflammation and the existence of necrosis were evaluated

Findings: In the rats to which erythropoietin was applied, the bursting pressures and hydroxyproline levels were significantly higher than those of the other groups statistically. During the histopathological examination, it was seen that rich bind tissue formation from angiogenesis and collagen in the erythropoietin group rose while there was a significant reduction in the formation of inflammation and necrosis. These positive effects of erythropoietin was also established in the rats to which 5- fluorouracil was applied.

Conclusion: In the rats to which colo-colic anastomosis with intraperitoneal 5-FU application, it was considered that erythropoietin had positive effects on angiogenesis with its influences on proliferation and restructuring of wound healing. Thus, it can be expected that erythropoietin can reduce the risk of anastomotic leakage after colo-colic anastomosis and in conditions such as chemoteraphy, which negatively affects the recovery of anastomosis.

\section{O036}

\section{RECEIVING AN ORAL CARBOHYDRATE-RICH DRINK PRIOR TO SURGERY: COMPARISON OF METABOLIC RESPONSES BETWEEN PATIENTS WITH AND WITHOUT INSULIN RESISTANCE}

M. F. Can, G. Yagci, S. Gorgulu, A. Simsek, G. Demirel, T. Tufan Gulhane School of Medicine, Ankara, Turkey

Background and aim: Preoperative use of carbohydrate-rich drinks has been proposed to reduce fasting induced insulin resistance (IR) and to ameliorate related metabolic malfunctions. However, these positive effects and reliability of the application are unclear for those cases who have already an IR in preoperative period. We investigated in this prospective controlled study the metabolic responses to the intake of carbohydrate-rich drink in patients with IR undergoing surgery.

Methods: Non-diabetic thirty-four patients scheduled for cholecystectomy or thyroidectomy constituted the study group with permission of loca ethics committee. All cases ingested a special carbohydrate-containing

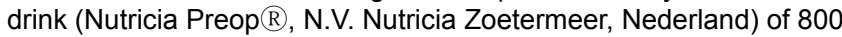
$\mathrm{ml}$ evening before the operation and $400 \mathrm{ml}$ two hours before beginning the surgery (morning dose). Blood samples for plasma glucose, plasma cortisol and serum insulin levels were taken immediately before morning dose (I), 40th (II) and 90th (III) minutes after intake of the drink, and also at the time of anesthesia induction (IV). Homeostasis Model Assessment II (HOMA-II) scores were calculated by using the values of fasting glucose and insulin levels and used to identify the IR patients. The patients who have HOMA-II score $>2.5$ were considered as IR. The differences between measurements of IR and non-IR (NIR) groups were analyzed using Mann-Whitney-U test and the results were given as mean \pm SEM.

Results: Eight of thirty-four patients were IR while remaining twenty-six were NIR with HOMA-II scores $2.9 \pm 0.8$ and $1.79 \pm 0.9$, respectively. The median age was 50 (25-70) in IR group and 51 (21-76) in NIR group. There were no statistically significant differences between two groups for male/female ratio and body-mass index $(0.72$ ' $28.8 \pm 1.97$ and 0.84 ' $26.2 \pm 0.87$, respectively). Although the mean plasma glucose levels measured from samples obtained at all four measurement points for IR group were higher, the differences did not reach to significance ( $p$ values: $0.092,0.372,0.792$ and 0.088 for I, II,III and IV, respectively). The cortisol levels were also similar in both groups ( $p$ values: 0.808 , $0.935,0.839$ and 0.310 with the same rank). The serum insulin levels were elevated in IR group initially, but then gradually decreased to the corresponding levels which were observed in NIR patients.

Conclusion: During the preparation to the scheduled surgery, insulin resistant patients receiving carbohydrate-rich drink appear not to be affected by the adverse metabolic effects of the beverage. Furthermore, it may be possible for patients with IR also to obtain the probable beneficial effects referred to these drinks. We suppose that they can undergo to elective surgery safely, like non-insulin resistant cases.
O037

IS THERE A PROGNOSTIC ROLE OF NUCLEAR FACTOR KAPPA B IN PERIPHERAL MONONUCLEAR CELLS DURING ACUTE NECROTIZING PANCREATITIS IN RATS?

\author{
E. Alhan ${ }^{1}$, S.T. Turkyilmaz ${ }^{1}$, S.T. Topaloglu², C.E. Cengiz ${ }^{2}$, B.V.K. Vanizor \\ Kural $^{2}$, D.F. Flinte ${ }^{2}$ \\ 1 Karadeniz Technical University, Trabzon, Turkey
}

The aim of this study was to investigate the prognostic role of nuclear factor kappa $B$ (NFKB) in peripheral mononuclear cells (PMNC) during acute necrotizing pancreatitis (ANP) in rats. ANP was induced by an intravenous infusion of cerulein $5 \mu \mathrm{g} / \mathrm{kg} /$ hour over six hours superimposed on a standard infusion of $1.2 \mathrm{ml} / \mathrm{kg}$ glycodeoxycholic acid $10 \mathrm{mmol} / \mathrm{L}$ into the biliary-pancreatic duct for 10 minutes at $30 \mathrm{~mm} \mathrm{Hg}$. The rats were divided in five groups. The first group was selected control group. ANP was induced in the remainder groups and the groups were followed up for $6 \mathrm{~h}, 12 \mathrm{~h}, 24 \mathrm{~h}$ and $48 \mathrm{~h}$ in groups $2,3,4$ and 5 respectively. Research parameters are mortality rate, serum amylase, urea, alanine transferase (ALT), pancreatic histology and the activity of NFKB. The activity of NFKB in PMNC is measured as two subunits of NFKB, p50a and p65a. The induction of ANP resulted in significant increase in mortality rate, pancreatic necrosis, and serum activity of amylase, ALT, urea and the activity of p65a. The activity of p50a didn't show any significant change after the induction of ANP. There is significant correlation between the mortality rate and pancreatic necrosis according to the time-point. But there was no correlation between p65a levels of NFKB and the mortality rate. The measurement of $\mathrm{p} 65$ a level of NFKB in PMNC has no prognostic role during ANP in rats. It has limited value in the treatment of ANP

\section{O038}

\section{THE EFFECTS OF PARENTERAL GLUTAMINE ADMINISTRATION ON INTESTINAL ADAPTATION AND ANASTOMOTIC HEALING IN A RAT MODEL OF SHORT BOWEL SYNDROME}

D. Ucar, H. Kocdor, A.E. Canda, S.M. Unlu, R. Cehreli, M.A. Kocdor Dokuz Eylul University, IZMIR, Turkey

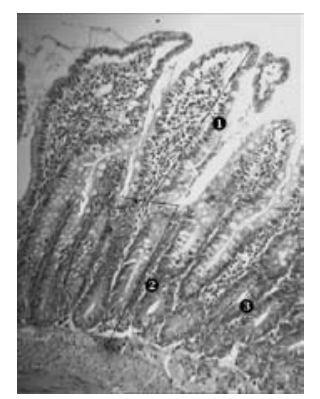

Histological appearance of the ileum (H\&E stain)

Background \& aims: Short bowel syndrome (SBS) is a clinical condition characterized by malabsorbtion of nutrients and fluids which occurs after major intestinal resection. An adaptation process begins immediately after resection in order to increase the mucosal surface and absorption however, this process is commonly not sufficient. Intestinal adaptive factors may provide good alternative for SBS treatment. The efficacy and benefits of glutamine on adaptation process after massive intestinal resection is not clear. The aim of the study was to determine the effects of parenteral glutamine administration on intestinal adaptation in a rat model of SBS.

Methods: Fourteen male Wistar rats were divided into two groups and all were underwent $75 \%$ small bowel resection. Within each group, rats were assigned to 14 days of treatment with subcutaneous glutamine $(0.3 \mathrm{~g} / \mathrm{kg} / \mathrm{day})$ or saline daily. Intestinal adaptation parameters (total number of goblet cells, villus heights, crypt depths, and mucosal thickness) as well as the anastomotic healing parameters including collagen contents were measured.

Results: No significant difference was detected in terms of total number of goblet cells, villus heights, crypt depths, mucosal thickness, and the amount of anastomotic newly synthesized collagen between the two groups.

Conclusion: Parenteral glutamine administration is not effective on the intestinal adaptation process and anastomotic healing in a rat model of SBS. 
0039

DOES INTERLEUKIN 8 PLAYS A ROLE IN GROWTH AND METASTASIS OF PANCREATIC ADENOCARCINOMA.

F.H. Syed, M. El-Bahrawy, E. Shawish

Imperial college London, Prescot, United Kingdom

Introduction: IL-8 is a CXC chemokine that plays an important role in acute inflammation, angiogenesis, tumour growth and metastasis through its two receptors IL-8RA and IL-8RB. Recent studies have shown that IL-8 and IL-8 receptors play an important role in growth and metastasis of pancreatic adenocarcinoma (PACa). The aim of our study was to examine the expression of IL8 and IL8 receptors on PACa in relation to the grade and stage of tumour.

Materials and methods: We examined the expression of IL-8 and IL-8 receptors in 52 surgically resected specimens of PACas. Tumour blocks of different tumour grades were selected. Immunohistochemical analysis was performed by using indirect horseradish peroxidase-labelled streptavidin-biotin method. The presence and intensity of staining was scored and correlated with tumour grade and stage.

Results: IL-8 was expressed in $13 / 52(25 \%)$ cases of PACa. The inflammatory cells within the tumour stroma strongly expressed IL-8.

The expression of IL-8RA was detected in 75\% (39/52) and IL-8RB was expressed in $79 \%(41 / 52)$ of PACa. The expression of all three antigens was mainly cytoplasmic. In PACa, 7/52 tumours co-expressed all 3 antigens while 32 tumours co-expressed both receptors.

Conclusion: The results show that IL-8, IL-8RA and IL-8 RB were over expressed in PACa, which suggests a role of IL-8 and its receptor in the growth and progression of PACa. The simultaneous presence of both IL-8 and IL-8 receptors suggests an autocrine signalling system.

\section{0}

\section{EFFECTS OF PREOPERATIVE BENEFIBER ON COLONIC ANASTOMOTIC HEALING IN A RAT MODEL OF PREOPERATIVE RADIOTHERAPY}

A. Sevinc, B. Aydogan, A.E. Canda, C. Terzi, G. Oktay, O. Cetinkaya, M. Fuzun

Dokuz Eylul University, IZMIR, Turkey, Manisa State Hospital Turkey

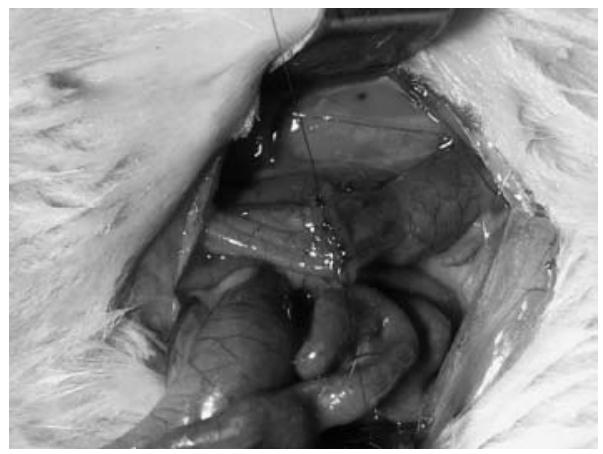

Colonic anastomosis

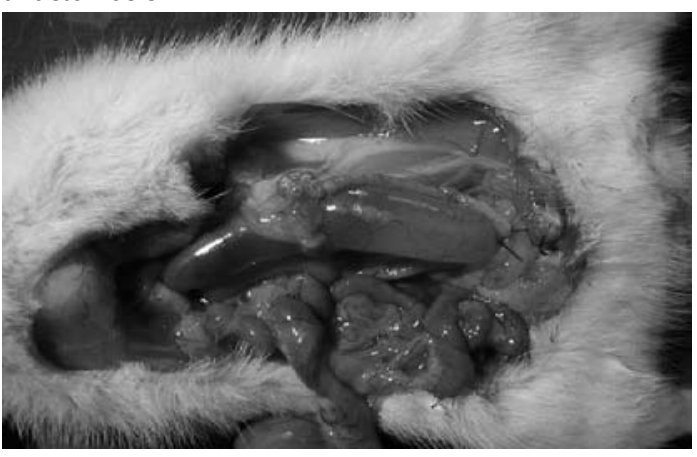

Anastomotic burst pressure measurement

Background and aims: Preoperative radiotherapy interferes with anastomotic healing in patients with rectal cancer. We investigated the effects of preoperative oral Benefiber $R$ on irradiated colonic anastomosis in a rat model of preoperative radiotherapy.
Methods: Forty male Wistar rats were divided into four groups and all rats underwent $1 \mathrm{~cm}$ left colon resection and primary anastomosis: Group I (Control group, n=10) no additional treatment; Group II (Benefiber pretreatment group, $n=10$ ) had 5 days Benefiber by orogastric route preoperatively; Group III (Preoperative radiotherapy group, $n=10$ ) were irradiated for 4 and 8 days preoperatively; Group IV (Preoperative radiotherapy and Benefiber pretreatment group, $n=10$ ) were irradiated for 4 and 8 days preoperatively and had 5 days Benefiber by orogastric route after the second day of irradiation. Abdominal wound healing, intraabdominal adhesions, anastomotic complications, anastomotic burst pressures and sites were recorded. Histopathologic evaluation and the biochemical collagen analyses were performed for evaluation of anastomotic healing.

Results: No significant difference was observed between groups in for abdominal wound healing, intraabdominal adhesion scores, and anastomotic complications. Anastomotic burst pressures were significantly lower in group III ( $P=0.032)$; which was significantly higher in group II $(P=0.016)$. Salt soluble collagen concentrations were significantly higher in group II than group III $(P=0.008)$. Total and salt soluble collagen concentrations were significantly higher in group IV than group III ( $\mathrm{P}=0.016)$. On histopathological evaluation necrose was prominent in group III than group IV $(P<0.01)$, granulocyte count was significantly higher in group II and group IV compared to group III $(P<0.01)$. Fibroblast count was significantly lower in group III than the other groups $(P<0.01)$. Conclusion: These findings suggest beneficial effects of Benefiber on anastomotic healing in a rat model of preoperative radiotherapy and colonic anastomosis

\section{O041}

RECOMBINANT HUMAN ERYTHROPOIETIN ALPHA MODULATES THE EFFECTS OF RADIOTHERAPY ON COLORECTAL CANCER MICROVESSELS

W.P. Ceelen, N. Boterberg, N. Smeets, N. Van Damme, N. Peeters, N. Pattyn

University Hospital, Ghent, Belgium

Purpose: to study modulation of radiotherapy (RT) effects on CC531 colorectal cancer microvessels by administration of recombinant human erythropoietin (rhEPO) in a WAG/Rij rat model.

Experimental Design: tumor bearing rats were administered $3 \times 0.1 \mathrm{ml}$ of rhEPO weekly. Five days before and after $5 \times 5$ Gy of RT, dynamic contrast enhanced (DCE) MRI was performed after rapid iv injection of 792 , a macromolecular MR contrast agent. Mathematical modelling resulted in parametric maps of endothelial permeability surface product (PS), plasma flow (F), and blood volume (V). Imaging was combined with pO2 and laser doppler flow (LDF) measurements. After the second set of measurements, tumors were analysed for microvessel density (MVD), diameter and fractal dimension (MFD). Expression of VEGF, HIF-1 $\alpha$, $\mathrm{Bax}$, and $\mathrm{Bcl}-2$ was determined immunohistochemically.

Results: RT significantly reduced PS and $V$ in control rats, but not in rhEPO treated rats, while $F$ was unaffected by RT in both groups. Oxygenation was significantly better in rhEPO treated animals, and RT induced a heterogeneous reoxygenation in both groups. LDF was significantly lower following RT in the central tumor region of control rats, while no changes in LDF were seen in rhEPO treated rats. Microvessel diameter was significantly larger in rhEPO animals, while MFD was lower in the tumor core. VEGF expression was significantly lower in the

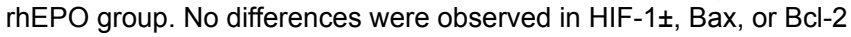
expression.

Conclusion: rhEPO results in spatially heterogeneous modulation of RT effects on tumor microvessels. Direct effects of rhEPO on neoplastic endothelium are likely to explain these findings in addition to indirect effects induced by increased oxygenation.

\section{O043}

\section{PANCREATIC TRAUMA CASE SENSES WITH REVIEW OF}

\section{LITERATURE}

A. Supe ${ }^{1}$, C.V. Kantharia ${ }^{2}$

1 Mumbai, India

2 Seth G S medical College, Mumbai, India

Introduction: Pancreatic trauma though uncommon is associated with high morbidity and mortality. Diagnosis is often difficult and surgery 
poses a formidable challenge. This study analyse a single institutional experience of management of Pancreatic trauma.

Study design: 17 patients of Pancreatic trauma were identified from our database over the last five years from the year 2000-2005. they were analyzed with respect to the mode of injury, diagnostic modalities, associated injuries, grade of pancreatic trauma and their management. As per the Modified Lucas grading for Pancreatic Trauma , 2 patients had Grade I injury, 3 patients had Grade II injury, 5 patients had grade III injury and 7 patients had grade Iv injury.

Immediate diagnosis of pancreatic trauma could be reached only in 3 patients. CT scan was the main diagnostic modality. 7 patients underwent distal pancreatectomy with splenectomy, patients underwent Pylorus preserving pancreato-duodenectomy, 3 patients were subjected to debridement with external drainage and 2 patients of Grade I were treated conservatively. Complications in form of pancreatic fistula, pancreatitis and pseudo-cyst formation was seen in $3(17.05 \%), 2(11.7 \%)$ and $1(5.4 \%)$ patients respectively. Mortality was in $4(23.52 \%), 2$ of which had vascular injury involving the superior mesenteric vessels, and 2 who had undergone Pancreato-duodenectomy.

Conclusion: We conclude that blunt abdominal trauma is the common mode of sustaining pancreatic trauma. It is usually associated with bowel or vascular injury. CT scan is the most sensitive modality of investigation for diagnosis. Appropriate management comprising of accurate assessment of injury, based on Lucas grade, adherence of basic concept of management of vascular management, judicious resection and adequate drainage is the key to successful outcome. Morbidity and mortality in pancreatic trauma though significant is primarily due to associated injuries.

\section{O044}

RADIOIMMUNOTHERAPY AND HYPERTHERMIC INTRAPERITONEAL CHEMOTHERAPY AS ADJUVANT THERAPIES AFTER CYTOREDUCTIVE SURGERY FOR EXPERIMENTAL PERITONEAL CARCINOMATOSIS: EFFECTS ON ANASTOMOTIC HEALING IN THE INTESTINE AND REPAIR OF THE ABDOMINAL WALL

F. Aarts, R.P. Bleichrodt, O.C. Boerman, T. Hendriks UMCN St. Radboud Nijmegen, Nijmegen, The Netherlands

Background and aim: Extensive surgical debulking procedures (cytoreductive surgery or $\mathrm{CS}$ ) in combination with hyperthermic intraperitoneal chemotherapy (HIPEC) are considered as today's standard of care for peritoneal carcinomatosis (PC). Despite a limited gain in survival, these procedures result in substantial morbidity and mortality rates of up to $50 \%$ and $8 \%$, respectively. Since bowel anastomoses are almost invariably constructed during the operation, a substantial proportion of its morbidity is due to anastomosis-related complications such as anastomotic dehiscence, with or without concomitant intra-abdominal abscess formation. Previously, we have demonstrated the therapeutic effect of radioimmunotherapy (RIT) as an adjuvant to CS in a preclinical model of PC. The purpose of the present experiment is to compare the effects of RIT and HIPEC on the healing of both bowel anastomoses and wounds in the abdominal wall.

Methods: PC was induced by intraperitoneal inoculation of CC-531 colon carcinoma cells in Wag/Rij rats. Animals were subjected to CS only, CS+RIT or CS+HIPEC. The adjuvant treatments were applied immediately after $\mathrm{CS}$ and anastomotic construction. The latter consisted of resection $(1 \mathrm{~cm})$ and anastomosis, by 8 interrupted inverting sutures, of both terminal ileum and distal colon. RIT consisted of $74 \mathrm{MBq}$ 177lutetium-labelled anti-CC531 antibody MG1. HIPEC was performed by a closed abdomen perfusion technique using mitomycin-C (MMC), $16 \mathrm{mg} /$ I, during 60 minutes. Anastomotic and abdominal wall strength strength were measured by means of breaking strength and bursting pressure at 3 and 5 days after surgery.

Results: The median intra-abdominal temperature during the HIPEC procedure, measured at the site where the greater omentum was removed, was $41.0^{\circ} \mathrm{C}$. Anastomotic breaking strength measured at day 3 and 5 was similar in all groups, both in ileum and colon. At day 5 , bursting pressure in ileum and colon anastomoses was lowest in the CS+HIPEC group, with $P<0.05$ and $P<0.001$ vs the CS group, respectively. In addition, in the CS group, anastomotic failure was outside the anastomosis in 6/12 animals whereas in the CS+HIPEC and CS+RIT group, anastomotic failure was outside the anastomosis in 1/12 and $4 / 12$ animals, respectively. Abdominal wall strength was highest in the CS group at both day 3 and 5 after surgery, (CS vs. CS+HIPEC $P<0.05$ at day 3 and CS vs. HIPEC $P<0.001$ at day 5). In addition, there was a significant decreased abdominal wall strength after CS+HIPEC when compared to CS+RIT: $P<0.05$ at day 5 .

Conclusion: Anastomotic and abdominal wall wound strength after cytoreductive surgery is decreased when followed by the adjuvant application of HIPEC. In contrast, adjuvant RIT did not decrease wound strength. Adjuvant RIT should therefore be considered as an alternative adjuvant treatment after cytoreductive surgery of $\mathrm{PC}$ of colorectal origin in the clinical setting.

\section{O045}

SHORT-STAY HOSPITALIZATION IN THYROID SURGERY. PROSPECTIVE RANDOMIZED TRIAL IN 200 PATIENT OPERATED FOR BENIGN MULTINODULAR GOITER

F. Fama ${ }^{1}$, O. Saint-Marc ${ }^{2}$, A. Piquard², A. Cogliandolo ${ }^{3}$, R. Pidoto ${ }^{3}$, M.A. Gioffre' Florio ${ }^{3}$

1 Policlinico Universitario Messina, Messina, Italy

2 Centre Hospitalier Regional, Orleans, France

3 Policlinico Universitario, Messina, Italy
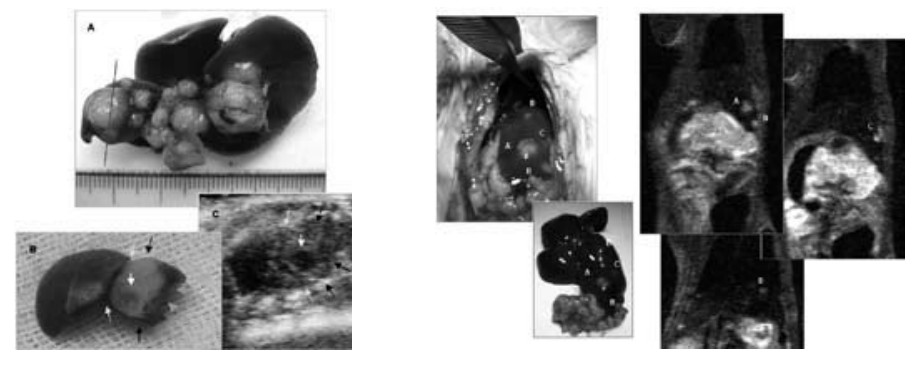

Introduction: Total thyroidectomy represents the gold standard for treatment of thyroid cancer and at present for all benign multinodular goiter. Recently short-stay hospitalization in thyroid surgery was emphasized in order to reduce costs. A prospective and randomized trial was performed to evaluate complication rates in two groups, who received 2 different surgical techniques. Particular care was taken in studying the incidence of post-operative haematomas as a potentially fatal damage of the airway system.

Patients and methods: During 11 months 200 elective total thyroidectomies for benign multinodular goiters were performed in 164 women (mean age of 52 years) and 36 men (mean age of 47 years). All patients were admitted. One hundred (100) were treated by traditional technique 'clamp-and-tie' and 100 cases by a LigaSureTM device. All patients were assessed by routine blood test, hormone levels, TSH and thyroglobulin dosage, cervical ultrasound and in 18 cases by thyroid scintigraphy. Preoperative, postoperative ( 24 hours and 6 weeks) calcium homeostasis was evaluated by serum calcium levels. Before surgery, cardio-thoracic status and vocal cords functionality were evaluated by anaesthetists and otorhinolaryngology (indirect laryngoscopy). Patients with a suspect of malignancy were excluded.

During surgery external and recurrent laryngeal nerves and all 4 parathyroid glands were identified in nearly all cases.

Accurate haemostasis of the para-tracheal space and subcutaneous layer was performed and only in 16 cases a little aspirate drainage was positioned. Clips or surgical glue were used to close surgical wounds. In addition, all patients were prescribed the indicated weight-related thyroxine dosage. Of 200 patients, 185 were discharged the day after surgery $(92,5 \%), 14$ patients within $48 \mathrm{~h}(7 \%)$ and only one-case $(0,5 \%)$ at 4 th day. All patients were advised to watch out for signs and symptoms of hypocalcaemia and respiratory compression and had a 24 h emergency contact number.

Results: In our study, post-operative complications were observed in 70 patients (35\%) including transitory and permanent lesions. In detail: 42 (21\%) hypocalcemia (3 permanent metabolic disorder), 23 (12\%) injury of recurrent nerve (2 laryngeal paralysis) and $4(2 \%)$ cervical haematomas ( 3 cases required urgent reoperation). During thyroidectomies, 78 parathyroid auto-transplantation was necessary. Transitory hypocalcemia (39 patients, 19,5\%) was treated with oral calcium supplementation sometimes associated to Vitamin D3 and restored (eucalcemia) at the follow-up ( 6 weeks). No mortality was observed. Mean operative time was significantly shorter, about seven minutes, using LigaSureTM device.. We discharged $185(92,5 \%)$ patients one day after surgery. In 14 $(7 \%)$ patients (10 transitory hypocalcemia, 3 haematomas and 1 dysphonia) and in one-case $(0,5 \%)$ with severe hypocalcemia the discharge was, respectively, at 2 nd and at 4 th post-operative day. 
Discussion: Short-stay hospitalization could become the gold standard in thyroid surgery. Our complication rate was in accordance with literature rates. However we suggest a careful surveillance for at least 24 hours (1 night) avoiding potentially lethal complications. In our experience 3 bleeding complications occurred in the 1st post-operative night as a real surgical emergency; they needed a short stay in resuscitation for a prolonged respiratory shock with hypercapnia in two cases.

\section{O046}

PROGNOSTIC SIGNIFICANCE OF PATHOLOGICAL RESPONSE TO NEOADJUVANT CHEMORADIATION IN CARCINOMA OF THE ESOPHAGUS AND GASTRIC CARDIA

W.P. Ceelen, N. Fierens, N. Van De Putte, N. Boterberg, N. Peeters, N. Van Damme, N. Varin, N. Pattyn

University Hospital Ghent, Ghent, Belgium

Background: Neoadjuvant chemoradiation (CRT) is commonly used in locally advanced esophageal cancer. We analyzed the prognostic significance of pathological response in a cohort of patients treated with CRT followed by surgery.

Methods: Patients with cT4NOMO and cT3-4N1M0 disease received neoadjuvant CRT followed by Ivor Lewis esophagectomy with two field lymphadenectomy. Chemotherapy consisted of cisplatin $(80 \mathrm{mg} / \mathrm{m} 2)$ plus fluorouracil $(800 \mathrm{mg} / \mathrm{m} 2)$ on days 1 and 22 . Radiotherapy was given in 20 fractions of 180 cGy. Actuarial overall survival (OS) was calculated using the Kaplan Meier method. Data represent median $\pm 95 \% \mathrm{Cl}$. Results: 77 patients were treated from 5/2003 until 8/2006. Clinically and iconographically, all patients were stage III with $59.7 \%$ node positive. Histologically, $88.7 \%$ were adenocarcinomas. In hospital mortality was $5.3 \%$, and major morbidity occurred in $36 \%$ of patients. Pathological stage distribution was as follows: pathological complete response (pCR), 22.7\%; stage I, 10.7\%; stage IIA, 24\%; stage IIB, 10.7\%; stage III, $24 \%$; stage IV, $1.3 \%$, and ypTON1, $6.6 \%$. Downstaging of either T or $\mathrm{N}$ stage was observed in $67.5 \%$ of patients resulting in an OS of 46 months $(9.5-82.5)$ while OS was 17 months (2.6-31.4) in patients without pathological response $(\mathrm{P}<0.01)$. In patients with a pCR, OS was 63.2 months (38-88).

Conclusion: neoadjuvant CRT results in a substantial $\mathrm{pCR}$ rate in esophageal cancer. Response to CRT is associated with improved survival compared to non responders. Further study will have to determine whether this finding reflects a difference in tumor biology or a real effect of CRT.

\section{7}

OUTCOME OF PERITONEAL CARCINOMATOSIS TREATED WITH A MULTIMODAL APPROACH: PREDICTORS OF LONG TERM SURVIVAL IN A COHORT OF 130 PATIENTS

W.P Ceelen, N. Fierens, N. Peeters, N. Pattyn

University Hospital Ghent, Ghent, Belgium

Background: Cytoreductive surgery with hyperthermic intraperitoneal chemoperfusion (HIPEC) has been introduced in the management of peritoneal carcinomatosis (PC) with biologically favorable features. We analyzed factors predictive of long term overall survival (OS) following this intensive therapy.

Methods: In an eight year period, 130 patients were treated $(53 \%$ female, mean age $56 \pm 1$ years). The origin of $P C$ was colorectal cancer (CRC, 58\%), pseudomyxoma peritonei (PMP, 9\%), ovarian cancer $(17 \%)$, primary peritoneal mesothelioma $(10 \%)$, and gastric cancer (6\%). Surgery was followed by HIPEC with mitomycin C, oxaliplatin or cisplatin depending on tumor histology and previous chemotherapy. Actuarial survival was calculated using the Kaplan Meier method and related to clinicopathological variables using univariate and multivariate regression analyses.

Results: Postoperative 30 day mortality was $1.5 \%$ while major morbidity developed in $18 \%$ of patients. Median OS (months) was as follows: CRC, 20; PMP, 38; ovarian cancer, 19; gastric cancer, 10; and mesothelioma, 9. Macroscopically complete resection was achieved in $41 \%$ of patients resulting in a median OS of 40 months. Primary tumor histology, presence of ascites, completeness of cytoreduction (CC), and adjuvant chemotherapy were significantly related to $O S$ in univariate analysis. The extent of disease score before surgery $(p=0.29)$ was not related to OS. In a multivariate proportional hazards model, OS was significantly related to the presence of acites $(P=.015)$, tumor histology $(P=.029)$, and adjuvant chemotherapy $(\mathrm{P}<.001)$, but not to $\mathrm{CC}$.

Conclusion: Long term survival following cytoreduction with HIPEC is associated with tumor histology, absence of ascites, and adjuvant chemotherapy.

\section{O048}

NEW T-SHAPED POLYPROPYLENE/ePTFE COMPOSITE PROSTHESIS DESIGNED TO STRENGTHEN A MIDLINE LAPAROTOMY CLOSURE

J.M. Bellon, G. Pascual, M. Rodriguez, N. Garca-Honduvilla, P. LopezHervas, J. Buján

Alcala University, Alcala De Henares, Spain,

Introduction: Despite numerous experimental and clinical studies designed to test different suture materials and closure techniques, the incidence of incisional hernia is still high, especially following a midline laparotomy closure. In this study, we propose a new closure method in which a prosthetic material is included between the two cut edges of the surgical wound.

Materials and methods: The prosthesis was a strip of polypropylene (PP) plus expanded polytetrafluoroethylene (ePTFE), T-shaped in cross-section. This strip was used to close a $7 \mathrm{~cm}$-long midline incision made $3 \mathrm{~cm}$ from the xiphoid process in 63 New Zealand White rabbits, by sandwiching the vertical arm of the T-mesh ( $T$ placed upside down) between the surgical wound edges. The horizontal arm, coated with ePTFE, made contact with the visceral peritoneum. The T-mesh was fixed by a single plane PP $3 / 0$ running suture through the wound edges and vertical arm of the inverted T. Animals were either not subjected to laparotomy (control group, $\mathrm{n}=15$ ) or underwent a midline laparotomy that was then closed by a simple PP suture (group I, $\mathrm{n}=24$ ) or by including the inverted T-mesh between the laparotomy edges (group II, $\mathrm{n}=24$ ). At the time points 3 weeks, 6 weeks and 6 months post surgery, morphological (LM and SEM), immunohistochemical (RAM-11) and biomechanical (INSTRON 3340 tensiometer) analyses were performed. The Mann Whitney U-test was used to compare data among the study groups. Surgery was always performed by the same surgeon.

Results: The T-mesh induced the increased thickness and width of the scar at the linea alba. Neoformed host tissue appeared around the PP mesh filaments. Macrophage numbers diminished from 3 weeks to 6 months post surgery. Between weeks 3 and 6 , the tensile strength of the surgical wound incorporating the T-mesh increased to exceed the strength of the wound closed by simple suture ( 6 weeks: $43.99 \pm 4.17 \mathrm{~N}$ vs $59.96 \pm 10.94 \mathrm{~N}, p<0.05)$. At 6 months, the resistance of the T-mesh suture was even greater than control values obtained for the intact linea alba $(82.25 \pm 7.60 \mathrm{~N}$ vs $79.55 \pm 11.46 \mathrm{~N})$

Conclusion: The use of a biomaterial in midline laparotomy closure significantly improves biomechanical resistance. Used in high-risk patients or even prophylactically, this method could drastically reduce the incidence of incisional hernia. (This study was supported by grant CICYT MAT 2004-02114)

O049

\section{IMPLEMENTATION OF ERAS IN ELECTIVE COLONIC SURGERY}

J.W. Duijff, O.G. Guicherit, P.G. van Dijk

Bronovo Hospital, The Hague, The Netherlands

ERAS (enhanced recovery after surgery) was introduced in our hospital in 2005 as a care improvement project. The protocol covers multiple evidence based care elements with the objective to improve recovery after colonic surgery. We conducted a retrospective study to evaluate adherence to and effect of ERAS in our hospital. A specially trained nurse informed patients preoperatively about ERAS. Clinical lessons were organized for nurses, house officers and surgeons to improve adhesion to the protocol. From July 2005 to present, 76 patients were included. Median age was 69 (38-95) years. 36 sigmoid resections, 27 right hemicolectomies , 5 transversectomies, 5 left hemicolectomies and 3 ileocecal resections were performed. We compared this group to a control-group operated before introduction of ERAS (January-July 2004 , $\mathrm{n}=23$ ).

Adhesion to protocol: $89 \%$ of patients consumed a carbohydrate-rich drink (Pre-op $\AA$ ) preoperatively; $94 \%$ had epidural analgesic; anti-emetic medication was given directly post-operative in 55\%; an intra-abdominal drain was placed in $24 \%$. In $94 \%$ the nasogastric tube (NG tube) was removed directly postoperative. 
Patient outcome: day 0,1 and 2: nausea $(27,38,35 \%)$; indication for NG tube $(6,13,19 \%)$; need for intravenous fluids $(100,86,51 \%)$; sitting in a chair $(24,58,31 \%)$; walking on day $2: 58 \%$; oral fluid intake: day 0 : $45 \%>300 \mathrm{ml}$, day $1: 71 \%>1 \mathrm{~L}$; normal diet on day $1: 46 \%$.

On average, first defecation followed after 2.2 days. On day 3: $87 \%$ had no pain, $26 \%$ needed Acetaminophen only.

Control-group vs ERAS: discharge from hospital (median): on day 10 (616) vs 7 (2-38), $p=.004$; discharge within one week: 4 vs $43 \%, p=.002$; discharge after $>14$ days: 17 vs $9 \%$, n.s. Complications, control-group vs ERAS: ileus 17 vs $9 \%$ (n.s.); anastomotic dehiscence 4 vs $7 \%$ (n.s.); atrial fibrillation 4 vs $5 \%$; deceased: 0 vs 1 patient. Readmission was necessary for 6 patients from the ERAS protocol (10\%).

Conclusion: ERAS significantly shortens length of hospital stay. Complication rate was not affected by ERAS. Adherence to the protocol is incomplete. This is caused by changes in nursing and house-officer staff and by adherence to traditional views on postoperative care. By organising repeated clinical lessons for all staff, adherence to ERAS is improving.

\section{O050}

TEP VERSUS LICHTENSTEIN. THE LEVEL-TRIAL: A PROSPECTIVE RANDOMISED MULTI- CENTRE CLINICAL TRIAL

H.R. Langeveld ${ }^{1}$, W.F. Weidema ${ }^{2}$, L.P.S. Stassen ${ }^{3}$, H.J. Bonjer ${ }^{4}$

E.W. Steyerberg ${ }^{5}$, J. Lange ${ }^{5}$, J. Jeekel ${ }^{5}$

1 Reinier de Graaf Groep Delft, Delft, The Netherlands

2 Ikazia Hospital, Rotterdam, The Netherlands

3 Reinier de Graaf Groep Delft, Delft, The Netherlands

4 Dalhousie University, Halifax, Canada

5 Erasmus Medical Center Rotterdam, Rotterdam, The Netherlands

The LEVEL-trial: Correction of inguinal hernia; Endoscopic VErsus Lichtenstein: a prospective randomised multi-centre clinical trial, was performed to evaluate the potential advantages of the 'Total Extraperitoneal Procedure' (T.E.P.) versus the Lichtenstein-procedure.

660 patients with primary or recurrent (uni- or bilateral) hernias were randomised within a period of 44 months in 6 centres in the Netherlands for elective inguinal hernia repair. Follow-up was 17 months. Primary results were postoperative pain, complications, recurrence, quality of life, period until return to work and return to activities of daily life. Patients had more pain after Lichtenstein-procedure until 6 weeks postoperatively. $(p=0,01)$. After 1 year, there was still more pain at the incision-site. $(p=0,02)$ More peroperative complications were seen with TEP. $(p=0,00)$ This was mainly because of peroperative bleeding. Impairments of sensibility were seen with Lichtenstein-procedure up until 1 year postoperatively. $(p=0,00)$ Other complications were not significant. There was no difference in the number of re-operations. TEP: $11(3,3 \%)$, Lichtenstein $8(2,5 \%)$. $(p=0,5)$ and recurrence. TEP $10(3,0 \%)$, Lichtenstein $7(2,2 \%) .(p=0,5)$

400 patients were included into a supplementary quality of life and cost efficacy analysis. Evaluation of quality of life (Euroqol) showed no significant difference between both groups. $(p=0,2)$ Patients had more hours of absence of work until 6 weeks postoperatively after Lichtenstein-procedure. Lichtenstein: 87 hours, TEP: 63 hours. $(p=0.00)$ ADL-evaluation showed an advantage for TEP. $(p=0,00)$.

Conclusion: TEP is associated with less postoperative pain, less impairments of sensibility, faster recovery of daily activities (ADL) and quicker return to work in comparison to the Lichtenstein-procedure. The number of peroperative complications with TEP is higher. This did not result into more recurrences or re-operations. TEP is to be recommended as an efficient method for inguinal hernia repair. In this trial the Lichtenstein-procedure was not performed under local anaesthesia. Therefore it is disputable whether TEP is to be recommended in old patients and patients with a high ASA-classification.

\section{O051}

LAPAROSCOPIC APPROACH FOR TECHNICALLY DIFFICULT GALL BLADDER STONE DISEASE: THE ROLE OF LAPAROSCOPIC SUBTOTAL CHOLECYSTECTOMY

M.A. Elsebae, H.E. Ezzat, M.S.H. Hedaya, M.A.N. Nasr Theodor Bilharz Medical Research Institute, Cairo, Egypt

A total of 531 consecutive patients were operated upon by laparoscopic cholecystectomy for symptomatic gallbladder stone disease in our institute over the last five years. Technical difficulty was encountered in
55 of them (10.36\%). Parameters of difficulty at laparoscopic cholecystectomy in our study involved obscured anatomy of the Calot's triangle absence of a dissection plane between the gallbladder and the liver bed or the occurrence of intraoperative complication. The operation was concluded by either standard laparoscopic technique, laparoscopic subtotal cholecystectomy or converted to open cholecystectomy. Technically Difficult laparoscopic cholecystectomy could be completed in 13 of the 55 patients $(23.63 \%)$. In twenty-four of the patients conversion to open cholecystectomy was required $(43.63 \%)$. Laparoscopic subtotal cholecystectomy was performed for 18 of patients $(32.72 \%)$ who constituted $3.39 \%$ of the laparoscopic cholecystectomies performed at the same time period. There was no operative mortality. Two only of the eighteen patients operated upon by laparoscopic subtotal cholecystectomy have had postoperative local complications in the form of bile leak $(11.1 \%)$. None of the patients who underwent laparoscopic subtotal cholecystectomy developed symptoms attributed to biliary disease or post-cholecystectomy syndrome at a mean follow-up period of 28 (range 6-40) months. Laparoscopic subtotal cholecystectomy LSC is a safe and definitive operation to treat diseases of the gallbladder, whenever there are major difficulties in dissection. It prevents bile duct injuries and allows removal of a difficult gallbladder without the need for conversion to open procedure. Key words: Subtotal cholecystectomy; Laparoscopic, Open.

\section{O052}

\section{META-ANALYSIS AND SYSTEMATIC REVIEW OF THE RELATION- SHIP BETWEEN HOSPITAL VOLUME AND OUTCOMES IN CAROTID ENDARTERECTOMY}

\section{P.J.E. Holt'1 , J.D. Poloniecki², I.M. Loftus², M.M. Thompson² 1st George's Vascular Institute, London, United Kingdom}

Objectives: This study investigated the relationship between annual hospital volume and the outcome following carotid endarterectomy (CEA). Materials and methods: PubMed, EMBASE and the Cochrane library were searched for all articles on the relationship between hospital volume and outcome following CEA. Articles were included if they presented data on post-operative death and/or stroke rate and annual hospital volume for CEA. The review conformed to the QUOROM statement. The data were meta-analysed and a pooled effect estimate of volume on the stroke and/or death rate from CEA quantified.

Results: Twenty-five articles, encompassing 936436 CEA, were analysed. Significant relationships between death rate, stroke rate and annual hospital volume were revealed. Overall, the pooled effect estimate was odds ratio 0.78 [95\% confidence interval $0.64-0.92$ ], in favour of surgery at higher volume units, with a critical volume threshold of 79 CEA per annum.

Conclusion: Significantly lower death and stroke rates were achieved at hospitals providing a higher annual volume of CEA. Adherence to volume criteria for this procedure would have a significant impact on the provision of carotid surgery. Different volume criteria would be needed for different healthcare systems. Case-mix adjustment must be made to interpret the relationship between volume and outcome.

\section{O053}

\section{ACQUISITION OF LAPAROSCOPIC SUTURING SKILLS IS NOT INFLUENCED BY OTHER LAPAROSCOPIC SKILLS}

\author{
B. Tang ${ }^{1}$, L. Christie ${ }^{1}$, F. Carter ${ }^{1}$, A. Cuschieri ${ }^{2}$ \\ 1 University of Dundee, Dundee, United Kingdom \\ 2 Scuola Superiore S'Anna di Studi University, Pisa, Italy
}

Background: laparoscopic suturing skill is essential for performing advanced laparoscopic procedures. Laparoscopic suturing and knot-tying skills can be acquired and improved by attending a well-structured short laparoscopic training course. However, in general belief, the trainees who have gained competence in basic laparoscopic skills are most proficient at acquiring laparoscopic suturing and knot-tying skills.

Aim: the aim of this study is to investigate if the skill acquisition in laparoscopic suturing and knot-tying skills is influenced by other laparoscopic skills.

Methods: Seven tasks were assessed on 57 operative trainees who attended advanced laparoscopic training courses without any experience in laparoscopic suturing. Scores were obtained by pre and post course assessment using checklist derived based on Objective Structured Clinical Examination (OSCE) and Observational Clinical Human Reli- 
ability Assessment (OCHRA). Evaluated task performances consisted of various cognitive (computer-based assessment) and operative skills including dissection, clipping, looping and laparoscopic suturing and knot-tying skills.

Results: Scores were obtained on each task by the pre and post course assessment using the checklists developed based on OSCE and OCHRA. There were no correlation observed between laparoscopic suturing skills and laparoscopic skills (cognitive knowledge, dissection, looping and clipping) in the pre and post course OSCE and OCHRA assessment. Strong correlation showed between the pre and post course assessment on cognitive knowledge in laparoscopic surgery (Pearson Correlation $r=0.612 ; \mathrm{P}<0.001$ ). Difference between the pre and post course assessment scores in all the 7 tasks assessed was significant $(\mathrm{P}<0.001)$

Conclusion: laparoscopic suturing skill is not influenced by other cognitive and operative laparoscopic skills. Laparoscopic suturing skill could be obtained and improved by attending a training course regardless the prior experience and skills in laparoscopic surgery.

\section{O054}

MEDIALLY APPROACHED RADICAL LYMPH NODES DISSECTION ALONG THE SURGICAL TRUNK FOR ADVANCED RIGHT-SIDED COLON CANCERS

J.K. Kawamura ${ }^{1}$, S. Nagayama², S. Hasegawa ${ }^{2}$, A. Nomura ${ }^{2}$, H. Inoue ${ }^{2}$,

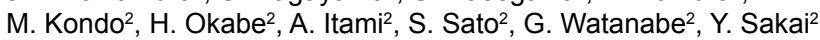

1 Kyoto University, Kyoto, Japan

Introduction: Although laparoscopic surgery for colorectal cancers is considered to be one of the treatment options, some technical problems remain to be solved as to radical dissection of regional lymph nodes(LNs), which is essential for improvement in treatment outcomes. We will present a secure procedure of laparoscopic right hemicolectomy to dissect regional LNs along the superior mesenteric vein (SMV).

Materials and methods: Twenty three patients underwent laparoscopic right hemicolectomy $(n=15)$ or extended right hemicolectomy $(n=8)$ for right-sided colon cancers in our institution between July 2005 and October 2006. Operative procedures: Five cannulae are required with the camera port placed in the infraumbilical position and the other 4 ports on both sides $(2 \times 2)$ in the lower abdomen. The surgeon uses the left sided cannulae and the assistant, the right sided cannulae. The most important thing to keep in mind is that the proper tension should be created and maintained by the cooperation of both hands of the assistant and the surgeon's one hand (tissue triangulation) during the procedure. The characteristic of our procedure is that all right and middle colic vessels are cut along the surgical trunk through the medial approach only. Firstly the 'ridge' of ileocolic vessels is identified and the mesocolon is dissected between the ridge and the periphery of SMV to expose the second portion of the duodenum. The ileocolic vessels are cut at their root. The ascending mesocolon is separated from the retroperitoneal tissues, duodenum and pancreas head up to the hepatocolic ligament cranially. The most important thing is the wide separation between the pancreas head and transverse mesocolon. This procedure unveils the course of right colic artery, veins and the gastrocolic trunk. The right colic artery and veins can be then cut at their root with safe. For extended right hemicolectomy, middle colic vessels are easily identified below the lower edge of pancreas and cut at their root. The ascending colon is mobilized by dissecting the lateral peritoneum and gastro- and hepatocolic ligaments, and then is taken off from the extended incision at the umbilicus, followed by en-bloc resection of the tumor and functional end-to-end anastomosis.

Results: We performed curative resection in this manner for consecutive 23 patients of advanced right-sided colon cancer without any intraoperative serious complications, and the postoperative course was uneventful in all patients. No case was converted to open surgery due to the technical difficulty or uncontrolled bleeding.

Conclusion: Laparoscopic radical LNs dissection for advanced rightsided colon cancers can be safely completed through the medial approach only. We think this is a safe method for radical LNs dissection in laparoscopic right hemicolectomy.

O055

A DECISION SUPPORT SYSTEM IN PRE-OPERATIVE ASSESSMENT OF ENDOVASCULAR AAA REPAIR

S.G.J Rödel ${ }^{1}$, E.E. Kunst ${ }^{2}$, R.H. Geelkerken ${ }^{3}$

1 UMC Groningen, Groningen, The Netherlands
2 Vrest B.V., Enschede, The Netherlands

3 Medical Spectrum Twente Enschede, Enschede, The Netherlands

In order to obtain an uncomplicated passage and a durable exclusion of an AAA through endovascular aneurysm repair (EVAR) one has to take into account many unique anatomical properties of the aortic-iliac-femoral trajectory and many unique properties of the stent-prosthesis itself. A stent Decision Support System can offer support in choosing the optimal type of stent-prosthesis, and in planning the procedure. We developed a computer based Decision Support System to guide vascular surgeons and trainees in their assessment of patients for endovascular treatment of abdominal aortic aneurysm (AAA).

We will present the first results and validation of this system for use in patient selection for EVAR.

Methods: In validating the system we used the anatomical CT and angiografic AAA criteria measured in 202 patients from two endovascular centres. Using a standard template each AAA was divided in ten segments. Five experts (surgeons and interventional radiologists) evaluated these cases and predicted the suitability of the EVAR intervention for each case. For each of the measured AAA parameters in each segment a quantified suitability rating profile was constructed using expert knowledge. These profiles were based on five years of 'expert clinician' experience of a Medtronic proctor centre (Medical Spectrum Twente) and were implemented in the Decision Support System. If available in the instruction manual the manufacturer information was incorporate in this segment profile. The system combines each segment parameter with the segment profiles for three different types of stent-prosthesis available. This result in a unique suitability rating prediction for placing a stent-prosthesis adapted for each individual stent-prosthesis segment configuration. We compared the suitability ratings of the decision support system with the assessment of five clinicians using the same anatomical data.

Results: There was an agreement in advice of $71.6 \%$ between the experts and the decision support system. In $0.2 \%$ the system advices false positive to operate in contrary of the experts opinion. In $24.6 \%$ the experts recognised the case as very difficult but possible to operate. The system was more safe and concluded not to advice operation. In $3.7 \%$ the system advices false negative not to operate in contrary of the experts. Between individual experts and the decision support system the kappa value ranged between 0.28 and 0.58 .

Conclusion: There appeared to be a good correspondence between the assessments of the Decision Support System and the clinical judgement. Specifically the chances for an incorrect positive advice from the expert system is minimal (1:500 assessments). In case to case suitability assessment between individual experts and the decision support system further refinements will be necessary. We believe a decision support system can reduce clinical inconsistency in individual EVAR decision strategy.

\section{O056}

FENESTRATED AND BRANCHED ENDOVASCULAR AORTIC ANEURYSM REPAIR: MID-TERM RESULTS

\section{C.J. Zeebregts, E.L.G. Verhoeven, I.F.J. Tielliu, W.T.G.J. Bos, B.E.} Muhs, R. Prins, J.J.A.M. van den Dungen

University Medical Center Groningen, Groningen, The Netherlands

Purpose: Fenestrated and branched endovascular aneurysm repair has emerged as an alternative treatment for aortic aneurysms with a short or absent neck. Although technically successful, longer-term results have been lacking. This article reports on the mid-term results of aneurysm repair with fenestrated and branched endografts from a European referral center with a large endovascular experience.

Materials and methods: From 2001 through 2006, a total of 45 patients, 36 male and 9 female, with a mean (SD) age of 73.5 (5.9) years were prospectively enrolled in a single institution database. Indications for fenestrated or branched EVAR included an abdominal aortic aneurysm $>5.5 \mathrm{~cm}$ with an unfavorable anatomy for traditional EVAR. Customized stent-grafts were either fenestrated or branched and based on the Zenith system. Data were analyzed on an intention-to-treat basis. Differences between groups were determined using paired student $T$ tests with $\mathrm{P}<.05$ considered significant.

Results: The mean (SD) follow-up was 26.7 (2.8) months and none of the patients were lost to follow-up. One patient died within 30 days due to mesenteric ischemia $(2.2 \%)$. All cause mortality was $22 \%$, mainly as a result of cardiac or metastatic disease. Out of the 114 targeted 
side-branches, one accessory renal artery appeared occluded at the end of the procedure. In the same patient, the main renal artery on the same side occluded within one month resulting in ipsilateral kidney loss. Post-operatively another 4 vessels occluded with one salvaged during reintervention, resulting in the loss of three additional kidneys. Mean serum creatinine level remained stable at 6 months, 1 year, and 2 years $(\mathrm{P}=\mathrm{NS})$. None of the patients required dialysis. There were 13 type 2 endoleaks. No other types of endoleak were detected. Mean aneurysmal sac size decreased significantly from 62.3 to $56.0 \mathrm{~mm}$ after 6 months and $47.1 \mathrm{~mm}$ after 2 years $(P<0.001)$.

Conclusion: The repair of AAA with fenestrated and branched endografts incorporating the visceral arteries is safe. Mid-term results are encouraging and support their continued use in patients with anatomic contraindications for standard EVAR. Long-term intensive surveillance is mandatory for early identification of visceral or branched vessel stenosis and pre-occlusion. Randomized clinical trials will eventually determine the efficacy and stability of this system.

\section{O057}

\section{ENDOLUMINAL FUNDOPLICATION FOR THE TREATMENT OF} GASTRO-OESOPHAGEAL REFLUX DISEASE

E.E. de Vries, B.P.L. Witteman, R.M. van Dam, W. Hameeteman,

G. Koek, N.D. Bouvy

Academic Hospital Maastricht, Maastricht, The Netherlands

Background: Recently the Esophyx has been introduced as a new technique to allow non-surgical, endoluminal fundoplication for the treatment of gastro-esophageal reflux disease (GERD). We report the first results of GERD patients treated with this device.

Method: Pre-operatively patients were selected having a small hernia diafragmatica $(<5 \mathrm{~cm})$, having an abnormal 24 hours $\mathrm{pH}$ metry and not willing to use lifelong medication.

Procedure: Under general anesthesia with the endoluminal device over the endoscope, in a retroflexed view, a full thickness fundoplication of at least 180 degrees circumference can be created. Immediately before and after ELF, valve grading was performed according to the Hill classification (grades I-IV).

One week after, patients were asked to stop their PPI's. Parameters to evaluate efficacy (before and till 3 months after ELF) were: 24 hours $\mathrm{pH}$ metry, GERD specific quality of life score (GERD-HRQL) and use of PPI. Results: At present 18 GERD patients, mean age 51 years (range 32-79) (7 female) were treated with ELF. ELF was performed safely in all 18 patients without serious complications. Intra-operatively solid full-thickness fundoplication over mean 200 degrees (range 180-220) was achieved in all patients. The Hill grade decreased from mean 2.5 before to mean 1 after ELF procedure. Mean procedure time was 91 minutes (range 55-145). During short term follow up (3 weeks) GERDHRQOL improved significantly from mean 31 to $16(p<0.01$; range $0-80)$. Thirteen patients $(73 \%)$ were able to stop all medication and in 5 patients PPI dose could be reduced by $50-75 \%$. When analyzing the results in more detail, it is apparent that patients with small hiatal hernia $(<3 \mathrm{~cm})$ fared better than those with larger hernia $(3-5 \mathrm{~cm})$. Eight out of 9 patients in the group with small hernia were able to stop all medication. Acid exposure time in the group with small hernia decreased sign from 8.0 to $4.7 \%(p<0.05)$.

Conclusion: The EsophyX ${ }^{\mathrm{TM}}$ ELF appears to be a safe and efficacious endoluminal procedure to control GERD on short time follow up. Best results are obtained in patients with small hiatal hernia $(<3 \mathrm{~cm})$.

\section{8}

ACCESS FLOW, VENOUS SATURATION AND DIGITAL PRESSURES IN HEMODIALYSIS

F. Van Hoek ${ }^{1}$, M.R.M. Scheltinga ${ }^{2}$, M. Luirink ${ }^{2}$, C.H. Beerenhout ${ }^{2}$

1 UMC St. Radboud Nijmegen, The Netherlands,

2 Maxima Medical Center, Veldhoven, The Netherlands

Introduction: A hemodialysis arteriovenous fistula (AVF) requires surgical modification in patients with cardiac overload $(\mathrm{CO})$ or dialysis access-associated steal syndrome (DASS). Creation of an artificial stenosis ('banding') within the AVF may be used, but this technique lacks the guidance of objective parameters. The aim of this pilot study was to identify parameters that reflect AVF flow in dialysis patients with either access related $\mathrm{CO}$ or DASS requiring corrective surgery.

Materials and methods: Patients underwent serial measurements of subclavian venous saturation (Satven), access flow (Flowus) and index digital pressures (Pdig) during a corrective banding procedure. Results: Data were obtained in 14 individuals ( 9 males, mean age: $53 \pm 6$ y) during 16 studies (CO $n=8$, DASS $n=8$ ). Prior to surgery, correlations between Flowpreop, Satven and Pdig were not significant. Stepwise banding of the AVF altered Satven in both groups from a mean of $91 \pm 1 \%$ (open AVF) to $84 \pm 2 \%$ (closed AVF, p<0.001). CO-patients demonstrated a lager drop (-13\%) compared to DASS-patients $(-4 \%)$. Values of Pdig increased from $68 \pm 9$ to $90 \pm 9 \mathrm{mmHg}(p<0.001)$, and both groups demonstrated a similar $+23 \mathrm{~mm} \mathrm{Hg}$ increase. In concert, the digital brachial index also significantly improved in al patients from $0.60 \pm 0.09 \%$ to $0.74 \pm 0.10 \%$. Linearity was present between alterations in Flowus and Satven in all patients but mostly in CO-patients ( $r 2=0.96$ ). Conclusion: Stepwise banding of hemodialysis fistulas leads to dose-dependent decreases in flow and ipsilateral subclavian venous saturation combined with augmented digital pressures in patients with cardiac overload and dialysis associated steal syndrome. Intraoperative measurements of venous saturation and digital pressures may have potential of guiding surgical correction in these patients groups.

\section{O059}

HISTOLOGICAL CHARACTERIZATION OF AORTIC ABDOMINAL ANEURYSM (AAA): INFLUENCE OF DIAMETER AND INTRALUMINAL THROMBUS

F.A.M.V.I Hellenthal, I.L.A. Geenen, G.W.H. Schurink, J.P. Cleutjens, S. Heeneman

University Hospital Maastricht, Maastricht, The Netherlands

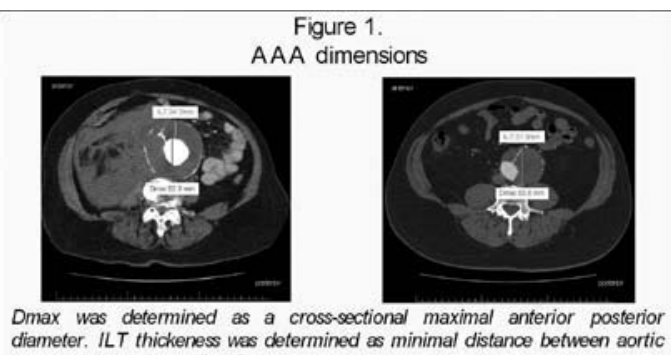

Diamer. ILT thicheness is a chowisectional maximal anterior postenior lumen and anterior wall at Dmax.

Figure 1

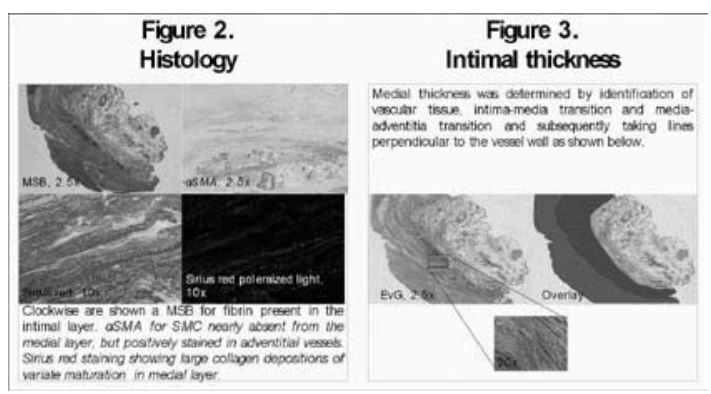

Figure 2 and 3

AAA is characterized by disruption of medial elastin, gross medial collagen deposition and inflammatory cell infiltration. Furthermore, neovascularization is a striking feature for which hypoxia of the vessel wall as underlying mechanism has been implicated. Normal aortic intima and inner media are oxygenized by diffusion from the aortic lumen and in this respect intraluminal thrombus (ILT) is thought to cause hypoxia of these layers. We investigated how histological characteristics correlate to clinical characteristics of AAA including size (Dmax) and (ILT) thickness. Furthermore, we directly tried to demonstrate any hypoxia of the aortic wall by means of hypoxia inducible factor $1- \pm$ (HIF-1 \pm ).

Thirty-seven consecutive patients undergoing conventional aortic reconstructive surgery were included. Patients undergoing redo procedures or who had proven inflammatory aneurysms were excluded $(n=2)$. Patient and AAA characteristics were collected from medical records and CTA images (Figure 1). Tissue was collected from the anterior aneurysm wall at Dmax, conserved in formaldehyde and embedded in paraffin. Sections were stained for nuclei and cytoplasma (Hemotoxiline and Eosine, $\mathrm{HE}$ ), elastine (Elastine van Gieson, EvG), fibrine (Martius/Scarlet/Blue, $\mathrm{MSB}$ ), collagen (Sirius red), smooth muscle cells (SMC) ( \pm -smooth muscle cell actin, \pm -SMA) and hypoxia (HIF-1 \pm ). Intimal and medial thickness were determined by two observers by means of an in house 
developed programme for Quantimet. Linear regression analysis was performed (Figure 3 ). Mean age was 70 years (55-80) and male to female ratio was 32:3. Mean Dmax and mean ILT thickness were $70.4 \mathrm{~mm}$ (50.0-94.3) and $25.6 \mathrm{~mm}$ (2.2-50.0), respectively. EvG and \pm SMA staining showed total destruction of elastic laminae and absence of SMCs in the medial layer. Sirius red staining confirmed that this layer consisted of collagen of several grades of maturity (Figure 2). The intimal layer was thickened by fibrin, no SMCs were identified here. All sections showed positive staining for HIF-1 1 . Mean medial thickness was 198.3 (52.72'1306.35). Using linear regression analysis no significant relation was found between medial thickness, Dmax and ILT thickness The degree of elastine destruction, collagen deposition, absence of SMC and positive staining for HIF-1 \pm was equally distributed in all AAA.

Our results suggest that on a histological level infrarenal AAA can not be classified in analogy to clinical characteristics. Medial thickness does not seem to have any relation to Dmax and ILT thickness. Independent of Dmax and ILT thickness all sections stained positively for HIF-1士. However, all included AAAs had ILT. It is possible that even a minimal amount of ILT disrupts diffusion of oxygen from the aortic lumen to the aortic wall. In future studies the oxygen demand of the aortic wall can be estimated and the diffusion capacity and maximal diffusion distance of ILT can be calculated by means of the first law of Fick. Additionally, in analysing the stained sections we encountered the problem defining the intima and media, due to absent landmarks of the elastic laminae. Last, it is to be kept in mind that we are looking at end stage disease.

\section{O060}

THE ABDOMINAL AORTIC ANEURYSM: A CONSPIRACY OF PROTEASES

H. Abdul-Hussien ${ }^{1}$, J.H.N. Lindeman², R. Hanemaaijer ${ }^{3}$, J.H. Bockel ${ }^{2}$ The Hague, The Netherlands

2 LUMC, Leiden, The Netherlands

3 TNO, Leiden, The Netherlands

Growth and rupture of abdominal aortic aneurysm (AAA) result from progressive loss of structural collagens that is not adequately matched by collagen deposition. Collagens are highly stable molecules that require cleavage by specific collagenases. Yet the collagenases responsible for the increased collagen turnover in AAA have not been identified.

Methods and results: we systematically analyzed collagenase expression and activity in AAA and ruptured AAA by mRNA analysis, and used specific protein-activity assays and Western blot analysis to address the posttranslational regulation of proteases activity. This study identifies neutrophil collagenase (MMP-8) and the cysteine collagenases Cathep$\sin \mathrm{K}, \mathrm{L}$ and $\mathrm{S}$ as the principal collagenolytic culprits in AAA and ruptured AAA. Extracellular activities of the cysteine collagenases critically depend on an acidic microenvironment. In osteoclasts such an environment is created by the transmembrane-ous proton pump v-H+ATPase. Co-expression of the osteoclast proton pump with cysteine collagenases in mononuclear cells and smooth muscle cells shows that conditions required for extracellular cysteine protease activity exist in AAA. Deficiencies in cystatin $C$, the principle inhibitor of cysteine protease activity, may further contribute to the proteolytic imbalance in AAA. We show that the origin of this deficiency is not at transcriptional level, but relates to cystatin $\mathrm{C}$ degradation by neutrophil collagenase and elastase. Conclusion: MMP-8 and the cysteine collagenases Cathepsin K, L and $S$ are the principal collagenases in AAA and ruptured AAA. Observed cystatin $C$ deficiencies in AAA are secondary and relate to cystatin $C$ degradation.Identifying mechanisms that lead to aneurysmal progression in humans is crucial to identify targets for effective medical therapy to inhibit aneurysmal growth and prevent surgery in a large group of patients. Supported by a grant from The Netherlands Heart Foundation (NHS 2000B165)

\section{O061}

\section{ENDOTHELIAL PROGENITOR CELL RESPONSE TO VENOUS THROMBOSIS}

A. Wadoodi, D. Patel, M. Waltham, K. Burnand, A. Smith St Thomas' Hospital, London, United Kingdom

Background: Thrombus resolution occurs through a process of neovascularisation. Endothelial progenitor cells (EPCs) are thought to play a role in this process. EPCs are identified by the co expression of three markers: SCA-1 (bone marrow), CD34 (stem cell) and VEGFR2 (endothelial cell). This study examines the changes in circulating EPCs following venous thrombosis.

Methods: Thrombus was induced in two groups of six mice using a combination of reduced flow and vessel wall damage. Two groups of six control mice underwent a sham control operation. Blood and bone marrow were collected at 24 and $48 \mathrm{hr}$ post-operatively and processed for flow cytometry, using anti-SCA-1, anti-CD34 and anti-VEGFR2. Cells were counted on a FACS Calibur (Beckmann) and analysed using the Win'MDI software package. EPC's were counted in the mononuclear region of the scatter plot, and presented as a percentage of the total number of cells in that region. The numbers of circulating progenitors were compared using a Mann Whitney U-test.

Results: There is a 25 fold increase in the number of (SCA-1+/CD34+/ VEGFR2+) EPCs in thrombus induced animals compared with sham controls at day 1 after operation. The percentage of EPCs in thrombus induced animals was $0.378 \%$ (range $0.12-0.76$ ) compared with $0.015 \%$ (range $0.01-0.13$ ), $\mathrm{P}=0.004$. The bone marrow at day one post operation did not demonstrate a significant difference between the sham control group and the thrombus group.

Conclusion: Venous thrombosis in this animal model results in a significant increase in the number of circulating endothelial progenitor cells. The bone marrow does not reflect this early rise in EPCs using conventional markers; this may suggest that the EPC phenotype only develops once the bone marrow cells enter the circulation. A better understanding of how progenitor cells are involved in thrombus resolution may allow us to enhance resolution by stimulating progenitor release.

\section{O062}

EVOLUTION OF AORTIC WALL MORPHOLOGICAL CHARACTERISTICS RESULTING FROM PROLONGED PROPRANOLOL

\section{TREATMENT}

K.M. Lampropoulos, D.P. Sokolis, V. Papalouka, C.A. Dimitriou,

M. Kyriakides, P.E. Karayannacos

Center of Experimental Surgery, Foundation of Biomedical Research, Academy of Athens, Athens, Greece

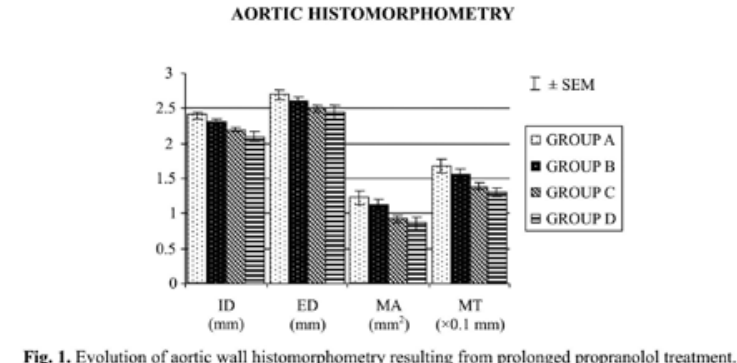

AORTIC COMPOSITION

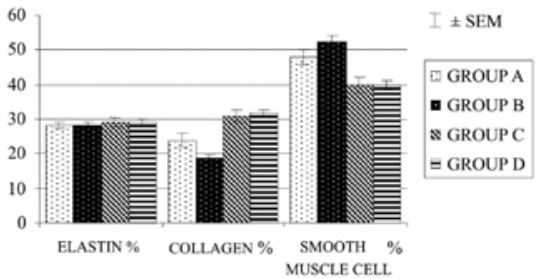

Fig. 2. Evolution of aortic wall composition resulting from prolonged propranolol treatment.

Aim: Previous studies have focused their interest on the influence of sympathetic nerves, but not of ${ }^{2}$-receptors, on the structure and function of the aorta. The aim of the present study was to examine the time course of morphological changes occurring in the aortic wall in response to chronic pharmaceutical administration of propranolol.

Materials and methods: Sixty-six healthy male Wistar rats of the same age and weight were separated into 4 groups. Group A (control) was divided in 3 subgroups $A 1(n=6), A 2(n=6)$, and $A 3(n=6)$, with animals receiving only water with their diet. In groups $B(n=16), C(n=16)$, and $D(n=16)$, propranolol was added in a dose of $100 \mathrm{mg} / \mathrm{kg} /$ day in their drinking water. Sufficiency of ${ }^{2}$-blockade was assessed by examination of the effect of isoproterenol (iv, $0.5 \mu \mathrm{g}$ ) on heart rate. Animals of groups $A 1$ and $B, A 2$ and $C$, and $A 3$ and $D$ were euthanized after 1,2 , and 3 months respectively. The thoracic aorta was resected and rings were obtained at the level of the 3rd pair of intercostals that were fixed in for- 
maldehyde, embedded in paraffin, and stained with Verhoeff's elastica, Sirius red, and hematoxylin-eosin. Transverse sections were examined on a light microscope, and the internal (ID) and external diameters (ED) of the vessel, as well as the thickness of tunica media (MT) and its cross-sectional area (MA) were measured with an image processing system. Quantification of aortic wall composition included measurement of percentage collagen, elastin, and smooth muscle cell content, number of lamellar units and of smooth muscle cell nuclei.

Results: $\beta$-blockade was sufficient in group $B, C$, and $D$ animals. Morphological parameters were invariant in subgroups $A 1, A 2$, and $A 3$; thus were pooled as control group. ID and ED of the vessel progressively decreased from the control group to group D (ANOVA: $p<0.05$; Fig. 1). Similarly, MT and MA progressively decreased from the control group to group D (ANOVA: $p<0.05)$.

Smooth muscle cell content of the aortic wall gradually decreased from the control group to group D, while collagen content increased (ANOVA $p<0.05$; Fig. 2). No change was noted in elastin content, and in the number of lamellar units and of smooth muscle cell nuclei.

Conclusion: Chronic blockade of ${ }^{2}$-adrenergic receptors induced morphological and compositional changes in the thoracic aortic wall from the 1st month of propranolol administration. Maximum changes were observed in the 3rd month and expressed by a reduction in vessel dimensions and smooth muscle cell content, and by an increase in collagen content. It is concluded that the sympathetic system and its ${ }^{2}$ -receptors have a decisive role in the morphology and composition of the vessel and accordingly in its function, and should, therefore, be considered in clinical practice.

\section{O063}

\section{PRESERVATION OF MOLECULAR STRUCTURE OF ARTERIAL} GRAFTS IN PULVERIZED SODIUM CHLORIDE

M. Gewartowska, M. Maksymowicz, W.L. Olszewski

Medical Research Center, Warsaw, Poland 2 Central Clinical Hospital, Warsaw, Poland

There is a desparate clinical requirement for biological arterial grafts. Artificial grafts have a limited time patency due to a continuing foreign body process at the site of implantation. In our previous studies skin, spleen and liver fragments dehydrated in anhydric $\mathrm{NaCl}$, kept at room temperature for months and rehydrated in $0.9 \% \mathrm{NaCl}$ supplemented with BSA retained their molecular structure. Moreover, dehydrated skin fragments preserved for 10 months and transplanted to SCID mice were taken by recipient.

Aim: To establish a method of successful preservation of aorta for long time periods with unchanged morphological structures and reactivity. Methods: Freshly obtained rat fragments of rat aorta were salinated in anhydric sodium chloride powder and stored at $4 \mathrm{oC}$ for 48 hours up to 2 months. Samples were desalinated in $0.9 \% \mathrm{NaCl}$ supplemented with $5 \%$ BSA. Morphology was evaluated on cryosections using hematoxyline / eosine staining method. Cryosections were stained using mAbs anti CD 31, factor VIII and actin as well as trichrome staining for collagen and Gomori staining for elastic fibers. Syngeneic transplantations of rat aorta were performed, samples were collected 7 days to 3 months after transplantation.

Results: Hematoxyline / eosine staining showed preserved anatomical structure of aortas after storage in dehydrated $\mathrm{NaCl}$ for seven days up to two months. There were no significant differences between fixed and non-fixed aortas in antibody staining against CD 31, factor VIII and actin as well as trichrome. The orthotopically transplanted aortas pulsated after 7 days to 3 months after transplantation. Histology revealed weak staining for actin, the collagen and elastic fibers retained their architecture. There was no positive staining for endothelial cell HIS52. The tensile strength of harvested specimens did not differ from controls. Conclusion: These data showing perfectly preserved anatomical and molecular structure of aortas stored at $40 \mathrm{C}$ for months and successfully transplanted, indicate that dehydration in anhydric $\mathrm{NaCl}$ may be considered as a novel method for preservation of arterial grafts.

\section{O064}

BACTERIAL COLONIZATION OF TISSUES OF CHRONICALLY ISCHEMIC LOWER LIMB

W.L. Olszewski ${ }^{1}$, P. Andziak ${ }^{2}$, M. Durlik ${ }^{1}$, M. Garlicki ${ }^{3}$, B. Interewicz ${ }^{1}$,

E. Swoboda ${ }^{3}$, E. Stelmach ${ }^{3}$

1 Medical Research Center, Warsaw, Poland

2 Central Clinical Hospital, Warsaw, Poland

\section{Medical University of Warsaw, Warsaw, Poland}

Reaction of arterial wall to bacterial infection as etiological factor in pathogenesis of atherosclerosis remains a contentious issue. The majority of available data on identification of bacterial antigen originates from studies of coronary arteries. Only few studies have been devoted to investigation of lower limb arteries, despite of a high incidence rate of atherosclerosis in these vessels.

Aim: to investigate the presence of bacterial cells and microbial DNA with the use of broad-range PCR, targeting conserved region (16sRNA) of the gene encoding for ribosomal RNA and specific primers to detect Chlamydia pneumoniae (CP) and Helicobacter pylori (HP) in fragments of femoral and popliteal arteries of patients undergoing reconstructive surgery or amputation. As control served carotid artery and aortic plaques.

Methods: Using routine microbiological methods popliteal and femoral arteries contained isolates (Staph. epidermidis, aureus, Enterococcus, Pseudomonas), carotid arteries $4.1 \%$ and aorta $0.7 \%$. One hundred patients were studied. Fragments of arteries were harvested under strictly sterile conditions. A part of each fragment was cultured, from the remaining DNA was extracted. PCR amplification was performed with primers for gene fragment coding bacterial 16s RNA, major outer membrane protein (ompA) of CP and urease gene of HP DNA with positive and negative controls. Products were separated by PAGE electrophoresis and silver stained. Routine bacteriological cultures of specimens were also carried out. All patients undergoing lower limb arteries were treated with antibiotics

Results: Microbial DNA (16sRNA) was detected in $64 \%$ of examined femoral and popliteal specimens. CP could be demonstrated in $69 \%$ of positively-tested patients while HP was detected in $3.8 \%$. In carotid arteries $29 \%$ of cases contained bacterial DNA, 29\% CP and 0\% HP specific genes. Thirty one aortic specimens contained bacterial DNA, $65 \% \mathrm{CP}$ and $18 \% \mathrm{HP}$

Conlusion: Bacterial isolates and DNA were found in lower limb arteries. Aorta and carotid arteries only sporadically contained isolates. The microbes colonizing limb vascular bundles may be responsible for the postoperative infective complications after arterial surgery.

\section{O065}

A STUDY OF FAECAL INCONTINENCE IN PATIENTS WITH CHRONIC ANAL FISSURE: ITS RELATION TO THE EXTENT OF INTERNAL ANAL SPHINCTER DIVISION DURING LATERAL SPHINCTEROTOMY

\section{M.A. Elsebae, H.H. Helmy}

Theodor Bilharz Medical Research Institute, Caïro, Egypt

Background: Troublesome faecal incontinence following a lateral internal sphincterotomy is often attributed to faulty surgical technique. However, it may be associated with coexisting occult sphincter defects Whether, continence is related to the extent of sphincterotomy remains debatable. Objective: Our aim is to identify faecal incontinence related to chronic anal fissure prior and after lateral internal sphincterotomy and its relation to the extent of internal anal sphincter division.

Methods: 108 patients with chronic anal fissure were prospectively studied before and after lateral internal sphincterotomy up to the dentate line in 52 and up to the fissure apex in 56 of patients. A questionnaire was completed for each patient before and after surgery with regard to any degree of faecal incontinence such as soiling of underclothes, control of flatus and accidental bowel motion.

Results: Minor degrees of incontinence were present prior to surgery in 16 of patients $(14.8 \%)$ and in $22(20.3 \%)$ patients after surgery; the majority of them were incontinent before surgery. Sphincterotomy up to the dentate line caused a significant change in anal incontinence $(8.9 \%)$ compared to only $(2.77 \%)$ of the partial sphincterotomy patients $(P=0.016)$. Temporary postoperative incontinence was reported in six of patients. All of them were females. Persistent incontinence to gas occurred in two of them $(3.57 \%)$. All have had a history of one or more vaginal delivery. Conclusion: A mild degree of incontinence is a feature of chronic anal fissure rather than a result internal sphincterotomy. Sphincterotomy up to the dentate line provided a faster healing, but it was associated with a significant alteration in anal continence than sphincterotomy up to the fissure apex. Care should be exercised especially in female patients with a history of previous obstetric trauma, as internal anal sphincter division may further compromise sphincter function. 
Keywords: Chronic anal fissure, lateral internal sphincterotomy, Incontinence.

\section{O066}

THE ADHESION POWER OF DIFFERENT PROSTHETICS MATERIALS FOR HERNIA SURGERY IMPLANTED IN PERITONEAL PERITONEUM

L. Ansaloni ${ }^{1}$, F. Gazzotti ${ }^{1}$, F. Catena1, F. Coccolini ${ }^{1}$, S. Di Saverio ${ }^{1}$, M. Fini ${ }^{2}$, S. Gagliardi ${ }^{1}$, R. Giardino ${ }^{2}$, A.D. Pinna ${ }^{1}$

1 St.Orsola-Malpighi University Hospital, Bologna, Italy

2 Rizzoli Orthopaedic Institute, Bologna, Italy

Table 1.

\begin{tabular}{llll}
\hline & adhesion percentage & adhesion grade & AI \\
PP & $81,3(33,5)$ & $3,5(1,2)$ & $318,8(144,3)$ \\
PP+ePTFE & $35,4(37,6)$ & $2,1(1,6)$ & $114,6(148,0)$ \\
PP+Surgisis & $16,7(31,2)$ & $0,5(0,8)$ & $20,8(35,1)$ \\
PP+Polyuretan & $3,1(7,8)$ & $0,3(0,6)$ & $5,2(14,6)$ \\
Control & $3,1(7,8)$ & $0,2(0,4)$ & $3,1(7,8)$
\end{tabular}

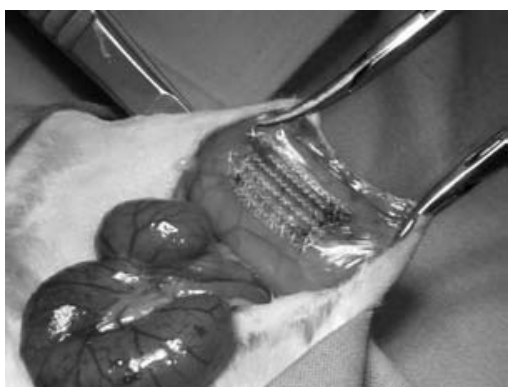

Background: Although for abdominal wall defects repairs polypropilene (PP) is the prosthetic material mainly used, its potential role in adhesion formation, when put on straight contact with bowel, is well known. For this reason other different prosthetic materials have been proposed where PP is coated by other meshes which can prevent adhesion formation. Aim of our work is to test the adhesions' formation to different prosthetic materials when implanted intraperitoneally.

Materials and methods: In our study we compare the adhesion formation due to PP with three other kind of composite prosthesis (PP+ePTFE, PP+Surgisis, PP+Polyuretan) and a group of control. Sixty $\mathrm{SD}$ male rats (12 for 5 groups) underwent laparotomy and denudation of the serosa of the cecum and peritoneal covering of the abdominal wall: three treated mesh products (PP+ePTFE, PP+Surgisis, PP+Polyuretan) and one untreated mesh (untreated $P P$ ) were randomly placed between the cecum and abdominal wall. A group without mesh was used as control [1]. The animals were sacrificed at 21 days, 3 and 6 months (4 at each time) following surgery and analyzed for the presence of adhesions. The adhesions were scored macroscopically in terms of extent (quantity) and type (quality) by two independent observers. The extent of adhesion formation was quantified by dividing the area to be scored into eight. When adhesions were present in one of the eight areas, a score of $12.5 \%$ was recorded [2]. The type of adhesion formed was classified as described by Zuhlke et al. [3]. The Adhesion Index (Al) is an overall measure of adhesion formation and is defined as the product of extent and quality of adhesions. Statistical analysis was performed by the Mann-Whitney U test for independent samples. Comparisons were made between treatment and control groups. Statistical significance was defined as $p<0.05$. Data were expressed as mean (SD) of adhesion percentage, adhesion grade and Al.

Results: The means (SD) of adhesion percentage, adhesion grade and adhesion index for each experimental group is reported in Table1. Regarding the Al, there wasn't a significant difference between either $\mathrm{PP}+\mathrm{Polyuretan}$, either PP+Surgisis and Control; instead the difference was significant for PP+ePTFE $(p=0,002)$ and PP $(p<0.001)$; moreover there wasn't a significant difference between PP+Polyuretan and $\mathrm{PP}+$ Surgisis, but both of them were significant diffent vs. PP+ePTFE (respectively $p=0,003$ and $p=0,033$ ); lastly $P P+e P T F E$ was significant different from PP $(p=0,010)$
Conclusion: Although PP+ePTFE, PP+Polyuretan and PP+Surgisis meshes appear to provide a better alternative to the use of PP meshes for intraperitoneal implant in terms of the formation of adhesions, PP+Polyuretan and PP+Surgisis meshes seem significant less adhesiogenic than PP+ePTFE.

References: 1. Gonzales R et al. Resistance to adhesion formation: a comparative study of treated and untreated mesh products placed in the abdominal cavity. Hernia 8:213-9, 2004

2. Vrijland WW et al. Peritoneal adhesions to prosthetic materials. Surg Endosc 14:960-3, 2000

3. Zuhlke HV et al. Pathophysiology and classification of adhesions Langenbecks Arch Chir Suppl II Verh Dtsch Ges Chir. 345:1009-16, 1990

\section{O067}

LONG TERM RESULTS OF STAPLED HEMORRHOIDOPEXY FOR THIRD AND FOURTH DEGREE HEMORRHOIDS

\section{J.W. Duijff, H.J. Smeets \\ Bronovo Hospital, The Hague, The Netherlands}

Circular stapled hemorrhoidopexy (SH) has shown good short-term results and quicker recovery compared to classic open hemorrhoidectomy. Long-term results ( $>2$ years) vary and have been scarcely reported. This study evaluates long term safety and efficacy of $\mathrm{SH}$ for prolapsing hemorrhoids. All 38 patients who underwent SH in our clinic from 20012005 received a questionnaire. We assessed symptoms in the preoperative period, in the first week after $\mathrm{SH}$, and at present. Patients were invited for long-term follow-up, consisting of interview, examination and proctoscopy. Satisfaction with the result of SH was indicated with a VAS score. 31 patients $(82 \%)$ returned the questionnaire; 24 patients $(63 \%)$ complied with a follow-up visit. Median follow-up: 45 (8-56) months. Population: $66 \%$ women; median age 55 (31-91) years. Patients rated their pain ( $1=$ no pain, $5=$ severe pain) and reported symptoms occurring at least once a week. Preoperatively: mean pain score $2.6( \pm 1.4)$, prolapse $97 \%$, bleeding $55 \%$, soiling $16 \%$, incontinence $16 \%$, constipation $29 \%$, pruritus $48 \%$. Complications: urinary retention $32 \%$, vasovagal collapse $5 \%$. First week after SH: mean pain score $2.7( \pm 1.5)$, prolapse $3 \%$, bleeding $36 \%$, soiling $16 \%$, incontinence $16 \%$, constipation $36 \%$, pruritus $13 \%$. Long-term follow-up: mean pain score $1.3( \pm 0.6)$, prolapse $13 \%$, bleeding $3 \%$, soiling $7 \%$, incontinence $7 \%$, constipation $10 \%$, pruritus $10 \%$. One of the two patients with postoperative soiling already had soiling preoperatively, as was the case for one of the two patients with postoperative incontinence. At long-term follow-up, no stenoses, fistulas or fissures were seen. Recurrent prolapse occurred in 5 patients (16\%). Three recurrences happened within a month after $\mathrm{SH}$, two after 44 and 50 months. Four out of five recurrences happened among the first 14 SH's performed in our clinic. Median VAS score was 85 (18-100). Conclusion: $\mathrm{SH}$ significantly reduces bleeding, pain, pruritus and prolapse $(p<.001)$. With a median follow-up of 45 months, this study shows that the effect is durable. A total of $16 \%$ recurrences is comparable to the literature which describes recurrence rates of 0.3 to $>50 \%$ with follow-up scarcely more than 2 years. Recurrent prolapse in this study can be explained by the casemix (only third and fourth degree hemorrhoids were treated) and learning curve effect. Except for the high risk of urinary retention, $\mathrm{SH}$ is safe.

\section{O068 \\ EFFECTS OF NUCLEAR FACTOR ?B INHIBITORS ON COLON ANASTOMOTIC HEALING IN RATS}

\section{A. Bedirli, B. Salman, M. Kerem, H. Pasaoglu, E. Ofluoglu, O. Sakrak Gazi University, Ankara, Turkey}

Background: The transcription factor nuclear factor-kB (NF-kB) plays an essential role in inflammation. To date, no studies have investigated the effect of inhibiting NF-kB-mediated inflammation on colonic anastomotic healing. We tested this by administering of NF-KB into rats undergoing a well-established model of colonic anastomotic healing. Methods: Ninety adult Wistar rats underwent laparotomy, descending colonic transection and handsewn reanastomosis. The animals were randomized to receive either a selective NF-kB inhibitors (parthenolide, $0.5 \mathrm{mg} / \mathrm{kg}$; resveratrol $0.5 \mathrm{mg} / \mathrm{kg}$ ) or an equal volume of water by gavage before operation and then daily after surgery. Animals were killed three, five or seven days after the operation. Wound healing was assessed by mechanical (bursting pressure), biochemical (hydroxyproline content, gelatinase activity), and histologic parameters. 
Results: No differences between groups were recorded for any of the parameters of wound healing on day three. On day seven, hydroxyproline levels were significantly higher in the parthenolide and resveratrol groups. A similar pattern was observed with the bursting pressure. In contrast, gelatinase activity (MMP-2 and MMP-9 expression) was significantly higher in the control group on postoperative day 5 . Histology revealed a significant increase of inflammatory cell infiltration, neoangiogenesis and fibroblast activity in the parthenolide and resveratrol groups compared with the control group. On day three, expression of NF-KB activity was upregulated in the anastomotic area. Both parthenolide and resveratrol completely attenuated NF-kB activity.

Conclusion: Parthenolide and resveratrol significantly improve healing and mechanical stability of colon anastomosis in rats during the early postoperative period. Both agents may be acting to accelerate host reparative process as well as to enhance protection of the anastomotic wound bed.

\section{O069}

\section{CT SCAN IN PATIENTS WITH SUSPECTED APPENDICITIS: CLINI-} CAL IMPLICATIONS FOR THE ACUTE CARE SURGEON

R.J. Mason, M. Petrosyan, J.J. Estrada, W. Yacoub, S. Somers, S. Chen University Of Southern California, Los Angeles, Usa

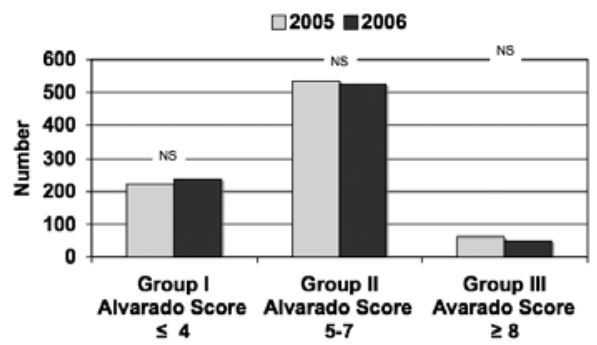

Frequency distribution by Alvarado Score

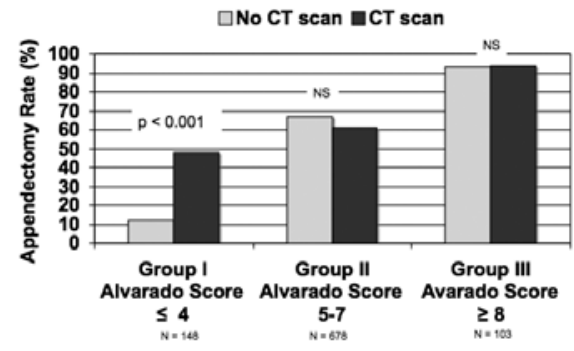

CT scan influence on appendectomy rate

Background: In the USA the diagnosis of appendicitis is increasingly reliant on computed tomography (CT), often obtained by Emergency Room physicians prior to consulting a surgeon. In addition, uncertainty of the diagnosis of appendicitis also necessitates the need to perform a CT scan. Standardized clinical tools, such as the Alvarado Scoring system, have been used to aid in the clinical diagnosis of appendicitis. Hypothesis: The recent increase in utilization of CT scans in patients with right iliac fossa (RIF) pain is associated with a decreased negative appendectomy rate.

Objective: To determine the influence of CT Scan on the management and outcome of patients suspected to have appendicitis.

Patients and methods: 1630 patients with suspected appendicitis were evaluated by the Emergency Surgical Service from December 2004 to December 2006. The Alvarado score was calculated for each patient at the time of presentation and patients were categorized into 3 groups: I - Appendicitis unlikely (Alvarado score $\leq 4$ ); II - appendicitis possible (Alvarado score 5-7) and III - appendicitis highly likely (Alvarado score $\geq$ 8). Computed tomography utilization, hospital course and final pathology results were reviewed.

Results: The number of patients who presented with right iliac fossa pain and the frequency distribution of the patients according to Alvarado score in 2005 were similar to that of 2006 (Figure 1). Significantly more patients received a CT scan in 2006 compared to 2005 (60 \% vs. $52 \%$; $p=0.001)$. The statistical difference was significant for patients in group II
(64 \% vs. $46 \%, p<0.001$ ) but not in groups I and III.

929 of $1630(57 \%)$ patients underwent appendectomy. The overall appendectomy rate was similar between the 2 years (57\% in 2005 vs. $57 \%$ in $2006 p=0.995$ ). The overall appendectomy rate in patients that received a CT was significantly higher when compared to those patients that did not receive a CT scan (60\% vs. $53 \%$, $p=0.002)$. The appendectomy rate in patients with Alvarado score of $\leq 4$ (group I) and no CT scan was significantly lower than the rate in patients with a same Alvarado score and a CT scan $(12 \%$ vs. $48 \%, p<0.0001)$. CT scan utilization made no difference on the appendectomy rate in patients from groups II and III (Figure 2). The overall negative appendectomy rate in patients who did not undergo a CT scan was similar to that of patients with a positive preoperative CT scan: 23 of $383(6 \%)$ vs. 31 of $546(6 \%)$ respectively.

Conclusion: CT scan utilization increased the appendectomy rate only in patients with low clinical suspicion for appendicitis, and had no influence on the frequency of appendectomy in patients with probable or highly likely clinical evidence of appendicitis. Furthermore, preoperative CT scan did not decrease the negative appendectomy rate in any patient with suspected appendicitis. Surgeons should still rely on clinical acumen in the management of patients with suspected appendicitis, and only use CT scan as an aid when the diagnosis is in question.

\section{O070}

\section{RESULTS OF MONOLATERAL DUAL KIDNEY TRANSPLANTATION TECNIQUE}

B.Ekser ${ }^{1}$, L. Fabris ${ }^{1}$, N. Baldan¹, L. Furian ${ }^{1}$, M. Vecchiato1, M.L. Valente ${ }^{2}$, P. Rigotti ${ }^{1}$

1 Kidney and Pancreas Transplantation Unit, Padova, Italy 2 Institute of Pathological Anatomy, Padova, Italy

Introduction: One option for using organs from elderly donors with a suboptimal nephron mass is dual kidney transplantation(DKT). DKT from elderly donors is increasingly used by many centers to cope with the unsolved organ shortage problem with excellent results in terms of renal function and patient survival rate, but the longer operating time and the greater surgical risk (due to two iliac accesses) of this technique are cause for concern. Placing both kidneys monolaterally has the advantage of a single surgical access, thereby reducing the surgical trauma and operating time, and surgical risk for older recipients. In this study, we retrospectively reviewed our patients who underwent monolateral placement of both kidneys (MPDKT).

Methods: 53 MPDKT were performed at our Center from June 2003 to November 2006, from donors with a mean age of $73 \pm 5$ years (range 5481). Our selection criteria were based on clinical evaluation of the donor and both macroscopic and histological assessment of the kidneys. Both kidneys were placed extraperitoneally in the same iliac fossa. The right kidney was transplanted with its extended renal vein placed superiorlaterally, while the left kidney was placed inferior-medially. Extravescical ureteroneocystostomies were then performed separately according to the Lich-Gregoir technique. The recipient mean age was $61.2 \pm 5.1$ years (range 48-72), and the mean operating time was $258 \pm 30$ minutes. Mean cold ischemia times for the 1 st and 2 nd kidney were $15.4 \pm 2.7$ and 16.2 \pm 2.6 hours, respectively. Mean HLA-mismatches were 4.6 \pm 1.1 . Calcineurin inhibitor-free, sirolimus based, immunosuppression was preferred in the majority of cases $(90.5 \%)$ to avoid nephrotoxicity, using ATG for induction therapy (94.3\%).

Results: The actuarial 2-year patient and graft survival rates, after a mean follow-up of $18 \pm 11$ months, are $97.7 \%$ and $91.2 \%$, respectively. We observed 5 major surgical complications: renal vein thrombosis in 2 cases, which led to graft loss, wound dehiscence in 2 cases and lymphocele in one case. One patient died of heart failure 6 months after transplantation, with a functioning graft. DGF occurred in $32 \%$ of cases and lasted a mean $6.1 \pm 5.4$ days. The incidence of acute rejection was $16.9 \%$. Renal function at 1 and 2 years was excellent with serum creatinine levels of $121 \pm 35$ and $126 \pm 31 \mu \mathrm{mol} / \mathrm{L}$, respectively, considering the donor's age.

Conclusion: MPDKT form elderly donors allows a shorter operating time with few surgical complications, leaving the controlateral iliac fossa intact and giving elderly recipients a better chance of being transplanted.

\section{O071}

BILATERAL DISSECTION OF THE UPPER MEDIASTINAL LYMPH NODES ALONG THE RECURRENT LARYNGEAL NERVES DURING THORACOSCOPIC ESOPHAGECTOMY FOR ESOPHAGEAL CANCER 
G. Watanabe ${ }^{1}$, A. Itami, M. Kondo, A. Nomura, S. Hasegawa, H. Okabe, J. Kawamura, S. Nagayama, H. Inoue, S. Sato, Y. Sakai

1 Kyoto University, Kyoto, Japan

Background: Esophageal cancer has a high risk of widely spreading to lymph nodes. One of the most common sites of lymph node metastasis in intrathoracic esophageal cancer is the upper mediastinal region along both recurrent laryngeal nerves, occurring in $30-50 \%$ of all esophageal cancers. Some studies have emphasized the importance of lymph node dissection in this region. Recently, thoracoscopic esophagectomy has been reported, but standard techniques remain to be established for lymph node dissection in the upper mediastinal region. We describe our methods for lymph node dissection, with emphasis on identification of the left recurrent laryngeal nerve and on bilateral nodal dissection along the nerves during thoracoscopic esophagectomy with five ports. Anesthesia, body position, and thoracoscopic system. A double-lumen endotracheal tube is used to ventilate the left lung. The procedure is performed with the patient in the left decubitus position, with the surgeon facing the patient's back. A flexible scope is used, and monitors are placed cephalic to the patient. Port placement A five-port technique is employed. A scope port is placed at the midaxillary line in the seventh intercostal space. Two ports for the surgeon are placed at the posterior axillary line in the fifth and seventh intercostal spaces. Two ports are placed for the assistant, one at the midaxillary line in the third intercostal space and the other at the anterior axillary line in the fourth intercostal space. Operative procedure First, the arch of the azygos vein is ligated and cut with a surgical stapler, and the upper mediastinal pleura is incised along the right vagal nerve in a cephalic direction. On blunt dissection, the right recurrent laryngeal nerve is detected. This nerve branches from the vagal nerve and extends superiorly in an anteroposterior direction around the right subclavian artery. Lymph nodes are usually located dorsal to the right recurrent laryngeal nerve and can be dissected. The left recurrent laryngeal nerve is situated on the left side of the trachea and usually cannot be identified on a right thoracic view. The right side of the upper esophagus is mobilized from the trachea (the left portion of the esophagus is left intact). The esophagus is then retracted dorsally by means of a tape applied around the esophagus, drawing tissues on the left side of the trachea, including the left recurrent laryngeal nerve and lymph nodes, into the operative field. The left recurrent laryngeal nerve is easily detected, and lymph node dissection can be performed without direct retraction of the nerve, thereby reducing the risk of nerve injury.

Results and conclusion: Among 28 patients undergoing this procedure, about $37 \%$ had recurrent laryngeal nerve injury; most cases were transient. Our results suggest that thoracoscopic esophagectomy is technically feasible in the upper mediastinal region. The use of magnification with endosurgery may further enhance safety and outcomes.

\section{O072}

\section{ANTIBIOTIC PROPHYLAXIS IN SEVERE ACUTE PANCREATITIS:} EXPERIMENTAL EVALUATION OF ERTAPENEM EFFICACY

F. Catena' ${ }^{1}$ F. Catena ${ }^{2}$, F. Gazzotti' ${ }^{2}$ L. Ansaloni², S. Di Saverio², S. Gagliardi², A. Amaduzzi' ${ }^{2}$ L. D'Alessandro' ${ }^{2}$ A.D. Pinna ${ }^{2}$ Bologna, Italy 2St Orsola- Malpighi University Hospital, Bologna, Italy

Objective: Secondary infection of the inflamed pancreas is the principal cause of death after severe acute pancreatitis (AP).

Prophylactic antibiotics that were used in AP were always initiated after hours from induction of pancreatitis. The effectiveness of antibiotics initiated earlier is unknown. The aim of this study was to evaluate the effectiveness of ertapenem initiated when AP is induced in rats. Method: 40 Sprague-Dawley rats were studied. AP was induced in rats by intraductal injection of $3 \%$ taurocholate. Rats were divided randomly into two groups: group 1 rats received normal saline as a placebo, group 2 received ertapenem $15 \mathrm{mg} / \mathrm{kg}$ after AP induction. At $24 \mathrm{~h}, 20$ rats (10 group I and 10 group 2) were killed for quantitative bacteriologic study. A point-scoring system of histological features was used to evaluate the severity of pancreatitis. The remaining 20 rats (10 group I and 10 group 2) were killed after 21 days for quantitative bacteriologic study and survival analysis. Chi square, Student and Kaplan- Meier tests were used for survival analysis. Results: All rats showed pathologic signs of acute pancreatitis. Ertapenem administrated after induction of AP significantly reduced the prevalence of pancreatic infection and 24 hours mortality as compared to controls. 21 days survival was significantly better in group 2 compared to group 1 . Conclusion: Early antibiotic prophylaxis with ertapenem reduces pancreatic infection rate after AP and it has a beneficial effect on survival.

O073

\section{EFFECTS OF ACUTE FENTHION TOXICITY ON LIVER AND KIDNEY} FUNCTION AND HISTOLOGY IN RATS

O. Sakrak ${ }^{1}$, M. Kerem¹, N. Bedirli², N. Gurbuz¹, O. Ekinci' ${ }^{1}$ A. Bedirli',

H. Pasaoglu ${ }^{1}$

1 Gazi University, Ankara, Turkey

2 Diskapi Training and Research Hospital, Ankara, Turkey

Background: Organophosphate (OP) compounds excert their biological effects through the blockade of acetylcholinesterase (AChE) enzyme. Fenthion is a systemic OP insecticide widely used in both agricultural and urban areas. The aim of this study is to investigate the dose related effects of fenthion on liver and kidney and the correlation between severity of organ damage and serum butyrylcholinesterase (BChE) enzyme levels. Methods: Ninety male Wistar rats were used in this study The animals were subdivided into control group and study group. Control group was administred $1 \mathrm{ml} 0.09 \% \mathrm{NaCl}$ intraperitoneally (i.p.). Study groups received $25,50,75$ and $100 \mathrm{mg} / \mathrm{kg}$ of fenthion, i.p. Blood samples, renal and hepatic tissues were collected $24 \mathrm{~h}$ after dosing. In a separate set of experiments, survival was monitored for 7 days after saline or fenthion administration.

Results: While there was no mortality in the control group, mortality is observed in two rats receiving $75 \mathrm{mg} / \mathrm{kg}$ fenthion and three rats receiving $100 \mathrm{mg} / \mathrm{kg}$ fenthion. The vast majority of lethality occurred within two days of experiment. There was a correlation between the fenthion dosage and the increase in the blood levels of liver enzyme, creatinine and BUN. Serum BChE enzyme levels in rats receiving 75 and $100 \mathrm{mg} / \mathrm{kg}$ fenthion were statistically lower comparing to rats receiving $25-50 \mathrm{mg} / \mathrm{kg}$ fenthion. In rats, $25-50 \mathrm{mg} / \mathrm{kg}$ fenthion group serum BChE levels were low but did not show meaningful differences with control group. Histopathologic examination of the liver and the kidney indicated a significant injury only in rats receiving $100 \mathrm{mg} / \mathrm{kg}$ fenthion Conclusion: An organophosphate fenthion, particularly at high dosage has a toxic effect on liver and kidney and its toxicity is corelated with BChE enzyme levels.

\section{O074}

\section{SOMATOSTATIN RECEPTOR SCINTIGRAPHY FOR RENAL CELL CARCINOMA METASTATIC TO THE PANCREAS}

T.E. Lans, C.H.J. van Eijck, D. Kwekkeboom, H. Obertop Erasmus Medical Center Rotterdam, Rotterdam, The Netherlands

Background: Isolated pancreatic metastases of clear cell renal carcinoma are rare usually asymptomatic and difficult to find. Since Somatostatin receptors (SS-R's) are present on most primary renal cell tumors. Somatostatin receptor scintigraphy (Octreoscan $R$ ) was used to localize metastatic spread to the pancreas.

Methods: Eight patients with renal cell carcinoma metastatic to the pancreas were traced from a single center institution. In five patients the Octreoscan was added in the preoperative work-up.

Results: Of eight patients five patients underwent resection with good prognosis. One patient underwent palliative therapy by ERCP with stenting and died within six months and one patient without therapy also died six months after the radiological diagnosis. One patient was treated for a simultaneous bowel disease that was more life threatening. The Octreoscan was positive in all five patients; three patients with a hot spot in the pancreatic head, one with a leasion in the pancreatic tail and in one patient extensive disease was found in the pancreatic region. Conclusion: The Octreoscan is able to detect asymptomatic renal cell metastases. It will be of use in the preoperative diagnostic work-up of patients with histologic unidentified tumors in the pancreas.

\section{O076}

\section{FUNCTIONAL CHANGES IN ARTICULAR CARTILAGE AFTER} MENISCAL ALLOGRAFT TRANSPLANTATION IN RABBITS

P.C. Rijk ${ }^{1}$, W. Tigchelaar-Gutter ${ }^{2}$, C.J.F. van Noorden ${ }^{2}$

1 Medical Center Leeuwarden, Leeuwarden, The Netherlands

2 Medical Center Amsterdam, Amsterdam, The Netherlands

Removal of the meniscus leads to progressive degenerative arthritis of the knee on a long-term basis. Therefore, meniscal allograft transplanta- 
tion has been proposed as an alternative to meniscectomy. The success of meniscal allograft transplantation must not be judged solely on the basis of incorporation of the transplant into the host knee but must be evaluated on the basis of protective effects of the transplant on the articular cartilage. The purpose of this study is to evaluate functional changes quantitatively in articular cartilage after meniscal allograft transplantation in rabbits. Thirty rabbits were divided into 5 groups. Group A and group C were subjected to meniscectomy. Group B and group D underwent meniscal transplantation immediately after meniscectomy. Group $\mathrm{E}$ had delayed transplantation at 6 weeks after meniscectomy. Six nonoperated knees served as controls. Functional changes in articular cartilage were examined at 6 weeks (Groups A, B) and 1 year (Groups C,D,E, controls) after surgery by measuring lactate dehydrogenase activity (LDH) in chondrocytes as a measure of their vitality and proteoglycan content of the extracellular matrix as a measure of its quality. At 6 weeks and 1 year follow-up, no significant differences were found between the immediate transplant group and the postmeniscectomy group. The delayed transplant group showed a signifantly decreased proteoglycan content as compared with the postmeniscectomy group at 1 year follow-up. No significant differences in cellular LDH activity were found between the immediate transplant group and postmeniscectomy group at 6 weeks and 1 year. However, the delayed transplant group showed a significant decrease in LDH activity as compared with the postmeniscectomy group. It can be concluded that immdiate meniscal transplantation in rabbits does not significantly reduce degenerative changes of articular cartilage in comparison with meniscectomy on a short term and long term basis, whereas delayed transplantation leads to even more degenerative changes than meniscectomy only. Before meniscal allograft transplantation can be considered as an alternative to meniscectomy in clinical practice, considerably more data are needed. However, from the present study it is clear that delayed meniscal transplantation has to be considered with great care.

\section{O077}

ACUTE MAJOR AIRWAY INJURIES: CLINICAL FEATURES AND MANAGEMENT, A FIVE YEAR EXPERIENCE FROM FOUR UNIVERSITY HOSPITAL

\section{S. Ayazi', M.B. Shadmehr ${ }^{2}$, K. Ayazi \\ 1 University Of Southern California, Los Angeles, Usa \\ 2 Surgery, Shaheed Beheshti University, Tehran, Iran}

Introduction: Airway trauma is rare but morbid. They are encountered with increasing frequency because of improvements in prehospital care and early initiation of the Advanced Trauma Life Support (ATLS). The study is aimed to give a description of patients with airway trauma, to help providing data for better emergency services \& delayed managements of this type of trauma.

Methods \& materials: This retrospective, cross sectional study was conducted in 4 university hospitals in Tehran, Iran; from 1997 to 2002 the study was conducted with 28 patients. Demographic data, mechanism of trauma, site of trauma, clinical manifestations, length of hospitalization, complications, mortality \& treatment outcome were the main subjects extracted from units. SPSS 10.01 was used for analysis.

Results: $22(78.6 \%)$ patients were males and $8(21.4 \%)$ were female.. Mean age was $33.1 \pm 2.2$ years. $8(28.5 \%)$ cases presented with blunt trauma, $19(67.8 \%)$ with penetrating trauma \& 1 had inhaled burning chemical agent. $22(78.6 \%)$ of cases had injuries in cervical airways $\&$ the rest $6(21.4 \%)$ in thoracic airways. subcutaneous emphysema 7 $(50 \%)$, dysphonia \& dyspnea each with incidence rate of $6(42.8 \%)$ were the most frequent manifestations. $85.7 \%$ of our patients underwent surgical treatment. Mean length of hospitalization was $5 \pm 2$ days. The total rate of incidence for complications was $14.2 \% ; 2(7.2 \%)$ cases of wound infection, $1(3.5 \%)$ with anastomosis dehiscence \& $1(3.5 \%)$ with collapsed lung after thoracotomy. The mortality rate was $10.7 \%$ ( 3 patients). The treatment outcome assessed excellent in $80 \%$ of cases, good in $1(3.8 \%)$, acceptable in $1(3.8 \%)$ \& poor in $3(11.5 \%)$.

Discussion: Young men were more at risk for airway trauma particularly the penetrating types. Lower mortality and favorable treatment outcome are outstanding in this study. Rate of complication is average. Studies on non surgical treatments \& autopsy based studies for airway trauma are suggested for complementary results.

O078

DEDICATED PLASTIC SURGERY TRAUMA THEATRE IMPROVES THE DELIVERY OF ACUTE PLASTIC EMERGENCY SERVICES IN THE NHS
F.C. Chan ${ }^{1}$, Lau' ${ }^{2}$ S. Huang ${ }^{2}$, Canal ${ }^{2}$, Grant ${ }^{2}$, Cormack $^{2}$

1 Addenbrooke University, Dublin, Ireland

2 Addenbrookes University Hospital, Cambridge, United Kingdom

Introduction: There is a perceived delayed in treating acute plastic surgery emergencies in the NHS across the UK. This study examined the efficacy of emergency plastic patient service in plastic surgical units with or without dedicated plastic surgery trauma theatre (DPSST).

Methods: Postal questionnaires were sent to 48 acute plastic surgical units in the NHS across the UK. 35/48 (72.9\%) replies were received. Results: Those units with dedicated trauma theatre $(n=18)$ reported significant improvement in the delivery of plastic emergency services, patients satisfaction rate and reduced 'injury to theatre time (ITT)' $(p<$ $0.05)$. However, patient attended plastic surgical units without DPSST $(n=17)$ often suffered numerous cancellations $($ mean $=3)$ and have a mean 'injury to theatre time' of 32.1 hours $(p<0.05)$. These units reported that DPSTT will potentially improve the delivery of plastic emergency services, patients' satisfaction rate, standard of emergency surgical training for trainees and also reduce hospital stay and cost $(p<0.05)$. Conclusion: Delays in treating emergency surgery cases affects patients' outcome. Dedicated plastic surgery trauma theatre can improve the delivery of acute plastic emergency services in those plastic surgical units without DPSST in the NHS.

\section{O079}

\section{CELLULAR CHANGES IN SPLEEN OF TRAUMA PATIENTS}

W.L. Olszewski, A. Wluka, M. Zaleska, M. Durlik Medical Research Center, Warsaw, Poland

Trauma and infections activate human immune system. Its components as spleen, lymph nodes, bone marrow and circulating immune cells participate in elimination of microbial and self-antigens derived from damaged tissues. Therapy with fluid infusions, administration of antibiotics and pressure regulating drugs as well as and plasma transfusions further affect the function of immune system. Most studies on the response of immune defense system to trauma have been devoted to the function of blood cells. The knowledge on the response of spleen and other lymphoid organs remains rudimentary. Spleen is a reservoir of erythrocytes, B and NK cells and acts as a scavenger organ for degraded cellular components and circulating microbes. Lack of spleen brings about certain types of immunodeficiency. It would be important to know how does the spleen react to mechanical trauma of large body mass and the resuscitating procedures.

Aim: To study the cellular composition of the functional areas of human spleen in trauma patients undergoing prolonged resuscitation.

Materials and methods: Twenty spleen specimens from trauma patients, unsuccessfully resuscitated, serving as organ donors were used. Patients received routine treatment including fluid replacement, antibiotics, and pressure-regulating drugs. Phenotypes of cells in various functional regions of spleen were identified using monoclonal antibodies to $T$ and $B$ lymphocytes, macrophages, migrating and follicular dendritic cells. Responsiveness to microbial antigens was studied in culture. Normal spleens obtained from patients with abdominal trauma and intraoperatively ruptured spleen served as controls.

Results: Immunohistochemical evaluation. Spleens were depleted of lymphocytes and erythrocytes. Cell density was 3-5-times less than in controls. The white pulp contained few CD3 (CD4 and CD8) cells around the arterioles. There were few CD123 dendritic cells.

The CD34 expression on endothelial cells was downregulated.

A number of dendritic-like cells contained cellular debris. Red pulp had few erythrocytes. The responsiveness of splenocytes to E.coli, LPS and $\mathrm{CpG}$ in a $24 \mathrm{~h}$ culture was 5 -times less than in controls. Staph. epidermis did not stimulate splenocytes. Only a few bacteria were phagocytyzed. Conclusion: Trauma patients in terminal stages reveal depletion of splenocytes and their low responsiveness. This observation may partly explain the mechanism of immune deficiency in traumatized patients and point to the necessity of mobilizing the stores of immune cells (BMC?)

\section{O080}

HAMMERING KIRSCHNER WIRES: INSERTION TECHNIQUE OF THE NEAR FUTURE?

B.B.G.M. Franssen, A.H. Schuurman, M. Kon

University Medical Center Utrecht, Utrecht, The Netherlands 
Background: Heat generation due to drilling Kirschner wires (K-wires) into bone can lead to osteonecrosis and loosening of K-wires. Hammering K-wires could be an alternative insertion method. It is known that this technique generates less heat, results in better fixation and a shorter insertion time. There is however, no information about heat generation, extraction force and insertion time of hammered K-wires using a human in vitro model. Therefore these variables were compared in this study.

Materials and methods: Temperature, insertion time and extraction force were compared between three K-wire insertion methods ' drilling without irrigation, drilling with irrigation and hammering. Forty-five $\mathrm{K}$-wires were inserted into 15 fresh human cadaver metacarpalia with thermocouples placed 0.5 and $1.0 \mathrm{~mm}$ from the periphery of the insertion place at a depth of $1.0 \mathrm{~mm}$. All three insertion methods were applied in each metacarpal. Drilling was accomplished at a constant $1.0-\mathrm{kg}$ load and drill speed of $1,200 \mathrm{rpm}$. External irrigation at $10 \mathrm{~mL} / \mathrm{min}$ with normal saline was used. Hammering was accomplished at a constant 0.5$\mathrm{kg}$ load with 10,000 pulses per minute. Extraction forces were measured directly after insertion

Results: Average values for maximum recorded temperatures, insertion times and K-wire fixation for each insertion method were as follows: drilling without irrigation, drilling with irrigation and hammering gave maximum temperatures of $88.3 \pm 21.2^{\circ} \mathrm{C}, 26.2 \pm 2.5^{\circ} \mathrm{C}$ and $52.9 \pm 12.1^{\circ} \mathrm{C}$ respectively. The insertion times were $263.2 \pm 141.5 \mathrm{~s}, 196.1 \pm 110.5 \mathrm{~s}$ and $49.9 \pm 32.1 \mathrm{~s}$ for drilling without irrigation, drilling with irrigation and hammering, respectively. Extraction forces needed for drilling without irrigation, drilling with irrigation and hammering were $39.9 \pm 16.0 \mathrm{~N}$, $57.8 \pm 25.1 \mathrm{~N}$ and $59.3 \pm 22.7 \mathrm{~N}$, respectively.

Conclusion: Hammering K-wires results in a shorter insertion time, lower maximum temperature and higher extraction forces compared to drilling without irrigation. The only advantage of drilling with irrigation over hammering is a lower temperature.

\section{O081}

\section{IS IL6 GENE PROMOTER POLYMORPHISMS RESPONSIBLE FOR DISTURBANCES IN FRACTURE REPAIR?}

G. Szczesny, W.L. Olszewski, B. Interewicz, E. Stachyra, J. Rutkowska Medical Research Center, Warsaw, Poland

Despite improvement in operative technique, reduction of bacterial infections and improved pharmacotherapy, non-healing of fractures affects about $10-15 \%$ of patients. Genetic factors are reported to predispose to the delayed healing. The aim of study was to search for genetic factors predisposing to disturbances in bone union.

Patients \& methods: Venous blood specimens were harvested from patients $(n=27)$ treated due to disturbances in bone fracture repair (malunions, non-unions), diagnosed clinically and by standard X-ray and CT scans. DNA was isolated from blood cells for determination of polymorphism of IL-6 (G-174C), CRP(G1059C) and CD14(C-159T) genes IL-6 5'-TGACTTCAGCTTTACTTTGT -3', 5'-CTGATTGGAACCTTATTAAG-3', CRP F 5' GATCTGTGTGATCTGAGAAACCTCT3', R 5' GAGGTACCAGAGACAGAGACGTG3';CD14 f 5' GTGCCAACAGATGAGGTTCA 3', r 5' CGCAGCGGAAATCTTCATC 3'. The obtained results were compared with those from 110 healthy blood donors without disturbances in wound healing or predisposition to infection in the past history. Results. The obtained data showed significant differences in the frequency of mutations in IL6 in the patients group (CC15\%, and GC37\%; whereas GG48\%) when compared with controls (CC4\%, GC 0\%, whereas GG96\%). There were no significant differences in CRP and CD14 allele representation. Discussion. Our observations points to the possibility of genetic predisposition to fracture non-healing in IL-6 gene. IL-6 is a proinflammatory cytokine participating in the activation of the immune response against infection. Since the mutations in IL-6 gene promoter were reported to correlate with lower serum IL-6 concentration (Eur Cytokine Netw. 2006; 17: 181-8), our results suggest that this mutation may impair healing by predisposing to infection. This finding may be of interest, since our previously reported data showed the presence of bacteria using standard microbiological isolation technique in $24 \%$ and bacterial DNA in the callus specimens obtained from patients with delayed unions. Conclusions. High frequency of mutations in the IL6 gene correlates with non-healing of bone fracture.

\section{O082}

GRANULOCYTE-COLONY STIMULATING FACTOR (G-CSF) ENHANCES MUSCLE PROLIFERATION AND STRENGTH FOLLOWING SKELETAL MUSCLE INJURY IN RATS
I. Stratos, R. Rotter, C. Eipel, T. MittImeier, B. Vollmar University of Rostock, Rostock, Germany

Insufficiency of skeletal muscle regeneration often impedes the healing process with functional deficiencies and scar formation. We therefore tested the hematopoietic growth factor G-CSF with respect to its efficacy to improve functional muscle regeneration following skeletal muscle injury in Wistar rats. After standardized open crush injury to the left soleus muscle, animals received daily G-CSF $(20 \mu \mathrm{g} / \mathrm{kg}$ ip) or vehicle solution $(n=21$ per group). Sham-operated animals without muscle injury served as controls $(n=15)$. After in vivo assessment of the fast twitch and tetanic contraction capacity of the soleus muscles at days 4,7 and 14 post-injury, sampling of muscle tissue served for analysis of muscle tissue cell proliferation (bromodeoxyuridine (BrdU)-immunohistochemistry) and cell apoptosis (TUNEL-analysis). Muscle strength analysis revealed recovery of contraction forces to $28 \pm 2,35 \pm 2$, and $54 \pm 3 \%$ (twitch force) and to $21 \pm 3,25 \pm 2$ and $39 \pm 2 \%$ (tetanic force) within the $14 \mathrm{~d}$-observation period in vehicle-treated animals. In contrast, G-CSF increased contractile forces with significantly higher values at day 7 (twitch force: $41 \pm 1 \%$; tetanic force: $35 \pm 2 \%$ ). This enhancement of muscle function was preceded by a significant increase of cell proliferation (BrdU-positive cells/mm2: $30 \pm 4$ vs vehicle: $16 \pm 4$ ) and a significant decrease of cell apoptosis (TUNELpositive cells $/ \mathrm{mm} 2: 7 \pm 2$ vs. vehicle: $17 \pm 4$ ) at day 4 . At later time points groups did not further differ with respect to proliferation and apoptosis. In conclusion, G-CSF histologically promoted viability and proliferation of muscle cells and functionally enhanced recovery of muscle strength. Thus, G-CSF might represent a therapeutic option to optimize the posttraumatic course of muscle tissue healing.

\section{O083}

\section{GAIT ANALYSIS AFTER PERCUTANEOUS REPAIR OF DISPLACED INTRA-ARTICULAR CALCANEAL FRACTURES}

T. Schepers ${ }^{1}$, A. van der Stoep ${ }^{1}$, H. van der Avert $^{2}$, I.B. Schipper ${ }^{1}$, D. den Hartog ${ }^{1}$, L.M.M. Vogels ${ }^{1}$, E.M.M. van Lieshout ${ }^{1}$, P. Patka ${ }^{1}$ 1 Erasmus Medical Center Rotterdam, Rotterdam, The Netherlands 2 Jongenengel Orthopedic Center Rotterdam, Rotterdam, The Netherlands

Introduction: Clinical results of the treatment of displaced intra-articular calcaneal fractures are mainly assessed using disease-specific outcome scores, physical examination and radiographs. The aim of the current study was to analyze the gait pattern of patients with a unilateral displaced intra-articular calcaneal fracture treated according our modified percutaneous distraction and fixation technique. The second aim was to determine the clinical relevance of gait analysis by studying whether gait pattern parameters correlated with established outcome measurements such as disease specific questionnaires, fracture classification, radiographic data and physical exam data.

Patients and methods: Twenty-one patients with unilateral displaced intra-articular calcaneal fractures treated by percutaneous reduction and screw fixation participated in this study. The gait measurements of the injured foot were compared with the contralateral (control) foot. Intra- and interobserver reliability was calculated for all gait pattern parameters determined by calculating the interclass correlation coefficient (ICC). Correlations between the ratios (injured/control) of various gait pattern parameters and outcome scores, the physical exam items ratios, the fracture classification and the radiological parameters were determined.

Results: For all gait pattern items measured, the ICC ranged from 0.83 to 1.00 . With a median follow up time of 18 months, patients generally put more weight on the control foot than on the injured foot while standing. In addition, a statistically significantly reduced total contact time and higher maximum pressure below the first metatarsal was found for the injured foot as compared to the control foot. Of all correlations calculated, only the heel time ratio correlated statistically significant with the heel width ratio. There was no correlation between gait analysis and outcome scoring systems.

Conclusion: The high intra-observer and inter-observer agreement implies that the gait analysis as performed during this study is a highly reliable method. Significant differences in the gait pattern between the injured and control foot were found in patients with an intra-articular calcaneus fracture 18 months post-injury. Due to the poor correlation with outcome measures, gait analysis will not replace the traditional measures of functional outcome. Instead, as already suggested in literature, gait analysis might be a valuable addition in determining functional outcome after intra-articular calcaneal fracture treatment. 


\section{O084}

\section{HIGH-FAT NUTRITION AFTER HEMORRHAGIC SHOCK PREVENTS LOSS OF GUT BARRIER FUNCTION}

J.J.de Haan ${ }^{1}$, T. Lubbers ${ }^{1}$, M. Hadfoune ${ }^{1}$, M.D. Luyer ${ }^{2}$, C.H. Dejong ${ }^{2}$, W.A. Buurman', J.W. Greve ${ }^{2}$

1 University Hospital Maastricht, Maastricht, The Netherlands 2 University Hospital Maastricht, Maastricht, The Netherlands Considering the high morbidity and mortality related to SIRS and sepsis, interventions that reduce inflammation and organ damage following major operations and trauma are needed. Previously, we showed in a hemorrhagic shock model in rats that high-fat nutrition administered before shock reduces inflammation and preserves gut barrier function via a cholecystokinin (CCK)-dependent mechanism. However, in clinical situations such as trauma, shock is already present before treatment can be started. Therefore, the aim of this study is to determine the effects of high-fat feeding after shock on gut wall integrity and to explore the role of CCK in this setting. Shock was induced by male SpragueDawley rats via extraction of $30-40 \%$ of blood volume. Following shock, rats were either fasted or received a high-fat nutrition, containing high amounts of phospholipids, or an isocalorical low-fat feeding at 80, 180 and 360 minutes after shock. The involvement of CCK was assessed by administration of CCK-A and CCK-B receptor antagonists at 60 and 160 minutes after hemorrhage. At 24 hours following shock, gut barrier function was assessed by determination of bacterial translocation to mesenteric lymph nodes, spleen and liver and measurement of ileal permeability to horseradish peroxidase (HRP). Bacterial translocation was reduced by high-fat feeding $(40.4 \pm 4.0 \mathrm{CFU} / \mathrm{g})$ compared to low-fat $(68.6 \pm 4.9 \mathrm{CFU} / \mathrm{g} ; \mathrm{p}<0.01)$ and fasted groups $(133.1 \pm 16.8 \mathrm{CFU} / \mathrm{g}$; $\mathrm{p}<0.001)$. Administration of CCK receptor antagonists abrogated the protective effect of high-fat feeding $(p<0.001)$. High-fat nutrition also lowered permeability to HRP in comparison to low-fat and starved animals $(6.5 \pm 0.2$ vs. $7.1 \pm 0.3$ vs. $9.1 \pm 0.3 \mu \mathrm{g} / \mathrm{ml})$. This effect was blocked by administration of CCK receptor antagonists. In conclusion, high-fat nutrition administered after hemorrhagic shock preserved gut barrier function in a CCK-dependent manner. These findings show for the first time a protective effect of high-fat nutrition when given after shock. Accordingly, high-fat nutrition may be a potential new therapeutic option for trauma patients in which shock is already present.

\section{O085}

FOLEY CATHETER BALLOON TAMPONADE FOR LIFE THREATENING PENETRATING NECK INJURIES

\section{C.G. Troskie}

University of Cape Town, Groote Schuur, Cape Town, South Africa

Background: Foley catheter(FC)balloon tamponade is a well-recognized technique employed to arrest hemorrhage from penetrating wounds. The aim of this study is to review our experience with this technique in penetrating neck wounds and to propose a management algorithm for patients with succesfull FC tamponade.

Methods: A retrospective chart review (July 2004-June 2005 inclusive)was performed of patients identified from a prospectively collected penetrating neck injury computer database in whom a FC balloon tampande was used.The units policy for penetrating neck injuries is one of selective non-operative management. All patients with successful FC tamponade underwent angiography. A venous injury was diagnosed if angiography was normal.Ancillary tests were performed as indicated. Removal of the FC was performed in the operating theatre.

Results: During the study period, 220 patients with penetrating neck injuries were seen. FC balloon tamponade was used in 18 patients. It was successfull in 17 patients Angiography was positive in 3 patients,all of whom underwent surgery. The FC was successfully removed in 13 patients at a mean of 72(range48-96) hours. One patient bled on removing the catheter, mandating emergency surgery.

Conclusion: Foley catheter balloon tamponade remains a useful adjunct in the management of selective patients with penetrating,bleeding neck wounds.

O086

POTENTIAL MECHANISMS FOR THE CHEMOPROTECTIVE EFFECT OF CRUCIFEROUS VEGETABLES IN COLORECTAL CANCER: A CLINICAL TRIAL OF INDOLE-3-CARBINOL

Mc. Grath, H. Frydoonfar, A.D. Spigelman

University of Newcastle, Newcastle, Australia

Aims: Despite the well recognised protective effect against various human cancers, including colorectal, of cruciferous vegetables little is known of how this effect is conferred. It is thought that some phytochemicals only found in these vegetables confer the protection. These compounds include the glucosinolates of which Indole-3-carbinol is one. They are known to induce carcinogen metabolising enzymes like the Phase 2 enzymes of the Glutathione transferase (GST) family. The other effects in humans are not well documented. We wished to assess the effect of Indole-3-carbinol on GST enzymes and the effect of treated human serum on two colon cancer cell lines in a similar fashion to our previous laboratory work.

Materials and methods: We carried out a double blind, placebo-controlled human volunteer study. All patients were given $400 \mathrm{mg}$ daily of Indole-3-carbinol for 3 months, followed by placebo. Serum samples were tested for GSTM1 genotype by Polymerase Chain Reaction. Serum GST levels were assessed using ELISA and Western Blot methodologies. Serum was exposed to HT29 and HCT116 colon cancer cells. Results: Forty nine volunteers completed the study. GSTM1 genotypes were obtained for all but 2 volunteers. A slightly greater proportion of volunteers were GSTM1 positive, in keeping with the general population. GST was detected in all patients. Total GST level was not affected by Indole-3-carbinol dosing against placebo. Although not statistically significant GSTM1 genotype affected the serum GST level in response to Indole-3-carbinol. There was a statistically significant decrease in proliferation of both cancer cell lines when exposed to serum during the Indole-3-carbinol treatment period against the baseline $(P=0.03$ +0.002 respectively). This difference was not seen when comparing against the placebo period.

Conclusion: Indole-3-carbinol does not alter the total serum GST leve during prolonged dosing. Serum from volunteers during the Indole-3carbinol dosing period caused inhibition of proliferation in colon cancer cells against the baseline.

O087

SURGICALLY-INDUCED BILE DUCT INJURY IS FOLLOWED BY EARLY LIVER DAMAGE. AN EXPERIMENTAL STUDY IN RATS

G. Lissidini, P. Portincasa, G. Piccinni, A. Gurrado, E. Poli, M. Testini University of Bari, Bari, Italy

GSH - LIVER CYTOSOL

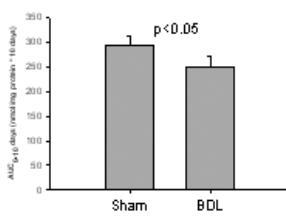

PSH. LIVER CYTOSOL

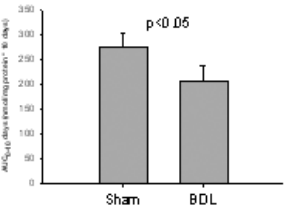

GSH and PSH in liver cytosolol GSH. LNER M TOCHONDRIR PSH - LIVER MITOCHONDRLQ
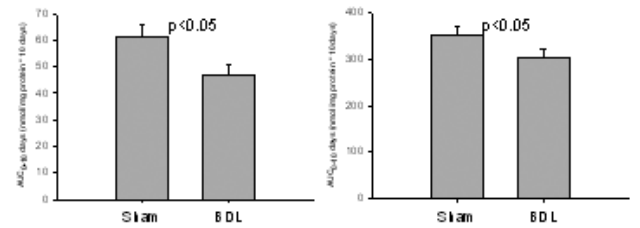

GSH and PSH in liver mytochondria

Introduction: Bile duct injury (BDI) resulting in post-hepatic obstruction continues to be the most serious complication of LC. Early detection and treatment of BDI is very important to prevent extrahepatic cholestasis that may result in liver failure. Aim of this study is to investigate if oxidative stress can be early detected in experimental model of short-term cholestasis induced by bile duct ligation (BDL).

Materials and methods: Adult male Wistar rats underwent surgery under general anesthesia; the common bile duct was identified, isolated, ligated and divided. Controls rats underwent a sham operation in which the common bile duct was exposed but not ligated. Animals were fasted overnight and sacrificed by decapitation at days 0 (baseline, control), 
$1,3,7$ and 10 after BDL. In each arm 15 rats were employed $(n=3$ at each time point). Livers were removed for morphological studies and biochemical analysis. The histologic features examined blindly by two pathologists unaware of the experimental protocol, were distorsion of bile ducts, biliary hyperplasia and neoductular genesis, cell necrotic and apoptotic features.

Results: Body weight was $300+/-15 \mathrm{~g}$ at baseline and increased to $310+/-20$ and $320+/-21 \mathrm{~g}$ in BDL and sham animals at day 10 , respectively $(p=N S)$. The baseline liver weight of $13.5+/-1 \mathrm{~g}$ increased to $16.5+/-$ $1.5 \mathrm{~g}$ in $\mathrm{BDL}(\mathrm{p}<0.01)$ at day 10 and remained unchanged in sham animals. Such modification was paralleled by the increased of serum ALT (from 85+/-33 UI/L to 309+/-25 UI/L, $p<0.001$ ) and serum alkaline phosphatase, from $154+/-29 \mathrm{UI} / \mathrm{L}$ to $429+/-32 \mathrm{UI} / \mathrm{L}$ ( $p<0.001$ ), which was not the case in sham animals. While there were no major changes in liver tissue in the control group, an early and progressive biliary ductular hyperplasia and neoductulo genesis, periductular inflammation, focal hepatocellular necrosis, porto-portal septa and ground glass/fathery degeneration of centrilobular area were observed in the BDL group. Cholestatic livers also showed an early (from day 3 ) and progressive decrease of cytosolic concentrations of GSH and PSH compared with the control group which remained unchanged. As a consequence, the estimated area-under-curve (AUC 0-10 days) of both parameters were also significantly decreased, compared to control values (figure 1). Besides, in mitochondria there was decreased GSH and concentrations with ongoing cholestasis, resulting in significantly decrease of AUC (figure 2) Conclusion: The results of this study suggest a new approach in the patients with certain bile duct injury. Besides, according to standardized approaches, up to now surgeons preferred to delay the biliary repair until the fourth week, when a biliary stricture with enlargement of proximal bile duct is established and after adequate assessment and delineation of the biliary tract to perform a more comfortable anastomoses between biliary tree and intestine. On the contrary, we emphasize a postoperative immediate repair of iatrogenic bile duct injury to prevent liver failure. This trend, in spite of a more difficult re-surgery due to a fine biliary tract, could ensure an appropriate treatment with an optimal patient outcome.

\section{O088}

\section{THE ROLE OF KUPFFER CELLS AND PLATELETS AFTER THE} ISCHEMIA-REPERFUSION OF THE LIVER -IN VIVO STUDY-

Y.N. Yoritaka Nakano, T.K. Kondo, R.M. Matsuo, S.M. Murata, N.O. Ohkohchi

University of Tsukuba, Tsukuba, Japan

Background: At the time of the liver surgery, the ischemia reperfusion $(\mathrm{I} / \mathrm{R})$ injury occasionally leads to the liver failure through activating the Kupffer cells (KCs) and the leukocytes. In addition, several articles have reported the role of platelets after I/R. In general, platelets have been considered to play an important role in hemostasis, but, recent studies suggest that platelets are associated with initiation and modulation of inflammatory responses. However, there are few reports demonstrating the relationship between the platelets-endothelium interaction and KCs in vivo after hepatic $I / R$

Aim: The aim of this study was to investigate the platelet-endothelium interaction in the liver sinusoids after hepatic I/R and the contribution of KCs to the adherent platelets using the intravital fluorescence microscopy (IVM). Method: Male Wistar rats, weighing 220 250g, were divided into three groups: A) no-ischemia group, in which animals were not performed hepatic ischemia ; B) I/R KC+ group, in which animals received 20 min of normothermic hepatic ischemia ; C) I/R KC- group, in which animals were injected clodronate-encapsulations liposome systemically for deletion of KCs before hepatic ischemia. At 30, 60, 120 min after reperfusion, rhodamine $6 \mathrm{G}$ labeled platelets were injected and these platelets were monitored by IVM system. The blood pressure was monitored through the experiments. Using the computer system, the recorded images were analyzed for estimating the microcirculatory parameters as follows : the number of the adherent platelets, the distribution of the platelets, and the microvascular diameter. Serum alanine aminotransferase (ALT), the number of platelets, and white blood cells were measured until $120 \mathrm{~min}$ after reperfusion.

Results: In the KC+ group, the number of adherent platelets significantly increased at $30 \mathrm{~min}$ after reperfusion $(P<0.05$ v.s. no-ischemia). The number of the leukocyte increased at $60 \mathrm{~min}$, and then the number of adherent platelets increased in proportion to the reperfusion time. On the other hand, in the KC- group, the number of adherent platelets did not increased until the 60 min after reperfusion. Compared with the $\mathrm{KC}+$ group, the number of adherent platelets significantly decreased $(P<0.01$ v. S. KC+). Liver enzymes (ALT) significantly decreased in the KC- group $(P<0.05$ v. s. $K C+)$. Compared with the diameter of hepatic central vein, the diameter of sinusoid was significantly contracted in the KC+ group. Conclusion: The endothelial adhesion of leukocytes was subsequent to the adhesion of platelets. The number of adherent platelets increased in proportion to the duration of reperfusion up to $120 \mathrm{~min}$. The deletion of KCs induced the decrease of adherent platelets and reduced hepatic injury after I/R. Therefore, the correlation between platelets and KCs might play an important role of the injury after hepatic l/R.

\section{O089}

INFLUENCE OF NEOADJUVANT CHEMOTHERAPY ON LIVER REGENERATION AFTER EXTENDED RESECTION WITH OR WITHOUT VASCULAR OCCLUSION

S. Manekeller ${ }^{1}$, V. Dobberahn ${ }^{1}$, A. Hirner ${ }^{1}$, T. Minor $^{1}$

1 Division of Surgical Research, Bonn, Germany, Surgical University

Clinic, Bonn, Germany

Introduction: The increasing interest in neoadjuvant chemotherapy of liver metastasis after colorectal carcinoma prior to resection has focussed surgical concerns to the influence of oncologic chemotherapy on hepatic tolerance to intraoperative ischemia as produced by Pringle's manoeuver. Hence, little to nothing is known about the livers regenerative capacity after preceding neoadjuvant chemotherapy. Hepatic viability and regeneration should thus be investigated after extended liver resection, depending on concomitant ischemia (Pringle) and systemic Chemotherapy.

Methods: Male Wistar rats (250-300g) were randomised in 2 groups. One group $(\mathrm{CH})$ received an intraperitoneal chemotherapy comprising $5 \mathrm{FU}(1000 \mathrm{mg} / \mathrm{m} 2)$, FA $(200 \mathrm{mg} / \mathrm{m} 2)$ und Oxaliplatin $(85 \mathrm{mg} / \mathrm{m} 2)$ while the placebo group $(\mathrm{PL}$ ) was given only saline according to the same proto$\mathrm{col}$. On the next day, all animals underwent a standardized $2 / 3$ resection of the liver (left-lateral and median lobe) with or without accompanying vascular exclusion by Pringle's manoeuvre (30min). Tissue samples and serum was analysed after $1 \mathrm{~h}, 24 \mathrm{~h}$ or 7 days after the operation.

Results: Chemotherapy did not entail any significant alterations of liver enzymes in systemic circulation compared to the placebo group prior to the operation. $24 \mathrm{~h}$ after liver resection, the rise in serum-activities was significantly attenuated in $\mathrm{CH}$ compared to $\mathrm{PL}$ by $50 \%, 30 \%$ and $60 \%$ for ALT, AST and GLDH, resp.; $p<0.05$ ). By contrast, bile production was found marginally reduced in $\mathrm{CH}(530 \pm 60 \mu \mathrm{l} / \mathrm{h}$ vs. $680 \pm 58)$.

However after 7 days all values were in the normal range in both groups. $24 \mathrm{~h}$ after resection, liver regeneration was sensibly slowed down in $\mathrm{CH}$ as evidenced by protein expression of Ki67 and PCNA (western blot and histochemistry) as well as the appearance of mitotic patterns.

After 7 days, however, liver mass gain was comparable in both groups. Most surprisingly, the combination of Pringle and liver resection led to the decease of 5 out of 6 animals in PL during the first 24h, but in $\mathrm{CH}$ $4 / 6$ animals survived for 7 days. Similarly, liver cell apoptosis (caspase 3 activation) was found induced after Pringle in PL but to a much lesser extend in $\mathrm{CH}$. One hour after Pringle, cell-protective chaperones (BIP/GRP78) were found induced (mRNA and Protein) in $\mathrm{CH}$ but not in $\mathrm{PL}$ and hyperphosphorylation of the eukariotic initiation factor elF2-alpha suggests adaptive stress responses at the endoplasmic reticulum known as the unfolded protein response in $\mathrm{CH}$ but not in $\mathrm{PL}$.

Conclusion: It is concluded that neoadjuvant chemotherapy actually does retard liver regeneration after resection but does not preclude long term recovery. $\mathrm{CH}$ must not be considered as risk factor regarding ischemic challenge to the liver, which might become necessary during surgery. By contrast, $\mathrm{CH}$ seems to confer a sort of preconditioning at the endoplasmic reticulum, which, by induction of antiapoptotic chaperones, increases the hepatic tolerance against liver resection under ischemic conditions

\section{O090}

HEPATIC ENERGY METABOLISM AND DIFFERENTIAL PROTECTIVE EFFECTS OF SEVOFLURANE AND ISOFLURANE ANESTHESIA IN RAT HEPATIC ISCHEMIA-REPERFUSION INJURY MODEL

N. Bedirli', E. Ofluoglu' ${ }^{2}$, M. Kerem², G. Utebey ${ }^{1}$, M. Alper ${ }^{1}$, A. Bedirli², O. Ozlu', D. Yilmazer ${ }^{1}$, H. Pasaoglu ${ }^{2}$

1 Diskapi Training and Research Hospital, Ankara, Turkey

2 Gazi University, Ankara, Turkey 
Background: Volatile anesthetics is known to protect the liver from ischemia-reperfusion (IR), however, the mechanism of cytoprotection is not well understood. This study investigates the effect of isoflurane and sevoflurane in a warm liver IR model on cytokines, hepatic tissue blod flow (HTBF), energy content and liver structure.

Method: Seventy-two wistar rats were randomly assigned into one of 3 groups: Control group $(n=24)$; no volatiles, sevoflurane group $(n=24)$; $2 \%$ sevoflurane, isoflurane group $(n=24) ; 1.5 \%$ isoflurane. Thirty min after the start of volatile anesthetics rats were subjected to $45 \mathrm{~min}$ hepatic ischemia and 2 and $4 \mathrm{~h}$ of reperfusion. From each group; eight rats were sacrified at the end of ischemia, 2 and $4 \mathrm{~h}$ of reperfusion. Aspartate aminotransferase (AST) and alanine aminotransferase (ALT), HTBF, malondialdehyde (MDA), TNF-a, IL-1 $\beta$, energy charge and histologic examination were used to evaluate the extend of liver injury.

Results: Serum ALT and AST levels were similiar in control and isoflurane groups while there was a significant decrease in sevoflurane group at post-ischemic period $(\mathrm{P}<0.01)$. HTBF was remarkably better in sevoflurane group than isoflurane group. Tissue MDA levels were significantly low in sevoflurane group comparing to isoflurane group at the $2 \mathrm{~h}$ of reperfusion $(P<0.05)$. After ischemia, 2 and $4 \mathrm{~h}$ of reperfusion TNF- $a$ and IL-1 $\beta$ values were low in sevoflurane group but it was not statistically important $(P>0.05)$. In sevoflurane group, hepatic ATP and energy levels were significantly high at all measurment times. The degree of hepatocyte injury was low in sevoflurane group.

Conclusion: The major finding of the current study is that volatile anesthetics showed differential protection from hepatic IR injury in rats. Clinically relavant concentrations of sevoflurane given before, during and after hepatic ischemia protected the liver against IR injury. Whereas the effects of isoflurane on hepatic IR injury was not notable.

\section{O091}

\section{EFFECT OF NUTRITIONAL SUPPLEMENTATION WITH 3-RICH POLYUNSATURATED FATTY ACIDS ON A HEPATIC WARM ISCHE- MIA/REPERFUSION INJURY IN RATS}

W.I. Iwasaki', M.K. Kume'2, K.K. Kudo'2, Y.O. Ono ${ }^{2}$, I.K. Kikuchi², M.Y. Yoshioka ${ }^{2}$, T.S. Sato ${ }^{2}$, Y.Y. Yamamoto ${ }^{2}$

1 Akita University School of Medicine, Akita, Japan

2 Department of Gastroenterological Surgery Akita U., Akita, Japan

Introduction: Ischemia/reperfusion (I/R) results in an inflammatory disorder which contributes to hepatic dysfunction. É polyunsaturated fatty acid (PUFA) is metabolized to 3 -series of prostaglandins that are less inflammatory in tissue reactions than the prostaglandins from É 6 PUFA An elevation of É 3/ É 6 ratio in preoperative nutrition might therefore suppress a warm I/R-induced liver injury. This study aimed to investigate whether the dietary supplementation of É 3 PUFA influenced the É 3 / $\dot{E}$ 6 ratio in the blood and liver tissues, and whether this supplementation ameliorated hepatic warm I/R injury in rats.

Methods: Male Wistar rats weighing $200 \pm 10 \mathrm{~g}$ were divided into two groups. In Group A, rats were orally supplemented with É 3-rich PUFA oil $(0.54 \mathrm{~g} /$ day, $4.86 \mathrm{kcal})$ besides normal chow for 7 days. This oil consisted of É 3 PUFA (Eicosapentaenoic acid (EPA) $119.2 \mathrm{mg} / \mathrm{g}$, Docosapentaenoic acid (DPA) $16.9 \mathrm{mg} / \mathrm{g}$, Docosahexaenoic acid (DHA) 79.5 $\mathrm{mg} / \mathrm{g}$ ) and É 6 PUFA (Linoleic acid (LA) $215.5 \mathrm{mg} / \mathrm{g}$, Arachidonic acid (AA) $4.6 \mathrm{mg} / \mathrm{g}$, Hono- $\gamma$-Linolenic acid $1.1 \mathrm{mg} / \mathrm{g}$ ). É $3 /$ É 6 ratio of this oil was 1:1. In Group B, rats were administered the same amount of water $(0.6 \mathrm{ml})$ for 7 days. After these treatments, concentrations of PUFA in the blood $(\mu \mathrm{g} / \mathrm{ml})$ and liver tissues ( $\mu \mathrm{g} / \mathrm{g}$ wet tissue weight) were quantified by gas chromatography (Group A: $n=7$, Group B: $n=6$ ). A 25-minute warm ischemia was produced by Pringle's maneuver (Group $A: n=8$, Group B: $n=7$ ). At 120 minutes of reperfusion, liver injury was evaluated by blood levels of AST (IU/I) and ALT (IU/I). Student t-test was applied to statistical analysis (mean \pm standard deviation).

Results: Concentrations of É 3 PUFA (EPA, DPA and DHA) in the blood and liver tissues were higher in group $A$ than in group $B$. EPA in the blood: A, $58.43 \pm 12.46^{*}$ vs. B, $31.67 \pm 6.41$; EPA in liver tissues: $A$, $880.07 \pm 115.13^{*}$ vs. B, $329.63 \pm 49.04$; DPA in the blood: $A, 48.14 \pm 12.35^{*}$

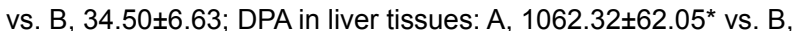
$710.63 \pm 103.00$; DHA in the blood: A, $82.43 \pm 14.82$ vs. B, $78.00 \pm 10.81$; DHA in liver tissues: A, 2508.11 $\pm 147.19^{*}$ vs. B, $2221.88 \pm 239.68$ (*: $p<0.05)$. É $3 / E$ E 6 ratio in group $A$ showed significantly higher value than that in group $B$ (In the blood: A, $0.43 \pm 0.05^{*}$ vs. $B, 0.25 \pm 0.02$; in liver tissues: $A, 0.55 \pm 0.05^{*}$ vs. $B, 0.35 \pm 0.03 ;{ }^{*}$ : $\left.p<0.05\right)$. At 120 minutes after reperfusion, serum release of AST and ALT was significantly suppressed in group A in comparison to group B (AST: A, 1129.9 $\pm 459.8^{*}$ vs. B,
1822.9 \pm 518.6 ; ALT: A, 1535.5 $\pm 621.2^{*}$ vs. B, $2311.4 \pm 635.1$; *: p<0.05). Conclusion: Preoperative 7-day supplementation with É 3 PUFA-rich diet effectively alleviated a warm I/R injury of rat livers by increasing É 3/ É 6 PUFA ratio in the blood and liver tissues.

\section{O092}

PORTAL BLOOD ARTERIALIZATION WITH A NEW EXTRACORPOREAL DEVICE TO TREAT TOXIC ACUTE LIVER FAILURE IN THE SWINE MODEL

B. Nardo', M. Tsivian², F. Neri², D. Prezzi², E .Bianchi², G.L. Piras², L. Puviani², R. Bertelli², V. Pacil' ${ }^{2}$, G. Cavallari², D. Cianciavicchia ${ }^{3}$ 1 Bologna, Italy

2 S. Orsola Hospital, University of Bologna, Bologna, Italy

3 Bellco, S.r.l., Modena, Italy

Background: The present study aimed to determine whether the portal blood arterialization by a new liver extracorporeal device (L.E.O.NARDO) is effective in treating swine with toxic administration leading to acute liver failure (ALF).

Methods: Eight swine with ALF induced by intraperitoneal injection of carbon tetrachloride in oil solution at a dose of $450 \mathrm{mg} / \mathrm{Kg}$ of body weight were randomly divided into two groups: four animals received L.E.O.NARDO treatment 24 hours after the intoxication. and four swine were not-treated (control group). Blood was withdrawn from the iliac artery and reversed in the portal venous system. An extracorporeal device was interposed between the outflow and the inflow in order to monitoring the hemodynamic parameters. Each treatment lasted 6 hours. Serum and liver samples were collected in both groups. The survival was assessed at 1 week.

Results: L.E.O.NARDO treatment yielded beneficial effects for carbon tetrachloride induced-ALF swine with decreased serum ammonia, transaminases and total bilirubin as compared with the untreated group. INR recovered rapidly in the L.E.O.NARDO group remaining significantly lower than in untreated animals. The 7-day survival of L.E.O.NARDO group swine was significantly higher than that of untreated animals, with a statistically significant difference $(p<0.05)$. Three swine in the L.E.O.NARDO group survived at 1 week while none of the swine in the control group were alive at that time.

Conclusion: Arterial blood supply in the portal system through the L.E.O.NARDO device is easily applicable, efficacious, safe and cost effective and may represent a novel approach for ALF swine induced by carbon tetrachloride intoxication.

\section{O093}

\section{DEPRIVATION OF HEPATIC STELLATE CELLS PREVENTS} ISCHEMIA-REPERFUSION INJURY OF THE LIVER IN RATS

T.T. Takahashi', M.Y. Yoshioka², S.S. Shibata ${ }^{2}$, H.U. Uchinami², Y.N. Nakagawa ${ }^{2}$, S.M. Motoyama ${ }^{2}$, T.S. Sato' ${ }^{2}$, Y.Y. Yamamoto ${ }^{2}$ 1 Akita university School of Medicine, Akita, Japan

2 Department of Gastroenterological Sug. Akita U., Akita, Japan

Introduction: Hepatic non-parenchymal cells are attributable to ischemia-reperfusion (I/R) injury of the liver. The participation of Kupffer cells and sinusoidal endothelial cells have been repeatedly documented. However, the contribution of hepatic stellate cells (HSCs) is not well studied. In this study, we examined whether the deprivation of HSCs affected the strength of I/R of the liver using gliotoxin that was known to induce apoptosis of HSCs in vitro.

Methods: Male Sprague-Dawley (SD) rats weighing 250-300g were used. The in vivo effect of gliotoxin pretreatment on the liver was examined in the gliotoxin-treated group and non-treated group. Gliotoxin was administered to rats intraperitoneally with a dose of $3.0 \mathrm{mg} / \mathrm{kg}$. The number of HSCs was studied by immunohistochemical staining of glial fibrillary acidic protein (GFAP), a marker of HSCs, on the formaldehydefixed, paraffin-embedded sections. The proportion of GFAP-positive cells to total cells in the liver was evaluated by measuring the stained areas in the randomly chosen five fields of the specimen using $\mathrm{NIH}$ Image. Changes in sinusoidal diameter were examined using intravital fluorescence microscopy. Total hepatic ischemia of 30 minutes was induced by Pringle's maneuver and followed by reperfusion of 6 hours. Serum levels of aspartate aminotransferase (AST) and alanine aminotransferase (ALT) were measured to evaluate the I/R injury of the liver in the treated group and the non-treated group. Student-t-test was used for a statistical analysis. Values were expressed as means \pm SD. 
Results: Immunohistochemical analysis demonstrated that gliotoxin treatment significantly decreased the percentage of GFAP-positive cells in the liver. The percentages of GFAP-positive cells (\%) were $1.91 \pm 0.46(n=8)$ in the treated group and $2.50 \pm 0.19(n=8)$ in the nontreated group. There was no difference in hepatic sinusoidal diameters between the treated and the non-treated groups in zones 1 and 2 of the liver. However, in zone 3, the sinusoids were wider in the treated group $(10.28 \pm 0.43 \mu \mathrm{m}, \mathrm{n}=3)$ than the non-treated group $(8.04 \pm 0.45 \mu \mathrm{m}, \mathrm{n}=3)$. Liver damage after I/R was significantly suppressed in the treated group $(P<0.01)$. The serum levels of AST and ALT (IU/L) were $298 \pm 148(n=9)$ and $121 \pm 50(n=9)$ in the treated group, respectively; and $515 \pm 160(n=9)$ and $350 \pm 134(n=9)$ in the non-treated group, respectively.

Conclusion: Treatment with gliotoxin significantly decreased the number of HSCs in vivo. Pretreatment with gliotoxin prevented I/R injury of the liver. Although a further study is necessary, dilation of the sinusoids in zone 3 might contribute to improving the microcirculation after I/R. It was suggested that HSCs also played a functional role in the I/R injury of the liver and their deprivation might be a novel strategy for ameliorating the liver damage after hepatobiliary surgery.

\section{O094}

ATTENUATION OF INFLAMMATION AND APOPTOSIS BY PRE- AND POST-TREATMENT OF DARBEPOETIN ALPHA IN ACUTE LIVER

\section{FAILURE OF MICE}

K. Le Minh, C. Eipel, K. Klemm, B. Vollmar

University of Rostock, Rostock, Germany

Introduction: In many liver disorders inflammation and apoptotic cell death are important pathogenic components, finally leading to acute liver failure. Haemotopoetic growth factors like erythropoietin are known to affect the interaction between apoptosis and inflammation in brain, kidney and myocardium. The present study aimed to determine whether pre- as well as posttreatment with erythropoietin might also exert hepatoprotection in a model of acute liver injury.

Material and methods: Male C57BL/6J mice were challenged with D-galactosamine (GalN, $720 \mathrm{mg} / \mathrm{kg}$ bw ip) and E. coli lipopolysaccharide (LPS, $10 \mu \mathrm{g} / \mathrm{kg}$ bw ip) and either pretreated (-24 h, GalN-LPS/DPOpre) or post-treated (+30 $\mathrm{min}$, GalN-LPS/DPOpost) with darbepoetin alpha (DPO, $10 \mu \mathrm{g} / \mathrm{kg}$ bw iv). Control mice received physiological saline (GalN-LPS/NaCl). Additional animals were treated with $0.9 \% \mathrm{NaCl}$ alone and served for assessment of physiological parameters $(\mathrm{NaCl})$. Six hours after application of GaIN/LPS or vehicle hepatic tissue damage and inflammatory response were analyzed by intravital fluorescence microscopy in ketamine/xylacine anaesthetized animals ( $n=7$ per group). After in vivo microscopy, animals were exsanguinated by puncture of the vena cava for immediate separation of EDTA plasma and liver tissue was asserved for subsequent Western blot analysis, histology and immunohistochemistry. Data are expressed as mean values \pm standard error of the mean. Statistical analysis was performed using ANOVA, followed by post-hoc-comparison ( ${ }^{*} p<0.05$ vs. $\mathrm{NaCl}, \# p<0.05$ vs. GalNLPS/NaCl).

Results: Administration of GalN-LPS provoked marked hepatic damage, characterized by an 3-fold increase of leukocyte adherence, nutritive perfusion failure of $\sim 40 \%$, an 12 -fold increase of hepatocellular apoptosis as well as enhanced enzyme release and necrotic cell death. DPO-pretreated, but also post-treated mice showed diminished intrahepatic leukocyte accumulation $(\mathrm{n} / \mathrm{mm} 2$; GalN-LPS/DPOpre: $201 \pm 2^{*}$, GalN-LPS/DPOpost: $130 \pm 30^{*}$ \#, GalN-LPS/NaCl: $263 \pm 36^{*}$, $\mathrm{NaCl}: 84 \pm 11$ ) and improved hepatic microcirculation (\% non-perfused sinusoids; $74.1 \pm 2.6^{*} \#, 76.8 \pm 3.5^{*} \#, 60.4 \pm 1.1^{*}, 98.2 \pm 0.2$ ). Hepatocellular apoptosis, as assessed by in vivo bisbenzimide staining, cleaved caspase-3 immunostaining and Western blot protein analysis, was significantly reduced by $50-75 \%$ after DPO pre- as well as post-treatment. There was unchanged protein levels of antiapoptotic Bcl-XL and proliferating cell nuclear antigen, but slightly decreased proapoptotic Bax protein. In addition, pre- and post-treatment with DPO significantly abrogated necrotic cell death (\% necrosis: $11 \pm 7^{\star} \#, 18 \pm 7^{*} \#, 50 \pm 10^{*}$, $0.7 \pm 0.5$ ) and liver enzyme release (U/I ALT: $92 \pm 24 \#, 145 \pm 76^{*}, 267 \pm 51^{*}$, $34 \pm 4$ ), implying the overall benefit of DPO on liver tissue integrity. Conclusion: Pre- but also post-treatment with DPO significantly lowers acute liver injury and augments morphologic recovery most probably through blockade of hepatocellular apoptosis. These observations may stimulate the evaluation of DPO, a compound established as clinically safe, as hepatoprotective therapy in patients with acute liver injury.

\section{O095 \\ RAPAMYCIN SUPPRESSES EXPRESSION OF GENES IMPORTANT FOR LIVER REGENERATION}

\section{Palmes}

Muenster University Hospital, Muenster, Germany

Introduction: The antineoplastic effect of Rapamycin (RAPA) has offered new therapeutic options for inhibition of hepatocellular carcinoma after orthotopic liver transplantation. However, the diverse effects of RAPA may also impair liver regeneration which becomes clinically important in the course of increased use of reduced-size liver. In this study a rat model of regenerating liver was used to gain insight into the temporal effects of RAPA to obtain an integrated view of different determinants critically important for reduced-size liver transplantation.

Materials and methods: 154 Lewis-rats were divided into 4 groups (I: sham-operation + RAPA p.o., II: sham-operation + vehicle, III: 2/3-liver resection + RAPA p.o., IV: 2/3-liver resection + vehicle). The expression of 22 genes important for inflammation, cell proliferation, extracellular matrix production, adhesion and angiogenesis was determined in liver by real-time RT-PCR analysis at early ( $24 \mathrm{~h}$ and $48 \mathrm{~h}$ ) and late (14 days) time points. Liver regeneration was assessed by intravital microscopy (hepatocytes, HSC, sinusoids) and immunohistochemistry (Ki-67, VEGF).

Results: Liver regeneration (hepatozytes/acinus day 4; III: $2148,7 \pm 28,2$ vs. IV: $2679,9 \pm 33,6, p<0.05$ ) and neo-angiogenesis (sinusoids/acinus day 3; III: $575,6 \pm 15,9$ vs. IV: $740,7 \pm 23,6, p<0.05$ ) were significantly decreased between the 3rd and 7th day after RAPA treatment, but normalized at day 14. Expression of growth factors (HGF, IGF and PDGFB$R$ ), and also of factors important for angiogenesis (VEGF, VEGF-R2, and angiotensin) was significantly down-regulated in rat liver treated with RAPA $(p<0.01)$ as compared to untreated control. Down-regulation of genes was observed at early time points, whereas gene expression was not affected by RAPA after the hepatocyte replication cycle during regeneration has been completed.

Conclusion: RAPA influences liver regeneration by attenuating genes important for hepatocyte proliferation, angiogenesis and hepatic stellate cell activation in the early but not in the late phase of liver regeneration. This down-regulation may represent a new therapeutic target for a safe use of RAPA in reduced-size liver grafts.

\section{O096}

QUALITY OF CARE FROM THE PERSPECTIVE OF BREAST CANCER PATIENTS AFTER SURGERY: THE QUOTE BREAST CANCER INSTRUMENT

M. de Kok ${ }^{1}$, H.J. Sixma ${ }^{2}$, K.J. Spijkers ${ }^{2}$, T. van der Weijden ${ }^{3}$, C.J.H. van de Velde $^{4}$, J.A. Roukema ${ }^{5}$, F.W. van der $\mathrm{Ent}^{6}$, A.V.R.J. Bell M.F. Meyenfeldt ${ }^{1}$

1 University Hospital Maastricht, Maastricht, The Netherlands

2 NIVEL, Utrecht, The Netherlands

3 Maastricht University, Maastricht, The Netherlands

4 Leiden University Medical Center, Leiden, The Netherlands

5 St. Elisabeth Hospital, Tilburg, The Netherlands

6 Orbis Medical Center, Sittard, The Netherlands

7 Laurentius Hospital, Roermond, The Netherlands

Objectives of the study were to develop a questionnaire that would:

1) produce specific data on health care services related to breast cancer;

2) address issues that are related to the needs and expectations of individual patients;

3) represent values that had been formulated in collaboration with patients;

4) measure quality of health care services from the perspective of patients who had been operated on for breast cancer.

Patients who had been operated on for breast cancer participated in 8 focus groups (FG) (72 participants) and 6 concept mapping (CM) sessions (65 participants) in five hospitals in the Netherlands. During CM, the most important items from the focus groups were structured according to similarity and rated on a 5-point Likert type scale ranging from 1 (relatively unimportant) to 5 (extremely important). A pilot questionnaire was developed based on their results and sent to 614 patients who had experienced breast-cancer surgery. Aspects were formulated as 'performance' and 'importance' statements with 4-point Likert type response scales. Item, inter-item and factor analysis were performed to evaluate the internal consistency of the subscales, the presumed factor struc- 
ture and the feasibility of the instrument. Reliability was represented by Cronbach's Focus groups resulted in 221 aspects on quality of care mentioned by the respondents. Importance scores of the 81 aspects included in the $\mathrm{CM}$ ranged from 1.4 to 4.7 . The 3 most important items that addressed clinicians were "caregivers should have all results available when I have an appointment with them for that reason', 'a check-up 6 months after surgery should be part of the protocol' and 'the results of the biopsy are told to me within a week after the test'. Structuring according to similarity resulted in 6 general clusters of items: period of admission ( 6 items), focus on patient (9 items), respect for the patient (12 items), time schedule (10 items), continuity of care (10 items) and education (8 items). The remaining 26 items were labelled as miscellaneous. The results of the pilot questionnaire showed that all 10 items with the highest performance scores concerned the breast care nurse's and surgeon's approach. Six of the 10 items with the lowest scores addressed waiting times. Knowledge transfer covered 6 of the 10 items with the highest scores on importance level. Least importance was attached to familiarity with the surgeon. The final version of the questionnaire contains 32 items on quality of care. Factor analysis confirmed an underlying taxonomy of subscales of items. Reliability for the subscales (Cronbach's a) varied between 0.67 and 0.93 .

Focus groups and concept mapping resulted in items this specific patients group considers as reflecting quality of care. The pilot questionnaire proved useful in which items to include in the final questionnaire. The study resulted in a self-administered questionnaire that is easy to complete, anonymous, contains proper scientific characteristics and determines patients perceptions on quality of breast cancer care. The questionnaire provides specific information on where room exists for improvement in breast cancer care in different care settings.

\section{O097}

\section{BARRIERS AND FACILITATORS FOR IMPLEMENTATION OF AN ULTRA SHORT STAY PROGRAMME FOR BREAST CANCER IN 4 EARLY ADOPTER HOSPITALS: THE MADO STUDY}

M. de Kok ${ }^{1}$, T. van der Weijden ${ }^{1}$, A. Kessels ${ }^{1}$, C.D. Dirksen ${ }^{1}$, C.J.H. van de Velde $^{3}$, J.A. Roukema ${ }^{4}$, F.W. van der Ent ${ }^{5}$, A.V.R.J. Bell ${ }^{6}$,

M.F. Meyenfeldt ${ }^{1}$

1 University Hospital Maastricht, Maastricht, The Netherlands

3 University Medical Center Leiden, Leiden, The Netherlands

4 St. Elisabeth Hospital Tilburg, Tilburg, The Netherlands

5 Orbis Medical Center, Sittard, The Netherlands

6 Laurentius Hospital Roermond, Roermond, The Netherlands

The MaDO study ('Breast cancer surgery in ultra short stay') is aimed at identifying barriers and facilitators for implementation of breast cancer surgery in ultra short stay and formulating key recommendations that care professionals can take into account when preparing for change from clinical to ultra short stay admission. Based on the results of an earlier study in the University Hospital Maastricht (uhM), the study protocol was developed. In 2004 the uhM started the prospective MaDO study which is carried out in four other hospitals in the Netherlands. For analysis of the barriers and facilitators, data were collected derived from interviews, monthly meetings, e-mails and telephone conferences. Those were analysed, potential barriers and facilitators for successful implementation were extracted and statements were formulated, categorised according to themes. More than 40 barriers and facilitators were identified. The corresponding themes were: guideline, caregiver, patient, colleagues, finances and organisation. Most barriers concerned organisational (e.g. limited opening hours of the short stay ward and ICT problems) and guideline-related aspects (e.g. limited flexibility of surgical planning or insufficiënt analgetic and anti-emetic drug policy). A patient related- barrier concerned the income-dependant fee that patients must pay for home care nursing. An example of a financial barrier was the lower reimbursement that was applied by insurance companies with the change from a clinical to an ultra short stay setting for breast cancer surgery. Organisational-type facilitators (e.g. admission on the day of surgery) were most common. An example of a caregiver-related facilitator is the wish to participate in (inter)national trends (e.g. to develop a transmural care team). A set of relevant indicators for implementation of the ultra short stay programme for breast cancer surgery was described. Several of our key recommendations for successful implementation of this programme are:

1) availability of a flexible planning for all breast-related surgeries;

2) following a day care admission protocol tailor-made to minimise pain and nausea postoperatively;
3) financial incentive by the insurance company which stimulates the change to day care admission

4) financial organisational structure which prevents the loss of income for surgeons when shifting their practice to day care surgery.

These indicators can be used by any professional preparing to start with breast cancer surgery in ultra short stay.

\section{O098}

THE VIRULENCE OF STAPHYLOCOCCCUS AUREUS AND STAPHYLOCOCCUS EPIDERMIDIS STRAINS ISOLATED FROM CLINICAL SOURCES

A. Dominiak' ${ }^{1}$ D. Zdzalik' ${ }^{1}$ H. Galkowska ${ }^{1}$, B. Interewicz ${ }^{1}$ W.L. Olszewski ${ }^{1}$, E. Stelmach ${ }^{1}$, M. Luczak ${ }^{2}$, Z. Machowski ${ }^{3}$ 1 Medical Research Center, Warsaw, Poland 2 Department of Microbiology, Medical School, Warsaw, Poland 3 Department of Surgery, Limpopo University, Polokwane, South Africa,

Staphylococcus aureus can be frequently isolated from surgical site infections whereas S.epidermidis colonizes intravenous devices and may even cause septicemia. The knowledge of the phenotype of isolates and their antibiograms, although clinically useful, does not give insight into the virulence of the isolated microbes.

Aim: of the study was to characterize the virulence of S.aureus and S.epidermidis isolates obtained from skin surface, wounds and deep tissues of hospitalized patients and skin surface of non-hospitalized volunteers. Pathogenicity of bacterial species is determined by their ability to produce virulence factors involved in colonization and invasion of host tissues (i.e. adhesins, invasins, extracellular enzymes and toxins). We selected $S$.aureus genes encoding bacterial surface proteins binding fibronectin (fnb-A) and collagen (cna) and gene for exfoliatin A (eta)and S.epidermidis genes atIE encoding autolysin and icaAB for polysaccharide intercellular adhesin (PIA). Resistance to methicillin of Staphylococci was also estimated.

Methods: S.aureus $(n=42)$ and S.epidermidis $(n=22)$ isolates were obtained. Strains were identified by using Chapman base, Slidex Staphkit and API STAPH test after overnight at $37^{\circ} \mathrm{C}$ bacteria culture in BHI base. Genomic DNA was isolated using Roche High Pure Template Kit. Presence of genes was examined in PCR reaction with specific primers. PCR products were detected on $2 \%$ agarose gel electrophoresis and staining with ethidium bromide. The distribution of gene mecA conferring the methicillin resistance was compared with the result of susceptibility tested by ATB STAPH method.

Results: Genes encoding adhesins and the exfoliatin A were found mostly in S.aureus isolates originating from wounds and deep tissues (fnbA - $50 \%$, cna- $72,2 \%$, eta- $27,8 \%$ ) and from skin surface of hospitalized patients (fnbA- $15,4 \%$, cna- $53,8 \%$, eta- $23,1 \%$ ) compared to nonhospitalized volunteers (fnbA- $0 \%$, cna- $9,1 \%$, eta- $9,1 \%$ ). S.epidermidis gene altE was present in all examined strains whereas gene icaAB in $87.5 \%$ strains harvested from skin surface of hospitalized and nonhospitalized subjects and in $71,4 \%$ of strains isolated from wounds and deep tissues. Comparison of results obtained by PCR and conventional method for staphylococcal resistance to methicillin estimation showed discrepancies in case of S.aureus isolates, suggesting the need for using of both methods in clinically difficult cases of infection.

Conclusion: Detection of genes encoding adhesions fnb-A and cna as well as gene for epidermolitic toxin eta can be useful for evaluation of virulence of $\mathrm{S}$.aureus isolates.

\section{O099}

\section{CORRELATING GENERIC AND DISEASE SPECIFIC QUALITY OF} LIFE MEASURES WITH THE CLINICAL INDICATORS OF LOWER LIMB ISCHEMIA

S. Gulati', H.L.D. Lee ${ }^{2}$, M.N. Abdul Rahman², A. Mekako², P. Coughlin ${ }^{3}$, P.T. McCollum², I.C. Chetter ${ }^{2}$

1 Hull Royal Infirmary, Hull, United Kingdom

2 Hull Royal Infirmary, Hull, United Kingdom

Introduction: This study analyses the impact of lower limb ischemia (LLI) on quality of life (QoL) assessing the correlation between generic and disease specific QoL measures with clinical indicators of LLI.

Methods: We prospectively studied a consecutive series of 555 patients (344 men; median age 69 [IQR: 63-75] years), presenting with varying degrees of LLI prior to intervention. Clinical indicators of LLI included: patient reported walking distance (PRWD), initial claudication (ICD) and 
maximal (MWD) treadmill walking distances and ankle-brachial pressure indices (ABPIs) at rest ( $r$ ) and post exercise (pe). QoL analysis was performed with the Short Form 36 (SF36 - generic) and Kings' College VascuQol (disease specific) questionnaires respectively.

Results: Increasing LLI results in a statistically significant deterioration, in all 8 domains of SF36, SF36 Index and disease-specific quality of life $(p=0.000$, Kruskal-Wallis ANOVA).

$\begin{array}{llllll}\text { Score } & \text { PRWD } & \text { ICD } & \text { MWD } & \text { ABPIr } & \text { ABPIpe } \\ \text { Physical Function } & 0.579^{*} & 0.509^{*} & 0.551^{*} & 0.205^{*} & 0.161^{*} \\ \text { Physical Role } & 0.361^{*} & 0.263^{*} & 0.306^{*} & 0.221^{*} & 0.187^{*} \\ \text { Bodily Pain } & 0.462^{*} & 0.440^{*} & 0.428^{*} & 0.139^{*} & 0.121^{*} \\ \text { General Health } & 0.282^{*} & 0.231^{*} & 0.286^{*} & 0.039 & 0.010^{*} \\ \text { Vitality } & 0.379^{*} & 0.422^{*} & 0.438^{*} & 0.121^{*} & 0.078 \\ \text { Mental Health } & 0.269^{*} & 0.268^{*} & 0.361^{*} & 0.061 & 0.008 \\ \text { Social Function } & 0.425^{*} & 0.388^{*} & 0.441^{*} & 0.153^{*} & 0.043 \\ \text { Emotional Role } & 0.388^{*} & 0.310^{*} & 0.361^{*} & 0.225^{*} & 0.128^{*} \\ \text { SF36 Index } & 0.510^{*} & 0.458^{*} & 0.508^{*} & 0.171^{*} & 0.101^{*} \\ \text { VascuQoL } & 0.662^{*} & 0.545^{*} & 0.558^{*} & 0.308^{*} & 0.252^{*}\end{array}$

${ }^{*} \mathrm{p}<0.05$ (values shown are spearman rank correlation coefficients)

Conclusion: Patients with mild claudication experience better QoL than those with more severe disease. Although statistically significant, there is overall weak correlation between clinical indicators and QoL suggesting other factors apart from walking distance affect QoL Correlation coefficients for disease specific were superior to those for generic QoL instruments, advocating its use in claudication studies

\section{O100}

BILIARY DRAINAGE ATTENUATES POST-ISCHEMIC REPERFUSION INJURY IN THE CHOLESTATIC RAT LIVER

J.J. Kloek, H.A. Marsman, A.K. Van Vliet, D.J. Gouma, T.M. van Gulik Academic Medical Center Amsterdam, Amsterdam, The Netherlands
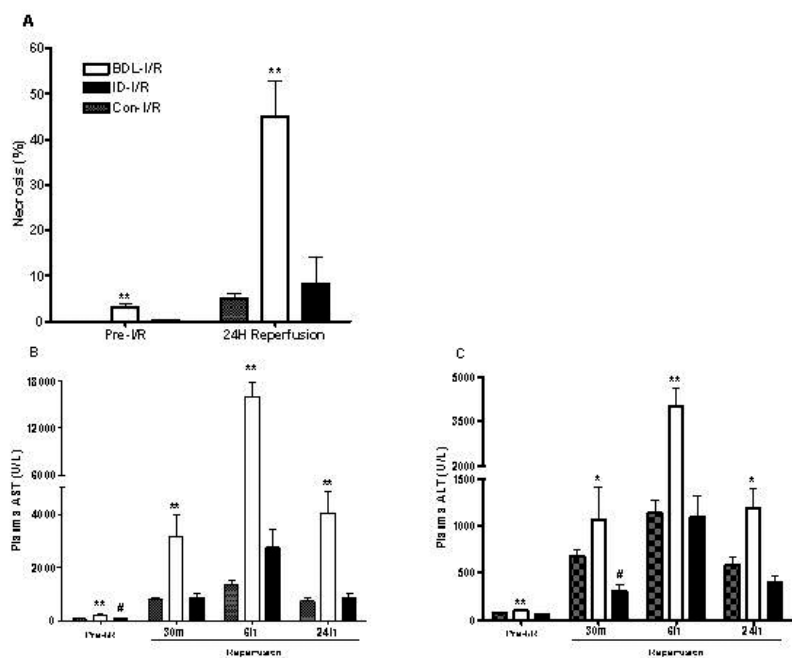

Figure 1: Effect of biliary decompression on liver damage following hepatic lRR injury. Liver injury was measured by (A) histological evaluation ( $\mathrm{H} \& \mathrm{E}$ staining) before ischemia and after 24 hour reperfusio Plasma AST (B) and ALT (C) levels were measured before ischemia, and after 30 minutes, 6 and 24
hours of reperfusion, respectively. At all time points there was a significant increase of damage in the hours of repentusion, respectively. At all time points there was a significant increase of damage in the BDL group as compared to the control and ID group. Values are expressed as mean $\pm S E M$ for
individual animals per group ( $*=p<0.05$ and $\$ p<0.01$ for control and $I D$, $\#=p<0.05$ for control).

Background: Oxidative stress plays an important role in cell death and has been identified as a pathogenic feature of parenchymal injury in cholestasis. During major liver resections, vascular inflow occlusion can be applied to reduce intraoperative blood loss. The combination of ischemia and oxidative stress potentially enhances post-ischemic reperfusion injury and increases the risk of remnant liver failure in cholestatic patients requiring extended liver resection. Biliary decompression relieves oxidative stress in cholestasis. Therefore, preoperative biliary drainage seems a worthwhile intervention in cholestatic patients undergoing major liver resection, although this is debatable in distal tumors which are resected without partial liver resection. The aim of this study was to assess the influence of cholestasis on hepatocellular injury after hepatic ischemia and reperfusion (I/R) injury in a bile duct ligation (BDL) model in the rat. In addition, the effect of biliary decompression prior to I/R was studied.

Methods: Male Wistar rats were randomized into three groups: The first group $(n=6)$ underwent partial liver ischemia after 7 days BDL. Partial liver ischemia was induced by clamping of the segmental portal triad to the median and left lateral lobes $( \pm 70 \%)$ for 30 minutes. The second group $(n=6)$ underwent internal drainage (ID) after 7 days BDL and following 5 days, was subjected to ischemia. Control animals $(n=6)$ underwent two sham laparotomies at 7 and 12 days, respectively, and subsequently ischemia. A reperfusion period of $24 \mathrm{~h}$ was applied in all groups after which the rats were sacrificed. To assess the effects of $\mathrm{BDL}$, ID and sham operations, rats ( $\mathrm{n}=6$ per group) were sacrificed before inducing liver ischemia. Blood was collected before ischemia, and after $30 \mathrm{~min}, 6 \mathrm{~h}$ and $24 \mathrm{~h}$ of reperfusion, respectively. The following outcome parameters were assessed: plasma AST and ALT, hepatic oedema, lipid peroxidation, histopathology, hepatic synthetic function (albumin and prothrombin time), inflammatory response \{plasma IL-6, IL-10, IFN-y and GRO/KC (a chemoattractive cytokine in rodents), hepatic myeloperoxidase (MPO) activity\} and the total antioxidant activity (a marker of antioxidant status) of the liver.

Results: Peak levels of AST and ALT were significantly higher at $6 \mathrm{~h}$ reperfusion as compared to the control and ID group (BDL group: AST:15977 $\pm 4595 \mathrm{U} / \mathrm{L}$; ALT:3987 $\pm 1560 \mathrm{U} / \mathrm{L}$ control group: AST:1328 $\pm 429 U / L ; A L T: 1140 \pm 329 U / L$ and ID group: AST:2750 $\pm 1508 U / L$; ALT:1097 $\pm 497 \mathrm{U} / \mathrm{L}(\mathrm{p}<0.01)$. Plasma IL-6, IL-10 and GRO/KC were all significantly increased in the cholestatic group over 24 hour reperfusion $(p<0.05$ for control and ID). Prothrombin time, MPO, necrosis, lipid peroxidation and oedema were all significantly increased in the BDL group after $24 \mathrm{~h}$ reperfusion ( $\mathrm{p}<0.05$ for control and ID). Moreover, the antioxidant activity was strongly decreased in the cholestatic group $(p<0.01$ for control and ID). No significant differences for most parameters were seen in the ID group as compared to the control group.

Conclusion: The cholestatic rat liver is more susceptible to post-ischemic reperfusion injury, probably mediated by decreased antioxidant activity and an increased inflammatory response. These injurious effects were attenuated by biliary decompression. The results of this study suggest an increased risk of inflammatory events, such as clamping of the portal triad during liver resection, in the presence of cholestasis.

\section{O101}

\section{EFFECTS OF ENTERAL -GLUCAN IN A RAT MODEL OF SHORT} BOWEL ON INTESTINAL ADAPTATION AND ANASTOMOTIC HEALING

M. Sener, A.E. Canda, M.A. Kocdor, H. Kocdor, O. Sagol, R. Cehreli Dokuz Eylul University, Izmir, Turkey

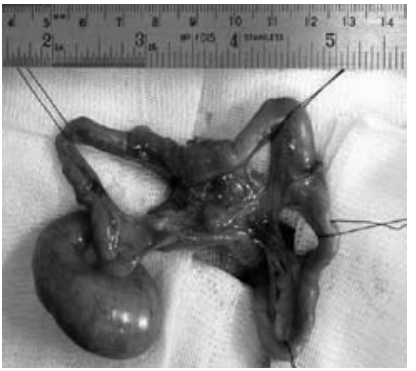

Intestinal length in $\beta$-glucan group-sacrification

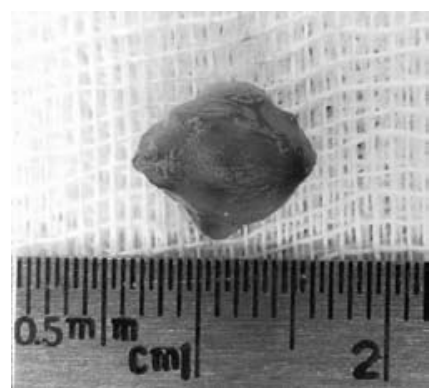

Mesenteric lymph node in $\beta$-glucan group

Background \& aims: Short Bowel Syndrome is a clinical phenomenon which occurs after significant length of small intestinal resection, and mainly characterized by malnutrition, diarrhea, steatore, vitamin deficiency, and electrolyte imbalance and weight loss. After massive small intestinal resection gross and microscopically changes begins immediately in the remnant small intestine called intestinal adaptation. Although many experimental and clinical studies have been performed for enhancing intestinal adaptation, results are controversial. $\beta$-glucan is an immunopharmacological agent which consist of polysaccharide fiber. It has various effects including wound and intestinal anastomotic healing. 
The aim of the study was to determine the effects of orogastric ${ }^{2}$ glucan administration on intestinal adaptation and anastomotic healing in a rat model of SBS.

Methods: Thirty wistar rat were included in this study. Rats were divided in to 3 groups (sham, control, and treatment). In control and treatment group $80 \%$ small bowel resection was performed. In treatment group, ${ }^{2}$ glucan $25 \mathrm{mg} /$ day given by orogastric feeding route. Rats were sacrified on 14th day. Weights, intestinal lengths and diameters, anastomotic burst pressures and blood lymphocyte counts were recorded.

Results: In treatment group intestinal length and blood lymphocyte count were increased, statistically significantly; there were no statistically difference between groups in rat weights and anastomotic burst pressure values

Conclusion: In this study we observed the effects of ${ }^{2}$-glucan on intestinal length after massive intestinal resection, as well as the effects of ${ }^{2}$ glucan on intestinal lenfoproliferation.

\section{2}

OROGASTRIC BENEFIBER ENHANCES INTESTINAL ADAPTATION IN A RAT MODEL OF SHORT BOWEL SYNDROME

T. Ekice ${ }^{1}$, A.E. Canda ${ }^{2}$, H. Kocdor ${ }^{1}$, M. Fuzun ${ }^{1}$, O. Dokanakoglu' ${ }^{1}$, O. Sagol ${ }^{1}$, M.A. Kocdor ${ }^{1}$

1 Dokuz Eylul University, Izmir, Turkey

2 Manisa State Hospital, MANISA, Turkey

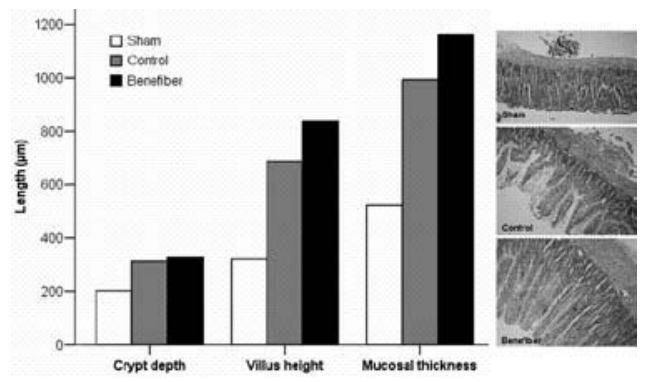

Histopathological findings

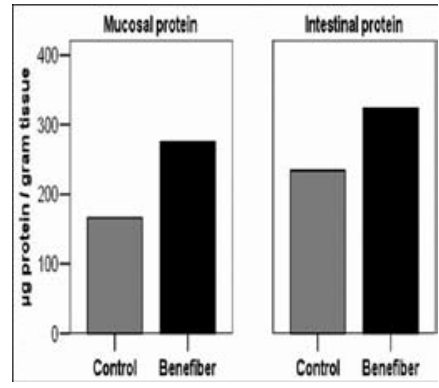

Assesment of mucosal and intestinal protein

Background \& aims: Short Bowel Syndrome is a clinical condition which occurs after significant length of small intestinal resection, and mainly characterized by malnutrition, diarrhea, nutrient deficiency, and weight loss. After massive small intestinal resection gross and microscopically changes begins immediately in the remnant small intestine called intestinal adaptation. Although many experimental and clinical studies have been performed for enhancing intestinal adaptation, results are controversial. Benefiber $®$ is a partially hydrolyzed guar gum compound which consists of polysaccharide fiber. High dietary fiber stimulates intestinal mucosal proliferation by activating peptide hormones. The aim of the study was to determine the effects of orogastric Benefiber supplementation on intestinal adaptation in a rat model of SBS.

Methods:Twenty-seven male Wistar rats $(213-300 \mathrm{~g})$ were randomly divided into 3 groups: Sham group rats $(n=7)$ underwent bowel transection and reanastomosis; Control group rats $(n=10)$ underwent $80 \%$ small bowel resection; Benefiber group rats $(n=10)$ underwent $80 \%$ small bowel resection and were treated with orogastric Benefiber $(0.125 \mathrm{~g} /$ day $)$ for 13 days. The intestinal adaptation was quantified with morphological (mucosal thickness, villous height and crypt depth) and biochemical analysis (mucosal and intestinal protein levels), and blood samples were collected for serum albumin and total protein levels.

Results:Throughout the experiment, there was no significant dif- ference in body weight changes between the Control group and the Benefiber group. Benefiber treatment significantly increased intestinal villous height and mucosal thickness $(P=0.014$ and $P=0.031$, respectively). There was no significant difference between Control group and Benefiber group in terms of crypt depth, goblet cell count and intestinal length. Mucosal protein and intestinal protein levels were also higher in Benefiber group than in Control group $(P=0.002$ and $P=0.019$, respectively). On day 14 , serum albumin and total protein levels were significantly higher in Sham group compared to Control and Benefiber groups $(P<0.000$ and $P=0.041$, respectively); which were similar in Control and Benefiber groups.

Conclusion: These findings suggest a benefit of enteral Benefiber supplementation on intestinal adaptation in a rat model of short bowel syndrome.

\section{O103}

\section{PLASMA DNA: A MOLECULAR MARKER OF SURGICAL INSULT AND POST-OPERATIVE RECOVERY IN ESOPHAGEAL CANCER}

\author{
R.J. Mason', F. Banki', D. Oh¹, J.A. Hagen², S.R. DeMeester ${ }^{1}$, \\ T.R. DeMeester
}

1 University of Southern California, Los Angelos, USA

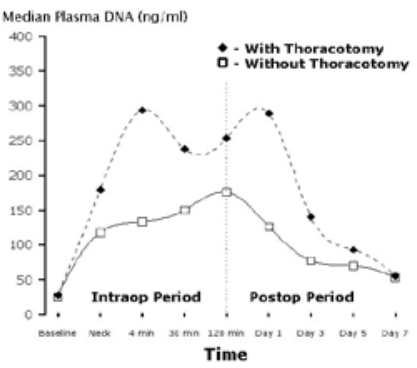

Plasma DNA Intraoperatively and Postoperatively

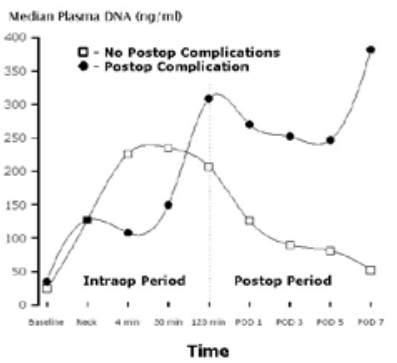

Plasma DNA in Patients with Complications

Background: Plasma DNA is known to be released from cells in response to cellular injury, yet this phenomenon has never been objectively evaluated by using surgical procedures of different magnitudes as a human model or by correlating the plasma DNA to the magnitude of cellular injury during and after these procedures.

Hypothesis: Plasma DNA is a molecular marker that correlates to the degree of cellular injury, and recovery following surgery.

Objective: To assess plasma DNA changes intra-operatively, to relate plasma DNA to the magnitude of the surgical insult and to monitor the changes during the postoperative recovery period.

Material and method: Prospective study of 35 consecutive patients with resectable esophageal cancer who had esophagectomies of different magnitudes: 19 esophagectomy without thoracotomy $\{12$ transhiatal esophagectomy (THE), 7 Laparoscopic thoracoscopic esophagectomy\}, and 16 en bloc transthoracic esophagectomy (en bloc TTE). The plasma DNA was measured prior to surgery, throughout the course of the operation at 4 different intervals, and on postoperative day 1-3-5-7. Mean plasma DNA values were compared.

Results: There was no difference in plasma DNA prior to surgery. A significant difference was seen in the median plasma DNA intraoperatively between the two groups: esophagectomy without thoracotomy (507 $\mathrm{ng} / \mathrm{ml} / \mathrm{min}$ range 211-2708) and en bloc transthoracic esophagectomy (median 1098ng/ml/min range 295-22284), $P=0.0143$, see figure 1 . There was no correlation between the plasma DNA level and tumors size, depth of penetration or number of the lymph nodes resected. 
Post operative complications were identified in 6 patients who demonstrated a significant elevation in plasma DNA on postoperative days 5 and 7 , see figure 2 .

Conclusion: Plasma DNA increases during surgery as a result of cellular damage and the rise correlates with the magnitude of surgery. The descent of plasma DNA postoperatively correlates with surgical recovery. Elevation of the plasma DNA during the postoperative period correlates with postoperative complications. Plasma DNA is an objective molecular marker of surgical insult and can be used to monitor post operative recovery after esophagectomy.

\section{O104}

THE EFFECTS OF ISCHEMIC POSTCONDITIONING ON A RAT MODEL OF HEPATIC ISCHEMIA AND REPERFUSION INJURY

B. Aydogan 1 , A.E. Canda ${ }^{2}$, P. Tuncel ${ }^{1}$, O. Sagol ${ }^{1}$, S. Karademir ${ }^{1}$

1 Dokuz Eylul University, Izmir, Turkey

2 Manisa State Hospital, Manisa, Turkey

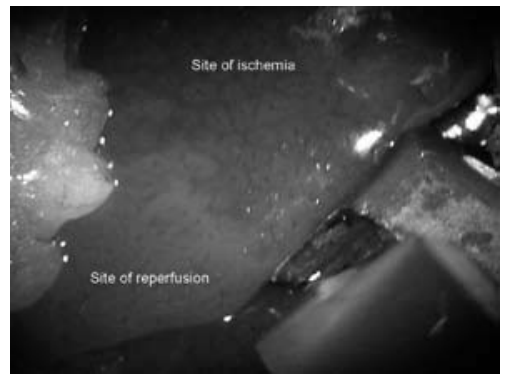

Reperfusion after pedicle declamping

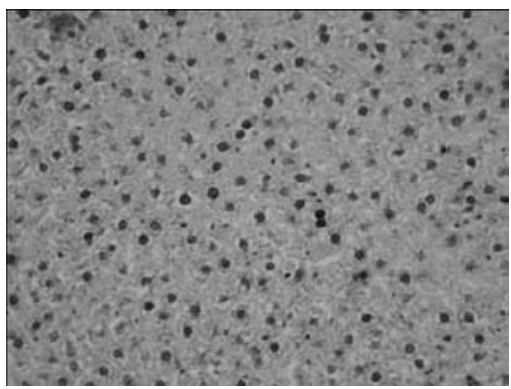

Mitotic activity in hepatocytes, PCNA

Background \& aims: Hepatic pedicle clamping (Pringle maneuver) in major liver surgery results ischemia and reperfusion (I/R) injury. The method of ischemic preconditioning which is composed of short periods of ischemia and reperfusion may be used for several times for preventing I/R injury. Recently, to prevent I/R injury another method, ischemic postconditioning, is proposed which is the application of short periods of I/R during reperfusion after a period of long lasting ischemia. The aim of this study is to evaluate the effects of ischemic preconditioning and postconditioning on preventing the hepatic ischemia and reperfusion injury.

Methods: The study was designed in five groups: Group $1(n=4)$, sham rats; Group $2(n=14)$, hepatic ischemia and reperfusion; Group 3 $(n=14)$, ischemic preconditioning and hepatic ischemia group; Group 4 $(n=14)$, hepatic ischemia and ischemic postconditioning group; Group $5(n=14)$, ischemic preconditioning, hepatic ischemia and ischemic postconditioning group. Ischemia and reperfusion was performed by 60 minutes hepatic ischemia following 120 minutes reperfusion; ischemic preconditioning was performed by 10 minutes ischemia and 10 minutes reperfusion before 60 minutes hepatic ischemia; and ischemic postconditioning was performed by 60 minutes hepatic ischemia followed by 2 minutes ischemia periods and between this periods in increasing duration of reperfusion (2, 3, 5, and 7 minutes) periods. Serum liver enzymes (AST, ALT, LDH) and bilirubin, oxidative stress in the liver (as measured by malondialdehit), histopathological liver parenchymal injury scale and hepatic regeneration (as immunohistochemically determined by mitotic activity in hepatocytes; proliferating cell nuclear antigen; PCNA) were evaluated.

Results: In the early phase after I/R, serum liver enzymes and total bilirubin levels were significantly higher in Group 4 than Group 2 and Group $5(P<0.05)$. Early after I/R, no significant difference was observed in oxidative stress, histopathological parenchymal injury scale and immunohistochemically determined (proliferating cell nuclear antigen; PCNA) mitotic activity between groups; however, on 7 th day after I/R no significant difference was observed between groups.

Conclusion: We observed that ischemic postconditioning do not protect hepatic I/R injury; moreover we observed an increase I/R injury with ischemic postconditioning. The effect of ischemic preconditioning on hepatic I/R injury is superior to the effects of both ischemic preconditioning and postconditioning.

$\mathrm{O} 105$

THE PROLIFERATIVE EFFECTS OF THYROID HORMONE ON RATS WITH SHORT BOWEL SYNDROME

O. Yksel ${ }^{1}$, T. Sahin ${ }^{2}$, A. Poyraz ${ }^{3}$, E. Tatlicioglu ${ }^{2}$, H. Pasaoglu ${ }^{4}$,

E. Ofluoglu 4 , S. Elbeg ${ }^{4}$, B. Salman' ${ }^{2}$, K. Dikmen ${ }^{2}$

1 Gazi University Medical School, Ankara, Turkey

2 Department of Surgery, Ankara, Turkey

3 Department of Pathology, Ankara, Turkey

4 Department of Biochemistry, Ankara, Turkey

Background and aim: Short bowel syndrome (SBS) is a malabsorptive state resulting from a decrease in absorptive surface area following massive small bowel resection. The most important factor contributing to survival following massive bowel resection is the ability of the remaining bowel to adapt. Thyroid hormone is one of the trophic hormones proven to have proliferative effects in the organism. In the present study the effects of thyroid hormone on the adaptation process of the remaining bowel in an experimental model of SBS.

Materials and methods: Rats were randomly assigned to one of the three groups: Group A (Sham rats) underwent bowel transection and anastomosis; Group B (SBS+saline) underwent massive bowel resection and anastomosis, and were administered saline by sc injection on postoperative days 1 and 7 , and group C (SBS+thyroid hormone) underwent massive bowel resection and anastomosis, and were administered triiodothyronine (T3) $400 \mu \mathrm{g} / 100 \mathrm{~g}$ of total body weight by sc route on postoperative days 1 and 7 . All animals were weighed just before and after the surgical procedure and were continued to be weighed every third day. Animals were sacrificed on postoperative 15th day. Following the sacrification $3 \mathrm{ml}$ of blood samples were collected in order to study serum T3 and thyroid stimulating hormone (TSH) levels. In addition the jejunal segment proximal to the anastomosis and ileal segment distal to it were extracted for the assessment of their length, weight, mucosal weight, and DNA/protein ratio and mean diameter. Histopathologically jejunal and ileal villi height, crypt depth, cellular proliferation and apoptosis indices were evaluated.

Results: Weight loss was higher in the group B when compared to group C $(p>0.05)$. Serum levels of T3 were lower in the group B compared group $C(p<0.05)$. The jejunal and ileal weight and length were significantly higher in group $C$ in comparison to group $B(p<0.05)$. The mucosal weight and DNA/protein ratio is significantly higher in group $C$ than other groups $(p<0.05)$. Jejunal diameter was increased in group $C$ when compared to groups A and B $(p<0.05)$. Ileal diameter was found to be increased in group $C$ in comparison to other groups but it was not statistically significant. Jejunal villus height and crypt depth was significantly higher in group $C$ than group $B(p<0.05)$. lleal villus height and crypt depth was found to be higher in group $C$ but no statistical significance was found. Jejunal and ileal cellular proliferative index was found to be statistically higher in group $\mathrm{C}$ when compared the group $\mathrm{B}$; on the other hand only jejunal cellular apoptosis index was significantly lower in group $C(p<0.05)$.

Conclusion: Intestinal adaptation is the progressive recovery from intestinal failure following bowel resection. This process allows the remaining bowel to restore an absorptive surface area and functional capacity for adequate digestion. In the present study, thyroid hormone was found to increase remaining bowel diameter; especially the jejunal segments. It was also found to increase proliferation with the reduction of apoptosis (prominent in the jejunal segments).

\section{O106}

A RISK ASSESSMENT /MANAGEMENT CLINIC REDUCES PREDICTED MORTALITY IN PATIENTS WITH PERIPHERAL VASCULAR DISEASE

S. Gulati ${ }^{1}$, H.L.D. Lee ${ }^{2}$, M.N. Abdul Rahman², A. Mekako², J. Hatfield ${ }^{4}$, P. Coughlin², P.T. McCollum², I.C. Chetter ${ }^{2}$ 
1 Hull Royal Infirmary, Hull, United Kingdom

2 Hull Royal Infirmary, Hull, United Kingdom

Introduction: Nurse-led assessment/management has been described to effective in addressing risk factors in patients with peripheral vascular disease (PVD). We aimed to evaluate whether this transfers into a reduction in predicted mortality due to coronary heart disease.

Method: We prospectively studied a consecutive series of 75 patients (49 men; median age 65 [IQR: 56-74] years), diagnosed with PVD referred to a nurse-led risk assessment/ management clinic, where protocol driven care was utilised to manage, hyperlipidemia, hypertension, antiplatelet medication and lifestyle advice regarding smoking and exercise. was given .Patients also completed Short form36 and Kings' College VascuQol questionnaire All patients were reviewed at a three month interval. Predicted mortality due to coronary heart disease was calculated using the PROCAM and Framingham risk scores.

Results: Visit 1 Visit $2 \mathrm{p}$ value *

PROCAM (\%) 14 (8-30) 10 (4.5-21.0) 0.000

Framingham (\%) 14 (10-25) 11 (7.5-17.0) 0.000

Total Cholesterol (mmol/l) 5.2 (4.5-6.1) 4.5 (4.0-4.9) 0.000

Triglycerides (mmol/l) $1.7(1.2-2.5) 1.4(1.0-2.3) 0.001$

LDL (mmol/l) 3.1 (2.5-3.7) 2.5 (2.1-2.9) 0.000

King's College VascuQoL 4.4 (3.4-5.3) 4.8 (3.8-6.0) 0.030

"Wilcoxon signed rank test

Conclusion: Nurse-led risk assessment/management clinics for patients with PVD is effective in reducing predicted morbidity and mortality due to CHD, as well as improving patients' quality of life.

\section{O107}

PATIENT FACTORS ASSOCIATED WITH WOUND COMPLICATIONS FOLLOWING VARICOSE VEIN SURGERY

A.I. Mekako, P.A. Coughlin, J. Hatfield, S. Gulati, M. Abdulrahman, B. Akomolafe, P. Renwick, B. Johnson, I.C. Chetter, P.T. McCollum Hull Royal Infirmary, Hull, United Kingdom

Introduction: About 50,000 varicose vein operations are performed in the UK NHS annually. Although regarded as clean surgery, wound infection rates of up to $16 \%$ have been reported following varicose vein surgery. Several patient-related factors may be associated with wound complications following surgery. We aimed to determine these factors in varicose vein surgery.

Methods: We studied 427 patients (571 limbs) undergoing groin surgery for greater saphenous varicose veins. Demographic characteristics were obtained at baseline, and patients underwent standard consultant-led surgery. A personal wound assessment logbook was completed over the initial 10-day postoperative period, based on an adapted ASEPSIS scoring method. Continuous and categorical data were analysed by the Mann-Whitney and Chi-Squared tests respectively. Logistic regression analyses were performed to determine relationships between patient variables and wound outcome.

Results: Groups were matched for demographics and risk factors for wound infections. Univariate analysis showed good wound outcomes with being female $(\mathrm{OR}[95 \% \mathrm{Cl}])(1.5[1.2-2.2]) p=0.03$, but poor outcomes with high body mass index $(0.95[0.91-0.99]) p=0.03$ and current smoking $(0.7$ [0.6-0.9]) $p=0.04$. Redo surgery $(0.6[0.4-1.0]) p=0.18$ and high CEAP class $(0.8$ [0.6-1.2]) $p=0.34$ were associated with a poor wound outcome, but this did not reach statistical significance.

Multivariate analysis showed that a higher BMI, being male, and current smoking were independent predictors of a worse outcome.

Conclusion: Male patients, current smokers and those with a high body mass index are at a higher risk for poor wound outcomes and infection following varicose vein surgery. These patients should be considered for prophylactic antibiotics to prevent postoperative wound morbidity.

\section{8}

\section{GENERIC QUALITY OF LIFE ANALYSIS IN CLAUDICANTS: CAN THE SHORT FORM 8 REPLACE THE SHORT FORM 36?}

S. Gulati, H.L.D. Lee, M.N. Abdul Rahman, A. Mekako³ , P. Coughlin, P.T. McCollum, I.C. Chetter

Hull Royal Infirmary, Hull, United Kingdom

Background: Generic Quality of Life (QoL) is a crucial outcome measure in claudicants with the Short Form 36 (SF36) accepted as the 'gold standard' instrument. We aimed to assess whether the new, shorter, simplified Short Form 8 (SF8) is sufficiently responsive to replace SF36 in claudicants.

Patients and methods: 155 claudicants, 113 men and 42 women, median age 66 (range 44-84) years were graded according to ISCVS standards ( 30 mild, 52 moderate and 73 severe claudicants). Patients completed SF36 and SF8 at the time of assessment. Both instruments assess the same $8 \mathrm{QoL}$ domains. Construct and convergent-divergent validity was assessed for both the QoL instruments. Responsiveness of normalised data between grades of claudicants was analysed with nonparametric statistical tests.

Results: Validity.There was greater correlation between like domains of SF36 and the SF8 (0.594-0.792, p=0.000) than the non-like domains, suggesting good convergent'divergent validity. Furthermore, the SF36 and SF8 demonstrated similar construct validity.

Responsiveness. Increase in the severity of the claudication resulted in a statistically significant deterioration in six of the eight domain of both SF8 \& SF36 ( $<<0.05$, Kruskal-Wallis ANOVA). However, comparison of patients with moderate and severe claudication showed no statistically significant difference in 7 of 8 SF36 domains and in any of the SF8 domains ( $p>0.05$, Mann-Whitney U test).

Conclusion: SF8 and SF36 have similar validity and responsiveness in patients with intermittent claudication. In future the simplified SF8 may replace SF36 as the gold standard generic QoL analysis in claudicants.

\section{O109}

\section{DAMPENING THE PERIOPERATIVE INFLAMMATORY RESPONSE WITH HIGH FAT FEEDING IS PREDOMINANTLY CCK-A RECEPTOR DEPENDENT}

T. Lubbers, J.J. de Haan², M. Hadfoune

University Hospital Maastricht, Maastricht, The Netherlands

Patients undergoing major surgery are prone to develop systemic inflammatory response syndrome (SIRS) and sepsis due to an exacerbated inflammatory response. Previously, we discovered that high-fat feeding strongly reduces inflammation and preserves gut barrier function following hemorrhagic shock, a model of systemic inflammation. High-fat feeding induces release of CCK, leading to activation of the autonomous nervous system. Subsequent triggering of efferent vagus fibers results in direct inhibition of the TNF-alpha release from macrophages.

The current study investigates the mechanism via which CCK activates the autonomous nervous system, centrally or peripherally. Male Sprague-Dawley rats were subjected to a model of non-lethal hemorrhagic shock in which $30-40 \%$ of blood volume was withdrawn. Rats were fed with high-fat nutrition, containing high amounts of phospholipids, at 18 hours, 2 hours and 45 minutes before shock. Antagonists against peripherally or centrally located CCK receptors (respectively A or B) were administered 25 minutes before shock in animals fed with high-fat feeding. Plasma and tissue samples were collected at 90 minutes. TNFalpha concentration was measured as a marker of the inflammatory response. Gut barrier function was assessed by measuring translocation of horseradisch peroxidase (HRP) in ileal segments and bacterial translocation to mesenteric lymph nodes, liver and spleen. Blocking the CCKA receptor $(125.2+/-5.9 \mathrm{pg} / \mathrm{ml})$ resulted in significant higher TNF- \pm levels compared to vehicle $(28.0+/-2.0 \mathrm{pg} / \mathrm{ml} ; \mathrm{p}<0.0001)$ and to CCK-B receptor blocking $(96.4+/-8.2 \mathrm{pg} / \mathrm{ml} ; p<0.05)$. Gut permeability was significantly enhanced by blocking the CCK-A receptor $(7.2+/-2.9 \mu \mathrm{g} / \mathrm{ml})$ compared to vehicle $(1.9+/-0.8 \mu \mathrm{g} / \mathrm{ml} ; \mathrm{p}<0.0001)$ and compared to CCK-B receptor blocking $(5.1+/-2.1 \mu \mathrm{g} / \mathrm{ml} ; \mathrm{p}<0.01)$. Blocking the CCKA receptor $(88.7+/-7.5 \mathrm{CFU})$ resulted in significantly worsened bacterial translocation compared to vehicle $(33.2+/-3.0 \mathrm{CFU} ; \mathrm{p}<0.0001)$ and compared to blocking the CCK-B receptor $(55.8+/-3.7$ CFU; $p<0.001)$. In conclusion, these data suggest that the anti-inflammatory actions of high-fat feeding are predominantly mediated via the peripheral CCK-A receptors. However, a minor role for the centrally located CCK-B receptors was observed. Defining the mechanism by which high-fat feeding, through release of CCK can exert its anti-inflammatory effect contributes to the clinical use of high-fat feeding in reducing exacerbated inflammatory responses related to major surgery.

0110

ASSESSMENT OF PRE- AND POSTOPERATIVE LIVER REGENERATION AND TUMOR GROWTH IN PATIENTS UNDERGOING PREOPERATIVE PORTAL VEIN EMBOLIZATION

W. de Graaf, K. P. van Lienden, A.K. van Vliet, R.J. Bennink, 
T. M. van Gulik

Academic Medical Center Amsterdam, Amsterdam, The Netherlands

Preoperative portal vein embolization (PVE) is an option in patients requiring major liver resection in whom estimated future remnant liver (FRL) is considered insufficient. PVE induces atrophy of the embolized, tumor-bearing lobe while compensatory hypertrophy occurs of the non-embolized lobe, resulting in increased FRL volume and function. There is evidence that tumor growth after PVE is accelerated, creating a dilemma in terms of optimal waiting time between PVE and resection. It is also uncertain whether postoperative liver regeneration is hampered after preoperative PVE. Time interval between PVE and resection is mainly determined by the time required to reach sufficient FRL hypertrophy, as usually assessed by CT volumetry. Safe resection can be performed if $\mathrm{FRL}$ exceeds $25-30 \%$ of total liver volume in patients with normal livers, or $40 \%$ in patients with compromised livers. Few methods however, are available to measure FRL function after PVE. 99mTc- mebrofenin hepatobiliary scintigraphy (HBS) enables the measurement of both total and regional liver function. The aims of this study were to assess tumor growth as well as increase in FRL volume and function after PVE, and to compare postoperative liver regeneration in patients undergoing preoperative PVE with normal postoperative liver regeneration using CT volumetry and HBS. From 2005 until 2006, PVE was performed in thirteen patients. Preceding PVE, total liver volume, tumor volume and FRL volume was measured by CT volumetry. Total and FRL function were determined by HBS. Three to four weeks after PVE, CT volumetry was performed to measure the increase of FRL volume and tumor growth in the embolized lobes. HBS was used to measure the increase of liver function. Three months after liver resection, HBS and $C T$ volumetry were repeated to assess postoperative liver regeneration. A group of 7 patients who underwent a right (extended) hemihepatectomy without PVE in the same period was used as postoperative control group. Of eight patients with measurable tumors in the liver, seven patients showed increased tumor volume after PVE (23.5\% increase). One patient with a benign lesion had decreased tumor volume. Increase of FRL function ( $41.8 \%$ increase) measured by HBS was significantly $(p<0,05)$ greater than increase of FRL volume (32.6\% increase), 3-4 weeks after PVE. Correlation between functional increase and volumetric increase of the FRL was moderate $(r=0.63, p 0.03)$. In 9 patients, a partial hepatectomy was performed. In the PVE group, liver volume regenerated up to $78 \pm 18 \%$ of initial liver volume whereas liver function increased to $85.5 \pm 21 \%$ of preoperative values. In the control group, liver volume and function recovered to $74.0 \pm 9.8 \%$ en $85.4 \pm 12.6 \%$ of initial values, respectively. In this study, tumor load increased after PVE. Therefore, the time interval between PVE and resection is crucial and should be limited. Increase of FRL function is more advanced than increase of volume. Functional assessment of the FRL after PVE using HBS might be more reliable than CT-volumetry to determine the time period between PVE and liver resection. Preoperative PVE has no negative effect on postoperative liver regeneration.

\section{O112}

RADIOFREQUENCY ABLATION: VALUABLE ADDITION OF A LOCAL TREATMENT METHOD IN THE MANAGEMENT OF PATIENTS WITH LIVER METASTASES

M.R van den Bergh, M.R. Meijerink, C. Sietses, M.P. van den Tol, M.A. Cuesta, S. Meijer

University Medical Center Amsterdam, Amsterdam, The Netherlands

Resection is considered the gold standard for management of patients with hepatic malignan-cies. However, despite evolving indications of resectability, still $70-80 \%$ of patients are un-suitable candidates for hepatic resection. Radiofrequency ablation (RFA) is a liver-directed treatment strategy with promising results in achieving local tumor control. The aim of this retrospective single-centre study is to describe the results of RFA alone or in combination with resection in patients with secondary hepatic malignancies. All patients were followed to assess complications, treatment response and recurrence. From June 2001 to February 200640 patients (24 male, 16 female) with a median age of 66.5 years (22-82) were treated 44 times with RFA. The primary tumor was colorectal carcinoma in 34 patients, gonadal malignancies in 4 patients (ovarian $(n=2)$, testicular $(n=2)$ ) and renal cell carcinoma in 1 patient. One patient had hepatic metastases from an unknown primary. In total, 85 lesions with a mean diameter of $2.3 \mathrm{~cm}(0.2-8.0)$ were ablated with a median number of 2 metastases (1-7) per patient. In 9 patients RFA was combined with resection and 14 patients received chemotherapy before the RFAprocedure. Four patients that were treated with RFA had intrahepatic recurrent disease following previous resection. There were no procedure-related deaths. Minor complications occurred in 5 patients. Two patients had a pleural effusion, necessitating a chest tube in one patient. One patient developed a pneumonia and another patient was diagnosed cholangitis. One patient had an unexplained neuropathy of the right femoral nerve, unrelated to the RFA-procedure. After a median follow-up of 18.5 months (3-56) 21 (53\%) patients had recurrent disease. There was one local recurrence at the RFA site, 13 elsewhere intrahepatic and 6 extrahepatic metastases. One patient developed new liver as well as lung metastases. Six (15\%) patients had died, 3 of metastatic disease. This study suggests that if hepatic metastases are unresectable, RFA can be of great value to achieve a considerable prolongation of survival. Unfortunately, recurrent disease is a common phenomenon. Proper patient selection and multidisciplinary management of patients with co-lorectal liver metastases are equally crucial. An aggressive treatment strategy can provide prolongation of survival and even curation in selected cases, emphasizing the necessary close collaboration between surgical and medical oncologists.

\section{3}

\section{ULTRASOUND-GUIDED RADIOFREQUENCY ABLATION OF EARLY} BREAST CANCER IN A RESECTION SPECIMEN: FIRST RESULTS OF A FEASIBILITY STUDY

D.L. Kreb, M.J.C.M. Rutten, J.C. van der Linden, J.F.M. Pruijt, K. Bosscha Jeroen Bosch Medical Center, 's-Hertogenbosch, The Netherlands

Introduction: Over the past two decades there has been a transition towards less invasive local treatment of breast cancer, without altering survival rates. Nowadays minimally invasive techniques, such as radiofrequency ablation (RFA) are being studied as local treatment of invasive breast carcinoma. We performed ex vivo ultrasound-guided RFA of breast carcinomas to determine the feasibility of this promising technique and evaluated the histological findings.

Materials and methods: Radiofrequency ablation was performed of invasive ductal carcinoma ' diagnosed by core needle biopsy ' in postmenopausal women immediately after the surgical procedure (lumpectomy or mastectomy). A needle was placed in the centre of the tumour using ultrasound guidance. Subsequently, the tumour was ablated for a period of 12 minutes. Pathologic evaluation of the specimen was performed using conventional HE staining as well as cytokeratin 8 staining and $\mathrm{NADH}$ diaphorase to assess cell viability.

Results: Up to now, 13 patients with an average age of 66,9 years (range $56,5-77,8$ ) were included in this study. The mean tumour size was $13 \mathrm{~mm}$ (range 7-23). All procedures were technically successful. Pathology revealed complete cell death in 11 lesions, but viable cells were found in two lesions: in one lesion cytokeratin 8 staining revealed a microscopic focus of viable tissue at the ablation zone margin and viable cells were found along the needle tract in another lesion. In one patient the target lesion was completely destroyed, but viable ductal carcinoma in situ (DCIS) was found outside the ablated area.

Conclusion: Ultrasound-guided radiofrequency ablation of invasive breast cancer can result in complete cell death, but a high level of accuracy is required in positioning the needle electrode correctly in the tumour. These results support the use of RFA in the treatment of localized breast carcinoma, and an in vivo study has been initiated in our institution to determine the feasibility, safety and complications of this procedure.

\section{O114}

\section{CRYOABLATION INDUCES GREATER INFLAMMATORY AND} COAGULATIVE RESPONSES THAN RADIOFREQUENCY ABLATION (RFA) OR LASER INDUCED THERMOTHERAPY (LITT) IN A RAT LIVER MODEL

M.C. Jansen 1 , R. van Hillegersberg ${ }^{1}$, M. Levi², I. Schoots ${ }^{2}$,

H. Crezee ${ }^{2}$, J. Beek ${ }^{2}$, T.M. van Gulik ${ }^{2}$

1 Utrecht Medical Center, Amsterdam, The Netherlands

2 Academic Medical Center, Amsterdam, The Netherlands

Background \& aim: Local ablative techniques such as Cryoablation (CA), Radiofrequency ablation (RFA) and Laser-induced thermotherapy (LITT) are alternative therapies for patients with unresectable liver tumours. Major complications of cryoablation are renal and pulmonary failure ('cryoshock phenomenon') most likely caused by an exaggerated 
inflammatory and coagulative response. We investigated whether there are different inflammatory and coagulative responses between various local ablative techniques.

Methods: Livers of 48 rats were subjected to either CA, RFA, LITT or sham operation ( $n=12$ in each group). Blood was withdrawn before, and 1, 3, 6 and 24 hours after ablation of an equal amount of liver. Liver enzymes, as well as inflammatory and coagulation parameters were determined. Whole liver sections from the coagulated liver lobe were stained for quantification of necrosis and morphological examination. Results: Histological examination showed similar volume of complete destruction of liver parenchyma after CA, RFA, or LITT. Transaminase levels following CA were significantly higher than following RFA or LITT, and remained high at 24 hours. The inflammatory response upon CA, as reflected by white blood cell count and cytokine levels, was significantly higher than following RFA or LITT. The systemic intravascular procoagulative state in rats that underwent $\mathrm{CA}$, as reflected by platelets, and levels of sensitive markers for activation of coagulation and fibrinolyis, was significantly higher than following RFA or LITT.

Conclusion: $\mathrm{CA}$ of liver in rats induces greater inflammatory and coagulative responses than RFA or LITT. The combined activation of inflammation and coagulation may importantly contribute to the higher morbidity after $\mathrm{CA}$.

\section{5}

THE IMPACT OF INTRAOPERATIVE FINDINGS ON THE SURGICAL TREATMENT STRATEGY OF PATIENTS WITH COLORECTAL LIVER METASTASES

M.R. van den Bergh, M.R. Meijerink, C. Sietses, M.P. van den Tol, M.A. Cuesta, S. Meijer

University Medical Center Amsterdam, Amsterdam, The Netherlands

Imaging techniques play a crucial role in the assessment of patients with colorectal liver metastases (CRLM). Intraoperative ultrasonography (IOUS) is considered the gold standard to accurately identify (size/number) and localize hepatic metastatic lesions. Computed tomography (CT) scans are used preoperatively to map the CRLM and determine resectability. However, the accuracy of modern helical CT scanning is largely undetermined. Also, it is uncertain to what extent discrepancies between preoperative imaging and intraoperative findings (IOUS + surgical exploration) affect surgical decision-making and treatment strategy. The aim of this single-centre study is to evaluate the accuracy of preoperative CT scanning compared to intraoperative findings in patients treated for CRLM and whether discrepancies changed treatment strategy. All patients who underwent a laparotomy because of CRLM between January 2000 and March 2006 were included. All patients had preoperative helical CT scanning followed by IUOS of the liver. Also, surgical exploration was conducted to assess evidence of local disease and/or peritoneal metastases. IOUS plus surgical exploration was compared to $\mathrm{CT}$ and surgical reports were analyzed to detect changes in treatment strategy. In this 6 -year period, 100 patients with CRLM had a laparotomy 117 times with the intent of surgical treatment (resection and/or radiofrequency ablation). IOUS alone had different findings in $26 \%$ compared to the preoperative CT scan; more lesions in $16 \%$, additional information on localization in $9 \%$. IOUS and surgical exploration combined changed the surgical treatment strategy in $37 \%$. In only 3 patients, new information did not alter the surgical treatment. Preoperative CT failed to predict peritoneal metastases in 4 patients (carcinomatosis in 2 patients) and diffuse hepatic metastases in 3 patients. Also, in 7 patients surgical exploration found evidence of local extension of the CRLM not seen on CT. However, IOUS plus surgical exploration deemed patients inoperable in only 5 cases. IOUS \pm surgical exploration provided new or additional information regarding number, localization and/or local extension of the CRLM in $39 \%$ of cases and subsequently almost invariably changed the surgical treatment strategy. The combination of IOUS and surgical exploration is an essential diagnostic tool in the assessment of patients with CRLM undergoing surgical treatment.

0116

QUANTITATIVE GENOMIC EXPRESSION OF OESTROGEN-RECEP. TOR RELATED GENES IN EARLY BREAST CANCER AND THEIR RELATIONSHIP TO OUTCOME

J.T. Garvin, R.E. McNeill, E. Hennessy, N. Miller, M.J. Kerin National University of Ireland, Galway, Galway, Ireland

Introduction: We identified four genes commonly associated with breast cancer outcome in gene expression studies; GATA3, a transcription activator, BCL2-asssociated athanogene 1 (BAG1), a multi-functional protein which interacts with steroid hormone receptors, matrix metalloproteinase 9 (MMP9) and MMP11. This study quantitatively examines expression of these genes in axillary-node-negative (ANN) breast cancer and correlates gene expression with survival.

Methods: We selected 14 axillary-node-negative breast cancer patients who had recurrence within five years and matched these with 14 patients whose disease had not recurred on long-term follow-up. Quantitative real-time PCR was performed on RNA extracted from fresh-frozen tumour tissue with gene specific primers using HPRT and GUS as controls and pooled normal breast tissue as a baseline for gene expression. Results: GATA3 was over-expressed in good prognosis patients relative to poor prognosis $(p<0.001)$. In poor-prognosis patients GATA3 was over-expressed in ER-positive relative to ER-negative patients $(p<0.01)$. In ER-negative disease good prognosis patients over-expressed GATA3 relative to poor prognosis $(p<0.01)$. Increased expression of BAG1 was associated with better five-year survival and improved response to treatment, particularly in ER-positive patients $(p<0.01)$. MMP9 was increased in poor prognosis patients relative to good prognosis. MMP9 and MMP11 were over-expressed in ER-positive tumours relative to ERnegative $(p<0.01)$. In poor-prognosis patients MMP11 was overexpressed in ER-positive versus ER-negative groups $(p<0.01)$.

Conclusion: Increased expression of GATA3 and BAG1 are predictive of improved survival in ANN breast cancer. Increased expression of MMP9 and MMP11 are associated with poor outcome in ANN breast cancer. All four genes are overexpressed in ER-positive disease. These genes may have an important future role in the prognostic profiling of breast cancer patients.

\section{O117}

SURGICAL ASPECTS OF EDEMA OF LOWER LIMBS LYMPHOSCINTIGRAPHIC EVALUATION

\author{
M. Cakala1 ${ }^{1}$ J. Cwikla², W.L. Olszewski \\ 1 Medical Research Center, Warsaw, Poland \\ 2 Nuclear Medicine Laboratory, Warsaw, Poland
}

Clinically edema of the limb looks alike irrespective of its etiology The knowledge of the causes and tissue changes in the extremity is necessary for establishing diagnosis. Recent progress in lymphoscintigraphy, with use of tracer bound to aggregated or non-aggregated albumin injected intradermally or intravenously, helps to differente various types of edema of limbs. Identification of etiological factors of edema is necessary for rational therapy, as physiotherapy, elastic support, antibiotics or microsurgery.

Material \& methods: Lymphoscintigraphy of lower limbs was performed in groups: 1. Acute and chronic circumscribed dermatitis, 2. Posttraumatic edema, 3. Postsurgical edema after cancer operations (hysterectomy). 4. Postthrombotic syndrome. 5. Edema of unknown etiology with asymptomatic onset. Technique of lymphoscintigraphy. The $99 \mathrm{mTc}$ Nannocol $(3 \mathrm{mCi})$ was injected intradermally into the first toeweb. Scans were performed 30, 90 and 150 minutes after injection. The patients were allowed to walk freely the same distance between the two scanning procedures. The lymphoscintigrams were analyzed densitometrically using the Olympus Microimage version 3 . Three areas were evaluated: 1.inguinal (lymph nodes), 2.thigh (lymphatics) and3. calf (lymphatics). The sum of surface area of lymphatics and nodes was calculated. The values were expressed as an index of edema/control limbs.

Results: There were 72 males and 114 females. The left limb was affected in $52 \%$ and right in $37 \%$ and both in $8 \%$ Duration of edema was $133 \pm 123$ months. In group 1 , the inguinal area of edematous limb ratio to normal limb was 0.235 , in the thigh 1.58 , and calf 2.41 . Uneven outline of lymph nodes, dilatation of thigh and calf lymphatics was observed. Group 2 was characterized by small nodes and dilated lymphatics. The node ratio values were 0.569 , of thigh 1.42 and calf 2.2 . In group 3 , the nodes were unchanged with ratio values 0.94 , dilated thigh 2.32 and calf 2.67. Group 4 was characterized by enlarged nodes with ratio 1.18 , thigh 2.84 and calf 1.9. Different values were obtained in group 5. There were small nodes with normal outline and few limb lymphatics. The node ratio values were 0.4 , of thigh 1.98 and calf 2.95 . In each group major changes in the lymphatic system were observed. Generally, with longer duration of edema more advances destructive changes were seen in inguinal nodes and subsequently dilatation of peripheral lymphatics. Color Doppler investigation showed partial thromboses in group 4 and $30 \%$ of cases in the posttraumatic group 2. Conclusions. Edema of 
lower limbs requires lymphoscintigraphic imaging to evaluate the degree of damage of the lymphatic system and differentiate lymphedema from thrombophlebitis, posttraumatic and the so called idiopathic edema. Different treatment is advocated for different types of edema.

\section{8}

\section{NEUROPROTECTIVE FEATURE OF GLUTAMATE RECEPTOR AN-} TAGONISM AFTER ACUTE COLON OBSTRUCTION

\section{J. Kaszaki ${ }^{1}$, D. Erces ${ }^{2}$, A. Racz ${ }^{2}$, Z. Palasthy' ${ }^{2}$, L. Vecsei ${ }^{2}$, M. Boros ${ }^{2}$} 1 University of Szeged, Szeged, Hungary

Background: Obstruction of the gastrointestinal tract is a frequent emergency surgical situation, with high morbidity and mortality rates. Intestinal obstruction and inflammatory bowel diseases are characterized by an increased frequency of bowel movements. In this respect, various data suggest that the local production of glutamate receptor agonist metabolites may be involved in the pathogenesis of motility disorders. Glutamate is a major excitatory neurotransmitter in the CNS, and thus it is likely to play a role as an excitatory neurotransmitter in the enteric nervous system too. Our aims were to characterize the changes in motility patterns and inflammatory reactions following treatment with Compound FR, a nonspecific antagonist of all subtypes of ionotropic glutamate receptors, under normal circumstances and during acute colon obstruction in a large animal model.

Methods: Occlusion of the mid-transverse colon was maintained for $420 \mathrm{~min}$ in anesthetized dogs. The systemic hemodynamics [cardiac output and total peripheral resistance (TPR)], Imesenteric circulatory changes (blood flow of superior mesenteric artery; SMA) and plasma levels of nitrite/nitrate (NOx) were monitored. Strain-gauge transducers were used to analyze motility changes on the hepatic and lienal flexures, respectively. Groups 1 and 2 served as sham-operated controls, while in groups 3 and 4 transverse colon obstruction was induced by tourniquet. Groups 2 and 4 were treated with Compound FR $(50 \mathrm{mg} / \mathrm{kg}$ i.v.) at $180 \mathrm{~min}$. Tissue biopsies were taken from the oral part of the colon to determine the activities of xanthine oxidoreductase (XOR) and myeloperoxidase (MPO; a marker of leukocyte accumulation) activities. Results: The obstruction induced a hyperdynamic circulatory reaction, which was accompanied by significant increases in the plasma NOx level, colon XOR activity and leukocyte accumulation, and a steady rise in the motility index. The Compound FR treatment prevented the obstruction-caused decrease in TPR and increased the tone of the colonic smooth muscles, but permanently decreased the motility index of the characteristic, giant contractions of the colon. The administration of Compound FR significantly inhibited the obstruction-induced increases in colon XOR activity and leukocyte accumulation also.

Conclusion: Our results indicate the decisive modulatory role of the glutamate receptors in early colonic motility alterations after occlusion. Compound FR treatment has additional cytoprotective effects related to an indirect inhibition of XOR-derived superoxide radical production and leukocyte activation. Supported by research grant RET-08/04

\section{9}

\section{IMMUNOGLOBULIN M, C-REACTIVE PROTEIN AND COMPLEMENT} ACTIVATION: TIME COURSE IN RAT HEPATIC ISCHEMIA-REPERFUSION INJURY

G.M.P. Diepenhorst ${ }^{1}$, W. de Graaf ${ }^{2}$, H.W. Niessen ${ }^{3}$, A.K. van Vliet ${ }^{1}$, T.M. van Gulik ${ }^{2}$

1 Academic Medical Center Amsterdam, Amsterdam, Netherlands

2 Academic Medical Center, Amsterdam, The Netherlands

3 University Medical Center, Amsterdam, The Netherlands

Introduction: A role for C-reactive protein (CRP) and Immunoglobulin $M(\operatorname{IgM})$ in the initiation of $\mathrm{I} / \mathrm{R}$ injury via complement activation has been established in various I/R models. Both CRP and IgM can bind to neoantigens in the damaged cell membranes leading to the activation of complement. However, the time course in which IgM and CRP activate complement in liver $\mathrm{I} / \mathrm{R}$ remains unknown.

Aim: The aim of this study was to assess the relationship in time of $\lg M$ and CRP binding in comparison to complement activation in a hepatic I/R rat model.

Methods: Male Wistar rats $(n=60)$ were divided into eleven groups: 60 minutes of partial ischemia $(70 \%)$ followed by $0,3,6,12$ or 24 hours of reperfusion (each $n=6$ ). Partial ischemia was induced via clamping of the left segmental portal triad. Sham laparotomy groups with corres- ponding reperfusion times were included, as well as a control group sacrificed before ischemia (each $n=5)$. Hepatocellular injury (plasma aminotransferases and histopathology), inflammatory response (neutrophil influx), prothrombin time (PT), rat IgM content in ischemic hepatic tissue homogenates and immunohistochemical depositions of CRP, IgM and $\mathrm{C} 3$ on frozen tissue sections were assessed during each reperfusion time.

Results: Aspartate aminotransferase (AST) and alanine aminotransferase (ALT) significantly increased after 3 hours and remained elevated up to 24 hours, peaking at 6 hours $(5300 \pm 1000$ and $3100 \pm 800 \mathrm{U} / \mathrm{L}$, respectively) ( $p<0.05$ versus sham). Hepatic neutrophil influx was significantly increased from 3 to 6 hours of reperfusion $(p<0.05)$, peaking at 12 hours $(1.1 \pm 0.2 \mathrm{U} / \mathrm{mg}$ protein) $(p<0.05)$. An increase in prothrombin time (PT) was observed throughout reperfusion ( $p<0.05$ versus sham). Histopathological injury increased from 3 to 12 hours of reperfusion and remained elevated thereafter ( $p<0.05$ versus sham). Rat IgM content in ischemic tissue homogenates demonstrated a progressive increase throughout reperfusion up to 24 hours ( $p<0.05$ versus sham). Immunohistochemical analysis showed a similar staining pattern of IgM and C3 (Spearman rank-correlation test $r(S)=0.79, p<0.01$ ), in which depositions increased from 3 to 6 hours of reperfusion and significantly decreased at 24 hours. CRP depositions peaked at 3 hours of reperfusion and significantly decreased throughout reperfusion until 24 hours ( $p<0.05$ versus 3 hours).

Conclusion: These data suggest that both IgM and CRP are mediators of hepatic I/R-induced complement activation in rats, in which CRP-mediated activation appears to precede that of IgM.

\section{O120}

\section{HEPATIC EXPRESSION AND REGULATION OF METALLOTHIONEIN AND HEME OXYGENASE GENES IN ENDOTOXEMIC RATS WITH} OBSTRUCTIVE JAUNDICE THE ROLE OF KUPFFER CELLS

\section{S. Abraham ${ }^{1}$, Ferencz², A. Szabo², G. Lazar², M. Boros², E. Hermesz²,} G. Lazar $\mathrm{Jr}^{2}$

1 University of Szeged, Szeged, Hungary

Introduction: The liver is highly sensitive to endotoxin (ETX)-triggered systemic inflammation and the cellular stress response includes an enhanced Kupffer cell activity with increased production of proinflammatory cytokines and reactive oxygen species. Liver expression of antioxidant heme oxygenize (HO) and metallothionein (MT) genes are rapidly upregulated by these mediators and/or ETX itself, and this reaction may be protective against cellular stress and inflammation. Our previous study has shown that Kupffer cell blockade with gadolinium chloride ( $\mathrm{GdCl} 3)$ has beneficial consequences in endotoxemia following experimental bile duct ligation (Lazar G Jr et al. Inflamm Res 2002). The aim of this study was to characterize a possible link between GdCl3-induced Kupffer cell depletion and the expression of $\mathrm{HO}$ and MT genes in endotoxemic rats with obstructive jaundice.

Methods: For the detection and semiquantitative determination of MT and $\mathrm{HO}$ transcripts, oligonucleotides specific for MT-1/MT-2 and HO-1/ $\mathrm{HO}-2$ isoforms were selected and used in reverse transcription coupled polymerase chain reactions (RT-PCR). Total RNAs for template of RT reactions were isolated from the livers of the following groups: control animals with sham operation $(n=5)$; in group $2(n=5)$ extrahepatic bile duct ligation (BDL) was induced 3 days prior to measurements; in group $3(n=5)$ endotoxemia was elicited by injecting a low dose of E.coli lipopolysaccharide iv. $2 \mathrm{hr}$ before the examinations (ETX group); in group 4 $(n=5)$ the previous challenges were combined $(B D L+E T X)$. In the next series, the same experiments were repeated in four groups of rats $(n=5$, each), but these animals were pretreated with $\mathrm{GdCl} 3(1 \mathrm{mg} / 100 \mathrm{~g} \mathrm{bw})$ iv $24 \mathrm{hr}$ before the experiments.

Results: The expression of MT-1 and MT-2 genes was altered in a stress- and isoform-specific manner: BDL alone resulted in a significant decrease in both MT-1 and MT-2 mRNA levels. ETX and GdCl3 treatments by themselves led to a significant increase in the MT-2 expression (approx. 3.5 fold), while the MT-1 mRNA level was unchanged. The isoform-specific stimulatory effect of $\mathrm{GdCl} 3$ on MT expression was also observed in $\mathrm{BDL}+\mathrm{GdCl} 3$ and $\mathrm{BDL}+\mathrm{ETX}+\mathrm{GdCl} 3$ treatments; though the MT-2 mRNA levels were lower than after $\mathrm{GdCl} 3$ or ETX treatments alone. Activation of $\mathrm{HO}$ genes by $\mathrm{BDL}$ and $\mathrm{ETX}$ was also isoform specific; BDL and ETX treatment caused an approx. 3.5- and 10-fold increase in the HO-1 mRNA level, respectively, while HO-2 expression was only moderate (approx. 2.5-fold). $\mathrm{GdCl} 3$ decreased the ETX- and BDL-mediated $\mathrm{HO}-1$ mRNA accumulation by approx. $50 \%$. As a consequence 
of $\mathrm{GdCl} 3$ pretreatment, ETX-activated $\mathrm{HO}-2$ gene expression was also repressed.

Conclusion: Experimental obstructive jaundice aggravated by endotoxemia significantly induced the expression of MT-2 and HO-1 genes. Kupffer cell blockade influences an antioxidant defense system, already activated by endotoxemia and/or cholestasis. The alteration in the expression of the MT and $\mathrm{HO}$ genes is isoform specific, which indicates a specific role for these genes in these conditions.

\section{1}

\section{STENT OR SURGERY IN PALLIATION OF BILIARY OBSTRUCTION} DUE TO IRRESECTABLE PANCREATIC CANCER. CLINICAL OUTCOMES AND COST-EFFECTIVENESS ANALYSIS

\author{
M.A. Elsebae, A.S. Sadek \\ Theodor Bilharz Medical Research Institute, Caïro, Egypt
}

\section{Biliary-enteric surgical bypass has been the treatment of choice} for palliation of malignant obstructive jaundice for many years. The introduction of a minimally invasive endoscopic stenting technique has provided a new option for clinicians, and this has become a very popular procedure. The choice between surgery and endoscopic drainage still a matter of personal preference and availability of alternative techniques. However, in developing countries, cost-effectiveness has to be considered. The aim of this study was to compare the clinical outcomes, complications, and costs between endoscopic plastic stent insertion and surgery drainage in patients with irresectable cancer of the head of the pancreas. A special emphasis on technical considerations at surgery is done. Seventy-seven of patients with a clinical diagnosis of advanced cancer of the head of the pancreas were palliatively treated at our institute at the last four years. Open surgical biliary bypass was performed for thirty-two and endoscopic drainage was performed for forty-five patients. Outcome measures were as follows: effectiveness of biliary drainage, hospital stay, complications either early or late; mortality and hospital costs. Surgery was found to have nearly the same effectiveness of endoscopic biliary drainage, a longer hospital stay and but lower overall expense than the stenting. The early complications rate was $15.6 \%$ following surgical biliary-enteric bypass and $11.1 \%$ following endoscopic stent drainage. The follow-up period ranged from two to 13 months (mean 8.5 months). The surgical approach resulted in less recurrent jaundice than the stent approach. Recurrent jaundice from stent blockage was the most common late complication in patients treated by the endoscopic method ( 15 blockages in 11 patients), with a median patency time of 3 months (range, 1 ' 9 months). There were only three late complications in the surgical group, which was a significantly lower rate than in the stent group $(p=0.007)$. The number of re-admissions was higher in patients treated by endoscopic biliary drainage than that following surgery. $33.3 \%$ patients who initially had a gallbladder bypass required a subsequent biliary endoscopic intervention at early or late postoperative period, but none of the patients who initially had a bile duct bypass. It was concluded that surgery is recommend for palliation of biliary obstruction due to irresectable pancreatic cancer in the patients at low-risk in a setting such as our country, where reasonable cost-effectiveness must be considered. Compared to patients whose initial biliary bypass was to the bile duct, the risk of having recurrent jaundice is substantially greater in patients whose initial bypass was to the gallbladder. Key Words: obstructive jaundice, stent, surgery.

\section{2}

CULLIN -1 AND -2 PROTEINS EXPRESSION IN COLON CANCER: CORRELATIONS WITH CLINICOPATHOLOGIAL VARIABLES

\author{
O.M. Michail ${ }^{1}$, S. Theocharis ${ }^{2}$, K. Giaginis ${ }^{3}$, K.G. Kouraklis ${ }^{4}$, \\ J. Griniatsos ${ }^{5}$, P. Michail ${ }^{5}$ \\ 1 University of Athens, Laiko Hospital, Athens, Greece \\ 2 Department of Forensic Medicine - Toxicology, Athens, Greece \\ 3 Department of Forensic Medicine - Toxicology, Athens, Greece \\ $42^{\text {nd }}$ Dept Propaedeutic Surgery, Athens, Greece \\ $51^{\text {st }}$ Dept of Surgery, Laiko Hospital, Athens, Greece
}

Background \& aims: The cullin family of proteins is involved in the ubiquitin/mediated degradation of cell cycle regulators. The aim of this study was to examine immunohistochemically the expression of Cullin (CUL)-1 and -2 proteins in colon cancer tissue specimens in correlation with clinicopathological variables.

Materials and methods: CUL-1 and -2 protein expression was exa- mined immunohistochemically on paraffin embedded tissue sections from 96 colon cancer cases. CUL-1 and -2 protein positivity, extent of expression (on a four step scale) and intensity (mild, moderate, intense) of staining were correlated with patients'age, gender and TNM stage, tumor histological grade, proliferative capacity (Ki-67 labeling index) and p53 protein expression.

Results: CUL- 1 and -2 protein positivity was noted in $86 \%$ and $98 \%$ of the examined cases, respectively. CUL-1 positivity was statistically significantly correlated with that of CUL-2 $(p=0.029)$ and $p 53(p=0.012)$. The extent of CUL-1 and -2 expression presented a trend with tumor histological grade ( $p=0.072$ and $p=0.056$ respectively).

The intensity of CUL-1 and -2 immunostaining was not correlated with any of the examined variables.

Conclusion: CUL-1 and CUL-2 proteins were widely expressed in colon cancer cases examined and were correlated with important clinicopathological variables. Further molecular and clinical studies are needed in order to delineate the clinical importance of these proteins in the management of colon cancer patients.

\section{O123}

\section{LIVERRESECTION FOR COLORECTAL LIVERMETASTASES: IS A MORE AGGRESSIVE APPROACH JUSTIFIED?}

J.G. Bloemen, P.N.J. Bollars, M.M.E Coolsen, R.M. van Dam, S.W.M.

Olde Damink, M.H.A. Bemelmans, C.H.C. Dejong

University Hospital Maastricht, Maastricht, The Netherlands

Introduction: In recent years the management options for patients with multiple colorectal cancer liver metastases (CRCLM) have vastly improved. Resection of multiple bilobar metastases in multi-step procedures, circumstantially preceded by portal vein embolization, inductive chemotherapy or combined with ablative techniques, are only limited by the residual volume of the liver remnant. For patients with irresectable disease new chemotherapeutic regimens create a potentially curative situation by downsizing liver metastases to resectable disease in $13 \%$ of patients.

The aim of the study was to assess the influence of intensified patient recruitment and a more aggressive treatment strategy, on mortality, morbidity and survival and on numbers of resected livers, after liver resection for CRCLM at our hospital.

Methods: Literature was reviewed for contemporary treatment strategies in colorectal cancer liver metastases. A consecutive series of patients undergoing liver resection for CRCLM after January 1st 2000 (group I), when intensified patient recruitment and more aggressive treatment strategies for patients with CRCLM were introduced at our hospital, was studied. Data on number of liver resections, mortality, morbidity and survival were collected prospectively and then compared with historical data derived from a retrospectively collected database of all patients identified in the hospital information system from 1990-1999 (group II).

Results: The mean follow up of the whole studygroup was 6,4 years (range $0,1-13,7$ ). The follow-up for group I was 1,6 year (range $0,1-5,4$ years) and for group II 4,6 years (range $0,1-13,7)$. The annual median number of liverresections increased from 4 (1-7) in group II to 18 (7-28) in group I. The postoperative morbidity decreased from $40,6 \%$ in group II to $30,8 \%$ in group I (NS). The mortality declined from $6,3 \%$ in group II to $1,0 \%$ in group I $(p=0.07)$. The overall 3 -year survival for group I was $71 \%$ compared to $53 \%$ for group II (NS). The overall 5 year survival was $44 \%$.

Conclusion: In specialized centres resectional liver surgery is safe with a mortality of less than $2 \%$ and an acceptable morbidity. In spite off a 5 year survival of over $40 \%$ and a long term survival of up to $27 \%$, a significant amount of patients with resectable colorectal cancer liver metastases are probably not being referred or not given the chance to downsize liver metastases to resectable disease by combination chemotherapy. Improving awareness of the curative potential and safeness of modern liver surgery, regional multidisciplinary collaboration and more aggressive treatment strategies can increase the number of patients undergoing liver resection for colorectal cancer liver metastases without decreasing long-term outcome.

\section{O124}

\section{DYNAMIC GRACILOPLASTY FOR FECAL INCONTINENCE}

J. Melenhorst ${ }^{1}$, B. Wunnik' ${ }^{1}$ S. Koch ${ }^{2}$, O. Uludag ${ }^{3}$, M.J. Rongen ${ }^{4}$,

B. Geerdes ${ }^{1}$, J. Konsten ${ }^{2}$, W. van Gemert ${ }^{1}$, C. Baeten ${ }^{1}$ 
1 Academic Hospital Maastricht, Maastricht, The Netherlands 2 Viecuri Hospital Venlo, Venlo, The Netherlands 3 Atrium Medical Center Heerlen, Heerlen, The Netherlands 4 Maasland Hospital Sittard, Sittard, The Netherlands

Introduction: Fecal incontinence is a socially disabling condition with a major influence on patient's quality of life. Dynamic graciloplasty (DGP) is indicated after failure of conservative treatment. The aim of this procedure is to create a new functioning anal sphincter by wrapping the gracilis muscle around the anal canal and stimulate muscle contraction with intramuscular electrodes connected to an implantable pulse generator Methods: Patients were treated with dynamic graciloplasty from 1986 until 2006. All data were prospectively collected. The follow-up (FU) periods, after the initial training session of 8 weeks, were at 26 and 52 weeks and annually hereafter. We used the Williams incontinence score to evaluate the success. Anal manometry was performed to objectivate the function.

Results: A total of 319 patients entered the study. The mean FU was 6,2 years $(s d+/-3,7)$. The median preoperative Williams score was 5 . This was significantly reduced during the last FU to $3(p<0.0001)$. A mean voltage of $2.1 \mathrm{~V}(\mathrm{sd}+/-1.2)$ resulted in a mean squeeze pressure of $101.1 \mathrm{~mm} \mathrm{Hg}$ (sd +/- 39.2) during the last FU. This was significantly $(p<0.0001)$ higher than mean baseline squeeze pressure $(82.9 \mathrm{~mm} \mathrm{Hg}$ +/- sd 25.9). 243 complications were registered. Constipation occurring most frequent $(n=51)$. To date 40 patients have been converted to a stoma and 46 patients use retrograde colonic irrigation for constipation or persisting incontinence.

Conclusion: Dynamic graciloplasty is an effective procedure to improve continence in the majority of patients with end-stage fecal incontinence. Although morbidity is high, most problems can be resolved and do not lead to explantation

\section{O125}

\section{PREOPERATIVE CHEMORADIATION IN LOCALLY ADVANCED REC} TUM CARCINOMA USING ORAL CAPECITABINE

A.F.J. de Bruin, J.J.M.E. Nuyttens, A.S.T. Planting, C. Verhoef, J.H.W. de Wilt

Erasmus Medical Center Rotterdam, Rotterdam, The Netherlands

Preoperative radiation therapy in combination with 5-fluoracil (5-FU) improves local tumour control in locally advanced rectal cancer. The aim of our study was to evaluate the toxicity and efficacy of preoperative chemoradiation using oral 5 -FU (Capecitabine $R$ ) in locally advanced rectal cancer. Between July 2005 and august 2006, sixty patients with locally advanced rectal cancer (cT3/T4 and/or N+) were treated with preoperative chemoradiation. Radiotherapy consisted of a total dose of $50 \mathrm{~Gy}$ delivered in 25 fractions of $2 \mathrm{~Gy}$ to the pelvis. Chemotherapy was administered concurrent with radiotherapy and consisted of oral capecitabine $(1650 \mathrm{mg} / \mathrm{m} 2 /$ day in two doses) only on radiotherapy days (5 days a week). Surgery was performed 6 to 10 weeks after completion of chemoradiation. The patient population consisted of 19 female and 41 male with an median age of 61 years (range 32 ' 82 ). All but two patients received the full dose of chemoradiation. A 56 year old male had severe chest pain while taking capecitabine. The second patient a 49 year old female experienced grade 3 diarrhea which required intravenous fluid replacement. Two (3\%) patients developed Grade 3 radiation dermatitis and one grade 3 diarrhea. No Grade 3 or 4 hematologic toxicities developed. All patients received definitive surgery, which consisted of 19 abdominal perineal resections, 25 low anterior resections and 16 Hartmann resections. One patient with a low anterior resection developed an anastomotic leakage (4\%). Final pathology demonstrated; T0 $=8(13 \%)$, $\mathrm{T} 1=6(10 \%), \mathrm{T} 2=14(23 \%), \mathrm{T} 3=27(45 \%), \mathrm{T} 4=5(8 \%)$. Primary tumour and nodes downstaging occurred in $67 \%$ and $84 \%$ of the patients, respectively. Two (3\%) patient had an R1-resection; one after an abdominal perineal resection and one after an low anterior resection. Conclusion: Preoperative chemoradiation with oral capecitabine appears to be safe and well tolerated. In addition, this preoperative treatment has a considerable down staging effect on the tumour and lymph nodes.

\section{6}

ANATOMICAL STUDY OF THE AORTA WITH REGARD TO SEX, HEIGHT AND AGE IN THE GREEK POPULATION

D.A. Lappas, I. Gisakis, A. Smyrnis, E. Kyriopoulou, E. Sarantopoulos, A. Giannopoulos, P. Scandalakis

University of Athens, School of Medicine, Athens, Greece
Objective: The aorta is the arterial stem with the largest width and all arteries of the human body originate from it (systemic circulation). The objective of this study was the measurement of the aorta's dimensions (length, diameter) from its origin to its division to the common iliac arteries. The present announcement constitutes part of a broader study that correlates with the vessels of the neck, the thoracic cavity and the abdomen.

Materials \& methods: The study was performed on 220 corpses ( 115 men and 105 women) between the age of 20-75 years (mean age $=55.83$ years) at the Forensic Agency of Athens during the forensic examination. The study does not include corpses of individuals that suffered from aortic aneurysms at any point of the aorta. The research conditions allowed us to perform wide anatomic incisions. The diameter of the aorta was measured at: 1 . the origin of the aorta, 2. the middle of the ascending aorta, 3 . the middle of the aortic arch, 4 . after the origin of the left subclavian artery, 5 . the middle of the thoracic aorta, 6 . after the aortic foramen of the diaphragm, 7 . the middle of the distance between the origin of the celiac artery and the superior mesenteric artery, 8. after the origin of the renal arteries, 9 . the division of the abdominal aorta to the common iliac arteries.

Results: Generally, it was observed that the diameter of the aorta varies: a) $2.3-3.1 \mathrm{~cm}$ at the origin of the aorta, b) $1.8-2.5 \mathrm{~cm}$ at the end of the aortic arch, c) $1.3-2.6 \mathrm{~cm}$ at the level of the thoracic aorta, and d) $0.7-1.5 \mathrm{~cm}$ at the level of the abdominal aorta $(p>0.05)$. There is statistical significant difference $(p<0.05)$ in the width of the aorta: the width of the aorta is smaller in short individuals (height $<1.65 \mathrm{~cm}$ ) in relation to tall individuals (height $>1,80 \mathrm{~cm}$ ). The differences between the two sexes were not statistically significant $(p=0,643)$. Variations were also observed concerning the structure of the aorta, which are analyzed and will be presented during the congress.

Conclusion: The results of this study showed important variations of the diameter and structure of the aorta. Their knowledge is more than essential for surgeons during surgical procedures, as well as examinations with imaging techniques.

0127

IGF-1-COATED SUTURES IMPROVE ANASTOMOTIC HEALING AFTER DISTAL COLON RESECTION IN EXPERIMENTAL INFLAMMATORY BOWEL DISEASE

E.J.M.Rijcken, T. Fuchs, L. Sachs, S.T. Mees, M. Bruewer,

H.U. Spiegel, N. Senninger, C.F. Krieglstein

University of Muenster, Muenster, Germany

Background: Anastomotic leakage after bowel resection is a severe complication in visceral surgery. Patients with inflammatory bowel disease (IBD) have an increased risk for postoperative bowel leakage. The aim of the present study was to improve anastomotic healing by local application of Insulin-Like-Growth Factor (rhIFG)-1 by coated suture material in a rodent model of IBD.

Methods: Acute colitis was induced in female Sprague-Dawley rats (BW $200 \mathrm{~g}$ ) by oral application of dextran sodium sulfat (DSS) $5 \%$ (wt/v). Inflammatory activity was assessed by clinical disease activity index (DAI), histological score, and myeloperoxidase (MPO)-levels. 7-0 PDS sutures were coated with rhIGF-1 (5\% wt/v) dissolved in poly(D,L)-lactide) (PDLLA, MW 30kD) in a cold fashion. A $2 \mathrm{~cm}$ distal colon segment was resected on the 7th day of inflammation in isoflurane/N2O-narcosis. Primary end-to-end anastomosis was performed in a seromuscular running fashion using microsurgical technique. On 1, 3, and 7 days after surgery bursting pressure was measured in vivo $(n=10)$ and compared with healthy, DSS, and DSS + PDLLA control groups. Statistics: SSPS Results: In the IGF-1 group DAI, MPO, and histological scores were significantly increased on the day of surgery compared to the healthy control group (DAI $9.2 \pm 0.4$ vs. $0.0 \pm 0.1$ pts, MPO $150.8 \pm 21.1$ vs. $54.6 \pm 11.9 \mathrm{U} / \mathrm{g}$, histology $20 \pm 3.1$ vs. $2.2 \pm 0.7 \mathrm{pts})$. There were no significant differences in inflammatory activity within all groups receiving DSS. The percentage of bowel segments ruptured in the anastomotic area was significantly decreased in the IGF-1 group 1 and 3 days after surgery compared to the group receiving DSS alone (20 and $30 \%$ vs. 50 and $66 \%, P<0.05$ ). On the first postoperative day the bursting pressure was significantly higher in the IGF-1 group compared to the DSS group $(261 \pm 4$ vs. $176 \pm 24 \mathrm{mmHg}, \mathrm{P}<0.05)$, but not anymore on the third postoperative day. PDLLA had no effect on anastomotic healing There were no significant differences in bursting pressures 7 days after surgery among any groups. Anastomotic healing in the DSS group was not significantly impaired compared to the healthy controls (bursting 
pressure day 1 after surgery $176 \pm 7$ vs. $192 \pm 5 \mathrm{mmHg}, \mathrm{P}=\mathrm{n}$.s.) Discussion: In the early postoperative phase IGF-1 coated sutures significantly improve anastomotic healing in the distal colon. By coating the suture material high concentrations of IGF-1 can be applied locally, potentially limiting systemic side effects. Thus IGF-1 coated suture materials might beneficial for patients who are at special risk for anastomotic leakage. However, in our model of colitis anastomotic healing was not impaired.

\section{O129}

FIRST RESULTS OF A PRE-OPERATIVE CHECKLIST TO PREVENT PROBLEMS WITH LAPAROSCOPIC TECHNICAL EQUIPMENT IN THE OPERATION ROOM

E.G.G. Verdaasdonk', L.P.S. Stassen², W.F. Hoffmann²,

M. van der Elst ${ }^{2}$, J. Dankelman ${ }^{1}$

1 Delft University of Technology, Delft, The Netherlands

2 Reinier de Graaf Groep Delft, Delft, The Netherlands

Introduction: The introduction of sophisticated technical equipment in minimally invasive surgery has created new problems in the domain of man-machine interaction during operations. A previous study revealed a high incidence of problems with technical equipment. The introduction of a structured pre-operative checklist could prevent these problems. The effect of such a list has not been studied before. The aim of this study was to determine the effect of a checklist to prevent incidents with technical equipment problems during a routine laparoscopic procedure. Methods: A checklist was developed and tested during 20 laparoscopic cholecystectomies in a large non-university training hospital. A digital video capturing system was used, consisting of 3 camera image inputs and a microphone. The image of the endoscope, an overview of the operation room (OR), and an image of the hands of the surgeon were recorded. The microphone was placed near the equipment and the operation table. After the procedure the videos were analysed. Problems with technical equipment such as the insufflator, diathermy apparatus, monitors, light source, endoscope or instruments were registered and compared to the results of 30 laparoscopic cholecystectomies without checklist (control procedures)

Results: The total number of incidents per procedure was significant lower $(64 \%)$ than without the checklist (21/20 in contrast to 49/30). The number of incidents due to wrong positioning, setting or connection was $16 / 20$ with the checklist, and $35 / 30$ without the checklist $(65 \%$ reduction). The number of incidents due to a defect or faulty function was $5 / 21$ with checklist, and $14 / 35$ without (58\% reduction). In $65 \%$ (13/20) of the procedures with a checklist one or more incidents with the equipment occurred compared to $87 \%(26 / 30)$ in the control procedures. Median time to complete the checklist was 2.5 minutes (range 1-5,9 minutes) Conclusion: The pre-operative checklist is feasible and reduces problems with laparoscopic technical equipment in the operation room. Future research should aim on the implementation of pre-operative checklists for different procedures. The potential benefits of technical solutions for routinely use of checklists should be investigated.

\section{O130}

\section{LAPAROSCOPIC DONOR NEPHRECTOMY: A PLEA FOR THE RIGHT-SIDED APPROACH}

N.F.M. Kok, N. Wentink, I.P.J. Alwayn, T.C.K. Tran, W. Weimar, J.N.M. IJzermans

Erasmus Medical Center Rotterdam, Rotterdam, The Netherlands

Background: Many centers prefer open donor nephrectomy if the live donor's right kidney has to be removed. Right-sided laparoscopic donor nephrectomy (R-LDN) has been associated with short renal vessels and, consequentially, with a greater risk of renal vein thrombosis in the recipient. However, the anatomy of the right kidney poses some natural advantages including no venous branches of the renal vein and the position of the right kidney in the abdomen. In the present study we investigated the consequences of R-LDN on the outcomes of donors and recipients.

Methods: From May 2001 to January 2006 all donors who underwent laparoscopic kidney donation at our institution were enrolled in a prospective study. A researcher collected all intra-operative data in the operation room. The best kidney was always left to the donor. If pathology or a significant difference in size was absent, we chose whether to remove the right or the left kidney. If the right renal artery seemed to be long enough we preferred R-LDN to left-sided LDN (L-LDN).

Results: Eighty-three donors underwent L-LDN and 94 donors underwent R-LDN. Baseline characteristics including body mass index, multiplicity of renal veins and arteries and gender did not differ between groups. Ten procedures $(6 \%)$ were converted to open, all of them in the $L-L D N$ group $(P=0.001)$. Operation time was significantly longer for L-LDN (median 253 vs. 203 minutes) and blood loss was more (145 vs. $68 \mathrm{ml})$. Eighteen intra-operative complications (22\%) occurred during L-LDN and $9(10 \%)$ complications occurred during R-LDN $(P=0.03)$. Postoperatively, donors of both groups resumed a solid diet at the first day and were discharged after median 3 days. Postoperative complications occurred in $10(12 \%)$ and $4(4 \%)$ of the donors following L-LDN and $R$-LDN respectively $(P=0.09)$. These complications included 3 major complications in the L-LDN group (re-operation because of bleeding, open splenectomy and re-admission because of a wound infection) and one major complication in the R-LDN group (re-operation because of a bowel perforation). With regard to the recipients, venous thrombosis did not occur. Renal function and ureteral complication rate were similar for recipients of either a left or a right kidney.

Conclusion: Results of R-LDN were superior to results of L-LDN. RLDN should be expanded to allow all live kidney donors to benefit from the advantages of laparoscopic surgery.

\section{1}

\section{COST-UTILTY ANALYSIS OF LAPAROSCOPIC VERSUS MINI-INCI-} SION OPEN DONOR NEPHRECTOMY

N.F.M. Kok, N. Wentink, W. Weimar, T.C.K. Tran, I.P.J. Alwayn, J.N.M. IJzermans

Erasmus Medical Center Rotterdam, Rotterdam, The Netherlands

Background: Laparoscopic donor nephrectomy (LDN) is associated with higher in-hospital costs than open donor nephrectomy (ODN). The aim of the present study is to investigate the cost-efficiency of LDN. Methods: In a randomized controlled trial LDN was compared with miniincision muscle splitting ODN. Fifty donors were included in each arm. A cost-effectiveness analysis was conducted from a health care perspective as well as from a societal perspective, the latter including productivity losses. Use of health care resources during follow-up were registered using case record forms. Quality Adjusted Life Years (QALY's) were calculated from Euroqol-5D scores administered preoperatively and $3,7,14,28,90,180$ and 365 days postoperatively. Acceptability curves were derived using bootstrap analysis.

Results: Mean total costs were 6,090 Euros following LDN and 4,818 Euros following ODN $(P<0.001)$. These costs consisted of costs generated in the operation room (mean costs 4074 vs. 2244 Euros, LDN vs. ODN), hospitalization costs (1923 vs. 1496 Euros) and costs after hospitalization ( 521 vs. 652 Euros). Disposables (1871 vs. 533 Euros, LDN vs. ODN) and capital cost associated with endoscopic instruments (420 vs. 222 Euros) influenced the cost difference most. In either group thirty-four donors performed paid labour preoperatively. They returned to work after 68 and 75 days (LDN versus ODN) on average. Taking into account hours worked preoperatively, 36 additional working hours were lost per working donor In the ODN group on average. Thirty-six hours cost 1236 euros ( 36 hours $\times 80 \%$ x 40 euro per hour). Therefore, additional productivity losses per donor were 783 Euro following ODN $(34 / 50 \times 1236$ euro). The main gain in Quality of Life in the LDN group was realized within 4 weeks postoperatively. LDN resulted in a mean gain of 0.03 QALY at mean costs of 1271 and 488 Euro from respectively a healthcare perspective and a societal perspective. This implies that one additional QALY for the LDN group costs about 16,000 Euros more from a societal point of view and about 41,000 Euros from a health-care perspective.

Conclusion: LDN is, given a societal perspective, an efficient procedure mainly due to less productivity losses of donors.

\section{O132}

RANDOMIZED CONTROLLED TRIAL ON THE TRANSFER VALIDITY OF KNOT-TYING TRAINING IN VR SIMULATOR TO A LAPAROSCOPIC ENVIRONMENT

E.G.G. Verdaasdonk' ${ }^{1}$, J. Dankelman ${ }^{1}$, J.F. Lange ${ }^{2}$, L.P.S. Stassen ${ }^{3}$ 1 Delft University of Technology, Delft, The Netherlands

2 Erasmus Medical Center Rotterdam, Rotterdam, The Netherlands 3 Reinier de Graaf Groep Delft, Delft, The Netherlands 
Introduction: Laparoscopic intra-corporal suturing is one of the most difficult tasks in endoscopic surgery. It requires proficient eye-hand coordination and knowledge of the correct steps to tie the knot. The aim of this study was to determine the transfer validity of knot-tying training in a VR simulator to a realistic laparoscopic environment. Study design Randomised controlled trial

Methods: The knot-tying module of the SIMENDO® simulator was used. Twenty surgical trainees first underwent basic eye-hand coordination training until predefined competence criteria were met. Thereafter they were randomised into two groups. Group A received additional training with the knot tying module of the simulator and performed 10 times a double laparoscopic knot. Group B received no additional endoscopic hands-on training. Instead Group B watched 3 times a video demonstration of the knot-tying in the VR simulator. Within a week all participants performed a double knot in the abdominal cavity of an anaesthetized animal model. The procedure was captured on digital video. Videos were analysed; parameters studied were time to tie the knot and the number of predefined objective errors. Furthermore, two experienced laparoscopic surgeons, blinded to the training status of the trainees, also assessed the performance with global rating list on a 5 point Likert scale concerning economy of movement and erroneous behaviour.

Results: The trained group $A(n=9)$ was $30 \%$ faster than the control group $B(n=10)$, with a median of 262 versus 374 seconds $(p=0.034)$. The trained group also showed also a lower number of errors than the controls (median number of 24 versus 35 errors, $p=0.030$ ). The semiobjective assessment by the laparoscopic experts, however, showed no significant difference for economy of movement and erroneous behaviour between the groups.

Conclusion: Surgical residents receiving VR training in laparoscopic knot tying perform better in a realistic animal model compared to those who had not received specific simulation training. The studied VR mode is, therefore, a valid and useful tool for training of laparoscopic suturing. Furthermore, the study revealed opportunities for improvement of the simulator that could further enhance future training.

\section{O133}

CAN TRANSIT TIME FLOW MEASUREMENT PREDICT INTRA-OPERATIVE BYPASS FAILURE?

\section{Cikirikcioglu, S.O. Cruz, E. Pektok, A. Kalangos, B.H. Walpoth University Hospital of Geneva, Geneva, Switzerland}

Background and aim: Intra-operative flow measurement during peripheral bypass operations is important to rule out technical failures. For this reason, the flow measurement techniques should be validated. The aim of this study is to compare the accuracy of transit time flow measurement with angiography for diagnosing technical failure.

Materials and methods: Thirteen pigs underwent bilateral carotid artery interposition. Baseline intra-operative carotid artery flow was measured by a transit time flowmeter (Cardiomed, MediStim, Norway). After the graft interposition flow measurement was repeated, then a selective carotid angiography was performed via the common femoral artery. Quality control of the anastomoses was evaluated according to the modified NASCET formula by using angiographic images (Cikirikcioglu et al. Int J Artif Organs 2006; 29: 990-9). Technical failure was described when more than $50 \%$ stenosis in the quantified carotid angiography(QCA) was found. According to this information, the implanted grafts were grouped as technically normal or stenosed. Hemodynamic values, calculated with the rate-pressure product $(\mathrm{RPP}=$ mean arteriel blood pressure $\mathrm{X}$ heart rate) and measured flow of these two groups were then compared. Sensitivity and specifity values were calculated for transit time flow measurement.

Results: 4 of 26 interpositioned grafts showed more than $50 \%$ stenosis. The baseline hemodynamic and flow values were not different between the stenosed and normal groups(Table). The post-implantation hemodynamic values were also similar in both groups, but the flow was significantly lower in the technical failure group compared to the normal grafts. The sensitivity and specifity of transit time flow measurement were calculated as 100 and $86.3 \%$, respectively.

Conclusion: Transit time flow measurement was found to be a simple, safe and effective technique for a non-invasive, intra-operative quality control of possible the technical failure in this model. The sensitivity and specificity values showed that transit time flow measurement is a good method for diagnose technical failures and indicates using of further imaging techniques.

\begin{tabular}{|c|c|c|c|}
\hline & & $\mathrm{RPP}(\mathrm{mmHg} / \mathrm{min})$ & FLOW (ml/min) \\
\hline \multirow{2}{*}{$\begin{array}{c}\text { QCA stenosis }<50 \% \\
(\mathrm{n}=22)\end{array}$} & PREOP & $4402 \pm 1206$ & $209 \pm 56$ \\
\hline & POSTOP & $5241 \pm 1616$ & $192 \pm 87$ \\
\hline \multicolumn{2}{|l|}{$\mathbf{P}^{*}$} & 1.000 & 0.511 \\
\hline \multirow{2}{*}{$\begin{array}{c}\text { QCA stenosis }>51 \% \\
(n=4)\end{array}$} & PREOP & $4881 \pm 3112$ & $254 \pm 100$ \\
\hline & POSTOP & $5247 \pm 2457$ & $58 \pm 8$ \\
\hline \multicolumn{2}{|l|}{$\mathrm{P}^{\star}$} & 0.918 & $<0.001$ \\
\hline
\end{tabular}

*= Mann-Whitney U test

O134

ONLINE REGIONAL BLOOD FLOW MEASUREMENT -

CALIBRATION OF A NEAR INFRARED LASER DOPPLER DEVICE AND DETECTION OF CORONARY ARTERY BYPASS GRAFT OCCLUSION IN A PORCINE MODEL

B.O. Bierbach

University Hospitals Saarland, Homburg, Germany

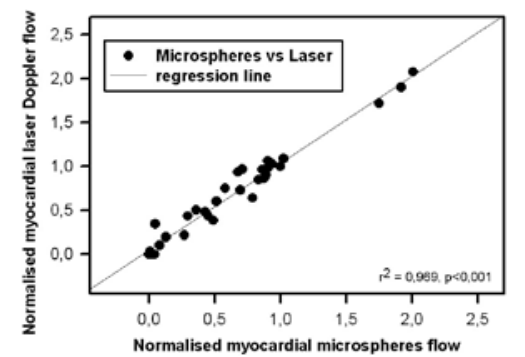

Correlation microspheres with laser Doppler flow

Objective: Detection of bypass graft occlusion after coronary artery bypass surgery poses a challenge in postoperative patient care. Until recently, there had been no reliable method that could detect regional myocardial blood flow (rMBF).

Methods: Therefore a laser Doppler (LEA-Medizintechnik, Giessen, Germany) was developed to measure rMBF. The laser was emitted during end diastole by blood pressure triggering. For calibration simultaneously to the laser measurements fluorescent $15 \mu \mathrm{m}$ microspheres were injected in the left atrium in 10 pigs. The laser Doppler probe was sutured to the anterior wall of the left ventricle. Blood flow in the LAD was measured by an ultrasonic flow meter. Blood flow reduction was achieved by a shaft driven occluder positioned around the LAD. At baseline and after reduction to 70 and $30 \%$ of the baseline value respectively myocardial blood flow was measured. The reduction level was kept constant for 10 minutes at a time. Then coronary occlusion was abolished. Regional myocardial blood flow determination was again performed 20 and 120 minutes later and finally after LAD occlusion.

In eight further pigs a left internal mammary artery to left anterior descending (LAD) coronary artery bypass was constructed during 60 minutes of cardiopulmonary bypass with 40 minutes aortic cross clamping. Cardioplegia was induced by warm potassium enriched blood. The LAD was ligated just proximal to the anastomosis, so myocardial blood flow distal to the ligation site was completely bypass dependent. In this area the laser Doppler probe was positioned. The myocardial blood flow and laser Doppler set-up was identically to the calibration experiments. Two hours after surgery the LIMA was occluded and a final rMBF determination was performed.

Results: Regional myocardial blood flow determined by microspheres showed a decrease during coronary occlusion, recovery during reperfusion and no flow during $L A D$ occlusion (baseline:76,6 $\pm 13,0$; $70 \%: 61,7 \pm 7,5 ; 30 \%: 11,3 \pm 2,7 ; 20$ minutes:69,2 $\pm 10,9 ; 120$ minutes:57,9 $\pm 10,1$; occlusion:0,5 $\pm 0,3[\mathrm{ml} / \mathrm{min} \times 100 \mathrm{~g}])$. Laser Doppler measurements normalised to baseline values strongly correlated with the microspheres' data $(r 2=0,969, p<0,001)$.

Regional myocardial blood flow in the coronary artery bypass model showed same baseline values as in the calibration experiments. After release of the aortic cross clamp during early reperfusion reactive hyperaemia was detected and regional myocardial blood flow returned to baseline values during the following 2 hours of reperfusion. Bypass occlusion led to break-down of blood flow in the bypass dependent area measured with both blood flow determination methods. The bypass occlusion was online detected by the laser Doppler probe immediately. Conclusion: For the first time a laser Doppler probe provides online information about regional myocardial blood flow in a porcine model. 
Due to the nature of laser Doppler flowmetry there are no concrete values obtainable instead of relative changes, but complete breakdown in regional myocardial blood flow can be reliably online detected.

0135

EFFECT OF RECOMBINANT APROTININ ON POSTOPERATIVE BLOOD LOSS AND CORONARY VASCULAR FUNCTION IN A CANINE MODEL OF CARDIOPULMONARY BYPASS

G. Veres ${ }^{1,2}$ T. Radovits ${ }^{1,2}$, H. Schultaz ${ }^{1}$, L. Lin ${ }^{1}$ J. Hütter ${ }^{3}$, E. Weigang ${ }^{4}$, Z. Szabolcs ${ }^{2}$, G. Szabó

1 Department of Cardiac Surgery, University of Heidelberg, Heidelberg, Germany

2 Department of Cardiovascular Surgery, Semmelweis University, Budapest, Hungary

3 Bayer Health Care, Wuppertal, Germany

4 Department of Cardiovascular Surgery, University of Freiburg, Germany

Objectives: Aprotinin is a widely used serine protease inhibitor during cardiopulmonary bypass to reduce blood loss and preserve platelet function. However, the bovine-derived aprotinin can induce hypersensitivity reaction with fatal complications. Furthermore, vascular effects of aprotinin are not completely elucidated. The current study is designed to investigate the effects of recently developed recombinant aprotinin on blood loss and coronary vascular function in a clinically relevant canine model of cardiopulmonary bypass.

Methods: 24 dogs underwent cardiopulmonary bypass. Dogs were divided into three groups in a blinded fashion: control animals $(n=8)$ received placebo, aprotinin treatment groups received bovine $(n=8)$ or recombinant aprotinin $(n=8)$ according to Hammersmith method. The doses of bovine and recombinant aprotinin were the same. Coagulation parameters and blood loss was measured regularly at different time points. Endothelium-dependent and -independent vasorelaxation were investigated in isolated left anterior descendent coronary arterial rings by using acetylcholine and bradykinin or sodium nitroprusside and adenosine, respectively.

Results: Postoperative blood loss was significantly reduced in the aprotinin-treated groups in comparison to control and there was no significant difference between the two aprotinin-treated groups. Endothelium-dependent relaxation of coronary arteries to acetylcholine and bradykinin was unaffected in the aprotinin treatment groups. Both types of aprotinin significantly increased vasorelaxation to adenosine when compared with controls, but not affected that to sodium nitroprusside. Conclusion: Effectiveness of recombinant aprotinin on blood loss was equivalent with bovine-derived aprotinin. Neither types of aprotinin impaired endothelium-dependent relaxation in a canine model of cardiopulmonary bypass.

\section{6}

DEVELOPMENT OF A NOVEL MODEL FOR KIDNEY TRANSPLANTATION IN THE RAT: PILOT-STUDY OF ISOLATED RAT URETER CANNULATION AND UNRESTRICTED POST-OPERATIVE SURVIVAL

C. Moers ${ }^{1}$, A. Smit-van Oosten², J.H. Zandvoort ${ }^{2}$, H.G.D. Leuvenink ${ }^{1}$, R.J. Ploeg

1 UMC Groningen, Groningen, The Netherlands

2 University of Groningen, Groningen, The Netherlands

Introduction: The most commonly used rat model to study short term graft function after kidney transplantation is orthotopic transplantation with immediate contralateral nephrectomy in the recipient. Since the recipient animal's kidney function will be fully dependent on the graft, this model is best suited for investigating graft function directly after transplantation. However, when interventions are to be studied that substantially damage the donor kidney, such as prolonged warm ischemia, delayed graft function (DGF) will occur. Since a rat cannot be easily dialyzed, most recipients will die as a result of DGF. Leaving the contralateral kidney in situ would keep the recipient animal alive, but then short term graft function cannot be assesed. This could be resolved by cannulating the graft ureter and continuously collect isolated graft urine while the native contralateral kidney remains in situ. This approach has never been followed before. As a first step towards developing such a model, we have conducted a pilot-study to investigate whether it is technically possible to cannulate one ureter of a healthy, untransplanted rat and continuously collect urine from this kidney while the animal is conscious and unrestricted.
Methods:In nine male Lewis rats one ureter was cannulated under general anesthesia via a median laparotomy. The cannula was tunneled subcutaneously from the abdomen to the head, fixed to the skull and connected to a swivelling tube construction by which the animal could move freely. Urine was continuously collected via this tubing. Animals were allowed to survive for at least one week.

Results: After a short learning curve, a reliable method for cannulating a rat ureter and properly fixating the cannula was obtained. All animals survived the operation. In eight animals there was immediate and prolonged urine production via the swivelling tube. Most animals survived beyond the one week period. Hydronephrosis of the cannulated kidney was seen only when prolonged stasis of urine in the system had occurred by accident due to air entrapment. Spontaneous occlusion of the ureter cannula or tubing was never observed.

Conclusion: Cannulation of one rat ureter and continuous extracorporeal urine collection while the animal is conscious and unrestricted is technically possible. This method will be further perfected and incorporated into a kidney transplant model. More results will be available at the time of the ESSR congress.

\section{O137}

DONOR DNA IS DETECTED IN RECIPIENT BLOOD FOR YEARS AFTER KIDNEY TRANSPLANTATION ITS ROLE IN REJECTION OR ACCEPTANCE?

J. Rutkowska1 , B. Interewicz' ${ }^{1}$ A. Rydzewski ${ }^{1}$, M. Swiatek ${ }^{2}$,

A. Dominiak', M .Durlik¹, W.L. Olszewski ${ }^{3}$

1 Medical Research Center, Warsaw, Poland

2 Central Clinical Hospital, Warsaw, Poland

3 The Norwegian Radium Hospital, OSLO, Norway

After organ transplantation passenger cells and cellular debris from ischemia and rejection damaged cells disseminate in recipient and in most part are being taken up by macrophages and dendritic cells (DC). In long surviving grafts immunosuppressive drugs also damage graft cells and their DNA is spread in the recipient. The most important question is whether donor DNA may be incorporated into recipient cell genome in the so called 'illegitimate DNA incorporation' a frequent phenomenon in nature. A subsequent question would be the presumptive effect of donor DNA on the rejection process.

Aim: To search for donor DNA in recipient tissues and cells after allogeneic transplantation and immunosuppression.

Materials: Recipient's blood samples were collected in the following time point sequences: before and after transplantation on day 1, 14, 28, 90 and 180 days and 1 and 2 years. Genomic DNA was isolated from donor's spleen and recipient's blood using NucleoSpin Kit (MachereyNagel). Quantification of DNA was performed spectrophotometrically in capillary cuvettes on GeneQuant (Amersham Pharmacia Biotech). Quality of isolated DNA was checked electrophoretically on $1 \%$ agarose gel with ethidium bromide. To identify donors' genetic material in the recipient's blood short tandem repeats analysis was applied (STR). The following loci were investigated: phospholipase A2- HUMPLA2A1(AAT)n, cytochrome P450 HUMCYARO(AAAT)n and D1S80. This type analysis is used in forensic medicine. PCR reaction mixture contained $25 \mathrm{ng}$ DNA, $1 U$ Fast Start Taq DNA Polymerase (Roche), 15 pmol fluorescent (Cy-5) oligonucleotide primers. The PCR amplification was carried out in a Thermal Cycler (MJ Research). The PCR products was analysed by electrophoresis in 6\% PAGE using ALFExpress sequencer (Amersham Pharmacia Biotech). The length of fragments in comparison with internal and external standards was identified using Fragment Manager program.

Results: Donor cytochrome P450 or HUMPLA2 or D1S80 genes were detected in recipient blood cells up to 2 years after kidney transplantation. Positive results were observed already $24 \mathrm{hr}$ after grafting (3pts) and in 4 pts after 14 days, in 28 after 28, in 2 after 90, in 3 after 180, in 1 after 1 and in 2 after 2 years. All patients remain under further follow-up. The question as to whether donor DNA was contained in donor cells or as apoptotic or necrotic bodies in recipient phagocytes remains to be answered.

Conclusion: Donor DNA can be detected in recipient blood cells 2 years after kidney transplantation. Its role in rejection or acceptance is not clear, although we know from animal experiments that it is detected in recipient dendritic APCs (antigen presenting cells) 
0138

HYDROGEN SULFIDE REDUCES ENERGY STATUS IN THE ISOLATED PERFUSED KIDNEY, RENAL TUBULAR CELLS AND ISOLATED MITOCHONDRIA

E.M. Bos, H. Van Goor, H.G.D. Leuvenink

University Medical Center Groningen, Groningen, The Netherlands

Introduction: Hydrogen Sulfide (H2S) is a toxic gas that exerts strong metabolic effects. It has recently been discovered that $\mathrm{H} 2 \mathrm{~S}$ administered to mice in subtoxic concentrations induces a reversible, hibernationlike state. During $\mathrm{H} 2 \mathrm{~S}$ treatment, the core body temperature declined to $\sim 2{ }^{\circ} \mathrm{C}$ above ambient and CO2-output and O2-uptake went to $\sim 10 \%$ of basal levels. Cessation of $\mathrm{H} 2 \mathrm{~S}$ administration caused the physiologic metabolic state of the animal to return, without signs of damage or toxicity. These findings signify $\mathrm{H} 2 \mathrm{~S}$ as a candidate for the reduction of renal energy demand during stressful conditions, such as ischemia/reperfusion. To validate this assumption, we studied the effects of $\mathrm{H} 2 \mathrm{~S}$ on the metabolic state in isolated kidneys, cultured renal tubular cells and isolated mitochondria.

Materials and methods: Rat kidneys were placed in an Isolated Perfused Kidney (IPK) setup and perfused with oxygenated perfusion medium at $37^{\circ} \mathrm{C}$. NaHS (a donor of $\mathrm{H} 2 \mathrm{~S}$ ) was added to the perfusate, and differences between pre- and postrenal oxygen concentration were measured before and after $\mathrm{NaHS}$ addition. Also, perfusion pressure was continuously monitored. Tissue samples were taken to determine total renal ATP. For cell studies, NRK-52E renal tubular cells were cultured in 48-well plates, and loaded with the fluorescent mitochondrial membrane potential probe JC-1. Cells were exposed to different concentrations of $\mathrm{NaHS}$ and fluorescence was measured. Afterwards, NaHS was washed out ant membrane potential recovery analyzed. In mitochondria isolated from rat liver, oxygen use was measured using a Clark-type oxygen electrode. Also, JC-1 fluorescence and ATP production were determined.

Results: In the IPK setup, addition of NaHS reduced renal oxygen uptake by $65 \%$ and total ATP content by $50 \%$. NaHS reduced a phenylephrine-induced increase in perfusion pressure by $32 \%$. In NRK cells, mitochondrial membrane potential was decreased from $0.5 \mathrm{mM}$ $\mathrm{NaHS}$ and upwards, reaching a maximum of $\sim 50 \%$ reduction at $3 \mathrm{mM}$. The reduction in membrane potential was reversible to pre-treatment levels at all concentrations. In isolated mitochondria, NaHS completely inhibited oxygen use, and abolished ATP production and mitochondrial membrane potential.

Conclusion: $\mathrm{H} 2 \mathrm{~S}$ lowers the energy demand of the isolated kidney, and causes extensive vasodilatation. In cell culture, H2S results in a reversible mitochondrial function loss. This method of metabolic inhibition might be suitable as a protective intervention against ischemia/reperfusion injury. Treatment with subtoxic concentrations of $\mathrm{H} 2 \mathrm{~S}$ prior to renal graft harvest might prevent hypoxia-related organ damage.

\section{9 \\ ECTOIN AMELIORATES ISCHEMIA/REPERFUSION INJURY OF COLD STORED SMALL BOWEL GRAFTS}

W.L.I. Wei ${ }^{1}$, K. Hata ${ }^{2}$, B. Doorschodt ${ }^{3}$, R. Bttner ${ }^{2}$, T. Minor ${ }^{2}$, G. van Echten Deckert ${ }^{2}$, R.H. Tolba ${ }^{4}$

1 University of Bonn Medical Center, Bonn, Germany

2 University of Bonn, Bonn, Germany

3 University of Amsterdam, Germany

4 House of Experimental Therapy, University, Bonn, Germany

Purpose: Ectoin is a natural substance, discovered in extreme halophilic bacteria. It stabilizes proteins and other cellular structures and protects the skin from stresses like UV irradiation and dryness. The aim of the present study was to evaluate the potential of Ectoin in the cold preservation of small bowel (SB) grafts.

Material and methods: Male Wistar rats were used as donors and the small bowel $(n=6)$ were retrieved and thereafter flushed with $10 \mathrm{ml}$ Polysol (group P) or 10ml Polysol with 10mg Ectoin (group E) and then stored in the respective solution for 18 hours at $4^{\circ} \mathrm{C}$. Post-ischemic reperfusion was performed at constant flow $(8 \mathrm{ml} / \mathrm{min})$ in a recirculating system, with oxygenated $(95 \% 02 \& 5 \%$ C02) modified Krebs-HenseleitBuffer at $37^{\circ} \mathrm{C}$. Results (P vs. E; Mean \pm SEM; * $=p<0.05$ ): Structural integrity during reoxygenation turned out to be superior with the addition of Ectoin to the preservation solution and group $E$ showed the lowest values of parenchymal enzyme release (LDH: $54.5 \pm 21$ vs. $25.4 \pm 8.3$
$\mathrm{U} / \mathrm{I}$ ); Cellular damage of the small bowel during ischemia and reperfusion , e.g apoptosis ( $29.2 \pm 3.3$ vs. $22.9 \pm 1.4 \mathrm{au})$, MDA release $(0.42$ \pm 0.03 vs. $0.35 \pm 0.02 \mathrm{nmol} / \mathrm{ml})$ and $\mathrm{NO}$ release $(0.79 \pm 0.13$ vs. $0.33 \pm$ $0.21 \mathrm{nmol} / \mathrm{l}$ ) were also significant lower in group $\mathrm{E}$. Mucosal absorptive function was assessed by uptake of galactose from the intestinal lumen to the portal vein effluent. Here group $E$ showed highest values in comparison to the group P (Galactose: $92.0 \pm 31.2$ vs. $48.5 \pm 29.4 \mathrm{mg} \%$ ). It is concluded from these results that Ectoin improved the preservation of cold stored small bowel graft and ameliorated the ischemia/reperfusion injury. Further studies with Ectoin in vivo are strongly encouraged.

\section{O140}

RECOMBINANT HUMAN ANTITHROMBIN PREVENTS THE INFLAMMATORY BLOOD MEDIATED REACTION AFTER INTRAPORTAL ISLET ALLOGRAFT

R. Caiazzo ${ }^{1}$, L. Arnalsteen ${ }^{2}$, M. Jourdain ${ }^{2}$, T. Jany², A. Tournoys ${ }^{2}$, V. Gmyr ${ }^{2}$, T. Hubert ${ }^{2}$, P. Gosset ${ }^{2}$, J. Kerr-Conte ${ }^{2}$, F. Pattou ${ }^{2}$ 1 Inserm U859, Lille University Hospital, Lille, France, France

The injection of allogenic islet preparations into the portal vein activates the coagulation cascade and provokes an intense inflammatory reaction, two deleterious events for the graft and the function of the islets. We studied the effects of recombinant antithrombin (rhAT), an anticoagulant molecule with antiinflammatory properties previously proposed in other situations associated with acute thrombosis and inflammation.

Methods: Systemic markers of haemostasis, endothelial aggression and inflammation were measured in non diabetic pigs $(n=29$, Large White, $14-18 \mathrm{~kg}$ ) for 24 hours after injection of 0.15 (groups 1, 2 or 3 ) or $0.3 \mathrm{ml} / \mathrm{kg}$ (groups A or B) of an allogenic islet preparation. Islets, isolated with a modified Ricordi's automated method using porcine liberase were injected in Ringer's lactate and porcin albumin (0.1\%). Animals received no treatment (groups 1 or A) or treatment with $1000 \mathrm{UI} / \mathrm{kg}$ of rhAT (groups 2 or B) or $25 \mathrm{UI} / \mathrm{kg}$ of heparin (group 3 ).

Results: After the injection of $0.15 \mathrm{ml} / \mathrm{kg}$, the administration of rhAT (group 2) improved the coagulation markers (reduction in the fall of leukocytes [p<0.01 at 30min, group 2 vs 1], of fibrinogen, and Quick's time [ $p<0.05$ at 2 hours, group 2 vs 1], and inflammation (reduction of the peak of TNFalpha [p<0.01 at 1 hour, group 2 vs 1], of IL6, and IL10 [ $p<0.05$ at 1 hour, group 2 vs 1]), and endothelial lesions (reduction of the increase in von Willebrand factor [ $p<0.05$ at 2 hours, group 2 vs 1]) This anti-inflammatory effect was not found in group 3 which received heparin. After injection of an extreme volume of islet preparation $(0.3$ $\mathrm{ml} / \mathrm{kg}$ ), administration of rhAT reduced the mortality from 4/4 (group A) to $1 / 5$ (group $B, p<0.05$ vs $A$ ).

Conclusion: Adjuvant treatment by rhAT prevents the activation of the coagulation and inflammatory cascades. If the control of these initial reactions proves beneficial for the function of the islets, treatment with rhAT should be an interesting clinical prospect.

\section{O141}

\section{TRANSPLANTATION OF NEO-HEPATOCYTES IN FIBROTIC MICE} LIVERS

C. Eipel ${ }^{1}$, A.K. Nuessler ${ }^{2}$, K. Sievert ${ }^{1}$, M. Brulport ${ }^{3}$, J.G. Hengstler ${ }^{3}$, B. Vollmar ${ }^{1}$

1 University of Rostock, Rostock, Germany

2 Fresenius Biotech, Bad Homburg, Germany

3 University of Dortmund, Dortmund, Germany

Introduction: Hepatocyte transplantation has been successful in many animal models of acute liver failure. In contrast, only few clinical attempts have been made, mainly because of the lack of a reliable source of liver cells. Transplantation of human stem or precursor cells may represent an alternative technique. Herein, we evaluated in an experimental animal model whether transplantation of Neo-Hepatocytes (NeoHeps), i.e. hepatocyte-like cells differentiated from blood monocytes, could sustain function and recovery of fibrotic liver tissue.

Materials and methods: For induction of liver fibrosis 26 male mice (NOD.CB17-Prkdscid/J) received an intraperitoneal application of $\mathrm{CCl} 4$ ( $2 \mathrm{ml} / \mathrm{kg}$ bw, 1:4 in corn oil) twice a week for 6 weeks. To evaluate the extent of fibrosis animals were killed at the 4th day after last administration of $\mathrm{CCl} 4$ and blood as well as liver tissue samples were taken for subsequent analysis (Fib $n=6)$. At the 4th day after last $\mathrm{CCl} 4$ administration additional animals received either a suspension of $\mathrm{NeoHeps}(1 \times 106$ cells/mouse in $100 \mu$ isotonic $\mathrm{NaCl}$, FibNeo) or $100 \mu$ isotonic $\mathrm{NaCl}$ 
alone (FibCon) in the left lateral lobe of the liver. Time points of evaluation were as follows: day 2 puncture of venous retrobulbar plexus under light ether anaesthesia for immediate separation of EDTA plasma $(n=4$ per group); day 7 ( $n=4$ per group) and day 21 ( $n=6$ per group) exsanguination and asservation of plasma and of the left lateral hepatic lobe for subsequent analysis. Blood and liver tissue samples of untreated animals served for assessment of physiological parameters (Con $n=6$ ). Means \pm SEM. ANOVA and post-hoc-comparison ( ${ }^{*} p<0.05$ vs. Con). Results: At the 4th day after last administration of $\mathrm{CCl} 4$ liver sections revealed fibrous septa around the centrilobular veins with an almost disclosing pattern of these septa dividing the liver parenchyma into multiple discrete nodules. In line with this, ALT plasma level was found significantly increased when compared to untreated controls (U/l; Fib vs. Con: $81 \pm 16^{*}$ vs. $\left.16 \pm 2\right)$ and synthesis of urea was significantly decreased (mg/dl; Fib vs. Con: $50 \pm 3^{*}$ vs. $54 \pm 2$ ) accompanied by an elevated plasma concentration of ammonia ( $\mu \mathrm{mol} / \mathrm{l} ;$ Fib vs. Con: $168 \pm 77^{*}$ vs. $82 \pm 24)$. Transplantation of NeoHeps did not improve liver function compared to sham treated animals with fibrosis (urea mg/dl; FibNeo vs. FibCon, 2 d: $41 \pm 1$ vs. $39 \pm 2$; 7 d: $42 \pm 3$ vs. $44 \pm 2$; 21 d: $57 \pm 2$ vs. $58 \pm 2$ ). Plasma ammonia levels decreased without any differences between groups ( $\mu \mathrm{mol} / /$; FibNeo vs. FibCon, $2 \mathrm{~d}$ : $173 \pm 14$ vs. $156 \pm 3$; $7 \mathrm{~d}$ : $136 \pm 14$ vs. $147 \pm 21 ; 21 \mathrm{~d}$ : $53 \pm 8$ vs. $65 \pm 4$ ). Transplantation of NeoHeps did not led to a faster recovery of liver injury (ALT U/l; FibNeo vs. FibCon, 7d: $41 \pm 4$ vs. $35 \pm 5$; 21 d: $26 \pm 6$ vs. $35 \pm 12$ )

Conclusion: Intrahepatic transplantation of $\mathrm{NeoHeps}$ did ' at least in a murine model of chronic $\mathrm{CCl} 4$-intoxication - not support liver function as well as liver recovery. Further studies are necessary to show to which extent other modes of application, i.e. injection into the portal vein or into the spleen, could lead to an integration and function of NeoHeps. So far, transplantation of progenitor cells seems to be unsuitable in case of chronic liver failure.

\section{2}

THE ROLE OF ADENOSINE-A2 STIMULATION IN THE PROTECTION OF STEATOTIC LIVER GRAFTS DURING COLD STORAGE

K.H. Hata' , L. Wei' ${ }^{1}$ Y. Yamamoto ${ }^{2}$, R. Buettner ${ }^{1}$, T. Minor ${ }^{1}$, R. h. Tolba 1 University of Bonn, Bonn, Germany

2 Akita University School of Medicine, Akita, Japan

Background: Current drastic shortage of donor organs, coupled with an increasing incidence of hepatic steatosis, have led to acceptance of steatotic donor livers for transplantation, despite a higher risk of primary graft dysfunction. We herein report the effect of CGS-21680, a selective adenosine-A2 agonist, on cold storage of steatotic liver grafts in a rat model of dietary hepatic steatosis.

Methods: Hepatic steatosis was induced in male Wistar rats (240-270 g) by 2-day fasting and subsequent 3-day re-feeding with a fat-free, carbohydrate-rich diet. This protocol induced predominantly macrovesicular steatosis, which was confirmed by Sudan red staining. Fatty livers were retrieved, flushed and stored at $4^{\circ} \mathrm{C}$ for 24 hours using HTK, with or without CGS supplementation of $30 \mathrm{mg} / 100 \mathrm{ml}$ (group A and group C, respectively). Functional integrity of the graft was thereafter evaluated by isolated reperfusion with oxygenated Krebs-Henseleit buffer at $37^{\circ} \mathrm{C}$ for 45 minutes in the both groups.

Results: (group C vs. group A, mean \pm SEM in 7 dependent observations) CGS supplementation resulted in significant reduction of AST, ALT and $L D H$ release (ALT at $45 \mathrm{~min}$.; $2301 \pm 185 \mathrm{IU} / \mathrm{l}$ in group $\mathrm{C}$ vs. 1620 \pm 216 in group $A ; p<0.05$ ), whereas intramitochondrial enzyme GLDH release was not different (at $45 \mathrm{~min}$.; $2961 \pm 707 \mathrm{IU} / \mathrm{l}$ vs. $2376 \pm 265$; $p=0.451$ ). Portal venous pressure (PVP at $45 \mathrm{~min}$.; $16.89 \pm 2.69 \mathrm{mmHg}$ vs. $13.78 \pm 1.74 ; p>0.05$ ), hepatic $\mathrm{O} 2$ consumption (at $45 \mathrm{~min}$.; $0.2907 \pm$ $0.0465 \mathrm{mmol} / \mathrm{g}$ liver $/ \mathrm{min}$. vs. $0.4606 \pm 0.0913 ; p>0.05$ ), tissue ATP concentration $(0.736 \pm 0.093 \mathrm{mmol} / \mathrm{g}$ dry-liver vs. $0.806 \pm 0.042, \mathrm{p}=0.51)$, lipid peroxidation (malondialdehyde release into the perfusate at $45 \mathrm{~min}$; $1.922 \pm 0.198 \mathrm{nmol} / \mathrm{l}$ vs. $1.979 \pm 0.236, \mathrm{p}=0.856$ ) and tissue wet/dryweight ratio (5.20 \pm 0.31 vs. $4.88 \pm 0.13, p=0.357)$ showed no or less significant benefits. Electron microscopy also revealed insufficient protection of hepatic mitochondria by CGS. In order to clarify the possible mechanisms underlying these insufficient benefits, we focused on nitric oxide (NO) release, a well-known pivotal cascade following adenosineA2 stimulation. NO measurement in the effluent perfusate disclosed no significant difference between the both groups $(1.691 \pm 0.362 \mathrm{mmol} / / \mathrm{g}$ liver in group $C$ vs. $1.405 \pm 0.231$ in group $A ; p=0.507$ ), however, both of which reached up to 40 -fold higher level than in non-steatotic livers ( $0.037 \pm 0.005 \mathrm{mmol} / / / \mathrm{g}$ liver; $p<0.001$ vs. the both groups), suggesting 'shear-stress' mediated eNOS up-regulation in steatotic livers. The correlation analysis between nitric oxide (NO) release and PVP indicated 1) NO concentrations were in good correlation to PVP (Pearson $r=0.990$, $p=0.010, r 2=0.980)$. 2) NO up-regulation by CGS, a key trigger to subsequent protection, was substantially masked by concurrent $\mathrm{NO}$ upregulation in macrovesicular steatotic livers.

Conclusion: Adenosine-A2 stimulation using CGS-21680 resulted in limited benefits on the integrity and function of steatotic liver grafts, presumably due to the simultaneous NO up-regulation from shear stress in macrovesicular steatosis, thereby offsetting the expected benefits through adenosine-A2 stimulation.

\section{O143}

\section{SACRAL NEUROMODULATION IN PATIENTS WITH FAECAL} INCONTINENCE:

\section{RESULTS OF THE FIRST 100 PERMANENT IMPLANTATIONS}

\section{J. Melenhorst ${ }^{1}$, S. Koch ${ }^{2}$, O. Uludag ${ }^{3}$, W. van Gemert ${ }^{1}$, C. Baeten} 1 Academic Hospital Maastricht, Maastricht, The Netherlands

2 Viecuri Hospital, Venlo, The Netherlands

3 Atrium Medical Center, Heerlen, The Netherlands

Objective: Faecal incontinence is a socially devastating problem. Sacral Nerve Modulation (SNM) has proven its place in the treatment algorithm for patients with faecal incontinence. In this study the first one hundred definitive SNM implants in patients with faecal incontinence are evaluated.

Patients and methods: In a prospective single centre study patients were included between March 2000 and May 2005. Faecal incontinence was defined as at least one episode of involuntary faecal loss per week confirmed by a 3-week bowel habit diary. Patients were eligible for implant of a definitive SNM when showing at least a $50 \%$ reduction in incontinence episodes or days during ambulatory test stimulation. Preoperative workup consisted of a X-defecography, PNTML measurement, endo-anal ultrasound and anal manometry. The follow-up visits for the permanent implanted patients were scheduled at 1, 3, 6, and 12 months and annually thereafter. The bowel habit diary and anal manometry were repeated postoperatively during the follow-up visits.

Results: One hundred and thirty four patients were included and received a subchronic test stimulation. 100 patients $(74.6 \%)$ had a positive test stimulation and received a definitive SNM implantation. The permanent implantation group consisted of 89 women and 11 men. The mean age was 55 (26-75) years. The mean follow-up was 25.5 (2.5-63.2) months. The mean number of incontinence episodes decreased significantly during the test stimulation (baseline: 31.3 , test $4.4 ; p<0.0001$ ) and at follow-up, (36 months postoperatively $4.8 ; p<0.0001$ ). There was no significant change in the mean anal resting pressure. The squeeze pressures were significantly higher at six months $(109.8 \mathrm{~mm} \mathrm{Hg}, p=0.03), 12$ months $(114.1 \mathrm{~mm} \mathrm{Hg}, p=0.02)$ and 24 months postoperatively $(113.5 \mathrm{~mm} \mathrm{Hg}$, $\mathrm{p}=0.007)$. The first sensation, urge and maximum tolerable volume did not change significantly. 21 Patients were considered late failures and received further treatment.

Conclusion: Sacral neuromodulation is an effective treatment for faecal incontinence. The medium term results are satisfying.

\section{O144}

\section{IMPACT OF DIFFERENT TEXITURE OF POLYPROPYLENE MESH} ON THE INFLAMMATORY RESPONSE

\author{
G. Di Vita, R. Patti, S. Buscemi, M. Arcara, V. Bondi, F. Gioe \\ University of Palermo, Palermo, Italy
}

Background and aim: Over the past decade, hernia surgery has undegone a considerable transformation with the use of prosthetic materials. Polypropilene meshes that are most used, induces a rapid and usefull acute inflammatory response followed by chronic foreign body reaction. Many factors influence this response like density, size, physical characteristics, different texture and porosity of each biomaterials. Aim of this study was to assess if the implant of monofilament or multifilament meshes, in the inguinal hernioplasty, determine a different inflammatory response. Patients and methods: Thirty-two male patients were included in the study and randomly divided in two groups. In the first group (MO) inguinal hernioplasty was performed using monofilament polypropylene mesh, while in the second one (MU) multifilament prosthesis. Peripheral venous blood samples were collected 24 hours before surgery and then $6,24,48$ and 168 hours postoperatively.Modifications in leukocytes 
count, CRP, alpha-1 antitrypsin, interleukin (IL)-1, IL-6, IL-1 ra and IL-10 serum levels were recordered at all sampling times.

Results: We present evidence that serum levels of CRP, alpha-1 antitrypsin, leukocytes and all cytokines were significantly increased postoperatively in both groups, returning to basal values 168 hours afterwards. In particular, the production of all pro-inflammatory mediators was higher in MU group, whereas the antiinflammatory cytokine (IL-10, IL-1ra) production was higher in MO patients.

Conclusion: Although our results indicate that the polypropylene multifilament mesh allows an higher intense acute inflammatory response as compared to the monofilament mesh implantation and determine the formation of a strong scar tissue reducing the incidence of recurrences, to date, the optimal mesh is not present because an esagerate strong scar tisuue could be responsible of some complications.

\section{5}

\section{NNIS RISK INDEX}

M.C.F.Cainzos Fernandez ${ }^{1}$, F.J.G.R. Javier ${ }^{2}$

1 Hospital Clinico Universitario, Santiago de Compostela, Spain

Background: it has been reported some problems with the power of the National Nosocomial Infection Surveillance (NNIS) risk index to predict the risk of surgical site infections (SSI) for specific procedures.

Objectives: To develop an alternative risk prediction index for SSI and to compare the performance with the NNIS index.

Methods: A prospective cohort study was carried out with all (1596) patients submitted to digestive tract surgery in Hospital Clinico Universitario of Santiago de Compostela, Spain, from September 2005 through August 2006, the patients were followed-up for 30 days after surgery. Results: The final incidence rate of SSI was $12.7 \%$; 204 cases of SSI were identified. Two logistic multivariate analyses were used for construction of the model. Obesity $(p=0.017)$, surgery risk $(p=0.000)$, adjusted duration $(p=0.003)$, chronic obstructive pulmonary disease $(p=0.004)$, type of incision $(p=0.000)$, duration of preoperative bed stay $(p=0.000)$, colostomy $(p=0.006)$ a wound class classified as contaminated or dirty $(p=0.000)$, anticoagulation therapy $(p=0.001)$ and video laparoscopic surgery $(p=0.005)$ were statistically significant. The performance of the NNIS model in this study showed a low predictive capacity for the occurrence of SSI as determined by the receiver operating characteristic (ROC) curve $(0.531 ; 95 \% \mathrm{Cl}$ : 0.516-0.624) compared with the alternative model developed with this population $(0.832 ; 95 \% \mathrm{Cl}$ : $0.715-0.879$ ).

Conclusion: The presence of obesity, adjusted duration, and surgery risk, chronic obstructive pulmonary disease and anticoagulation therapy significantly increased the risk for SSI. The NNIS risk index was not significant for SSI in the sample studied, laparoscopic access, transverse incisions and short of preoperative bed stay was associated with a significant reduction in the risk for SSI. Although the NNIS index is a well-known and simple index, other models depicting variables related to SSI with a better sensitivity and specificity can be developed. Additional studies are required to confirm our results.

\section{6}

DOES THE ROUTE OF ADMINISTRATION (ENTERAL OR PARENTERAL) OF ISOTOPICALLY LABELED L-GLUTAMINE AFFECT THE CONVERSION OF L-[2-15N]GLUTAMINE INTO L-[2-15N]ARGININE

\section{IN HUMANS}

G.C. Ligthart-Melis ${ }^{1}$, C.G. van der Poll ${ }^{2}$, C.H.C. de Jong ${ }^{2}$, P.G. Boelens ${ }^{3}$, N.E.P. Deutz ${ }^{4}$, P.A.M. van Leeuwen ${ }^{1}$

1 VUMC Amsterdam, Amsterdam, The Netherlands

2 University Hospital Maastricht, Maastricht, The Netherlands

3 Catharina Hospital Eindhoven, Eindhoven, The Netherlands

4 UAMS, Center Trans.Res, Little Rock, USA, Little Rock, USA

Background and aims: L-glutamine and L-arginine exhibit numerous beneficial effects in experimental and clinical studies. A pathway of $\mathrm{L}$-glutamine into L-citrulline and L-arginine, involving intestinal and renal site-specific enzymes, has been suggested in the literature. The present study was designed to establish the effect of the feeding route, enteral or intravenous, on the conversion of L-glutamine into L-arginine at organ level in humans.

Methods: Sixteen patients undergoing upper gastrointestinal surgery received an intravenous or enteral infusion of $L-[2-15 N]$ glutamine $(15.7$ $\pm 0.7 \mu \mathrm{mol} / \mathrm{kg} / \mathrm{hr}$ ). Blood was sampled from a radial artery and from the portal and right renal vein. Amino acid concentrations and enrichments were measured and net fluxes of $15 \mathrm{~N}$ labeled substrates across the portal drained viscera (PDV) and kidneys were calculated (([A]-[V])* plasma flow). Results are expressed in mean \pm SEM. Differences between the intravenous (IV) and enteral group (EN) were tested using Student's t-test. A p-value $<0.05$ was considered to indicate statistical significance. Results: Arterial L-[2-15N]glutamine enrichments were significantly lower during enteral tracer infusion (cTTR\% IV: $6.66 \pm 0.35$ vs. EN: 3.04 $\pm 0.45 ; p<0.01$ ), reflecting first pass intestinal metabolism of L-glutamine during absorption. Compared with intravenous administration, enteral administration of L-[2-15N]glutamine resulted in a significantly higher intestinal fractional extraction of L-[2-15N]glutamine (IV: $0.04 \pm 0.07$ vs. EN: $0.31 \pm 0.11 \mu \mathrm{mol} / \mathrm{kg} / \mathrm{hr} ; \mathrm{p}=0.02$ ). Furthermore, enteral administration of L-[2-15N]glutamine resulted in higher arterial enrichments of L-[2-15N]citrulline (cTTR\% IV: $5.52 \pm 0.44$ vs. EN: $8.8 \pm 1.1 ; p=0.02$ ), and both routes of administration generated a significant enrichment $(p<0.01)$ of $L-[2-15 N]$ arginine. This was accompanied by intestinal release of $L-[2-15 \mathrm{~N}]$ citrulline across the PDV, which was highest with enteral administration of L-[2-15N]glutamine (IV:- $0.36 \pm 0.07$ vs. EN:$0.68 \pm 0.11 \mu \mathrm{mol} / \mathrm{kg} / \mathrm{hr} ; \mathrm{p}=0.03)$, and renal L-[2-15N]arginine release in both groups.

Conclusion: The gut prefers the uptake of enteral administered L-glutamine to intravenously provided L-glutamine, independent of the L-glutamine supply. Also, the route of administration, enteral or intravenous, affects the quantitative conversion of L-glutamine into L-citrulline, which is a precursor for renal L-arginine synthesis in humans.

\section{7}

\section{TREATMENT OF RECURRENT RECTAL CANCER WITH} REIRRADIATION AND SURGERY

M. Vermaas ${ }^{1}$, F.T.J. Ferenschild ${ }^{1}$, C. Verhoef ${ }^{1}$, J.J.M.E. Nuyttens ${ }^{2}$, A.M.M. Eggermont ${ }^{1}$, J.H.W. De Wilt ${ }^{1}$

1 Erasmus MC-Daniel den Hoed Cancer Center Rotterdam, Rotterdam, The Netherlands

Introduction: The standard treatment for locally recurrent rectal cancer without distant metastases is pre-operative (chemo-)radiation followed by a surgical resection with intra-operative radiotherapy on indication. Operating in a previously irradiated and surgically explored area is complicated by postradiation fibrosis and altered anatomy. Recent studies suggest a poor prognosis of previously irradiated recurrent rectal cancer. In this study we analyse the results of reirradiation and surgery for locally recurrent rectal cancer.

Patients and methods: Of a total of 117 patients with recurrent rectal cancer treated in our specialised cancer hospital, 10 patients had been irradiated previously. The median interval between primary surgery and recurrence was 24 months (range 9-117 months). After diagnosis of an isolated recurrence the patients received reirradiation with a median dose of 30Gy (range 27-40Gy) before surgery.

Results: Neo-adjuvant radiation was applied in 7 male and 3 female patients with a median age of 61 years (range 41-69 years). In all but one patient a resection of the recurrence was performed; APR $(n=3)$, resection of recurrence $(n=2)$ and total exenteration $(n=4)$; Six patients underwent a microscopically incomplete resection (R1) and 3 patients a macroscopically incomplete resection (R2), after which all patients received IORT. The minor and major complication rates were $88 \%$ and $11 \%$. There was no in hospital mortality. The overall survival at 2 and 5 years was $77 \%$ and $39 \%$, respectively. Local control after 1 and 2 years was $64 \%$ and $39 \%$. The disease free survival after 1 and 2 years was $64 \%$ and $13 \%$. Two patients presented during follow-up with complaints of severe local perineal/ pelvic pain, one with neuropathic pain in the lower extremities and four with a combination of these complaints for which all patients received continuous mediation in collaboration with an anaestesist and/or neurologist. Median pain free survival after treatment was 142 days (range 0-1654 days). The 1-year and 2 'year pain free survival percentages were $44 \%$ and $22 \%$.

Conclusion: Treatment of previously irradiated recurrent rectal cancer with reirradiation, IORT and surgery is related with high morbidity and poor local control, although some patients have long-term survival. There is a need for further research on new modalities to ameliorate the outcome of patients with locally recurrent rectal tumour. 
G.H. van Ramshorst, J. Nieuwenhuizen, P. Arends, W.C.J. Hop, J. Jeekel, J.F. Lange

Erasmus Medical Center, Rotterdam, The Netherlands

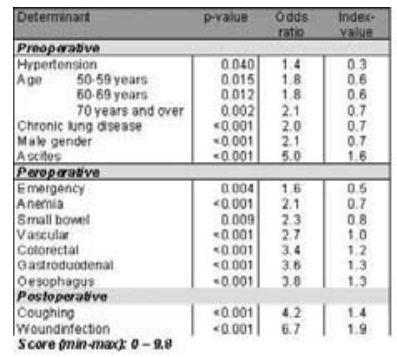

Table 1: Risk index for abdominal wound dehiscence

Introduction: Abdominal wound dehiscence is a major complication after open abdominal surgery with an incidence ranging between $0.5-3.0 \%$. Many researchers have tried to identify predisposing factors, often presenting conflicting results. In order to develop a risk index for the general surgical population, retrospective data were analyzed in this case-control study.

Methods: Medical registers from January 1, 1985, to December 31, 2005, were searched for the keywords 'dehiscence' and 'Platzbauch'. Patients who had primarily undergone appendectomy or a non-surgical (e.g. urological) operation were excluded. In total, 363 cases and 1089 randomly selected controls were included. Preoperative, intraoperative, and postoperative variables were entered in univariate analysis and multivariate stepwise logistic regression as independent predictors of wound dehiscence. Odds ratios (OR) and regression coefficients were calculated for all variables.

Results: Mean presentation of dehiscence was at 8.9 days, overall mortality was $22 \%$. Hospital stay was significantly $(p<0.001)$ longer for the dehiscence group (median 36 vs. 16 controlgroup). Recurrences developed in 29 patients ( $8.0 \%), 6$ of whom developed second recurrences. The following variables contributed significantly in multivariate analysis(OR): age (1.8 - 50-59 years, 1.8 - 60-69 years, 2.1 - 70 years and over, compared to age under 40), hypertension (1.4), chronic lung disease (2.0), male gender (2.1), ascites (5.0), emergency operation (1.6), anemia (2.1), postoperative coughing (4.2), and postoperative wound infection (6.7). Operations on the vasculatory system, esophagus, gastroduodenum, small and large bowel showed increased odds ratios. Diabetes mellitus, history of laparotomy, operating time $>2.5$ hours, malignancy, jaundice, steroid use, sepsis and vomiting were no significant predictors of wound dehiscence. Based on these findings, a risk index for the occurrence of postoperative abdominal wound dehiscence was developed. The risk index, weighing the various factors using the calculated regression coefficients, is shown in Table 1. A higher value of the score predicts a higher risk (a 1 point higher risk is associated with an Odds-Ratio of 2.7).

Conclusion: Abdominal wound dehiscence is a severe complication after open abdominal surgery. We developed a risk index, based on regression coefficients, for use in future intervention strategies.

\section{O149}

\section{ADHESION PREVENTION IN LAPAROTOMY: LONGTERM FOLLOW-} UP OF A RANDOMIZED CLINICAL TRIAL

J.B.C. van der Wal ${ }^{1}$, G.I.T. Iordens ${ }^{1}$, W.W. Vrijland ${ }^{2}$, R.N. van Veen ${ }^{1}$, J. Jeekel ${ }^{1}$, J.F. Lange ${ }^{1}$

1 Erasmus University Medical Center Rotterdam, Rotterdam,

The Netherlands

2 Sint Franciscus Gasthuis Rotterdam, Rotterdam, The Netherlands

Objective: The objective of this study was to determine the long-term effect of the use of a Hyaluronic Acid-Carboxymethylcellulose membrane (Seprafilm) on incidence of small bowel obstruction and chronic abdominal pain after colorectal surgery (Hartmann's procedure).

Background: Adhesions occur frequently after abdominal surgery and are the most common cause of bowel obstruction, chronic abdominal pain, and infertility. The risk for adhesion related readmission in the first ten years after colorectal surgery is as high as $30 \%$. To reduce the formation of adhesions, a mechanical barrier composed of hyaluronic acid and carboxymethylcellulose was developed, to prevent adherence of tissues after abdominal surgery. Short-term results of studies using Seprafilm indicate that fewer and less-severe adhesion-formation occur, however long-term results concerning the incidence of small bowel obstruction and chronic abdominal pain are lacking.

Methods: Between April 1996 and September 1998, 71 patients requiring a Hartmann procedure for sigmoid diverticulitis or obstructed rectosigmoid were randomized to either intraperitoneal placement of Seprafilm under the midline and in the pelvis or as a control.

Direct visual evaluation of the incidence and severity of adhesions was performed laparoscopically in 42 patients at second-stage surgery for restoration of the continuity of the colon. In 2006, the patients' general practitioners were interviewed by means of a questionnaire concerning their patients' health. The patients who were still alive were interviewed and asked to fill out two questionnaires concerning pain and quality of life (VAS-pain score, EQ-5D and SF-36).

Results: Of the 42 evaluated patients, 35 (16 in the Seprafilm group, 19 in the controlgroup) could be enrolled in the follow-up. Median follow-up was 105 months (range 94-119) for the Seprafilm-group and 106 (range 94-118) months for the control-group. Incidence of chronic (3 months or longer existing) abdominal complaints was significantly lower in the Seprafilm group compared to controls $(35.3 \%$ vs $77.8 \%$ respectively; $\mathrm{p}=0.018$ ). Incidence of small bowel obstruction showed a significant difference in favour of the Seprafilm group; no small bowel obstructions occurred in the Seprafilm group, whereas in the control group 5 cases of small bowel obstruction were found to have occurred $(27.8 \%, p=0.045)$. Evaluation of the quality of life questionnaires did not reveal significant differences between the two groups.

Conclusion: Seprafilm-placement provides protection against small bowel obstruction and incidence of chronic abdominal complaints.

\section{O150}

SKIN GRAFTS FOR LOWER LIMB MELANOMA RECONSTRUCTION SHOULD BE HARVESTED FROM THE IPSILATERAL SIDE

H.L. Chia ${ }^{1}$, H. Schumacher ${ }^{2}$, J. Simcock ${ }^{1}$

1 Cambridge University Hospitals Nhs Trust, Cambridge, United Kingdom

Introduction and aims: Traditional teaching has emphasised to harvest skin grafts from the contralateral side following excision of lower limb melanomas. This has been based mainly on the studies by Cade (1961) and Petersen (1962). The rationale was that tumour cells preferably spread to areas of injuries by lymphatic permeation. Flook et al (1986), however, questioned the need for this practice, suggesting that potential recurrences should be confined to the same limb by taking ipsilateral grafts. Harvest from the contralateral limb increases both the complexity of surgery and the postoperative course for the patient. This study aims to compare the cancer outcome of harvesting a skin graft from the same and contralateral limb in patients with lower limb melanomas.

Materials and methods: 85 patients with a minimum 5-year follow-up with lower limb melanoma were studied retrospectively for prognostic factors and rate and pattern of recurrence. The main outcome measures were patient demographics, site of melanoma, tumour type and AJCC stage, treatment modalities, site and side of tumour spread and survival rate. Key results: There were 22 recurrences $(25.9 \%)$, most commonly following presentation with a stage lla primary melanoma. Only 2 out of 22 recurrences were in-transit metastases. Of the patients managed with skin grafting, there was no difference in recurrence rates between a contralateral $(29 \%)$ and ipsilateral $(30 \%)$ donor site. No metastases occurred within any graft donor sites.

Conclusion: The side of skin graft harvest does not appear to influence the course of the disease. We have found no convincing evidence for the use of contralateral donor site in the literature. Given the advantages of ipsilateral donor site, we feel that patients with melanoma of the lower limb requiring a skin graft should have this harvested from the same limb.

O151

SELECTIVE MRSA SCREENING BASED ON RISK-STRATIFICATION IS INADEQUATE TO CONTROL PERI-OPERATIVE MRSA INFECTION RATES IN BREAST RECONSTRUCTIVE SURGERY

J.C.Y. Ong ${ }^{1}$, F.C. Chan ${ }^{1}$, J. Kelly ${ }^{2}$, P. Regan ${ }^{2}$

1 University College Hospital Galway, Galway, Ireland

Introduction: Screening programs based on risk stratification to control the spread of MRSA remains a major challenge for hospitals with limited 
healthcare resources. The objective of this study was to examine the efficacy of selective MRSA screening of patients who underwent postmastectomy breast reconstruction.

Methods: A retrospective, single cohort study (September 2004 to September 2006) of patients who underwent breast reconstruction surgery in a tertiary referral centre.

Results: Over the 2 year period, 70 patients underwent either immediate $(n=17)$ or delayed $(n=53)$ breast reconstruction. Utilizing risk stratification, only $24 \%$ of patients were screened preoperatively for MRSA (all negative MRSA status). A total of seven (10\%) patients developed MRSA wound infection, and six of these (86\%) patients were not screened pre-operatively. In addition, there was no statistical difference in the rate of MRSA infection in patients who underwent implant (3/7) or flap reconstruction (4/7).

Conclusion: Screening patients based on pre-operative risk stratification is insufficient as six of seven patients infected with MRSA $(86 \%)$ were considered low risk and were not screened pre-operatively. We therefore advocate MRSA screening and appropriate treatment of all pre-operative breast reconstruction patients.

\section{O152}

\section{EXPERIENCE WITH THE MENTOR CONTOUR PROFILE BECKER-35 EXPANDABLE IMPLANTS IN RECONSTRUCTIVE BREAST} SURGERY

F. Hsieh ${ }^{1}$, A. Shah ${ }^{2}$, C.M. Malata ${ }^{2}$

1 Cambridge University, Cambridge, United Kingdom

2 Plastic \& Reconstructive Surgery, Addenbrooke's Hospital Cambridge, Cambridge, United Kingdom

Introduction: For the last twenty years, single-stage expander breast reconstruction has utilized double lumen prostheses such as the round expandable implant filled with liquid silicone gel (Beckers 25 \& 50) or the anatomical expandable implant filled with firm cohesive gel (McGhan Style 150). Both these, however, have a number of drawbacks which can theoretically be circumvented by the new anatomical Becker expander filled with soft cohesive gel launched in mid-2004. The Mentor Contour Profile $₫$ Becker 35 expander was therefore selectively adopted in our practice from January 2005 in patients undergoing singlestage prosthetic breast reconstruction. A review of a single surgeon's experience with this expandable implant was undertaken to determine whether it offered any advantages over the traditional round Becker expanders or established anatomical double lumen prostheses.

Patients and methods: All patients undergoing reconstructive breast surgery using the Contour Profile $®$ Becker 35 expandable implant from January 2005 to December 2006 were retrospectively reviewed with respect to indication, implant sizes, inflation details, complications and outcomes.

Results: Over the 24 month period, 36 patients, mean age 48.9 years ( $r=14$ - 69), received 39 implants (3 bilaterally). Three quarters of the implants (29) were used for immediate breast reconstruction at the time of mastectomy. The remainder were equally divided between delayed postmastectomy reconstruction (5) and correction of congenital breast asymmetry (5). Half of the patients had simultaneous latissimus dorsi myocutaneous flap coverage of the implants. The most frequently used nominal implant sizes were $460 \mathrm{mls}$ and $565 \mathrm{mls}(r=195$ ' $565 \mathrm{mls})$. Inflation of the expanders was commenced two weeks post-operatively. An average of 3.5 inflations was needed to achieve the target expansion size and shape. Of the 25 patients with implants in situ for more than 6 months, $72 \%$ (18) have not required deflation to achieve the desired breast size and shape. The mean time from expander insertion to completion of reconstruction was 5.4 months ( $r=2$ - 9 months). Three patients required surgical intervention for a haematoma, an infected implant, and a severe capsular contracture. Additionally there were three injection port adjustments and one total capsulectomy with implant exchange giving an $18 \%$ overall revisional surgery rate (7/39 breasts). Four implants $(10 \%)$ have developed significant asymptomatic palpable rippling. The severe capsular contracture rate was $7.7 \%$ (3/39 breasts) all following postoperative radiotherapy. Most patients have, however, obtained satisfactory cosmetic results.

Conclusion: In this short term study, the Becker 35 expander was successfully used in patients undergoing breast surgery for a variety of indications with an incidence of early complications comparable to alternative prostheses. It may achieve satisfactory breast shape without the need for over-inflation. Although these preliminary data are promi- sing and the Becker 35 prosthesis has expanded the range of implants available to the breast surgeon, its exact role in reconstructive breast surgery remains to be defined.

\section{3}

LOCAL RECURRENCE OF STAGE 1 AND 2 BREAST CANCER AFTER SKIN-SPARING MASTECTOMY AND IMMEDIATE BREAST RECONSTRUCTION IN A 15-YEAR SERIES

T. Meretoja ${ }^{1}$, K. von Smitten ${ }^{2}$, M. Leidenius ${ }^{2}$, C. Svarvar ${ }^{1}$, P. Heikkil ${ }^{2}$, T. Jahkola ${ }^{2}$

1 Helsinki University Central Hospital, Helsinki, Finland
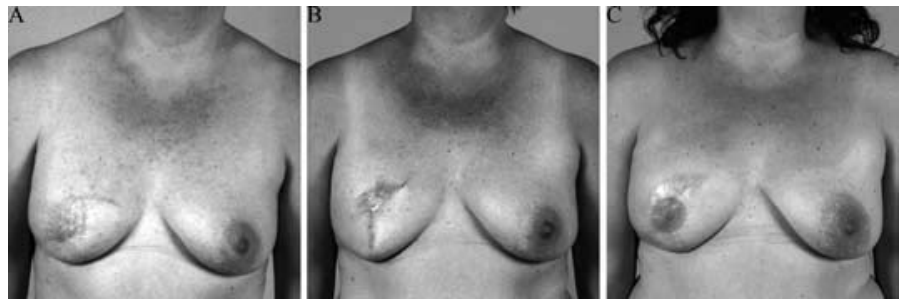

A successful SSM followed by IBR

Background and aims: Skin-sparing mastectomy facilitates optimal immediate breast reconstruction by preserving the inframammary fold and most of the breast skin. However, since the introduction of skinsparing mastectomy in 1991, concerns on local control and recurrence rates have been discussed in the literature. The aim of this study is to examine in particular incidence of local recurrence in a 15-year consecutive series of breast cancer patients having undergone skin-sparing mastectomy and immediate breast reconstruction at a single population based institution.

Patients and methods: 146 consecutive patients with either stage 1 or 2 breast cancer who underwent skin-sparing mastectomy followed by immediate breast reconstruction from 1992 to 2006 were included in this study. Retrospective analysis of the patient records was performed and follow-up data was obtained.

Results: During a mean follow-up time of 51 months, four local recurrences of the native breast skin were accounted for. In addition, three regional lymph node recurrences and four systemic recurrences took place. All of the locoregional recurrences were handled by salvage surgery followed by adjuvant oncological therapies. During a mean followup of 35 months after the detection and treatment of the locoregional recurrences none of these patients developed new recurrences. Conclusion: Our present study concludes that skin-sparing mastectomy followed by immediate breast reconstruction seems oncologically sound procedure for stage 1 and 2 breast cancer patients. In addition, our study shows that local recurrences and regional lymph node recurrences are not always associated with systemic relapse.

\section{O154}

\section{WHICH LATISSIMUS DORSI (LD) DONOR SITE SCAR SHALL I WEAR?}

A. Ismail, K. Lau, O.J. Harley, M. Ho-Asjoe

Addenbrooke's Hospital, Cambridge, United Kingdom, United Kingdom

Introduction: Latissimus dorsi myocutaneous flap was first described for breast reconstruction by Olivari in 1976 with an obliquely orientated donor site scar. Donor site concealment is an important consideration and other orientations have been subsequently described. The aim of this study was to explore current trends in women's choice of donor scar orientation and compatability with modern clothing fashions.

Methods: An anonymous questionnaire survey was conducted and the results were analysed and statistically assessed.

Results: Two hundred and five completed questionnaires of women aged between 30 and 85 were analysed. Mean age of respondent was $45(r=30-83)$. Fifty three per cent of respondents preferred the oblique scar orientation as compared to $18 \%$ who preferred the transverse scar $(p<0.05)$. This variation was seen throughout the age range but was more noticeable in the younger age group. This preference was consistent with their choice of clothing. Other scar types were also reviewed. 
Conclusion: With changes in fashion trends, patient choice of scar orientation, catering to their individual lifestyle and choice of dress, should be taken into consideration when planning LD flaps.

\section{O155}

\section{RESOURCE IMPLICATIONS OF BILATERAL BREAST RECON- STRUCTION A SINGLE CENTRES EXPERIENCE}

\section{A.J. Ponniah, J.W. Simcock, A.C. Canal, M.S. Irwin, C.M. Malata Addenbrookes Hospital, Cambridge, United Kingdom}

Introduction and aims: Since the introduction of 'Payment By Results' as part of the National Health Service (NHS) financial reforms, it has been noted by both clinicians and managers that there is an imbalance between resource use and allocated tariffs in some procedures. It is incumbent on the clinician to be aware of such discrepancies and to highlight them so that appropriate resources can be made available to maintain a high level of patient care. This study was undertaken to assess the impression that bilateral breast reconstruction was under funded.

Materials and methods: Patients who underwent bilateral breast reconstruction following mastectomy at Addenbrooke's University Hospital (Cambridge, UK) from 2000 through 2006 were identified. Resource cost analysis for each patient was based on the following parameters: number of consultants required to perform the procedure, length of procedure and hospital stay. The estimated hospital costs were then compared to the national tariff for the Healthcare Resource Group (HRG) 'Complex Breast Reconstruction using Flaps'.

Key results: Over the 7 year period 40 patients underwent bilateral breast reconstruction. Of these, 15 had implant only and 25 had flap reconstructions. Of the flap reconstructions 8 patients had latissimus dorsi pedicled flaps and 17 had abdominal pedicled or free flaps. The mean operative time was $9.5 \mathrm{hrs}(£ 4.5 / \mathrm{min}$ ) and mean hospital stay was 10 days (£150/day). Based on 2 reconstructive consultant surgeons (£34/hr/consultant) operating each episode costs $£ 4711$. The national tariff for this procedure is $£ 4053$.

Discussion: Cost analysis is a complex process with many facets. The government's allocated tariff appears low even before hidden costs are included. These hidden costs may contribute to the hospital deficits which in turn threaten the level and quality of patient care. Problems highlighted by this study include inaccurate coding and a lack of appropriate codes. The financial reforms are evolving yearly following feedback from clinicians and managers. There are new HRGs proposed for breast reconstructions, however they do not address the costs of bilateral breast reconstructions. With increasing financial pressure on NHS Trusts there is danger of a drive toward simpler operations which receive proportionally greater remuneration.

\section{O156}

CONSEQUENCES OF SURGICAL TRAUMA ON DERMAL WOUND

HEALING: COMPREHENSIVE ANALYSIS OF THE SKIN REGENERATION AFTER PARTIAL HEPATECTOMY

\section{H. Sorg, T. Schulz, B. Vollmar}

Institute for Experimental Surgery, Rostock, Germany

Introduction: The skin is the biggest organ of the human being. Therefore the management of wounds is of special interest in medicine but especially in surgical disciplines. Skin regeneration itself represents a complex biological process that requires distinct cellular interactions among different types of cells. Several factors like metabolic disorders, cachexy and infections may affect the physiological skin restoration with the consequence of insufficient healing and/or deforming scar formation Herein, we investigated the significance of surgical trauma on dermal wound healing in an established animal model of major abdominal surgery.

Materials and methods: Within the ears of hairless SKH1-mice $(n=23$; ip anaesthesia ketamine/xylazine $(90 / 25 \mathrm{mg} / \mathrm{kg} \mathrm{bw})$, skin and subcutaneous tissue was excised down to the cartilage (depth $\sim 150 \mu \mathrm{m}$ ), thus creating a full dermal thickness wound of $\sim 2.4 \mathrm{~mm} 2$. In this model of physiologic wound healing, we analyzed by means of intravital fluorescence microscopy the kinetics of wound healing at days 3, 5, 7 and 10 post surgery with assessment of wound epithelialization and nutritive perfusion/angiogenesis at the margin as well as distal of the wound (FCD, functional capillary density). To simulate major surgical trauma, a $70 \%$ partial hepatectomy was performed in eight animals ( $\mathrm{pHx} ; \mathrm{n}=8$ ), while six animals underwent only sham-operation, i.e. laparotomy with manipulation of the liver, but without resection (sham; $n=6$ ). Animals without abdominal surgery served as controls for physiological wound healing (control; $n=9$ ). Morphological characterization of the regenerating skin was performed by histology (H\&E-staining) and quantitative immunohistochemistry of tissue specimens (PCNA, proliferating cell nuclear antigen). Data are given as mean \pm SEM; ANOVA and post-hoc comparison.

Results: Wound closure of $\mathrm{pHx}$-animals was found markedly deteriorated when compared to controls, as indicated by the significantly lower values of epithelialization over the whole observation period of ten days after surgery (day 3: $29 \pm 2 \%$, day $5: 53 \pm 3 \%$, day $7: 72 \pm 2 \%$ and day 10 : $89 \pm 3 \%$, p $<0.05$ each vs controls: $40 \pm 2 \%, 74 \pm 4 \%, 91 \pm 2 \%$, and $100 \pm 0 \%$ ) Concomitantly, FCD serving as a parameter of nutrition and neovascularization during wound healing was significantly reduced at day 7 and day 10 upon $\mathrm{pHx}$. Impairment of skin regeneration was further confirmed by PCNA-immunohistochemistry, demonstrating lower numbers of proliferating cells within the wound. Of utmost interest, laparotomy with manipulation of the liver was also associated with an almost comparable extent of deterioration in wound healing in the sham-operated animals at early time points after surgical intervention (epithelialization: day 3 : $34 \pm 2 \%$ and day 5: $60 \pm 2 \%$ ). However, this difference in wound healing between sham and control animals could not be observed at later time points, implying a minor extent of surgical stress.

Conclusion: Our study impressively demonstrates the pivotal role of surgical trauma and trauma-associated paracrine effects in regenerative processes, such as skin healing. Therefore, much effort should be paid on the development of strategies which allow limiting surgical traumaassociated perturbations of regeneration.

\section{7}

DEPOSITION AND PRODUCTION OF THE COMPLEMENT PROTEIN MANNOSE-BINDING LECTIN (MBL) IN ABDOMINAL AORTIC ANEU. RYSM (AAA); A POSSIBLE ROLE IN THE DEVELOPMENT OF AAA

R. A. Matthijsen, F.A.M.V.I Hellenthal, S Heeneman, N.T. Hoebers, W.A. Buurman, G.W.H. Schurink

University Hospital Maastricht, Maastricht, The Netherlands

Inflammatory mechanisms are at the basis of abdominal aortic aneurysm (AAA) development and progression. Involvement of complement, as an important mediator of the innate immune system, is thereby still unknown. The complement system and in particular MBL enables an organism to recognize different exogenous as well as endogenous danger signals. Activation of the complement system and more specifically MBL in AAA can be assumed due to the presence of several endogenous danger signals. Frozen biopsies from elective and emergency surgical aortic reconstructions for AAA were selected from our institutional database. Biopsies were immunohistochemically studied for CD68 positive macrophages and complement factors including MBL, C1q and C3. These results are the first to show the presence of the complement protein MBL in AAA. MBL primarily co-localized with CD68 positive inflammatory cells, which were characterized as infiltrated macrophages. Other complement factors, such as C1q and C3 were also detected. Both of which showed limited co-localisation with MBL. Investigation of the origin of MBL in AAA by means of polymerase chain reaction (PCR) technique showed the endogenous production of MBL in AAA tissue. The co-localisation of MBL in and around macrophages might implicate that macrophages are responsible for MBL synthesis in AAA. In order to test this hypothesis we studied the capability of macrophages and undifferentiated monocytes to produce MBL in vitro. Both cell types proved to be able to produce MBL.

Conclusion: 'These results show for the first time the activation and deposition of complement factors in AAA. Of particular interest in this process is the binding of MBL, primarely around macrophages in the aneurysmal wall. Moreover, the data show for the first time the endogenous synthesis of MBL in the aneurysmal wall of AAA. Our in vitro results demonstrate that the human monocyte and macrophage have the ability to synthesize MBL and are most probably responsible for the observed $M B L$ production in the AAA. These inflammatory cells might therefore be an important extra hepatic source of MBL at sites of inflammation in the aneurysmal wall. The extensive deposition and production of MBL in AAA tissue is suggestive of a prominent role for MBL in AAA pathogenesis. 
O158

EARLY RAISE IN SERUM VASCULAR ENDOTHELIAL GROWTH FACTOR (VEGF) AND PLATELET DERIVED GROWTH FACTOR (PDGF) PREDISPOSES FOR RESTENOSIS AFTER EVERSION ENDARTERECTOMY IN PATIENTS WITH A NORMAL MANNOSEBINDING LECTIN GENOTYPE

K.H. Hirschberg1', A. Szab², E. Dosa², Z. Prohszka², L. Entz², G. Füst², L. Cervenak ${ }^{2}$

1 Semme/weis University, Budapest, Hungary

Introduction: Experimental and clinical evidences suggest that myointimal proliferation following carotid endarterectomy may be responsible for early restenosis. Vascular endothelial growth factor (VEGF) and platelet derived growth factor (PDGF) have been suggested to play a role in this process. Recently we found that the incidence of restenosis during a follow up period of 14 months was significantly higher in patients homozygous for the normal genotype of mannose-binding lectin (MBL) as compared to patients who carried MBL variant alleles. Therefore, we investigated a possible correlation between restenosis and the preoperative levels or early postoperative changes of the serum VEGF and PDGF concentrations and hypothesized that a possible relationship to the MBL system may exist.

Methods: The study was performed in 82 patients who underwent carotid eversion endarterectomy, and were followed-up by carotid duplex scan (CDS) for 14 months. VEGF and PDGF levels were measured by enzime-linked immunosorbent assay (ELISA) in samples obtained preoperatively and 4 days postsurgery. MBL genotypes were analyzed by a PCR (polimerase chain reaction)-based method.

Results: Pronounced (in the highest tertile) increase of both VEGF and PDGF predicted restenosis in patients who were homozygous for the normal MBL genotype. In this group the odds ratio of restenosis in patients with high versus low early VEGF increase was 16,7 and 20,0 for the 7 months and 14 months CDS values, respectively. Similar $(5,2$ and 7,0 ) albeit less significant association was found with early PDGF increment as well.

Conclusion: These findings indicate that development of restenosis depends both on complement activation regulated by a genetic factor and on the intensity of probably hypoxia-induced pathological processes leading to enhanced production of VEGF and PDGF during the very early postoperative period.

\section{O159}

TUMOR VASCULAR MANIPULATION WITH TNF MEDIATES A MORE HOMOGENOUS DISTRIBUTION OF LIPOSOMES IN MURINE MELANOMA THAT CONTRIBUTES TO A BETTER TUMOR RESPONSE

A. Seynhaeve, S. Hoving, D. Schipper, C. Vermeulen, G. aan de WielAmbagtsheer, S. van Tiel, A.M.M. Eggermont, T. ten Hagen Erasmus Medical Center Rotterdam, Rotterdam, The Netherlands

Introduction: Adequate drug uptake at the tumor site remains a crucial problem in solid tumor therapy. Recently, tumor vascular normalization has been proposed to enhance drug delivery and improve tumor response to chemotherapy. Differently however, augmenting leakage of the tumor-associated vasculature with the use of tumor necrosis factoralpha (TNF), and as such enhance tumor vascular abnormality, may improve intra-tumoral drug concentrations and tumor response as well. Materials and methods: To investigate a better drug delivery we used gallium or fluorescent labeled liposomes in combination with TNF in $\mathrm{B} 16 \mathrm{BI} 6$ melanoma-bearing mice. We studied the effect of TNF on intra-tumoral distribution of liposomes with the use of the dorsal skin fold-chamber and intra-vital microscopy. Results. Addition of TNF to systemic injection of $100 \mathrm{~nm}$ liposomes, improved tumor accumulation by 5 to 6 -fold. Accumulation of 400 and $800 \mathrm{~nm}$ liposomes in tumor was much less pronounced. Intra-vital microscopy showed that only 100 $\mathrm{nm}$ liposomes extravazated effectively in the presence of TNF, while liposomes of $400 \mathrm{~nm}$ and larger remained in general inside the blood vessels and minimal leakage in the surrounding tissue was observed even in the presence of TNF. Next to that, we observed in vivo that the tumor cells take up the liposomes intact followed by intracellular breakdown. Discussion. Our results indicate that more tumor vessels become permeable by TNF, leading to a more homogeneous distribution of the liposomes throughout the tumor, which is crucial for an optimal tumor response. We conclude that delivery of nano-particulate drug formulations to solid tumor benefits from augmenting the vascular leakage through vascular manipulation.
0160

INTRAMUSCULAR AND COMBINED INTRAMUSCULAR/INTRA-ARTERIAL ADMINISTRATION OF BONE MARROW MONONUCLEAR CELLS IN PATIENTS WITH ADVANCED LIMB ISCHEMIA

R.B.M. van Tongeren, J.F. Hamming, W.E. Fibbe, J.H. van Bockel, J.H. Lindeman

University Medical Center Leiden, Leiden, The Netherlands

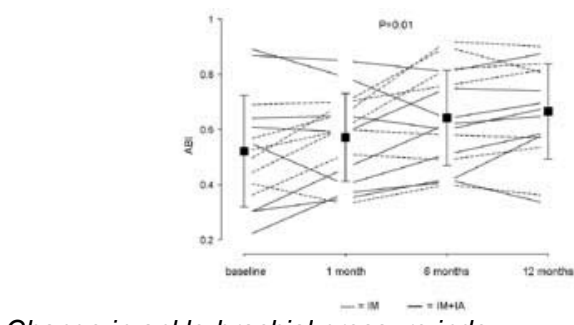

Change in ankle-brachial pressure index

Background: A substantial number of patients with severe peripheral arterial occlusive disease (PAOD) is left without options for surgical or endovascular treatment. Recent evidence suggests that bone marrow mononuclear cells (BMC) may promote collateral vessel formation in these patients. However, several critical aspects such as long-term safety, effect durability, and optimal administration mode require consideration. We evaluated feasibility and safety of exclusively intramuscular versus combined intramuscular/intra-arterial delivery of BMC in patients with severe PAOD who were no candidates for surgical or endovascular treatment.

Methods and results: 27 patients were treated with either combined intramuscular/intra-arterial $(n=12)$ or sole intramuscular delivery $(n=15)$ of autologous BMC. Efficacy was assessed after 1, 6 and 12 months by clinical and angiographical parameters. There were no complications directly related to injection of the cells. Two patients suffered from post-operative cardiac failure. Nine patients required limb amputation because of ongoing critical ischemia. Three patients died within the first year of follow-up due to non-procedure related causes (2 after earlier amputation). BMC treatment in the remaining group resulted in a significant and sustained (>12 months) improvement. Mean ankle-brachial pressure index $(A B I)$ increased $23 \%$ after 6 months $(p=0.01)$. Pain-free walking distance improved from $81 \pm 56$ meters at baseline to $257 \pm 126$ meters at $t=6$ months $(p=0.0002)$ and painscore reduced for up to $50 \%$ as shown by Brief Pain Inventory $(p=0.001)$. Evaluation of angiographies showed remarkable diversity in collateral vessel development. We observed a trend favouring combined delivery $(p=0.17)$.

Conclusion: Both intramuscular and combined intramuscular/intraarterial delivery of autologous BMC are safe, and result in persistent improvement in a considerable proportion of patients with severe PAOD who are not amendable for conventional treatment.

\section{O161}

ENDOTHELIAL NITRIC OXIDE SYNTHASE ACTIVITY IS ESSENTIAL FOR VASODILATION DURING BLOOD FLOW RECOVERY, BUT NOT FOR ARTERIOGENESIS

B.M.E. Mees ${ }^{1}$, S. Wagner ${ }^{2}$, E. Ninci², S. Tribulova² ${ }^{2}$ R. van Haperen', M. Heil' ${ }^{2}$ R. De Crom ${ }^{1}$, W. Schaper

1 Erasmus University Medical Center Rotterdam, Rotterdam,

The Netherlands

2 Max-Planck Institute, Bad Nauheim, Germany

Introduction: Arteriogenesis (collateral artery growth) is the major mechanism of vascular growth, which is able to compensate for blood flow deficiency after arterial occlusion, and therefore the most promising for therapeutic neovascularization. Endothelial nitric oxide synthase (eNOS) activity is essential for neovascularization. However, the role of eNOS in arteriogenesis remains unclear due to the absence of in vivo models for this specific process of vascular growth only.

Methods: We analyzed blood flow recovery and collateral artery growth in an arteriogenesis-specific hind limb model, comparing both transgenic eNOS overexpressing (eNOStg, $\mathrm{n}=21$ ) and eNOS deficient (eNOS-/-, $n=26$ ) mice with wild type (WT, $n=23$ ) controls.

Results: Tissue perfusion in the feet, measured by Laser Doppler imaging (LDI), and collateral-dependent blood flow in the calf, measured by magnetic resonance imaging (MRI), were significantly increased 
in eNOStg mice only immediately following ligation (LDI: $33 \pm 4 \%$ vs. $18 \pm 4 \%, \mathrm{P}<0.01$; eNOStg vs. WT) suggesting higher acute maximal vasodilation in eNOStg mice. In eNOS-/- mice tissue perfusion remained decreased until endpoint (21 day: $37 \pm 4 \%$ vs. $85 \pm 4 \%, \mathrm{P}<0.001$; eNOS/- vs. WT). However, MRI measurements in eNOS-/- mice demonstrated diminished collateral-dependent blood flow only in the 1 st week after ligation ( 3 day: $40 \pm 7 \%$ vs. $12 \pm 2 \%, P<0.001 ; 14$ day: $89 \pm 13 \%$ vs. $83 \pm 8 \%$, $\mathrm{P}=\mathrm{NS}$; WT vs. eNOS-/-), suggesting normal, perhaps delayed collateral growth. Treatment with the NO-donor SNAP at day 7 resulted in an acute increase in collateral blood flow via vasodilation in eNOS-/- mice, while in WT mice no increase in collateral blood flow was seen, as collateral arteries in WT mice were already maximally vasodilated. Finally, extensive histology confirmed no differences in diameter, wall area or wall thickness between collateral arteries of eNOStg, eNOS-/- and WT mice both at day 7 and 21 .

Conclusion: In summary, our results show no beneficial effects of eNOS overexpression on arteriogenesis. Furthermore, we evidence that impaired blood flow recovery in eNOS-/- mice was not caused by decreased collateral artery growth, but by insufficient nitric oxide (NO)mediated vasodilation of collateral vessels. These findings stress the crucial role of NO-mediated vasodilation in the early phase of blood flow recovery after arterial occlusion and present an additional mechanism in the process of (therapeutic) neovascularization.

\section{O162}

\section{MRI OF INFLAMMATORY CELLS IN ABDOMINAL AORTIC ANEURYSMS: TOWARDS WALL STRENGTH IMAGING}

M. Truijers, J.J. Fütterer, S. Takahashi, J.D. Blankensteijn, J.O. Barentsz UMC St Radboud Nijmegen, Nijmegen, The Netherlands

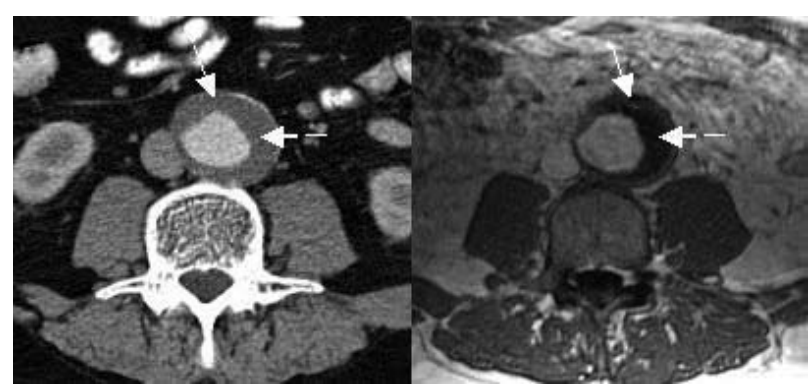

Clear signal loss (blackening) in the wall of $A A A$

Background: Aneurysm formation and aortic wall weakening are associated with extensive inflammatory cell recruitment, particularly involving macrophages. The non-invasive detection of infiltrated macrophages could therefore provide information on the degree of inflammation (wall weakening), the efficacy of anti-inflammatory medical treatment, and aneurysm rupture risk.

Objective: To investigate the uptake of a macrophage specific contrast agent (ultrasmall particles of iron oxide or USPIO) in the aneurysm and non-dilated aortic wall using Magnetic Resonance Imaging.

Methods: 11 patients with an aortic or iliac aneurysm $(\geq 3.0 \mathrm{~cm}$ or $\geq 2.0$ $\mathrm{cm}$, respectively) and 11 age matched controls were selected after reviewing 239 USPIO enhanced abdominal MRI scans. USPIO uptake was assessed by counting the number of quadrants showing clear subendothelial signal voids. Signal loss caused by calcifications and intramural haematomas were identified after fusing MR and CT data. Results: Image fusion, based upon vascular landmarks like the aortic bifurcation, resulted in adequate alignment of CT and MR data and the identification of focal signal voids caused by USPIO uptake. Although clear foci of signal loss were seen in both the aneurysm and normal aortic wall, more positive quadrants (signal loss) were found in the aneurysm group $(21.1 \pm 9.6$ vs. $3.3 \pm 2.2$ respectively, $p=0.03)$. Conclusion: MR imaging of aortic wall inflammation is a promising technique with the potential of providing valuable information on aneurysm wall strength, aneurysm rupture risk and the efficacy of future anti-inflammatory medical treatment of abdominal aortic aneurysms (e.g. statin, doxycycline). Studies have been intiated to correlate USPIO uptake with aneurysm wall strength.
O163

OVEREXPRESSION OF ENDOTHELIAL NITRIC OXIDE SYNTHASE RESTORES POST-NATAL NEOVASCULARIZATION IN ATHEROS-

\section{CLEROSIS}

B.M.E. Mees ${ }^{1}$, L. Waeckel ${ }^{2}$, T. Ebrahimian², D. You², D. Tempel ${ }^{1}$, J. Vilar ${ }^{2}$, R. van Haperen ${ }^{1}$, B. Levy², R. De Crom¹, J.S. Silvestre ${ }^{2}$ 1 Erasmus University Medical Center Rotterdam, Rotterdam,

The Netherlands

2 Inserm U689, Paris, France

Introduction: Alteration in post-ischemic neovascularization is a common complication of atherosclerotic disease. This results, at least in part, from abrogation of bone-marrow mononuclear cells (BM-MNC) pro-angiogenic potential. Overexpression of eNOS has been shown to promote vessel growth in the setting of ischemia. We therefore hypothesized that elevation of eNOS expression can restore the impaired postnatal vasculogenesis and neovascularization in atherosclerotic (ApoE KO) mice.

Methods: Hind limb ischemia was induced in the mice by right femoral artery ligation. Two weeks after ligation we evaluated tissue perfusion of the foot by Laser Doppler Imaging (LDI) and vessel density in the hind limb by micro-angiography (arterioles) and histology (capillaries). In vitro BM-MNC apoptosis, -proliferation and -differentiation assays were performed.

Results: In a first set of experiments, tissue perfusion and vessel density were 1.5-fold increased in transgenic mice overexpressing human eNOS (eNOStg) as compared to wild type C57BI6 (WT) mice $(\mathrm{P}<0.001, \mathrm{n}=10)$. Furthermore, transplantation of $1 \times 106$ WT BM-MNC or eNOStg BM-MNC in WT recipients $24 \mathrm{hrs}$ after ligation both caused a 1.5-fold increase in tissue perfusion and vessel density compared to injection of $\mathrm{PBS}(\mathrm{P}<0.001, \mathrm{n}=10)$. In a second set of experiments we used ApoE KO mice and crossbreeds of eNOStg and ApoE KO mice (eNOStg*ApoE KO). Both tissue perfusion and vessel density were 1.8-fold increased in eNOStg*ApoE KO mice as compared to ApoE KO mice $(P<0.001, n=10)$. In addition, transplantation of eNOStg ${ }^{*} A p o E K O$ $\mathrm{BM}-\mathrm{MNC}$ in ApoE KO recipients caused a 1.6-fold increase in tissue perfusion and vessel density compared to PBS $(P<0.01, n=10)$, while transplantation of ApoE KO BM-MNC had no positive effect on tissue perfusion and vessel density compared to PBS. eNOS overexpression did not affect BM-MNC apoptosis but increased their ability to differentiate in vitro into endothelial progenitor cells.

Conclusion: The above findings demonstrate that eNOS overexpression in the endothelium improves post-ischemic neovascularization in both physiological (WT) as atherosclerotic (ApoE KO) settings. Furthermore, eNOS overexpression in the bone marrow restores the impaired pro-angiogenic potential of atherosclerotic bone marrow. Therefore, overexpression of eNOS could play a vital part in the development of therapeutic angiogenesis for atherosclerotic disease.

\section{O164}

\section{SUPERVISED EXERCISE THERAPY FOR INTERMITTENT CLAUDICATION IN A COMMUNITY-BASED SETTING}

S.P.A. Nicolai, B.L.W. Bendermacher, L.M. Kruidenier, A.A.H.R. Dahlmans, J.P. Houtermans, E. Eussen, R.J.Th.J. Welten, J.A.W. Teijink Atrium Medical Center Parkstad, Heerlen, The Netherlands,

Introduction: Exercise therapy is highly efficacious for symptomatic relief in peripheral arterial disease (PAD). A recent Cochrane review identified a significant improvement in walking distance in patients undergoing a supervised exercise therapy (SET) program compared with those involved in a non-supervised program. Results in the literature are based on clinic-based SET. Disadvantages of clinic-based SET are limited capacity, transport costs and transport is time consuming. To overcome these problems, a community-based concept of SET was developed. The Network for Exercise Therapy, Parkstad (NETP) consists of 29 physiotherapeutic practices equally dispersed over the south-eastern part of Limburg.

Objective: The objective of this study was to determine the effect of SET in a community-based setting.

Patients and methods: All consecutive patients with intermittent claudication who had no previous (vascular) intervention for PAD, were considered for community-based SET. SET was administered according to the guidelines of the Royal Dutch Society for Physiotherapy. At baseline and at 1,3 , and 6 months, the initial claudication distance (ICD) and the 
absolute claudication distance (ACD) were measured by a progressive treadmill exercise test. For each patient the change in ICD and ACD expressed as the percentage increase in walking distance compared with baseline, was calculated.

Results: From January through October 2005, 93 consecutive claudicants were eligible. Overall, 37 patients discontinued the SET program. Eleven of these patients stopped because of intercurrent disease, while for 10 SET did not lead to adequate improvement and underwent a vascular intervention. Three patients quit the program stating that they were satisfied with the regained walking distance. Ten patients were not motivated sufficiently to continue the program, and in three patients, a lack of adequate insurance coverage was the reason for dropping out. Data for 56 patients were used, showing a mean percentage increase in ICD of $187 \%$ and $240 \%$ after 3 and 6 months, respectively. Mean percentage of the ACD increased $142 \%$ and $191 \%$ after 3 and 6 months, respectively.

Conclusion: Supervised exercise therapy in a community-based setting seems a promising approach to providing conservative treatment for patients with intermittent claudication.

\section{O165}

RECTAL CANCER: RESULTS FROM A GENERAL TEACHING HOSPITAL COMPARED TO AN UNIVERSITY HOSPITAL TEN YEARS AFTER THE TME-TRIAL

\section{J.G. Bloemen ${ }^{1}$, S.E.M. Engelen², G.L. Beets ${ }^{2}$, J.L.M Konsten ${ }^{1}$ \\ 1 Viecuri Hospital Venlo, The Netherlands,}

2 Academic Hospital Maastricht, Maastricht, The Netherlands

Introduction: The local recurrence rate after rectal cancer surgery dropped dramatically after the introduction of pre-operative radiotherapy and TME surgery. However, complications such as anastomotic leakage, pneumonia, sexual disorders, perineal wounddehiscence and fecal incontintince are common. These findings can influence quality of life. The purpose of this study was to evaluate the difference in morbidity, mortality, survival and quality of life after surgery for rectal cancer, comparing an Unversity Hospital (UH) and a General Teaching Hospital (GTH), with the results of the dutch TME trial as a standard.

Methods: All patients with a resectable adenocarcinoma of the rectum operated upon between 2003 and 2005 were retrospectively included in this study. Data about preoperative staging, surgical treatment and complications were collected. Quality of life was assessed with the EORCT QLQ CR 30-38 questionnaire and the IQOAL-SF38 questionnaire was used to assess the impact of fecal incontinence.

Results: 229 patients were included (GTH 130, UH 99). The average age was 69 years in GTH (range 34-98) and in UH 71 years (range 41-90). T3 and T4 tumours were significantly more found in the UH $(80,8 \%$ vs. $65,3 \%)$. In the UH more neoadjuvant chemoradiation was given $(32 \%$ vs. $4,9 \%)$ whereas in GTH $5 \times 5$ gray was standard neoadjuvant treatment ( $87 \%$ vs $54 \%) .53,2 \%$ of the patients got a low anterior resection with a protective ileostomy in the $\mathrm{GH}$ while $35,6 \%$ got a low anterior resection with a protective colostomy in the $\mathrm{UH}$. In UH more APRs were done $(25,4 \%$ vs. $16,9 \%)$, whereas there was no difference in the localization of the tumour. The admission duration was on average 15,4 days in GTH and 23,0 days in $\mathrm{UH}(p<0,01)$. In UC $63,6 \%$ of the patients had a complication; $20,4 \%$ anastomotic leakage, $17,3 \%$ woundinfections, $7,2 \%$ pneumonia. The mortality was $7,1 \%$. In GTH $58,5 \%$ had a complication; $14,6 \%$ anastomotic leakage, $9,2 \%$ pneumonia, $3 \%$ woundinfection. The mortality was $4,6 \%$. Results of resections were in GTH: CRM < $1 \mathrm{~mm} \mathrm{9.2 \% ,} \mathrm{CRM} \mathrm{>} 1 \mathrm{~mm} \mathrm{90.8 \% .} \mathrm{UH:} \mathrm{CRM} \mathrm{<} 1$ $\mathrm{mm} 14.1 \%$, CRM $>1 \mathrm{~mm} 85,9 \%$. Overall 3 -year survival is $78,0 \%$ in GTH and $74,4 \%$ in UH $(p>0,05)$. Results regarding quality of life will be presented on the congress.

Conclusion: In GTH the morbidity and mortality is lower than in $\mathrm{UH}$, with more radical resections. However, this doesnot result in a better survival. The results from both hospitals are not as good as the results from the TME trial, with a morbidity and mortality of respectively $47 \%$ and $3 \%$.

\section{6}

A NEW METHOD FOR BILIARY DRAINAGE; CONTROLLED EXPERIMENTAL STUDY IN IATROGENIC OBSTRUCTIVE JAUNDICE DOGS

A.H.I. Helmy ${ }^{1}$, M. Esawi ${ }^{1}$, H. Rizk ${ }^{1}$, H. Ezzat ${ }^{1}$, O.M. Hammam¹, A.F. El Kased ${ }^{2}$
1 Theodoer Bilharze Research Institute, Caïro, Egypt 2 Faculty of Medicine Meofeya University, Menofeya, Egypt

Objective: latrogenic Bile duct injury is a major complication after cholecystectomy and other types of upper abdominal operations. The standard treatment is Roux-en-Y hepatico-jejunostomy and other nonsurgical treatment. We used polytetrafluoroethylene (PTFE) grafts as an option for repair of bile duct injury and biliary drainage.

Materials and methods: 15 dogs were used in this study. In the 1 st laparotomy; iatrogenic obstructive jaundice by tying the common bile duct, 7 days later the 2nd laparotomy; biliary drainage doing hepateciodudenostomy using the (PTFE) grafts reinforced and non-reinforced. The 3rd laparotomy; obtain samples liver bx, the two types of grafts and serum samples of bilirubin and alkaline phosphatase.

Results: There was no mortality in this study. 6,6 and 3 dogs were sacrificed after 1,3 and $>6$ months respectively. All animals had no leakage, fistulae, bleeding and peritonitis. Jaundice was relived after the 2nd laparotomy detected by the stool colour, weight gain, serum bilirubin and alkaline phosphatase. The main complications were wound infection and burst abdomen which was repaired in the 2nd laparotomy. Dogs start to gain weight after 2 weeks of 2 nd laparotomy. Bil and Alk phos returned to normal after 2-4 weeks from the 2nd laparotomy in reinforced and non-reinforced grafts respectively. Histologicaly the liver architect showed relief of the obstructive jaundice and sealed fibrosis around and from within the two types of grafts in all intervals.

Conclusion: The use of reinforced (PTFE) graft to correct iatrogenic obstructive jaundice is an efficient, effective technique and the reinforced is doing better and could be used clinically as an alternative to other procedures.

\section{O167 \\ INFLUENCE OF DIFFERENT COATINGS IN ADHESION FORMATION WITH PROSTHETIC MESHES}

M.H.F. Schreinemacher ${ }^{1}$, P.J. Emans ${ }^{1}$, M.J. Gijbels², L.H. Koole ${ }^{2}$, G.L. Beets ${ }^{1}$, N.D. Bouvy ${ }^{1}$

Academic Hospital Maastricht, Maastricht, The Netherlands

Background: Incisional hernias occur frequently (10-30\%) and the best method of repair is the use of a prosthetic mesh. Although an inflammatory response is needed for incorporation of the mesh with the abdominal wall, a frequent side effect is adhesion formation. These adhesions can give significant complaints. In this rat study we compared a non-coated polypropylene (PP) mesh to five differently coated meshes in regard to adhesion formation

Methods: 63 rats were divided into six groups, each receiving a diffe-

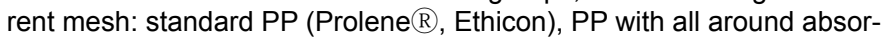
bable coating (Ultrapro $(\mathrm{R}$ and Proceed $\mathrm{R}$, Ethicon), PP with all around titanium coating (TiMesh Light $(\mathrm{R}, \mathrm{GfE})$, polyester with a one sided absorbable layer of collagen (Parietex composite $\mathrm{R}$, Sofradim) and a PP mesh coated all around with a NVP/BMA copolymer developed at the department of Biomaterials, Maastricht University. All meshes were placed intra-abdominally and fixated with two polyglactin sutures $($ Vicryl $\mathbb{R}$, Ethicon). At one and four weeks follow up (FU), the rats were sacrificed. Host response was measured macroscopically for extent, type and tenacity of adhesions, constituting total adhesion score. Incorporation with the abdominal wall was also assessed. The specimen were prepared for histology and scored for inflammatory cells and adhesions. All groups were compared to Prolene. Statistics were performed by Mann-Whitney and Fisher's exact test. P-values $<0,05$ were considered significant. Results: Two rats died directly postoperatively, the others recovered well without adverse effects. All 61 animals showed adhesions to omentum and/or scrotal fat. Thirty animals also had adhesions to liver (45\%), spleen $(28 \%)$ and bowel $(23 \%)$. At one week FU total adhesion score for Ultrapro, Proceed, TiMesh, and NVP/BMA coated PP was significantly lower compared to Prolene $(p<0,005)$. With Ultrapro the number of animals with adhesions was also significantly lower (0 VS $67 \%$, p0,045). Incorporation was often observed only at the surrounding area of the two sutures and no significant differences were found between all groups. Especially fibroblasts, macrophages were seen more often in the Proceed and Ultrapro group, indicating a more active inflammatory reaction. At four weeks FU, Parietex and NVP/BMA coated PP still had significantly better total adhesion scores compared to Prolene $(p<0,05)$. Although not significant, both groups also showed no adhesions to organs, compared to $29 \%$ adhesions in the Prolene group. Ultrapro sho- 
wed more adhesions at four weeks then at one week FU $(83 \%, \mathrm{p} 0,078)$. Similar to one week FU, incorporation remained poor for all groups. More macrophages were observed in the Proceed and Ultrapro group with the absorbable layer of Proceed less fagocytosed, but with more fibroblasts present. Total adhesion score of Proceed was significantly worse at four weeks compared to one week FU $(\mathrm{p} 0,04)$.

Conclustion: The ideal mesh provides good incorporation with the abdominal wall and yet no adhesions to the visceral side. Different coatings have been developed to minimize these adhesions. Although they show a better outcome at one week, effects do not seem to last with Ultrapro, Proceed and Timesh. Parietex and a new NVP/BMA coating on PP do seem to improve adhesion outcome.

\section{8}

HELICOBACTER GENOTYPING IN PATIENTS WITH PERFORATED PEPTIC ULCER

N. A. P. Komen ${ }^{1}$, M. J. O. Bertleff², L. J. van Doorn³, J. F. Lange', P. W. de Graaf ${ }^{4}$

1 Erasmus Medical Center Rotterdam, Rotterdam, The Netherlands 2 Diaconessenhuis, Leiden, The Netherlands

3 DDL Diagnostic Laboratory, Voorburg, The Netherlands

4 Reinier de Graaf Groep, Delft, The Netherlands

Introduction: Helicobacter pylori plays an important role in the pathogenesis of peptic ulcer disease. Several genotypes have been associated with different clinical entities like gastritis and peptic ulcer. However, to date, little is known about the possible association between the $\mathrm{H}$. pylori genotype and perforated peptic ulcer (PPU). Finding a specific Helicobacter genotype associated with PPU, would allow screening and prevention by means of prophylactic eradication therapy. The primary aim of this study was to evaluate which genotypes are present in patients with PPU and which genotype is dominant in this population. The secondary aim was to study the possibility of determining the $\mathrm{H}$. pylori status in a way other than by biopsy.

Methods: Serum samples, gastric tissue biopsies, lavage fluid and fluid from the naso-gastric tube were collected from patients operated upon for PPU. The materials were frozen immediately after collection. By means of PCR, DEIA and LIPA the presence of the 'cytotoxin associated gene' (cagA) and the genotype of the 'vacuolating cytotoxin gene' was determined.

Results: A total of 30 patients were included, of which 9 were women. The average age was 65 years. Ten patients were operated laparoscopically. A prepyloric perforation was found in 11 patients, a pyloric perforation in 8 and 11 patients had a postpyloric perforation. Fluid from the naso-gastric tube was obtained from 25 patients, lavage fluid from 26 patients, serum samples from 20 patients and biopsies from 18 patients. In these materials a diversity of genotypes was found, of which no specific cagA/vacA genotype appeared to be dominant. However, a correlation was found between the $\mathrm{H}$. pylori presence in biopsy and its presence in lavage fluid $(p=0,001)$.

Conclusion: This study shows many different genotypes can be present in patients with PPU, however no genotype was found to be dominant. The correlation between $\mathrm{H}$. pylori presence in biopsy and its presence in lavage fluid suggests that intraoperative biopsy is unnecessary and that analysis of lavage fluid is sufficient to determine $\mathrm{H}$. pylori presence. Risks associated with taking a biopsy of the wall of the ulcer can thus be avoided.

\section{O169}

\section{NEOADJUVANT RADIOTHERAPY SUPPRESSES LEUCOCYTE COUNTS IN RECTAL CANCER TREATMENT: A TWO CENTRE STUDY}

L.B. Johnson ${ }^{1}$, B. Ottochian ${ }^{1}$, C. Albertsson ${ }^{1}$, D. Adawi', J. Manjer ${ }^{1}$, E. Zoucas ${ }^{2}$, M. Bohe' ${ }^{1}$, B. Jeppsson ${ }^{1}$

Lund University Hospital, Lund, Sweden

Introduction: Impaired postoperative leucocytosis has been suggested as being the result of neoadjuvant radiotherapy in the treatment of operable rectal cancer disease. An earlier retrospective non-randomised study from Malmö ' Sweden has shown a reduction in peripheral leucocyte counts in the first postoperative week, after both short and long term radiotherapy. A correlation between the level of post / preoperative leucocyte count and improvement of survival was observed in the non-radiated group. This study is an extension of the Malmö study and includes patients from both Lund and Malmö University Hospitals in an effort to see if the same is true for the extended study group.

Methods: 906 files from rectal cancer patients treated in the above study groups between 1993 ' 2005 were examined. Pre-postoperative leucocyte counts, complications from files and causes of deaths from the national cancer register of Sweden were noted down. The initial statistical analysis of variations in leucocyte counts before and after radiotherapy as well as 1 week after surgery was done.

Results: A significant reduction $(p<0,05)$ of preoperative leucocyte counts after both short and long term radiotherapy was observed compared to the surgery only group. Both radiotherapy groups showed a significant reduction in the $24 \mathrm{hr}$ postoperative leucocytosis. Only the short-term radiotherapy group had a significantly reduced leucocyte count in the first postoperative week compared to surgery alone. Conclusion: Both long and short term neoadjuvant radiotherapy reduces significantly preoperative leucocyte levels. Whilst short term radiotherapy alone results in the maintenance of a significantly reduced leucocyte count in the first postoperative week.

O170

\section{CLINICAL OUTCOME IN INCARCERATED HERNIAS}

J. Nieuwenhuizen, D.D. Krijg, G.H. van Ramshorst, W.C.J. Hop, J. Jeekel, J.F. Lange

Erasmus Medical Center Rotterdam, Rotterdam, The Netherlands

Background: Incarceration and/or strangulation of inguinal, umbilical en cicatricial hernias is a frequent and sometimes lethal problem. However, little is known about the relation between clinical presentation and findings during surgery. Goal of this study was to describe the relation between clinical presentation of patients with an incarcerated hernia and the per- and post-operative findings.

Methods: Correspondence, operation reports and patient files between January 1995 and December 2005 of patients presented at the Surgery Department in the Erasmus Medical Centre in Rotterdam were searched for the words: incarceration, strangulation and hernia. Patient characteristics, clinical presentation, per-operative findings and clinical course were scored and analyzed.

Results: One hundred and forty eight patients could be identified with a suspected incarcerated hernia. Ten patients appeared not to have an incarcerated hernia per-operatively, 2 patients were transferred to an other clinic and 10 patients received an elective repair after reduction of the hernia leaving 126 patients for further analysis. Eventually 40 inguinal, 42 umbilical, 7 epigastric, 31 cicatricial, 4 femoral and 2 trocar hernias were analyzed. In 26 patients $(20.6 \%)$ it was a recurrent hernia. Of these patients $61.9 \%$ was male $(n=78)$ and $38.1 \%$ was female $(\mathrm{n}=48)$. Age varied between 24 and 89 years (mean 59.9 years). At presentation $86.6 \%$ had an irreducible swelling, $48.7 \%$ nausea, 39.3 $\%$ vomited and $29.9 \%$ had sings of bowel obstruction. In $48.7 \%$ the hernia sac contained colon or small intestine. Bowel resection was performed in 19 patients $(16.0 \%)$. Complications were seen in $35.3 \%$ of all patients. Mortality was $6.3 \%(n=8)$. Patients presenting with nausea $(p=0.03$, OR 3.3 ) or have signs of bowel obstruction ( $p=0.03$, OR 4.0) or have an ASA class $>1(p=0.01$, OR 11.4) are more likely to have a hernia sac containing small or large bowel. Signs of bowel obstruction was the only predictor for bowel resection $(p=0.01$, OR 6,2$)$. ASA class $>1(p=0.03$, OR 4.8), female gender $(p=0.01$, OR 3.9$)$, and signs of an ileus ( $p=<0.01$, OR 5.7) were risk factors for developing complications. Hernia type was not a predictor of outcome.

Conclusion: Incarcerated hernias are associated with high morbidity $(35 \%)$ and high mortality $(6 \%)$. Type of hernia can not predict the outcome. Main predictors of clinical outcome are signs of bowel obstruction and ASA-class.

O171

THE EFFECT OF ANTI-OXIDANT ENRICHED ENTERAL NUTRITION ON THE IMMUNO-INFLAMMATORY RESPONSE AFTER MAJOR UPPER GASTRO-INTESTINAL TRACT SURGERY. A PROSPECTIVE RANDOMIZED CONTROLLED TRIAL

M.F.M. van Stijn', P.G. Boelens ${ }^{2}$, M.C. Richir², G.C. Melis-Lighthart², J. Diks ${ }^{2}$, M.A. Cuesta'2, B.M.E. Von Blomberg-van der Flier'², W. Buurman $^{3}$, P.A.M. van Leeuwen ${ }^{2}$

1 VU Medical Center, Amsterdam, The Netherlands

2 VU University Medical Center, Amsterdam, The Netherlands

3 University Medical Center Maastricht, Maastricht, The Netherlands 
Background: Major surgery and (subsequent) critical illness induce an immuno-inflammatory response, which is accompanied by production of reactive oxygen species (ROS) at the site of the injury. Endogenous anti-oxidants are needed to prevent damage of overproduction of ROS. Previous studies show that plasma concentrations of specific amino acids and anti-oxidant micronutrients (zinc, vitamin E, vitamin C, betacarotene, selenium) markedly decrease after major surgery. These deficiencies may not be sufficiently covered by the composition of standard enteral nutrition. Therefore, the aim of this study was to evaluate the effect of an anti-oxidant enriched nutrition on the immuno-inflammatory response after major upper gastro-intestinal tract surgery.

Methods: A prospective RCT at the surgical intensive care and surgical ward of the VU University Medical Centre. Twenty-one patients, with tumours of the upper gastro-intestinal tract, were included and studied during the week after undergoing major upper gastro-intestinal tract surgery, with operative procedures of at least 3 hours. After surgery patients received a standard enteral nutrition (Sondalis ISO, Nestlé, Swiss) by jejunostomy with (treatment group, $\mathrm{n}=11$ ) or without (control group, $\mathrm{n}=10$ ) a supplement containing anti-oxidants or their precursors (glutamine, cysteine, zinc, vitamin E, vitamin C, beta-carotene, selenium). Immunological and inflammatory markers were determined before surgery and on day 1, 3, 5 and 7 after surgery.

Results: Both patient groups were comparable with respect to anthropometrics, surgery and tumour characteristics. The inflammatory markers IL-6, soluble p55/60 TNF receptor, LBP and CRP increased due to surgery, in both the control and treatment group. Surgery reduced HLA-DR expression on CD14+ monocytes in both groups $(p<0.05)$. The concentration of leucocytes, BPI and elastase increased significantly in the treatment group, while IL-8, soluble p75/80 TNF receptor and MBL increased significantly in the control group. The anti-oxidant enriched nutrition reduced CRP in the first 7 days $(p<0.05)$ after supplementation. Also soluble $p 75 / 80$ TNF receptor significantly decreased in the first three days $(p<0.05)$ in the treatment group as compared to the control group. LBP decreased significantly in the treatment group as compared to the control group $(p<0.05)$.

Conclusion: Major upper gastro-intestinal tract surgery induces a systemic immuno-inflammatory response with reduced HLA-DR expression, increased concentrations of IL-6, IL-8, soluble TNF receptors, elastase, $\mathrm{BPI}, \mathrm{MBL}, \mathrm{LBP}$ and CRP. Enteral nutrition enriched with glutamine and anti-oxidants is a safe and well-supplement to standard enteral nutrition that blunted significantly the inflammatory response (CRP, soluble p75/80 TNF receptor and LBP) induced by surgery.

0173

ONE ANASTOMOSIS GASTRIC BYPASS BY LAPAROSCOPY: RESULTS AFTER THE LERANING CURVE

\author{
G.C.M. Garcia-Caballero'1, M. Carbajo², D. Osorio ${ }^{1}$, A. Minguez', \\ C. Garcia-lanza ${ }^{2}$ \\ 1 University Malaga, Malaga, Spain \\ 2 Hospital Ampo Grande, Valadolid, Spain
}

Background: The One Anastomosis Gastric Bypass by laparoscopy (OAGB) is an european procedure for treating morbid obesity. It consists of making a $25 \mathrm{ml}$ gastric pouch between the esophago-gastric junction and the crow's foot level, parallel to the short curvature which is anastomosed latero-lateraly to a jejunal loop $2 \mathrm{~m}$ distal from the ligament of Treitz. We compared the results of the 209 first patients with those of the next 209 (210-418).

Methods: Both groups are comparable in age, BMI and pre-operative excess body weight as well as associated surgical procedures and redo of previous restrictive bariatric procedure.

Results: In the first 209 patients, we converted two patients $(0.9 \%)$ to open surgery due to uncontrollable bleeding. In three cases $(1.4 \%)$ the patients needed re-operation in the immediate postoperative period. Five patients $(2.3 \%)$ needed a prolonged hospital stay due to acute pancreatitis in one and four others had an anastomotic leakage, all resolving with conservative treatment. Two patients died $(0.9 \%)$, one due to fulminant pulmonary thromboembolism and one had a nosocomial pneumonia. In next 209 (210-418) there were only one case of perforation in the esophago-gastric junction $(0.4 \%)$ and one case of intestinal obstruction distal to gastro-jejunal anastomosis $24 \mathrm{~h}$ after operation $(0.4 \%)$.

Conclusion: These results demonstrated that OAGB is a procedure that induced a important weight loss with minimal complications.

\section{SIRNA TREATMENT FOR ESOPHAGEAL CANCER CELLS}

Y. Shimada ${ }^{1}$, Y. Hashimoto 2 , T. Ito ${ }^{3}$, T. Okumura ${ }^{4}$, E. Tanaka ${ }^{5}$, Y. Mori ${ }^{5}$, S. Tsunoda ${ }^{6}$, C. Ortiz ${ }^{5}$, J. Fujimoto ${ }^{1}$

1 Hyogo College of Medicine, Nishinomiya, Japan

2 Clevelamd Clinic, Cleveland, USA

3 Johns Hopkins University, Baltimore, USA

4 Columbia University, New York, USA

5 Kyoto University, Kyoto, Japan

6 University of Adelaide, Adelaide, Australia

Introduction: Previous results suggested that expression of Fascin, Osteopontin (OPN), Aurora A/STK15/BTAK, p75/NTR, NGF, Gli-1 and Gankyrin associated with lymph node metastasis and the prognosis of patients with esophageal cancer. To evaluate whether targeting of these molecules could suppress the aggressive behavior of the esophageal cancer, we tried to knock these molecules down using SiRNA

Methods: We made specific SiRNA for Fascin, OPN, Aurora A/STK15/ BTAK, p75/NTR, NGF, Gli-1 and Gankyrin, Then, we examined the inhibitory effect of SiRNA in the esophageal cancer cell lines (KYSE-series). Results: Molecular specific SiRNA markedly inhibited the motility, invasiveness and survival of esophageal cancer cell lines ranging from $40 \%$ to $97 \%$ compared to non treated cells and nonspecific control SiRNAtreated cells. Furthermore, SiRNA for Aurora-A/STK15/BTAK expression enhances chemo-sensitivity to Docetaxel (44\% tumor growth suppression) in esophageal squamous cell carcinoma cells.

Conclusion: Our results suggested that Fascin, OPN, Aurora A/STK15/ BTAK, p75/NTR, NGF, Gli-1 and Gankyrin may be a good target for treatment of esophageal cancer patients. For clinical application of SiRNA, drug delivery, cost and off target effects are the problems we are now facing.

\section{O175}

POLYMORPHONUCLEAR NEUTROPHIL DERIVED MMP-9 AND TUMOR CELL DERIVED VEGF ARE INDEPENDENT PRO-ANGIONENIC FACTORS IN AN IN-VITRO ANGIOGENESIS MODEL OF PANCREATIC CARCINOMA

D. Bausch, T. Keck, U.T. Hopt

University Clinic Freiburg, Freiburg, Germany

Introduction: Pancreatic carcinoma shows strong tumor neoangiogenesis. One key mediator of angiogenesis is vascular endothelial growth factor (VEGF), which is overexpressed in pancreatic carcinoma. Another important angiogenic co-factor is matrix metalloprotease 9 (MMP-9), derived from peritumoral stroma and peritumoral inflammation mediated by polymorphonuclear neutrophils (PMN). PMN act early in the angiogenic cascade and their action seems to be VEGF dependant. We studied the angiogenic role of MMP-9 derived from PMN and the ability to block its action using tetracyclines as MMP inhibitors during tumor angiogenesis in an in vitro model of pancreatic cancer using a human vascular endothelial cell (HUVEC) spheroid based sprouting assay and CAPAN-1 human pancreas cancer cell line.

Methods and materials: HUCVEC were freshly isolated from human umbilical veins by collagenase digestion. To generate endothelial cell spheroids, HUVEC were suspended in culture medium containing carboxymethylcellulose and seeded in hanging drops. The spheroids were harvested within $24 \mathrm{~h}$ and embedded into collagen gels. The test substances and/or cells were then added on top of the gels. To quantify in-gel angiogenesis, the cumulative length of all capillary like sprouts originating from the central plain of an individual spheroid was measured after $24 \mathrm{~h}$. At least 10 spheroids per experimental group and experiment were analyzed. To analyze the expression of VEGF and MMP-9 in HUVEC, PMN and CAPAN-1 cells quantitative Western blot analysis was performed.

Results: Quantitative Western blot analysis of CAPAN-1, PMN and HUVEC for MMP-9 and VEGF demonstrated no significant MMP-9 production in HUVEC and CAPAN-1, whereas the pro-form of the protein was found in abundance in PMN. VEGF could only be found in CAPAN-1. VEGF and MMP-9 alone induced significant angiogensis and the combination of both proteins resulted in significantly increased angiogenesis. CAPAN-1 and PMN alone also stimulated significant angiogensis and their combination resulted in more angiogenesis than each single cell type alone could induce. The combination of CAPAN-1 and VEGF or PMN and MMP-9 respectively lead to no further angiogenesis. Antibodies to VEGF were able to abolish angiogenesis in VEGF and CAPAN stimulated spheroids, but not in MMP-9 stimulated HUVECS whereas 
antibodies to MMP-9 abolished angiogenesis in MMP-9 and PMN stimulated spheroids. Tetracycline action was similar to antibodies to MMP-9. Combination of VEGF with MMP-9 antibodies reduced angiogenesis to about the level of the other stimulant. The same effect was observed for the combination of CAPAN-1 and PMN whereas tetracyclines acted as antibodies to MMP-9. The combination of both antibodies or the combination of tetracyclines and antibodies to VEGF completely abolished angiogenesis in the protein and cell experiments.

Discussion: This study demonstrates that PMN derived MMP play a role in tumor angiogenesis in an in vitro model of pancreatic cancer and that the combination of different anti-angiogenic agents is feasible. Additionally, the MMP-9 dependent anti-angiogenic properties of tetracyclines were demonstrated. Surprisingly, a VEGF independent pro-angiogenic effect of MMP-9 could be found. Inhibition of peritumoral inflammation around pancreatic cancer cells might prove to be a efficient strategy to reduce angiogenesis driven by peritumoral stomal inflammation.

\section{O176}

\section{LYMPHANGIOGENESIS OR ONLY DILATATION OF LYMPHATICS IN} COLON CANCER?

M. Stanczyk ${ }^{1}$, W.L. Olszewski ${ }^{1}$, M. Gewartowska ${ }^{1}, M^{2}$. Cakala¹,

M. Maruszynski ${ }^{2}$

1 Medical Research Center, Warsaw, Poland

2 Military Health Service Institute, Warsaw, Poland

Introduction: The extent of lymph node metastasis is a major determinant for the staging and the prognosis of most human malignancies. Although the clinical significance of lymph node involvement is well documented, molecular mechanisms that promote tumor spread into the lymphatic or blood vascular systems and widespread dissemination are not well understood. Although there is a large body of evidence that newly visualized lymphatics facilitate formation of metastases, it remains unclear whether these are 'new' or simply pre-existing dilated vessels. High level of permeability of tumor blood capillaries brings about high tissue fluid and lymph formation. The physical forces but not the putative cancer-produced VEGF C may be responsible for more lymphatics seen around cancer than in normal tissue. The main question to be answered is: are there morphologic and functional differences between newly formed and pre-existing intra- or peritumoral lymphatics?

Aim: The aim of our study was to visualize tissue space and initial and collecting lymphatics in and around gastric and colon cancer foci.

Methods: Samples of human gastric and colon cancer G2 and normal gastric and colon tissue, were obtained from 12 patients. Tissue samples were divided into two fragments. One was injected with Patent Blue in chloroform suspension to visualize interstitial space and lymphatic capillaries. Large particles of this dye enter open lymphatics but nor tightwall blood capillaries. Another fragment was sectioned and stained with monoclonal antibodies for CD68, CD3, elastase, LYVE1, Prox1, podolanin, CD31, ICAM1, VCAM1. The number, topography and morphology of lymphatic vessels were evaluated in the peri- and intratumoral areas. Results: The peritumoral area contained multiple wide-lumen lymphatic collecting vessels. A dense network of these vessels was seen in some parts of the specimen. These vessels stained positively with mAbs againstLYVE1 and CD31 but not Prox1, podoplanin, ICAM1 and VCAM1. In some vessels single large cells resembling tumor cells could be noticed. In the intratumoral areas no lymphatics were seen. However, thousands of minute 'lakes' could be visualized what was not observed in a normal wall. This pattern is usually seen in tissues with lymphedema. These irregular tissue fluid spaces could arise due to high capillary permeability

Conclusion: Specimens of gastric and colon cancer revealed presence of peri-tumoral but not intra-tumoral lymphatics. Tumor tissue contained numerous tissue fluid 'lakes' communicating with lymphatics. We speculate that increased production of lymph in the tumor tissue caused by high blood capillary permeability brings about dilatation of the interstitial space and lymphatics. Excessive lymph flow may drag tumor cells.

\section{O177}

CHRONIC SOFT TISSUE INFECTIONS STIMULATE FORMATION OF NEW LYMPH NODES

W.L. Olszewski ${ }^{1}$, J. Cwikla ${ }^{2}$

1 Medical Research Center, Warsaw, Poland

2 Nuclear Medicine Laboratory, Warsaw, Poland
Organized lymphoid tissue is the first line of defense against foreign and self-antigens. Recruited by antigen located in the non-lymphoid tissues, the infiltrating lymphocytes often organize themselves as follicle-like structures containing germinal centers similar to those in secondary lymphoid follicles of lymph nodes. These ectopic or extranodal tertiary lymphoid follicles are found in various autoimmune diseases.

Aim: To detect by means of isotopic lymphography formation of new lymphatic tissue.

Materials \& methods: We investigated a group of 153 patients with long-lasting lymph stasis with edema in lower limbs, caused by damage to lymphatics either by soft tissue bacterial inflammation or mechanical trauma of soft tissues and bones. The $99 \mathrm{~m}$ Tc-labelled Nanocoll (aggregated albumin) was injected intradermally into foot toe-web and spread of radioactivity followed using a gamma-camera. The time of absorption of the tracer, its flow to inguinal lymph nodes, accumulation in inguinal nodes and lymph nodes along the lymphatic collaterals and deep vessels was monitored.

Results: In $12.5 \%$ of patients with postinflammatory and $18 \%$ with posttraumatic lymph stasis 'newly-formed' lymph nodes were detected by means of lymphoscintigraphy. They were located along the large calf and thigh veins. Although some few nodes are normally detected in these areas (less than $5 \%$ ), the number and total mass of visualized nodes largely exceeded those seen in healthy subjects. The calculated surface area of 'newly-formed' nodes reached $50-70 \%$ of the area of ipsilateral inguinal nodes. Histological evaluation of specimens of biopsied nodes of 3 patients revealed in one a lymph node structure without differentiation into cortical and medullary area, in another a follicle-like structure in a dilated lymph vessel. Lymph clot removed from another dilated vessel contained a lymphocyte/dendritic cell aggregate.

Conclusion: The 'newly-formed' nodes most likely originate from primordial lymphoid follicles and/or lymphoid cell aggregates formed in response to chronic stimulation by microbial products and self-antigens from the damaged tissues. Detection of 'newly-formed' lymph nodes in the limb is a sign of a continuing inflammatory process and requires appropriate therapy. Our studies are the first ever reported on lymphoid tissue neo-formation in human limbs.

\section{8}

RETINOIC ACID TO CONTROL RECURRENCE FOLLOWING EXPERIMENTAL LIVER METASTASES RESECTION

\author{
I. Garcia-Alonso, T. Palomares, J. San Cristobal, I. Cearra, R. San \\ Isidro, T. Martinez-Astorquiza \\ Faculty of Medicine (UPV/EHU), Lejona, Spain
}

In some experimental models, we showed that partial hepatectomy for tumour resection increased the number of metastasis appearing in the remaining liver in the following weeks. It seems very likely that something similar may occur in clinical situations. Finding a treatment that reverse this 'tumour promoting' effect without affecting recovery from surgery is our aim.

Methods: Wag male rats were used. The liver was seeded with syngenic tumour cells (rhabdomyosarcoma, $\mathrm{S} 4 \mathrm{MH}$ cell line) by inoculating 250,000 cells into the spleen. To avoid a 'primary' tumour, the spleen is removed 5 minutes later. On day 10th a $40 \%$ hepatectomy is performed. All-trans-retinoic acid (ATRA) is administered i.p. ( $5 \mathrm{mg} / \mathrm{kg}$ ) starting on day 7 th.

Results: Daily doses of ATRA during 2 weeks halved the mean number of liver metastases ( $3.1 \mathrm{vs} 6.8, p<0.01$ ) at the end of the treatment and significantly prolonged the survival period, though all of the animals died before day 80th. Partial hepatectomy in rats induces a certain degree of anaemia: haemoglobin 14.9 vs $13.0(p=0.001)$, erythrocytes 7.8 vs 6.7 $(p=0.0006)$, haematocrit 43.6 vs $39.5(p<0.05)$, but it is not affected by ATRA treatment (nor it is increased, neither the recovery is hindered). When partial hepatectomy is performed in ATRA treated animals, a sharp drop in leucocytes numbers is constantly induced $(8,000$ vs 2,800 ; $p<0.001$ ), recovering normal figures on day $22 n d$. Partial hepatectomy increases serum levels of alkaline phosphatase (AP), aspartate transaminase (AST) and lactate dehydrogenase (LDH). ATRA treatment further increases AP on day $22 n d(244$ vs $136, p<0.05)$, and AST shows a mild elevation ( 126 vs $103, p=0.05$ ). If ATRA treatment is shortened to 7 days, the effect on the number of liver metastases is not affected but the increase in AP and AST is avoided.

Conclusion: A short treatment with ATRA, starting a few days before liver resection could be useful to block the stimulating effect of surgery on tumour cell growth, but it does not prevent the late progression of the 
metastases and only accounts for a delay in the death of the animals. The effect of repeated short courses of ATRA treatment should be explored in order to achieve a grater expanse of the survival period.

\section{O179}

THE EFFECTS OF PRE-OPERATIVE ADMINISTRATION OF CPG 7909 ON DENDRITIC AND T CELL SUBSETS IN THE SENTINEL LYMPH NODE OF STAGE I/II MELANOMA PATIENTS

B.J.R. Sluijter, B.G. Molenkamp, T.D. de Gruijl, P.G.J.T.B Wijnands, A.J.M van den Eertwegh, R.J. Scheper, S. Meijer, P.A.M. van Leeuwen VUMC Amsterdam, Amsterdam, The Netherlands

Background: A decrease in the frequency and activation state of Dendritic Cells (DC) in the Sentinel Lymph Node (SLN) has been observed in early stages of melanoma development, which may impair the generation of effective anti-tumor $T$ cell responses and increase the likelihood of metastatic spread. Bacterially derived unmethylated CpG oligodeoxynucleotides (ODN) bind the Toll-like receptor-9 and activate human plasmacytoid DC (PDC), which in turn release IFNa and may thus activate myeloid $\mathrm{DC}$ (MDC) and boost $\mathrm{T}$ cell responses. We studied the effects of the CpG-B ODN PF-3512676 (formerly CpG 7909) on the activation state of PDC and MDC as well as on the frequency of regulatory T cells (Treg) and tumor-specific CD8+ T cells in the SLN.

Methods: Patients with clinically Stage-I/II melanoma, who were scheduled to undergo a SLN procedure, were assigned randomly to preoperative local administration of either PF-3512676 $(n=11)$ or saline $(n=12)$. Both patient groups received an i.d. injection adjacent to the scar of the primary melanoma excision a week before operation. SLN were bisected and the cutting surface scraped to obtain viable immune effector cells for functional analyses, prior to pathological examination. Results: PF-3512676 administration resulted in significantly bulkier SLN and flowcytometric analysis clearly demonstrated maturation induction of BDCA-2+CD123+ PDC by de novo expression of CD83 and CD86 and increased activation of CD1a+ MDC by increased expression levels of the same markers. In addition, PF-3512676 administration was associated with the presence of a novel CD11chiCD123+CD83+ mature SLN-MDC subset, further characterized by the absence of CD1a or CD14 and the expression of TRAIL, CCR7, and high levels of costimulatory molecules. PF-3512676 administration was also associated with significantly lower CD4+CD25hi Treg frequencies $(P=0.004)$ and increased CD8+ $T$ cell reactivity rate against a range of melanoma-derived epitopes in an IFNa ELISPOT read-out (5/10 responders versus $0 / 11$ in the saline control group, $\mathrm{P}=0.02$ )

Conclusion: PF-3512676 is tolerated well and exerts immunostimulatory effects on DC and T cell subsets in the SLN with possible therapeutic benefit in early stages of melanoma development.

\section{O180}

\section{TUMOUR METABOLISM IN COLORECTAL CANCER AND LIVER} METASTASES: A REVISITATION OF THE WARBURG EFFECT

\author{
I.A. Ansari', R. Rajaganeshan¹, N. Scott ${ }^{2}$, I. Ansari ${ }^{1}$, P.J. Guillou², \\ D.G. Jayne ${ }^{2}$ \\ 1 University of Leeds, School of medicine, Leeds, United Kingdom \\ 2 St James's University Hospital, Leeds, United Kingdom
}

Background: A link between mitochondrial dysfunction and tumour glycolysis was proposed by Warburg in the 1930's. The current study aimed to investigate this association with specific reference to mitochondrial cytochrome c oxidase (COX).

Methods: Tissue microarray immunohistochemistry was used to investigate the expression of mitochondrial DNA-encoded subunit II and nuclear DNA-encoded subunit IV of COX in parallel with markers of glucose uptake and glycolysis (GLUT-1, hexokinase II and LDH 5) in 79 colorectal cancers and 53 liver metastases (local ethical committee approved).

Results: A large variation in COX subunit expression was observed. In $44 / 79(56 \%)$ primary cancers, COX II and IV expression was moderatestrong, with selective suppression of COX II in $22 / 79(28 \%)$ cancers $(p<0.0001)$. Intense glycolysis was evident in $83 \%$ of tumours with moderate-strong expression of both COX subunits, compared to only $40 \%$ in COX II-suppressed tumours $(p=0.001)$. At the invasive margin, COX I and IV were found to positively correlate with hexokinase II $(p=0.02$ and $p=0.016)$ and LDH $5(p<0.0001$ and $p=0.001)$.

Kaplan Meier analysis showed that increased COX II expression was significantly associated with liver metastases [mean $(95 \% \mathrm{Cl})$, high COX II: 43.9(32.9-54.9) vs. Iow COX II: 67.4(54.21-80.51); Log rank $p=0.026]$. Conclusion: Selective suppression of COX II is associated with reduced glycolytic activity and reduced metastatic potential. The observed relationship between mitochondrial and glycolytic activity is in direct contrast to that proposed by Warburg and raises important questions concerning tumour metabolism.

Abbreviations: COX, cytochrome c oxidase; GLUT-1, glucose transporter-1; LDH 5, lactate dehydrogenase 5.

\section{O181 \\ DETERMINATION OF DIFFERENTIALLY EXPRESSED ANGIOGENIC FACTORS BY MALIGNANT MELANOMA}

T.L.M. ten Hagen, L. Kwee, C.E. Vermeulen, J. Rens, A.M.M. Eggermont Erasmus Medical Center Rotterdam, Rotterdam, The Netherlands

The population of patients with malignant melanoma is one of the most rapidly increasing cancer groups worldwide. Because current therapeutic methods for metastatic melanoma are poorly efficacious, enhanced understanding of melanoma development is required. Previous research has shown a possible correlation between the degree of vascularization of melanoma and their metastasizing capacity. Inhibition of angiogenesis, the development of new blood vessels out of pre-existing ones, could therefore be a potential therapy. Tumor cells initiate the onset of new blood vessel formation by releasing VEGF and numerous other angiogenic factors. In this study we focused on melanoma-mediated endothelial cell survival. Six human melanoma cell lines, which differ in degree of aggressiveness, were cultured under normoxic (20\%O2) and hypoxic $(1 \% \mathrm{O} 2)$ conditions, since hypoxia strongly induces angiogenic factor production. Melanoma-conditioned medium of all cell lines was capable of augmenting endothelial cell survival after hypoxia. The activity of VEGF and the determination of differentially expressed pro- and anti-angiogenic factors by the selected melanoma cells were determined by means of RT-PCR, ELISA and Affymetrix-based microarray analyses. Clearly, the melanoma-induced endothelial cell survival was not solely dependent on VEGF. Our observations suggest that, instead of focusing on a single factor, efficient anti-angiogenic treatment of melanoma may require identification and blocking of several factors.

\section{4}

\section{ANTI-INFLAMMATORY EFFECTS OF ORAL PHOSPHATIDYLETHA-} NOLAMINE IN CARRAGEENAN INDUCED PLEURISY

\section{G. Eros ${ }^{1}$, G. Varga ${ }^{2}$, R. Vradi ${ }^{1}$, M. Czbel ${ }^{1}$, J. Kaszaki $^{1}$, M. Boros ${ }^{1}$ 1 University Of Szeged, Szeged, Hungary, Hungary}

Introduction: Phosphatidylethanolamines (PEs) are endogenous molecules linked to the phosphatidylcholine (PC) metabolism. In this pathway, a fatty acyl chain of membrane glycerophospholipids is transferred to phosphatidylethanolamine, resulting in the generation of endocannabinoid precursors. Our previous studies have shown that modulation of PC input may inhibit the inflammatory consequences of several pathologies: PC treatment alleviated the inflammatory complications of bile-induced esophagitis, tissue ischemia-reperfusion and endotoxemia. The objective of our current studies was to examine the consequences of exogenous PE administration in a rat model of subacute inflammation, and to characterize the efficacy of oral PE administration as compared with a generally-used non-steroid anti-inflammatory drug. Methods: The experiments were performed on male CFLP mice (average weight $25 \pm 5 \mathrm{~g}$ ) under sodium pentobarbital anaesthesia. In group 1 $(n=8) 0.1 \mathrm{ml}$ of saline, in groups $2-4(n=8$, each) $0.1 \mathrm{ml}$ saline containing $2 \% \lambda$-carrageenan was injected into the pleural cavity. In group 3 , the animals were fed ad libitum with PE-enriched diet (Ssniff Spezialdiäten, Ssniff $\mathrm{GmbH}$, Cologne, Germany) for 7 days before- and for 3 days after carrageenan administration. Group 4 received acetyl-salicylic-acid (ASA) in drinking water $(1.5 \mathrm{mg} / \mathrm{ml})$ for 3 days after pleuritis induction. The animals were sacrificed 3 days after the operation, and leukocytes in the pleural cavity were counted by means of toluidine blue staining, while the activities of xanthine oxidoreductase (XOR) and myeloperoxidase (MPO) were measured on lung tissue biopsies.

Results: Carrageenan increased the number of intrapleural leukocytes by $\sim 160 \%$, while the activities of MPO and XOR were elevated by $82 \%$ and $60 \%$, respectively. Oral PE pre-treatment prevented these changes significantly, the reduction in leukocyte counts and inflammatory enzyme activities was comparable to that observed after ASA treatment. 
Conclusion: Oral PE pre-treatment effectively inhibits leukocyte activation and accumulation in the rat pleuritis model. The PE pathway could be a pharmaco-therapeutic target in inflammatory pathologies.

\section{5}

\section{TC-GSA SCINTIGRAPHY WITH SPECT IN THE ASSESSMENT OF HEPATIC FUNCTION AND FUNCTIONAL VOLUME DURING LIVER REGENERATION IN A RAT MODEL}

W. de Graaf, R.L. Vetelainen, A.K. Van Vliet, R.J. Bennink, T. M. Van Gulik Academic Medical Center Amsterdam, Amsterdam, The Netherlands
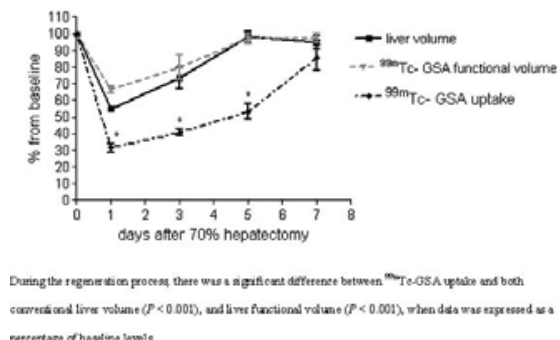

Small animal models are crucial in order to gain insights in the complex recovery mechanisms of liver function during liver regeneration. Many quantitative liver function tests require repetitive blood samples, and are therefore difficult to use in small animals. 99m Tc-mebrofenin hepatobiliairy scintigraphy (HBS) has been introduced for non-invasive assessment of liver function in the clinical setting as well as in experimental research. However, HBS is restricted to planar modalities in small animals since hepatic kinetics are generally too fast for SPECT acquisition. 99m Tc-GSA scintigraphy is an alternative, receptor mediated, non-invasive liver function test. After hepatic uptake, 99m Tc-GSA remains trapped in the liver enabling additional SPECT for the assessment of both liver function and liver functional volume within one test. In this study, the use of 99m Tc-GSA scintigraphy combined with SPECT for the assessment of liver function and liver functional volume in normal and regenerating rat liver was evaluated. Reproducibility of $99 \mathrm{~m}$ Tc-GSA scintigraphy and SPECT was investigated by repeated measurements within the same rat. For the assement in a regenerating liver, liver function (<99m Tc-GSA uptake), liver functional volume by $99 \mathrm{mTc}-\mathrm{GSA}$ SPECT and conventional liver volume was assessed on 1, 3, 5 and 7 days ( $n=6$ per time point) after $70 \%$ partial hepatectomy $(\mathrm{PH})$. The correlation between repeated (99m Tc-GSA measurements was significant and strong $(r=0.754, p=0.019)$. In normal rat livers, there was a strong and significant correlation between functional volume assessed by $(99 \mathrm{~m}$ TC-GSA SPECT and conventional liver volume $(r=0.93, p<0.0001)$. Correlation between $(99 \mathrm{~m}$ Tc-GSA uptake and conventional liver volume was moderate $(r=0.62, p=0.043)$. The mean liver functional volume and conventional liver volume was significantly $(p<0.05)$ decreased 1 day after $70 \% \mathrm{PH}(8.07 \pm 0.27 \mathrm{ml}$ and $6.77 \pm 0.12 \mathrm{ml}$, respectively) compared to baseline $(12.14 \pm 0.20 \mathrm{ml}$ and $12.38 \pm 0.32$, respectively), after which it regenerated to normal liver volume at day $5(11.82 \pm 0.39 \mathrm{ml}$ and $12.15 \pm 0.45 \mathrm{ml})$ and day $7(11.80 \pm 0.37 \mathrm{ml}$ and $11.76 \pm 0.50 \mathrm{ml})$. There was a strong correlation between the functional volume and conventional volume in the regenerating liver $(r=0.86, p<0.0001)$. One day after $70 \% \mathrm{PH}$, the $(99 \mathrm{~m}$ Tc-GSA uptake $(8.01 \pm 0.62 \% / \mathrm{min})$ significantly decreased compared to baseline $(27.08 \pm 1.82 \% / \mathrm{min})$, after which it slowly increased to baseline level at day $7(21.71 \pm 1.94 \% / \mathrm{min})$. During the regeneration process $(99 \mathrm{~m}$ Tc-GSA uptake was significantly lower compared to both conventional volume $(p<0.001)$, and functional volume ( $p$ $<0.001$ ), when expressed as a percentage of baseline levels

(99m Tc-GSA scintigraphy combined with SPECT is a feasible, noninvasive method for the assessment of hepatic functional volume in normal, as well as in regenerating rat livers. However, the hepatic $(99 \mathrm{~m}$ Tc-GSA uptake as a liver function test seems to underestimate the hepatic regeneration.

\section{O186}

INFLAMMATORY CHANGES AFTER SHORT-TERM ISCHEMIC PRECONDITIONING OF THE RAT HINDLIMB

R. Varga ${ }^{1}$, R. Varga ${ }^{2}$, A. Szab², M. Keresztes ${ }^{3}$, J. Kaszaki², I. Németh ${ }^{4}$, Z. Razga', M. Boros ${ }^{2}$

1 University Of Szeged, Szeged, Hungary
2 Institute Of Surgical Research, Szeged, Hungary

3 Institute Of Biochemistry, Szeged, Hungary

4 Institute Of Pathology, Szeged, Hungary

Introduction: Ischemic preconditioning (IPC) may ameliorate local and remote consequences of subsequent ischemic insults, and leukocytemediated processes are implicated in this protective response. The effects of IPC on periosteal microcirculation, however, are not yet clarified. In this study, we examined the inflammatory consequences of an IPC protocol in a rat model of limb ischemia by characterizing local neutrophil-endothelial interactions in the periosteum and the expression of different adhesion molecules which are thought to play a role in leukocyte-mediated inflammatory processes.

Methods: In group 1, IPC (2 cycles of 10 min complete limb ischemia and $10 \mathrm{~min}$ reperfusion) was induced, while group 2 was subjected to sham operation. In groups 3 and 4 , ischemia-reperfusion alone (I-R, $60 \mathrm{~min} / 180 \mathrm{~min}$ ) or with IPC (IPC+I-R) was performed. Postischemic neutrophil-endothelial interactions in the tibial periosteum were quantified by means of fluorescence intravital videomicroscopy. Intravascular neutrophil activation was assessed by the increase in the expression of adhesion molecule CD11b (measured with FACS analysis using B\&D Pharmingen FITC-labeled mouse anti-rat antibody) and expressed as changes in mean fluorescence intensity over the baseline. Soluble ICAM-1 expression (ELISA, R\&D Systems) and tissue ICAM-1 expression (detected by immunohistochemistry, B\&D Pharmingen mouse antirat antibody, SABC technique) were also assessed. Tissue deposition of neutrophils was quantified by myeloperoxidase (MPO) assay.

Results: IPC alone did not significantly influence the examined parameters. I-R alone induced an enhanced leukocyte adherence to the periosteal venules after the 120th and 180th min of reperfusion (the number of sticking cells increased from approx. 210 to 440 and $460 \mathrm{~mm}-2$, respectively) which was associated with an increased CD11b expression (by approx. $80 \%$ and $65 \%$, respectively) and elevated sICAM- 1 levels (by approx. $30 \%$ and $45 \%$, respectively). IPC prevented the I-R-induced increases in leukocyte adherence and CD11b expression without influencing sICAM-1 levels. MPO increased after I-R by approx. $30 \%$ in the lungs and also in the affected muscle (by approx. 30\%). These changes were abolished by IPC. After I-R the ICAM-1-specific tissue staining was observed focally only, mainly in the periosteal vessels but not in the muscle vasculature, and this change was not influenced by IPC. Conclusion: These data demonstrate that this type of IPC does not induce inflammatory reactions but confers effective protection by preventing the I-R induced leukocyte activation processes in the rat periosteum. The IPC-provided protection is, however, not mediated by ICAM-1 expression changes in this setting.

Supported by research grant OTKA K60752.

\section{O187}

REFINEMENT OF A RAT SURGICAL MODEL OF REFLUX-INDUCED ESOPHAGEAL CARCINOGENESIS BY TECHNICAL INNOVATIONS AND ANIMAL DISTRESS SCORE SYSTEMS

L. Dall'Olmo' ${ }^{1}$, D. Segat ${ }^{2}$, E. Dazzo ${ }^{2}$, MD Baroni², G. Zaninotto²,

E. Ancona ${ }^{2}$

1 Clinica Chirurgica lii, Padova, Italy

2 Padua University, Padua, Italy

Esophageal adenocarcinoma (EA) development is a multistage process, that origins from a precancerous tissue, called Barrett's esophagus (BE), induced by chronic exposure of esophageal mucosa to duodenogastro-esophageal reflux (DGER). Surgically induced DGER reproduces in experimental animals the steps from normal mucosa to BE, dysplasia and EA. The aim of this work was to reproduce the surgical rat model of DGER-induced BE/EA of Kumagai et al (Scand J Gastroenterol 2003(7):687-691). Three technical improvements were introduced: (1)no pre-operative restriction of food or water intake, (2)left gastric and left inferior phrenic arteries ligation and (3)running suture to perfom intestinal anastomosis. In addition, we applied 2 score sheet systems (Morton DB. ILAR J. 2000;41(2):80-6) to assess pain, distress and discomfort postsurgery. 25 adult male inbred Wistar rats were surgically treated, under inhalation anesthesia with Isoflurane, to induce DGER with a $1,5 \mathrm{~cm}$ side-by-side intestinal anastomosis between the first digiunal loop and the gastro-esophageal junction. The animals were monitored daily (1st month), then weekly, to follow up their clinical conditions and to consider therapeutic administrations. Animals were weighed and scored, using numerical (NSS) and binary score sheet (BSS) systems. The former one 
assigns a numerical score to physiological and behavioural parameters (body weight, appearance, clinical signs, spontaneous and provoked behaviour). BSS system considers non-parametric binary signs.

Two animals died within 24 hours after surgery, for surgical or anesthesia complications and one died at 7th post-operative day (POD) because of an anastomotic fistula. Others were sacrificed. Animal weight was a major parameter of welfare during the follow up and decreased up to $74.1 \% \pm 9.1$ than the pre-operative one, being day 8 the median POD (range: 5-19) of minimum weight. In the 2 nd week most of the animals showed improved clinical conditions and reached their pre-operative weight (PW) at a median POD of 26. Only 4 animals never reached their initial PW and this correlated with a critical follow up (despite of frequent therapies) and pneumonia diagnosis. They were prematurely euthanised around POD 100 (range: 70-120), autoptic evaluation confirming pneumonia (presumably ab-ingestis). Consistently, the main long-term complication of this surgical procedure was clinically evident pneumonia (10 out of 22 cases), correlated to body weight loss, starey coat, nasal discharge and abnormal breathing. 4 animals were unresponsivess to antibiotics and were therefore sacrificed, whereas the others clinically recovered. Finally, one animal was sacrificed (POD 55) because of severe labyrinthitis. The present work allowed us to refine the surgical procedure and post-operative rat care management by the use of a modified technique and application of score sheet systems. Significantly, we obtained an $88 \%$ long-term (>10 weeks) survival rate and ensured timely euthanasia of suffering animals. Preliminary data showed the development of Barrett's esophagus already at 10 weeks after surgery. Therefore, animal sacrifices were planned at 5, 10, 20, 30, 40 and 50 weeks to monitor $\mathrm{BE}, \mathrm{NiN}$ or EA development. Our system appears to be fully suitable to plan in vivo experiments devoted to study BE - NIN EA neoplastic evolution at histological, cellular and molecular level.

\section{8}

\section{TOTAL NITRIC OXIDE AND CATALASE ACTIVITY IN INTESTINAL ISCHEMIA/REPERFUSION INJURY IN RATS}

E. Margaritis ${ }^{1}$, N. Liarakos ${ }^{1}$, A. Pantopoulou², M. Poulakou², D. Mantas¹, M. Kostakis ${ }^{1}$, A. Papachristodoulou ${ }^{1}$, D. Perrea ${ }^{2}$, A. Kostakis

1 Faculty of Medicine, Athens University, Athens, Greece

2 Experimental Labotatory, Medical University, Athens, Greece

Introduction: Intestinal ischemia- reperfusion (IIR) is an often-clinical problem and the oxidative stress plays a significant role in injuries that are created.

Aim: The purpose of this study was to count the oxidative stress by measuring Total Nitric Oxide and Catalase Activity in the blood serum of Wistar rats in which we caused surgical IIR.

Methods: 40 Wistar rats (250-300gr) were separated and randomized in five groups ( $A$ to $E, n=8$ ). In group $A$ (control), after a midline abdominal incision, blood sample was received and the animals were put to death. In the rest of groups the superior mesenteric artery was isolated and occluded for 60 minutes using a non traumatic micro vascular clamp (bulldog). In group B blood and tissue was received and the rats were killed the moment that the clamp was removed, in group $C$ the same procedure took place after 1 hour of reperfusion, in group $D$ after 8 hours of reperfusion and in group $E$ after 24 hours of reperfusion (D, $\mathrm{E}$ groups after undergone a new surgical treatment). Total Nitric Oxide $(\mu \mathrm{mol} / \mathrm{l})$ and Catalase Activity $(\mathrm{nmol} / \mathrm{min} / \mathrm{ml})$ were counted in the blood serum.

Results: Nitric oxide increased as the time of reperfusion reached 8 hours (groups $A=43, B=59 C=85 D=94 \mu \mathrm{mol} / \mathrm{l}$ ) and in 24 hours of reperfusion there was a small reduction $(E=90 \mu \mathrm{mol} / \mathrm{l})$. Catalase activity had the same behaviour (groups $A=33, B=41, C=65, D=151 \mathrm{nmol} / \mathrm{min} / \mathrm{ml}$ ) except that in 24 hours of reperfusion the reduction was bigger $(E=42$ $\mathrm{nmol} / \mathrm{min} / \mathrm{ml}$ ). A statistical significant increment observed in groups $\mathrm{B}$ (0h of reperfusion) and $\mathrm{D}$ (8h of reperfusion) in both substances and a statistical significant reduction in catalase activity between group $C(1 \mathrm{~h}$ of reperfusion) and $E(24 h$ of reperfusion) ( $p<0,05$ respectively). Conclusion: It is clear that the oxidative stress measured by catalase activity and total nitric oxide in blood serum increased after 8 hours of reperfusion. Further more, until the reperfusion reached 24 hours the nitric oxide was still present as the catalase activity dropped to its initial levels (0h of reperfusion) showing that although oxidant procedure decreased $\mathrm{NO}$ levels its remained high in blood. This project is co-financed within Op. Education by the ESF (European Social Fund) and National Resources. EPEAEK II, Project 'PYTHAGORAS II'
O189

\section{INFLUENCE OF HEME OXYGENASE-1 ON MICROCIRCULATION} AFTER ISOGENIC KIDNEY TRANSPLANTATION

\author{
J.P. Hölzen ${ }^{1}$, R. Bahde ${ }^{1}$, Ch. August ${ }^{2}$, E. Minin'², H.U. Spiegel ${ }^{1}$ \\ 1 Surgical Research, Muenster, Germany \\ 2 Gerhard-domagk-institute Of Pathology, Muenster, Germany
}

Background: Microcirculatory disorders are a major part of ischemiareperfusion injury. The imbalance between vasodilators and vasoconstrictors leads to microcirculatory disturbances. Heme oxygenase-1 (HO-1) plays an important role for ischemia-reperfusion injury. The aim of this study was to investigate the impact of heme oxygenase-1 on the microcirculation and on the ischemia-reperfusion injury in an isogenic kidney transplantation rat model.

Methods: 70 male Lewis rats were distributed into three groups. In group 1, exclusively the kidneys were mobilized. In groups 2 and 3, bilateral nephrectomy was performed, and a kidney was orthotopically transplanted on the left side. The donor animals in group 3 additionally received preconditioning with the $\mathrm{HO}-1$ inductor hemin. 24 hours after reperfusion graft function and morphology were examined.

We examined the HO-1 expression as well as the endothelial and inducible nitric oxide synthase (eNOS and iNOS) by means of immunohistochemistry with a semiquantitative score. Microcirculation was investigated by in-vivo microscopy of the renal surface one hour after reperfusion.

Results: Microcirculation was improved by a significant enlargement of the vascular diameter and of the capillary flow. HO-1 preconditioning led to significantly lower serum creatinine and serum urea, less histological damage and iNOS expression. The differences between the transplanted animals were not significant in eNOS expression.

Conclusion: Treatment with hemin improves microcirculation by induction of $\mathrm{HO}-1$ and reduces ischemia-reperfusion injury after kidney transplantion. A reduced expression of iNOS shows the cytoprotective effect of HO-1. Because of the unchanged eNOS expression in both groups the vasodilatory effect of hemin preconditioning may be rather due to heme oxygenase-1 but not to NO.

O190

SURGICAL TRAUMA INDUCES ELEVATED H2O2- AND LPOLEVELS, DETECTABLE IN BLOODPLASMA AND PERITONEAL LAVAGE FLUID IN RATS

J.B.C. van der Wal, J. Nieuwenhuizen, W. Sluiter, J. Jeekel, J.F. Lange Erasmus University Medical Center Rotterdam, Rotterdam, The Netherlands

Objective: To determine whether surgical trauma causes detectable changes in levels of reactive oxygen species (ROS) in vivo.

Background: Polymorphonuclear cells (PMN) play an important role in the healing process after damage of the peritoneum. Indirect evidence shows that ROS produced by PMN are another sequela of the inflammatory reaction caused by surgical trauma. Besides beneficial effects, such as destruction of invading micro-organisms and degradation of damaged tissue, the oxidative potential can result in additional (peritoneal) tissue destruction. The amount of peritoneal damage is positively correlated to post-operative adhesion formation as well as to local tumor recurrence, and various studies have shown that administrating ROS scavengers leads to less adhesion-formation and less tumor recurrence. Surprisingly, the actual levels of the various ROS in vivo have never been reported to exclude the possibility that the beneficial effect of ROS scavengers is an intrinsic effect of these reagents.

Methods: According to our previously optimised adhesion-model, 7 animals (female Wag/Rij rats) were operated. In brief: under isoflurane anaesthesia and aseptic conditions a laparotomy was performed using a midline incision of $5 \mathrm{~cm}$. A small oval, $2 \mathrm{~cm}$ in length and $0.5 \mathrm{~cm}$ in width, was then excised on both lateral sides of the parietal peritoneum, simulating surgical trauma, after which the abdomen was closed in two layers. After 5, 24 and 48 hours, peritoneal lavage was performed and blood samples were obtained. Lavage fluid and blood samples of 3 nonoperated animals were used as baseline values. The chain-breaking antioxidant butylated hydroxytoluene was added to the cell-free lavage fluid and the plasma in order to prevent any artificial increase in the levels of lipid peroxides. The levels of hydrogen peroxide and lipid peroxides were determined spectrophotometrically using the ferric-sensitive dye xylenol orange by taken advantage of the ability of these ROS 
to selectively oxidize ferrous ions (FOX2 assay). After determination of these baselines, animals were operated according to the same model. Animals were randomized to receive scavengers postoperatively, or as control. Animals were sacrificed 5,12 and 24 hours postoperatively and $\mathrm{H} 2 \mathrm{O} 2$ and LPO levels were determined in plasma and lavage fluid.

Results: Baseline-values of $\mathrm{H} 2 \mathrm{O} 2$ in non-operated animals in lavage fluid and plasma were $1.091 \pm 0.991 \mathrm{nmol} / /$ en $1.258 \pm 1.332 \mathrm{nmol} / \mathrm{l}$ respectively; baseline-values of LPO in lavage fluid and plasma were $2.199 \pm 2.520 \mathrm{nmol} / \mathrm{l}$ and $1.022 \pm 1.440 \mathrm{nmol} / \mathrm{l}$, respectively. Five hours postoperatively the level of $\mathrm{H} 2 \mathrm{O} 2$ rose to a maximum of $3.109 \mathrm{nmol} / \mathrm{l}$ en $3.041 \mathrm{nmol} / \mathrm{l}$, in lavage fluid and plasma respectively. Animals which had received scavengers showed decreased levels compared to baseline $(0.351 \pm 0.402 \mathrm{nmol} / \mathrm{l}$ en $0.254 \pm 0.439 \mathrm{nmol} / \mathrm{l}$ in lavage fluid and plasma respectively). The levels of LPO showed no change after 5 hours; only after 12 hours a small increase was seen in the lavage fluid. After 24 hours all levels were returned to baseline-value.

Conclusion: This experiment shows that it is possible to measure levels of ROS in plasma and lavage fluid. After surgical trauma these levels increase and this increase is avoidable by adminstration of ROSscavengers.

\section{O191}

POSSIBLE EFFECTS OF PACAP ON THE ACTIVATION OF MITOGEN ACTIVATED PROTEIN KINASES FOLLOWING WARM ISCHEMIA/REPERFUSION IN BOWEL TISSUE

A. Ferencz, A. Ferencz, B. Racz, B. Cserepes, M. Kürthy, G. Jancso, A. Lubics, K. Kalmar-Nagy, D. Reglodi, E. Roth University of Pecs Medical School, PECS, Hungary

Introduction: Warm ischemia/reperfusion (I/R) cause severe tissue oxidative injury on small bowel. Oxidative stress triggers several intracellular pathways via mitogen activated protein (MAP) kinases. MAP kinases include the extracellular signal related kinase (ERK), c-Jun N-terminal kinase (JNK), and p38 MAP kinase. Pituitary adenylate cyclase-activating polypeptide (PACAP) is present and plays a central role in the intestinal physiology. We aimed to investigate the effect of PACAP on the activation of MAP kinases during small bowel warm I/R.

Methods: Warm I/R groups and PACAP-treated groups were designed on Wistar rats. Group (G) I: sham operated, no-ischemia; GII: 1 hour ischemia; GIII: 2 hours ischemia; GIV: 3 hours ischemia; GV: sham operated, no-ischemia+PACAP; GVI: 1 hour ischemia+PACAP; GVII: 2 hours ischemia+PACAP; GVIII: 3 hours ischemia+PACAP. Reperfusion started at the end if ischemia, and lasted for 3 hours in all groups. In GV-GVIII 5 ug single bolus and 25 ug continuous PACAP were administrated during reperfusion intravenously. Small bowel biopsies were collected after laparotomy, at the end of ischemia and reperfusion periods. In small bowel tissue the activation of MAP kinases (ERK1/2, JNK1/2, p38 MAPK) were investigated by immunocytochemistry and Western blot analysis.

Results: Our results showed that in warm ischemia groups (GII-GIV) the phosphorilated ERK1/2 level decreased, meanwhile the phosphorilated JNK1/2 and p38 MAP kinase activation elevated compare to control level. In all treated groups (GVI-GVIII) PACAP enhanced the phosphoERK1/2 appearance and decreased JNK and p38 MAP kinase activity by the end of reperfusion periods.

Conclusion: We have provided evidence that warm I/R decreased phosphorilated ERK1/2 level and increased JNK1/2 and p38 MAP kinase activity. Which means, that warm I/R caused a trigger effect to apoptotic cell death. In contrast, PACAP treatment induced the protective signalling pathways against oxidative injury by MAP kinases in bowel tissue. Supported by Grants OTKA F046593 and OTKA T046589.

\section{O192}

OBESITY \& SMOKING INFLUENCE POST-OPERATIVE INFLAMMATORY RESPONSE

S. Irukulla1 , J. John¹, P. Collinson², R. Bhate'1, D. Gage ${ }^{2}$, A. Abulafi',

A. Mendall ${ }^{1}$

1 Mayday University Hospital, Croydon, United Kingdom

2 St.georges Hospital, London, United Kingdom

Background: Obesity \& smoking are associated with increased basal inflammation. Prevalence of obesity alarmingly increasing.Obesity has been strongly associated with Metabolic syndrome.Surgery in obese is associated with greater morbidity. No previous studies have evaluated inflammatory response in obese comparing with normal weight patients after a standardized surgery.

Objectives: Primary: To evaluate inflammatory response in obese following surgery measured by raise in CRP at 24 hours. Secondary : To demonstrate the influence of environmental factors on inflammatory response.

Methodology: Non-randomized observational study. Inclusion criteria: 1.Primary,2. unilateral inguinal hernia,3. male patients Exclusion Criteria: 1.Bilateral 2. recurrent hernia, 3. Unfit for GA, 4. Uncontrolled diabetes,5. Organ failure, 6 . Existing inflammatory or infectious process. Study period: Since March 2006 . Measurements: $\mathrm{W} ; \mathrm{H}$ ratio and BMI Blood samples: were collected before surgery then $6,24,48 \mathrm{hrs}$ and 7 days after surgery. Patients were discharched on the day of surgery when appropriate. Main researcher visited patients at their home for collection of postoperative samples.

Results: Statistical analysis using Stratview software system. Total of 65 patients. All received GA. Age range $20-90$ (median $59 \mathrm{yrs}$ ), smoking status (15 current, 21 ex-smokers and 29 non-smokers), physical activities (25-regular exercise) and duration of surgery (range 10-40, median 18 minutes). On univariate analysis we found significant positive correlation between age $(p=0.02)$, BMI $(p=0.046)$ and basal CRP. We noticed a trend between age and CRP raise, though significance was borderline $(p=0.07)$

Basal 24 hours

W;H $\quad p=0.047 p=0.0031$

Smoking $p=0.016 \quad p=0.034$

Results are controlled for age, smoking, exercise, duration of surgery \& wound length

Conclusion: Central obesity $(\mathrm{W} ; \mathrm{H})$ and smoking appear to modulate postoperative inflammatory response. This inappropriate inflammatory response could explain increasing postoperative morbidity following more major surgeries.

\section{3}

CRITICAL ROLE OF P-SELECTIN AND PSGL-1 IN RADIATIONINDUCED PLATELET-ENDOTHELIUM INTERACTIONS IN THE COLON

I.A Mihaescu, C. Thornberg, S. Mattsson, B. Jeppsson, H. Thorlacius Lund University, UMAS, Malmø, Sweden

Background: Adjuvant radiation is frequently used in the treatment of different types of tumours although associated with serious side effects. Platelets may be involved in radiation-induced enteritis. The aim of our study was to define the adhesive mechanisms behind radiotherapy-induced platelet recruitment in the colon.

Method: All mice, except control animals, were exposed abdominal radiation with a single dose of 20 Gray. Mice were pretreated with an isotype-matched control antibody, a monoclonal antibody directed against P-selectin and against P-selectin glycoprotein ligand-1 (PSGL1). Platelets rolling and firm adhesion in the colon were determined by use of inverted intravital fluorescence microscopy $16 \mathrm{~h}$ after radiation. Results: Imunoneutralization of P-selectin reduced platelet rolling by $60 \%$ and adhesion by $67 \%$ and inhibition of PSGL- 1 reduced platelet rolling and adhesion by $85 \%$ and respectively $82 \%$, in radiated mice. Systemic platelet counts showed no significant difference between the experimental groups.

Conclusion: This study demonstrates that platelet rolling is mediated by P-selectin and PSGL-1 and that inhibition of P-selectin and PSGL-1 decrease firm platelet adhesion in radiation-induced enteritis. Thus, we conclude that P-selectin and PSGL-1 may be useful targets in order to protect against pathological inflammation in the colon associated with radiotherapy

\section{O194}

EXPERIENCE WITH CORTICAL TUNNEL FIXATION IN ENDOSCOPIC

\section{BROW LIFT}

A. Abood ${ }^{1}$, C.M. Malata ${ }^{2}$

1 University College Hospital, London, United Kingdom

2 Addenbrooke's University Hospital, Cambridge, United Kingdom

Background: Endoscopic brow lift1,2 has become a popular method world-wide for rejuvenation of the upper third of the face. Controversy, however, remains over the optimum technique for the fixation of the forehead and brow3. This paper presents a single surgeon's experience with the endogenous technique of paramedian cortical tunnel fixation4 in patients undergoing endoscopic brow lifts. 
Patients and methods: This is a retrospective study of all patients that had undergone endoscopic brow lift by the senior author over a three year period. Brow position was maintained with 2/0 polypropylene (prolene) sutures anchored through outer table paramedian cortical bone tunnels. Temporal fixation of the temporoparietal fascia to the deep temporal fascia was achieved with the same suture material.

Results: Over the four year period, 29 patients had an endoscopic brow lift performed for aesthetic and functional reasons. All cases were bilateral. Twenty-five patients ( $86 \%)$ were female and $4(14 \%)$ were male. The mean age was 59 years (range: $32-82$ years). Patient follow-up ranged from 3 months to 36 months (mean: 18 months). One third of patients $(n=10)$, had another aesthetic procedure carried out at the same time as the brow lift (facelift, $n=3$, Upper lid Blepheroplasty, $n=7$ ). There were no early postoperative problems of bleeding, facial nerve paralysis or infection. One patient had a paramedian fixation suture removed under local anaesthetic (LA), 6 months later because of a palpable suture end, necessitating subsequent knot burying in the tunnel in subsequent cases. Another had a fixation suture removed under LA six weeks postoperatively for a non-specific pressure sensation. A third patient developed troublesome forehead/scalp dysaesthesiae. Notably, there were no cases of alopecia at the incision/ fixation sites, relapses of brow ptosis, or troublesome scalp itching. There was no intraoperative conversion to open/coronal brow lifting

Discussion and conclusion: Cortical-tunnel suture fixation provided simple, stable, and reproducible method of maintaining brow position in endoscopically assisted forehead rejuvenation with low morbidity. A modification of the technique designed to prevent exposure of the suture ends or knots will be illustrated.

References:

1.Isse NG. Endoscopic facial rejuvenation: endoforehead, the functional lift. Case reports. Aesthetic Plast Surg. 1994 Winter;18(1):21-9. 2. Core GB, Vasconez LO, Graham HD 3rd.Endoscopic browlift. Clin Plast Surg. 1995 Oct;22(4):619-31.

3.Rohrich RJ, Beran SJ. Evolving fixation methods in endoscopically assisted forehead rejuvenation: Controversies and rationale. Plast Reconstr Surg 1997; 100: 1575.

4. Mckinney $\mathrm{P}$, Celleti S, Sweis I. An accurate technique for fixation in endoscopic brow lift Plast Reconstr Surg 1996; 97(4): 824'7.

\section{O195}

USING 3D IMAGING TECHNOLOGY TO PLAN RECONSTRUCTION OF ASYMMETRIC FACES

\section{A.J. Ponniah1, H. Witherow², R. Richards ${ }^{3}$, C. Ruff ${ }^{3}$, R. Evans ${ }^{2}$, D. Dunaway ${ }^{2}$ \\ 1 Addenbrookes Hospital, Cambridge, United Kingdom \\ 2 Great Ormond Street Hospital, London, United Kingdom \\ 3 University College London, London, United Kingdom}

Background and aims: Facial asymmetry poses a difficult problem to the reconstructive surgeon. As many of the patients do not have a definable midline, planning surgery is difficult. This research aims to produce virtual images of potential end points of surgery to guide the surgeon in the planning process. Cutting edge technology was utilized taking advantage of recent advances in 3D imaging technology and mathematical modelling.

Methods: Virtual images were produced by manipulating 3D CT images of patients using geometric morphometrics. A mathematical model of normal variation was created by landmarking 3D CT scans of dry juvenile skulls obtained from US and UK collections. The landmarked data was analysed using Kendall's space shape and principal component analysis. Patients with facial asymmetries due to hemifacial microsomia, Parry-Romberg syndrome and radiation damage were analysed using the same techniques. The mathematical model created was used to cre ate a virtual image of the patient which could be warped by the surgeon to the desired position.

Results: 51 landmarks were used to define the shape of the facial skeleton. The landmarks were tested for repeatability. 49 of the landmarks lay within the $95 \%$ confidence interval that they would be within $2 \mathrm{~mm}$ of the target. Using principal component analysis, normal variation and variation within an asymmetric population were analysed. Using the landmarks of 10 skulls from the normal population aged 3 to 18 , a point distribution model was created. Over $99.9 \%$ of variation within the population could be captured within the first 9 modes of variation. It was found that the first 3 modes of variation accounted for $82.9 \%$ of the population. The whole data set (40 skulls) was used to create a virtual image where only the parts of the skull amenable to surgery moved and the rest of it remained unchanged. The process also enabled movement towards more 'normal' images. The creation of a user interface will allow surgeons to manipulate images till the desired virtual result is achieved. The virtual image can then aid planning the surgical procedure

Summary and conclusion: Creating a virtual image of a potential endpoint of surgery is especially difficult in patients with facial asymmetry as there is no definable midline. Using geometric morphometric tools and techniques a significant step towards a potential solution has been achieved. The virtual image can be used to plan craniofacial surgery or can be the basis of prosthetic implants which can improve the bony contour.

\section{O196}

\section{INFLUENCE OF NEOADJUVANT CHEMOTHERAPY ON OUTCOMES} OF IMMEDIATE BREAST RECONSTRUCTION: A RETROSPECTIVE COMPARISON

A. Ismail ${ }^{1}$, K. Azzawi ${ }^{2}, \mathrm{H}$. Earl $^{2}$, C. Malata ${ }^{2}$

1 Addenbrooke's Hospital, Cambridge, United Kingdom

Introduction: Immediate breast reconstruction following neoadjuvant chemotherapy is relatively new and raises concerns about increased peri-operative complications. Moreover, it has the potential to delay planned adjuvant radiotherapy. This study examined the effect of neoadjuvant chemotherapy on reconstructive outcomes and the commencement of adjuvant (post-operative) radiotherapy.

Methods: A retrospective review of a single surgeon's immediate breast reconstructions (IBRs) performed from 2000 to 2006 was undertaken. The recipients of neoadjuvant chemotherapy were compared with non-recipients (controls). Statistical analysis was carried out using the unpaired Student's 't' test for the mean ages and the Fisher's exact test for the reconstructive outcomes. The study was confined to patients operated on by a single surgeon in order to eliminate inter-operator variability.

Results: Over the seven-year period a total of 133 IBRs were performed in 117 patients. These comprised 39 free tissue transfers, 55 pedicled flaps (46 latissimus dorsis and 9 of the transverse rectus abdominis myocutaneous type), and 39 implant-onlys. The reconstruction types in both groups were broadly comparable.

Twenty-five patients (21\%; 27 reconstructions), mean age 46 years $(r=32-60)$, had neoadjuvant chemotherapy prior to mastectomy and reconstruction ( $93 \%$ with flaps). The failed reconstruction rate was $4 \%(1 / 27)$. The re-operation rate for major complications including flap failure and severe peri-implant capsular contracture was $11 \%(3 / 27)$. The incidence of minor complications such as delayed healing was $22 \%(6 / 27)$.

The control group consisted of 92 patients (106 reconstructions; $65 \%$ with flaps) with a mean age of 51 years $(r=33-69)$, making them significantly older $(p=0.01)$. The failed reconstructions were $3 \%(3 / 106)$, re-operations $11 \%(12 / 106)$, and minor complications $9 \%(9 / 106)$. These outcomes were not significantly different from the neoadjuvant chemotherapy group (Fisher's exact test: $p$ values $=1.63,0.58$ and 0.24 respectively). Radiotherapy was delayed in $8 \%(2 / 25)$ of chemotherapy recipients versus $9 \%(2 / 22)$ of controls $(p=0.85)$

Conclusion: In our series, neoadjuvant chemotherapy did not appear to increase the risk of major surgical complications following mastectomy and immediate breast reconstruction or inordinately delay the institution of adjuvant (post-operative) radiotherapy. As expected, the patients receiving neoadjuvant chemotherapy prior to mastectomy and reconstruction were significantly younger than the controls. Our data suggest a higher incidence of minor complications in the neoadjuvant group which, although clinically important, did not attain statistical significance.

\section{O197}

AN IN-VIVO ANATOMICAL STUDY OF HUMAN PREPUCE SHOWED DIFFERENT PATTERN OF BLOOD SUPPLY OF THE FRAENULAR ARTERY

G. Virich, A. Ismail, K.H. Azzawi

1 Addenbrooke's Hospital, Cambridge, United Kingdom

An in-vivo anatomical study of human prepuce showed different pattern of blood supply of the fraenular artery

Background: Circumcision is one of the most popular surgical proce- 
dures. The injury of the fraenulum artery during circumcision and the possible ischemia was accused as responsible of meatal stenosis based on stastical study.

Aim: The authors present an in-vivo clinical anatomical study. This is to investigate the blood supply pattern of the prepuce, on the ventral side. Methods: In one year period (1995), 19 male children had circumcision. All the children were circumcised in surgical theatre settings in a university hospital. The procedure was performed without anaesthetic with agreement of parents; as it was the usual standard practice. In cases of fraenula brave, the procedure included cutting the fraenulum with its artery and checking the source of bleed on either end (distally and proximally).

Results: the study showed that contrary to the previous general concept; the fraenulum artery comes from distal to proximal when the prepuce is in retracted position. None of the patient had any post-operative haemorrhage or meatal stenosis. This is contrary to the well known blood supply pattern of prepuce, which was explained before by Taylor. Conclusion: This would explain an anatomical argument of the possible cause of meatal stenosis after circumcision. Moreover, it would provide an anatomical tip to help in reducing the risk of haemorrhage during and after the procedure. Actually, bleeding could be reduced by controlling the fraenular artery from its distal end, early in the procedure (when needed)

\section{8}

\section{ANGIOGENIC INTEGRATION OF BIO-ARTIFICIAL MATRICES IN AN EXPERIMENTAL MODEL}

M. E. Amon', Y.H. Harder², B. B. Bucsky³, H.G.M. Machens ${ }^{3}$, M.D. Menger ${ }^{2}$

1 University Of Saarland, Homburg/Saar, Germany

2 Institute For Clinical \& Experimental Surgery, Homburg/Saar, Germany 3 Clinic For Plastic And Hand Surgery, Lubeck, Germany

Introduction: Coverage of extensive defect wounds after severe trauma or burn injuries still represents a challenge for reconstructive surgeons. The prevention of contractures due to distinct scarring by the use of bio-artificial matrices, combined with delayed transplantation of skin grafts, is common in clinical practice. However, little is known about the angiogenic reaction of the underlying tissue on transplantation of the matrices. In the present study, we established an experimental model for analysis of angiogenesis and inflammatory response of three different, routinely used matrices.

Methods: In C57BL/6-mice, a chronical dorsal skinfold chamber was implanted. After 2 days, pieces $(2 \times 2 \mathrm{~mm})$ of the different matrices (Integra, Alloderm, Matriderm) were implanted into the observation windows of the chambers. By means of intravital fluorescence microscopy, the number of angiogenesis-positive observation fields and the microvessel density was analysed in the central and border zones of the transplant. For determination of biocompatibility, venular leukozyte adherence was evaluated in host vessels at the border zone.

Results: Already 6 days after implantation of Integra, $30 \%$ of the peripheral observation fields were positive for angiogenesis, in Matriderm only $17 \%$ showed signs for blood vessel formation. In contrast, Alloderm showed no signs of angiogenic reaction. At the end of the experiments at day 14 , there was angiogenesis in nearly all peripheral observation fields in the Integra- and Matriderm groups ( $88 \pm 11 \%$; $89 \pm 5 \%)$, whereas the transplantation of Alloderm resulted in a significantly blunted reaction with only $42 \pm 20 \%$ of angiogenesis-positive fields. The density of newly formed blood vessels was markedly higher after implantation of Integra $(144 \pm 40 \mathrm{~cm} / \mathrm{cm} 2)$ and Matriderm $(151 \pm 36 \mathrm{~cm} / \mathrm{cm} 2)$ as compared to the Alloderm-group $(93 \pm 50 \mathrm{~cm} / \mathrm{cm} 2)$. However, in no animal we could observe angiogenesis in the central part of the transplanted matrices during the course of the experiments. Dilation of the venular vessels was observed after transplantation of Alloderm $(52 \pm 13 \mathrm{~mm})$, but not after Integra $(25 \pm 5 \mathrm{~mm})$ or Matriderm $(22 \pm 2 \mathrm{~mm})$. A significant increase in venular leukocyte adherence as a sign of forign-body-reaction did not occure in any of the animals.

Discussion: With the present study, we could demonstrate for the first time, that the model of the dorsal skinfold chamber in mice ideally serves for repetitive analysis of angiogenesis in bio-artificial matrices. Our results demonstrate that both Matriderm and especially Integra exhibit a markedly superior angio-integration as compared to Alloderm. Further studies using this model will provide evidence if the supplementation of growth factors enabels a more rapid and more pronounced angiogenic reaction and thus may improve the take-rate of transplants.
O199

SIMULTANEOUS VERSUS SEQUENTIAL CONTRALATERAL BALANCING BREAST SURGERY IN POSTMASTECTOMY BREAST RECONSTRUCTION: A RETROSPECTIVE COMPARISON

\author{
D. Kumiponjera, R. Seaward, H. Kamboj, M. Malata \\ Addenbrooke's Hospital, Cambridge, United Kingdom
}

Introduction: Contralateral balancing breast surgery is an important part of postmastectomy breast reconstruction since the achievement of symmetry is its ultimate goal. Such symmetrization procedures can be performed either at the time of the reconstruction (simultaneous) or later (delayed / sequential). The ideal time for performing contralateral surgery remains controversial. This study therefore aims to determine whether the timing of balancing breast surgery influences the outcome with respect to the number and nature of subsequent revisional procedures required to achieve symmetry after simultaneous or sequential surgery.

Patients and methods: All patients who had breast reconstruction undertaken by the senior author (CMM) between 1999 and 2006 were retrospectively reviewed. Details of patients undergoing contralateral balancing breast surgery were collected. The data were analysed in terms of breast reconstruction types, balancing procedure, nature and number of subsequent revisional procedures needed to achieve satisfactory symmetry.

Results: Over the seven year period, 203 patients (mean age $=50$, range $=29-70$ ) underwent immediate $(125)$ and delayed (78) breast reconstructions. The reconstructions comprised 48\% (97) abdominal flaps, 37\%( 75 ) latissimus dorsi flaps and 15\%(31) implant only reconstructions. Two thirds of the patients (114) had balancing surgery ( $79 \%$ simultaneous and $21 \%$ sequential). The balancing procedures consisted of 38 breast reductions, 34 mastopexies, 22 augmentations and 30 mastectomies and implants or abdominal flap reconstruction. The revision procedures included liposuction or open surgical resection of the reconstruction, implant exchange/adjustment and mastopexy. The revision rate was $13 \%(12 / 90)$ in the simultaneous group and $16 \%$ $(4 / 24)$ in the sequential group. This did not reach statistical significance $(p=0.9) \times 2$ test.

Conclusion: Simultaneous contralateral balancing breast surgery does not lead to a higher revision rate contrary to expectation. It should therefore not be denied to patients requesting it on account of the putative difficulty in achieving symmetry

\section{O200}

MORPHOLOGIC FEATURES IN THE REGENERATING LIVER A COMPARATIVE INTRAVITAL-, LIGHTMICROSCOPICAL AND ULTRASTRUCTURAL ANALYSIS WITH FOCUS ON HEPATIC STELLATE CELLS

\section{T. Budny}

Muenster, Germany

Introduction: Different cell types play a role in the liver regeneration. The present study reveals morphological key steps of rat liver regeneration by means of intravital microscopy (IVM) in correspondence to light and electron microscopy as well as immunohistochemistry focusing on hepatic stellate cells (HSC).

Materials and methods: In 49 Lewis rats liver regeneration was induced by a $2 / 3$ hepatectomy. Animals ( $n=7$ each) were sacrificed after $0,1,2,3,4,7$ and 14 days. Morphological features of the regenerating rat liver were investigated by light microscopy regarding the histological criteria of cell swelling, mitosis, immigration of sinusoidal cells into hepatic clusters and endothelium structure, immunohistochemistry (SMA, Desmin, VEGF, VEGFR), intravital microscopy with respect to hepatic cord width, sinusoid density, number of hepatocytes and HSC and transmission electron microscopy focussed on cell-cell interactions.

Results: IVM showed the number of HSC and hepatocytes were maximum reduced at the 3 rd day and begin to reincrease up to normal values at day 7 . Angiogenesis started on the 4th day noticeable as an increase of the sinusoid density and hepatocyte clusters surrounded by endothelial cells. Expression of VEGF and VEGF receptor showed a strong increase on the 3rd, 4th and 7th with parallel expression of SMA, a marker of HSC activation. Furthermore the cell-cell contact between hepatocyte and HSC were on this days increased visible in electron microscopy. An increase of the apoptotic index was only recorded on the 7 th postoperative day for the non-parenchymal cells. 
Conclusion: IVM represents an appropiate method to chronologically investigate the liver regeneration in vivo, particularly HSC due to their autofluorescence. Activated HSCs are involved in the angiogenesis during liver regeneration probably by increasing the expression of VEGF. Apoptosis is probably one pathway for deactivation of HSCs.

\section{O201}

\section{THE EFFECT OF DIPYRIDAMOLE ON LIVER REGENERATION IN EXPERIMENTALLY HEPATECTOMIZED RATS}

T. Artis' ${ }^{1}$, H.E. Esin ${ }^{2}$, A.A. Akcan ${ }^{1}$, F.M. Mutlu1, S.A. Artis ${ }^{1}$, Z.Y. Yilmaz 1 Erciyes University Medical School, Kayseri, Turkey

Background and aim: The phosphodiesterase inhibitors (PDEIs) have been proposed to improve hepatic reperfusion injury and hepatosplanchnic circulation, but the effects of these agents on liver regeneration have not been investigated thoroughly. To assess the effect of dipyridamole, a type $5 \mathrm{PDEI}$, on liver regeneration in experimentally hepatectomized rats.

Materials and methods: This experimental study has been performed in Hakan Cetinsaya Experimental and Clinical Research Center (DEKAM) at Erciyes University Medical Faculty between April and June 2006. Sixty Wistar-Albino rats weighting between 250 and $350 \mathrm{~g}$ were used in the study. Rats were divided into two as the control and dipyridamole groups. Each group has been divided into three subgroups containing 10 rats each. First, jugular venous catheters were placed before hepatic resection. After $70 \%$ liver resection has been performed to the rats, upon resection dipyridamole infusion to the study group and $0.9 \% \mathrm{NaCl}$ infusion to the control group has been done. After $70 \%$ liver resection the study and control groups received dipyridamole and 0.9 $\% \mathrm{NaCl}$ infusion, respectively. Then the resected wet liver tissues were weighted. Rats were allowed to survive for 24th, 48th and 72 nd hours and then they were sacrificed. Blood samples were collected from vena cava inferior and remaining liver tissues were resected and weighted. Serum AST, ALT, ALP, albumin and PT were measured. Relative liver weight was used as a morphological parameter for liver regeneration. Histopathologic assessment has been performed to determine mitotic index and Proliferating Cellular Nuclear Antigen (PCNA).

Results: There were statistically significant differences between dipyridamole and control groups in ALP and relative liver weights at 24th, 48th and 72 nd hours $(p<0.05)$. There were also statistically significant differences between the groups in PT and albumin levels at all times but 48th hours for PT and 24th and 72th hour for albumin levels $(p<0.05)$. Mitotic index and PCNA labelling index were significantly higher in dipyridamole group for each time period $(p<0.05)$.

Conclusion: Dipyridamole increases liver regeneration both morphologically and functionally in experimental liver resection model in rats. With further studies, clinical implementation should be considered.

\section{$\mathrm{O} 202$}

GLYCEMIC CONTROL REGULATES ARGININE AND ADMA LEVELS BY PRESERVING DDAH-ACTIVITY DURING CRITICAL ILLNESS

M.C. Richir ${ }^{1}$, B. Ellger ${ }^{2}$, L. Langouche ${ }^{2}$, Y. Debaveye ${ }^{2}$, T. Teerlink ${ }^{1}$, I. Vanhorebeek ${ }^{2}$, P.A.M. van Leeuwen ${ }^{1}$, G. van den Berghe ${ }^{2}$

1 VU University Medical Center Amsterdam, Amsterdam,

The Netherlands

2 Catholic University Of Leuven, Leuven, Belgium

Introduction: The endothelium plays a pivotal role in the control of vascular tone by releasing nitric oxide (NO) from arginine, the sole substrate for the enzyme NO-synthase (NOS). Asymmetric dimethylarginine (ADMA), an endogenous derivate of arginine, inhibits NOS and is thereby a determinant of the bio-availability of NO. Critically ill, surgical patients suffer high plasma levels of ADMA which is associated with increased mortality. Tight glycemic control in these patients by intensive insulin therapy affects the endothelium and plasma levels of arginine and ADMA. We studied the relative impact of maintaining normoglycemia and glycemia-independent actions of insulin on plasma and tissue levels of arginine and ADMA, DDAH-activity and organ function.

Methods: In a TPN-fed rabbit-model of prolonged critical illness we assessed the impact of maintaining normoglycemia/normoinsulinemia (NG/NI), normoglycemia/hyperinsulinemia (NG/HI), hyperglycemia/normoinsulinemia $(\mathrm{HG} / \mathrm{NI})$ and hyperglycemia/hyperinsulinemia $(\mathrm{HG} / \mathrm{HI})$ over 7 days on plasma and tissue levels of arginine and ADMA, DDAHactivity in liver, kidney, myocardium and skeletal muscle.
Results: Plasma arginine levels decreased and ADMA levels increased in the hyperglycaemic animals compared to normoglycemic animals $(p<0.01)$. In myocardium and skeletal muscle, arginine concentration of $\mathrm{HI} / \mathrm{HG}$ animals was lower compared to the other groups and healthy controls $(p<0.05)$. In liver, both hyperglycaemic groups revealed lower arginine levels as normoglycemic animals $(p<0.05)$. In myocardium, kidney and liver, ADMA levels were higher in the hyperglycaemic animals compared to normoglycemic animals $(p<0.05)$. In kidney and liver both hyperglycaemic groups revealed lower DDAH activity compared to normoglycemic animals $(\mathrm{p}<0.05)$. The arginine over ADMA ratio correlated with DDAH-activity in kidney $(R 2=0.33 ; p<0.001)$ and liver $(R 2=0.26 ; p<0.001)$. DDAHactivity in liver and kidney correlated with plasma markers of liver function $(R 2=0.55 ; p<0.001)$ and kidney function $(R 2=0.42 ; p<0.001)$.

Conclusion: Hyperglycemia independent of insulin levels in critically ill animals, significantly decreases arginine levels, increases plasma and tissue levels of ADMA and decreases the activity of DDAH in the liver and kidney. Maintaining normoglycemia rather than hyperglycemia maintained physiologic arginine and ADMA levels by preserving enzyme activity of DDAH. Because arginine and ADMA determines NO-bioavailability, this may have serious consequences for the microcirculation in critically ill patients.

\section{O203}

FASTING AND SHORT-TERM CALORIE RESTRICTION PROTECT AGAINST ACUTE OXIDATIVE DAMAGE INDUCED BY ISCHEMIC INJURY IN MICE

M. Verweij, J.R. Mitchell, H.P. Roest, S. van den Engel, J.H.J. Hoeijmakers, J.N.M. IJzermans, R.W.F. de Bruin

Erasmus Medical Center Rotterdam, Rotterdam, The Netherlands

Introduction: Ischemia reperfusion injury (iri) may induce acute renal failure, and is considered a serious complication after trauma, shock and extended surgery, as well as a risk factor for the occurrence of acute rejection and chronic allograft nephropathy after kidney transplantation. The primary mediators of iri are reactive oxygen species (ROS), which are produced during reperfusion of the ischemic kidney. The only known intervention that extends lifespan is calorie restriction (CR). CR is thought to prolong lifespan in part by reducing oxidative damage in the body, which is thought to be the most important reason why we age. $\mathrm{CR}$ reduces the production of ROS in mitochondria, and upregulates antioxidant defence systems.

Aim: To investigate if CR protects against surgically induced acute oxidative damage in mice.

Materials and methods: Liver or renal ischemic injury was induced in male C57BL/6 mice, which were subjected to either 30\% CR for 2-4 weeks or to 24-72 hours of fasting. Renal ischemia was induced by clamping the left kidney for 37 minutes. Partial $(70 \%)$ liver ischemia was induced by clamping the portal triad for 75 minutes. Kidney function (assessed by blood urea nitrogen and serum creatinine) and mortality were measured in renal ischemia experiments, and liver damage (assessed by serum ASAT levels and histology) in liver experiments.

Results: In the ad libitum fed group, $70 \%$ of the animals died within $2-4$ days due to acute renal failure, whereas all animals in the four weeks $30 \%$ CR group survived. Renal function was significantly better in CR animals on days 1-2 than in the surviving ad libitum fed mice. Similar results were observed in mice after two weeks of $30 \%$ CR. Following these remarkable results, we investigated whether fasting was also able to protect against renal iri using the same model. Although even 24 hours of fasting was significantly beneficial for survival $(90 \%$ survival vs. $30 \%$ in the ad libitum fed group), with a maximal benefit reached by 48 hours (100\% survival), kidney function was best in the 72 hours fasted group. We next investigated if these benefits were organ specific, by inducing iri in the liver. Six hours after reperfusion, the 48-72 hours fasted animals were significantly protected from hepatic injury then the ad libitum fed animals. Histological examination 24 hours after reperfusion demonstrated that these livers had significantly less haemorrhagic necrosis.

Conclusion: Two or four weeks of CR protected C57BL/6 mice against a potentially lethal ischemic insult. Similar beneficial effects on animal survival and kidney function were seen after 24-72 hours of water-only fasting. Benefits of fasting were protective against liver ischemia as well. These results suggest that the mechanisms involved in extended longevity may also be tapped for short-term benefits such as ischemia reperfusion injury. 
O204

ROLE OF P38 MITOGEN ACTIVATED PROTEIN KINASE SIGNALING PATHWAY IN REGULATING SEPTIC LUNG INJURY

M. Asaduzzaman, Y.G Wang, T. Henrik

Lund University, Malmo, Sweden

Leukocyte-mediated tissue damage is a key feature in septic lung injury although the signaling mechanisms behind pulmonary recruitment of leukocytes remain elusive. p38 mitogen activeted protein kinase (MAPK) signaling is one of the three major signal transduction pathways integrating and transmitting an extracellular stimulus as an intracellular signal. It has been reported that inhibition of this signaling pathway may protect against numerous and diverse disease processes, including models of arthritis, acute liver failure and cardiac hypertrophy and dysfunction. The aim of the present study was to define the role of p38 MAPK signaling in septic lung injury. Pulmonary edema, bronchoalveolar infiltration of leukocytes, levels of myeloperoxidase and CXC chemokines were determined 6 and 24 hours after cecal ligation and puncture (CLP). The specific p38 MAPK inhibitor SB 239063 was given immediately prior to CLP induction. Phosphorylation and activity of p38 MAPK were determined by immunoprecipitation and Western blot. CLP induced clear-cut pulmonary damage characterized by edema formation, leukocyte infiltration and increased levels of CXC chemokines in the lung. Moreover, CLP increased phosphorylation and activity of p38 MAPK in the lung, which was markedly inhibited by SB 239063. Interestingly, inhibition of p38 MAPK signaling protected against CLP-induced lung damage and edema. Indeed, SB 239063 dose-dependently decreased CLP-induced leukocyte recruitment in the bronchoalveolar space and formation of CXC chemokines in the lung. Our data demonstrate that p38 MAPK signaling constitutes a key role in regulating CXC chemokine production in septic lung injury and that inhibition of p38 MAPK activity abolishes pulmonary infiltration of leukocytes as well as lung edema. These novel findings demonstrate that p38 MAPK signaling pathway plays an important role in the recruitment of leukocytes in CLPinduced sepsis and thus targeting this signaling may pave the way for a new therapeutic strategy against lung injury in polymicrobial sepsis.

\section{O205}

\section{SIMVASTATIN PROTECTS AGAINST CHOLESTATIC LIVER INJURY} IN BILE DUCT LIGATED MICE

S.D. Dold ${ }^{1}$, L.W.M. Laschke ${ }^{2}$, D. Menger ${ }^{2}$, M.K.S. Schilling ${ }^{3}$,

B.J. Jeppsson ${ }^{4}$, H.T. Thorlacius ${ }^{4}$

1 University Of Saarland, Homburg/Saar, Germany

2 Clinical And Experimental Surgery, University Of Saarland, Homburg/

Saar, Germany

3 Clinic For General And Visceral Surgery University Of Saarland,

Homburg/Saar, Germany

4 Department Of Surgery, University Hospital Malmö, Sweden, Lund

University Malmö, Sweden

Background: Extrahepatic bile duct obstruction may cause severe liver damage and subsequent sepsis. Accumulating data suggest that statins, inhibitors of HMG-Co A reductase, may exert anti-inflammatory effects.

The aim of this study was to elucidate if statin may protect against cholestasis-induced liver injury

Methods: C57BL/6 mice underwent bile duct ligation (BDL) for 12 hours. Mice were pre-treated with vehicle, simvastatin $(0.2 \mathrm{mg} / \mathrm{kg})$ and simvastatin $(0.2 \mathrm{mg} / \mathrm{kg})$ combined with mevalonic acid $(10 \mathrm{mg} / \mathrm{kg})$, which is the main product of HMG-Co A reductase. Platelet and leukocyte recruitment as well as microvascular perfusion in the liver were determined by use of intravital fluorescence microscopy. Liver damage was monitored by measuring serum levels of alanine aminotransferase (ALT) and aspartate aminotransferase (AST). To quantify the extent of cholestasis serum concentration of total bilirubine was obtained.

Results: BDL caused a significant liver damage characterized by increased levels of liver enzymes and sinusoidal perfusion failure as well asmassive accumulation of leukocytes . Formation of widespread intravascular platelet aggregates were observed in postsinusoidal venules but mainly in liver sinusoids Simvastatin reduced levels of ALT and AST by more than $87 \%$ in BDL mice. Moreover, BDL-induced sinusoidal perfusion failure was reversed in simvastatin-treated animals. Simvastatin decreased leukocyte adhesion in both postsinusoidal venules than $73 \%$ in BDL mice. Also, BDL-induced formation of platelet aggregates was reduced by $77 \%$ in postsinusoidal venules and by $59 \%$ in sinusoids in simvastatin-treated animals. Interestingly, co-administration of mevalonic acid abolished all protecitve effects exerted by simavastatin in BDL mice. . Serum concentrations of bilirubin was similar in all experimental groups. Conclusion: Thus, our data demonstrates that simvastatin decrease both leukocyte and platelet accumulation in the liver microvasculature in cholestatic mice. These findings may explain the protective effect of simvastatin on cholestasis-induced liver damage. Moreover, our data suggest show that exogenous mevalonic acid reverse the benefical effects of simvastatin, suggesting that the protective effects of simvastatin are dependent on inhibition of HMG-Co A reductase. Taken together our findings suggest that simvastatin may be useful in protecting the liver in clinical conditions with cholestasis

\section{O206} INTENSIVE INSULIN THERAPY DURING CRITICAL ILLNESS
MODULATES NO METABOLISM SELECTIVELY VIA BLOOD GLUCOSE CONTROL

M.C. Richir ${ }^{1}$, B. Ellger ${ }^{2}$, L. Langouche ${ }^{2}$, Y. Debaveye ${ }^{2}$, I. Vanhorebeek ${ }^{2}$, P.A.M. van Leeuwen ${ }^{1}$, G. van den Berghe ${ }^{2}$

1 VU University Medical Center Amsterdam, Amsterdam,

The Netherlands

2 Catholic University Of Leuven, Leuven, Belgium

Introduction: Strict glycemic control by intensive insulin therapy (IIT) reduces morbidity and mortality of critically ill surgical patients. As potential mechanisms behind the clinical benefits emerge the prevention of overwhelming nitric oxide (NO) release and the regulation of local NO bio-availability. We hypothesized that glycemic control or glycemiaindependent effects of insulin modulate the transcriptional and posttranscriptional regulation of NOS-activity, possibly by affecting substrate availability of arginine for NO-synthases (NOS) or the plasma levels of the endogenous NOS-inhibitor asymmetric dimethylarginine (ADMA).

Methods: We assessed in an animal models of prolonged critical illness the relative impact of maintaining normoinsulinemia/normoglycemia, hyperinsulinemia/normoglycemia, normoinsulinemia/hyperglycemia, and hyperinsulinemia/hyperglycemia over 7 days on: NO plasma levels, NOS-activity, gene expression of endothelial (eNOS) and inducible (iNOS) isoforms of NOS in skeletal muscle, liver and aorta biopsies, and plasma levels of arginine and ADMA.

Results: Both hyperglycemic groups revealed increased plasma NO-levels, whereas NOS-activity in muscle and endothelium was decreased. Concomitantly, hyperglycemic groups revealed elevated gene-transcription of iNOS in muscle and eNOS in aorta. NO-levels, NOS-activity and NOS-mRNA were not different from healthy controls in both normoglycemic groups. Plasma arginine over ADMA ratio was low in hyperglycemic animals only. This ratio correlated with NOS-activity in the investigated tissues. Insulin exerted no glycemia-independent actions on any of the studied parameters.

Conclusion: In this animal model of prolonged critical illness, maintaining normoglycemia, and not glycemia-independent actions of insulin, prevented excessive NO release and preserved local NOS-activity, by modulating plasma levels of arginine and ADMA rather than by affecting NOS-gene transcription.

\section{O207}

CEA IN ACTIVATED MACROPHAGES: A NEW PROGNOSTIC OR DIAGNOSTIC FACTOR FOR EARLY DETECTION OF LOCAL RECURRENCE OF COLORECTAL NEOPLASMS?

\section{Japink' ${ }^{1}$, M.N. Sosef ${ }^{2}$, M.P.G. Leers², M. Nap ${ }^{2}$}

1 University Hospital Maastricht, Maastricht, The Netherlands

2 Atrium Medical Center Parkstad, Heerlen, The Netherlands

The Carcinoembryonic Antigen (CEA) is a protein that can be found in increased concentration in the blood of patients with colorectal cancer. It is used during follow-up for the detection of local tumor recurrence or metastases. 45 to 60 percent of these patients show neither an elevated CEA at the time of primary diagnosis, nor does it prove to be of much value during follow-up. Tumors demonstrate a continuous imbalance of cell proliferation and cell death, after which these cells or parts of these cells, enter the circulation. This principle should make it possible to find free tumor cells - or particles thereof - containing CEA, in the circulation. As macrophages phagocytize these tumor fragments, an analysis of CEA in activated macrophages could be a more sensitive parameter than CEA in serum. Detection of CEA in activated macrophages could 